\title{
Nonequilibrium dynamical mean-field theory
}

\author{
Dissertation im Fach Physik \\ vorgelegt der \\ Mathematisch-Naturwissenschaftlichen Fakultät \\ der Universität Augsburg
}

September 2009

angefertigt am

Lehrstuhl für Theoretische Physik III

- Elektronische Korrelationen und Magnetismus Institut für Physik der Universität Augsburg

bei Prof. Dr. D. Vollhardt

von

Martin ECKSTEIN 
Erstprüfer: Prof. Dr. D. Vollhardt

Zweitprüfer: Prof. Dr. Th. Kopp

Tag der mündlichen Prüfung: 21. Dezember 2009 


\section{Contents}

I Models and Methods 5

\begin{tabular}{lll}
\hline 1 & Models & 6
\end{tabular}

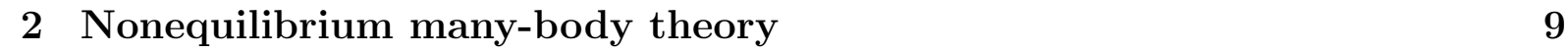

$2.1 \quad$ Definition of the nonequilibrium problem . . . . . . . . . . . . . . . . . . . 9

2.2 Keldysh contour Green functions . . . . . . . . . . . . . . . . . . . . . 10

2.2 .1 Historical remarks $\ldots \ldots \ldots$. . . . . . . . . . . . . 10

$2.2 .2 \quad$ Definition and basic properties . . . . . . . . . . . . . . . . 11

$2.2 .3 \quad$ Analytic properties . . . . . . . . . . . . . . . . . . . 14

2.3 Equations of motion and the self-energy $\ldots \ldots \ldots \ldots$. . . . . . . 17

2.4 Perturbation theory $\ldots \ldots \ldots \ldots \ldots \ldots$

2.5 Differential and integral equations on the contour . . . . . . . . . . 20

2.5 .1 The inverse of a contour function . . . . . . . . . . . . . . 22

2.5 .2 Volterra equations of the first and second kind . . . . . . . . . . 26

$\begin{array}{lll}3 & \text { Nonequilibrium dynamical mean-field theory } & 28\end{array}$

3.1 Dynamical mean-field theory . . . . . . . . . . . . . . . . . . . 28

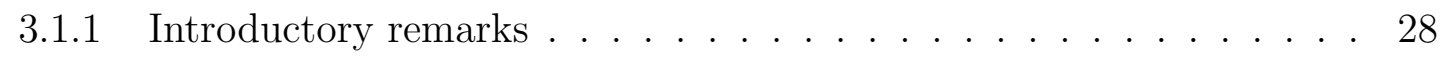

3.1 .2 DMFT for Keldysh-contour functions . . . . . . . . . . . . . . . . . 30

3.2 Nonequilibrium DMFT: the self-consistency condition . . . . . . . . . . . . 32

3.3 Calculation of observables $\ldots \ldots \ldots \ldots \ldots$

3.4 Nonequilibrium DMFT: Impurity solvers . . . . . . . . . . . . . . . . . . 38

3.4 .1 Introduction . . . . . . . . . . . . . . . . . . . . . . . 38

3.4 .2 Continuous-time Quantum Monte Carlo . . . . . . . . . . . . . . . 38

3.4 .3 Perturbative solution of the local problem . . . . . . . . . . . . . . . . . . . . . . 40

3.4.4 $\quad$ The mapping problem . . . . . . . . . . . . . . . . . . . . . . . . 42

II Time-evolution of simple model systems 49

$\begin{array}{|ll|}4 & \text { Approach to the thermal state }\end{array}$

4.1 Introduction . . . . . . . . . . . . . . . . . . . 50

4.2 Thermalization in classical and quantum systems . . . . . . . . . . . . . 51

4.3 Statistical predictions for integrable systems . . . . . . . . . . . . . . 56 
4.4 Prethermalization . . . . . . . . . . . . . . . . . . . . . . . . . . . . 62

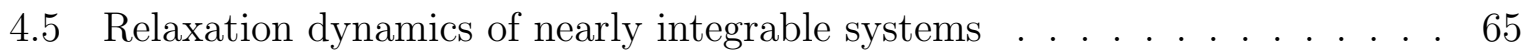

$\begin{array}{lll}5 & \text { Interaction quench in the Falicov-Kimball model } & \mathbf{7 0}\end{array}$

5.1 The Falicov-Kimball model . . . . . . . . . . . . . . . . . . . . . . 70

5.2 Interaction quench in the Falicov-Kimball model: Analytical solution . . . 73

$5.2 .1 \quad$ Equations of motion in nonequilibrium . . . . . . . . . . . . 73

$5.2 .2 \quad$ Langreth rules . . . . . . . . . . . . . . . . . . . . . . . 75

$5.2 .3 \quad$ Stationary states for $t, t^{\prime}<0$ and $t, t^{\prime} \rightarrow \infty \ldots \ldots . \ldots 76$

5.2 .4 Double Fourier transforms . . . . . . . . . . . . . . . . 78

5.2 .5 Back transformation . . . . . . . . . . . . . . . . . . . 80

5.3 Results: Non-thermal steady states . . . . . . . . . . . . . . . . . 81

5.3 .1 Time evolution of the double occupation . . . . . . . . . . . . . . . 81

5.3 .2 The steady state . . . . . . . . . . . . . . . . 83

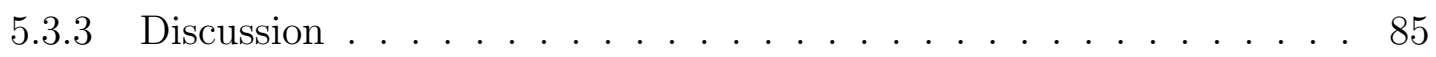

5.3 .4 Comparison to the strong-coupling expansion . . . . . . . . . . . . 89

\begin{tabular}{|lll}
6 & Interaction quench in the Hubbard model & 91
\end{tabular}

6.1 Preliminary remarks $\ldots \ldots \ldots \ldots \ldots$. . . . . . . . . . . . . . . . 91

6.2 Collapse of the Fermi-surface discontinuity . . . . . . . . . . . . . . . . . . 94

6.3 Relaxation after the quench . . . . . . . . . . . . . . . . . . . . 96

7 Slow changes of the Hamiltonian: The crossover to adiabatic behavior 100

7.1 Introduction . . . . . . . . . . . . . . . . . . 100

7.2 Falicov-Kimball model: DMFT . . . . . . . . . . . . . . . . . 102

7.2 .1 Preliminary remarks . . . . . . . . . . . . . . . . 102

7.2 .2 Results . . . . . . . . . . . . . . . . . . . . . . . . 104

7.3 Perturbation theory for small ramps . . . . . . . . . . . . . . 105

7.3 .1 Derivation . . . . . . . . . . . . . . . . . . . 105

$7.3 .2 \quad$ Insulating phase . . . . . . . . . . . . . . . . . . . . 108

7.3 .3 Metallic phase . . . . . . . . . . . . . . . . . . . 110

III Time-resolved spectroscopy on solid-state systems $\quad 115$

8 Time-resolved spectroscopy on correlated systems $\quad 116$

8.1 Pump-probe experiments . . . . . . . . . . . . . . . . 116

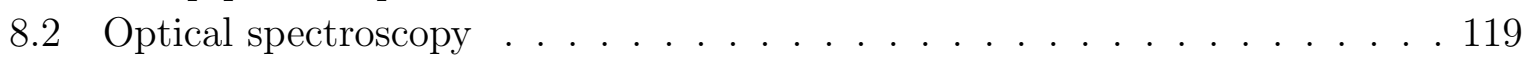

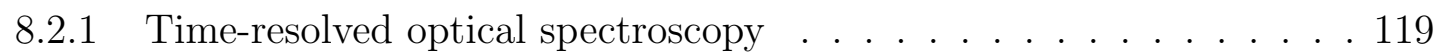

8.2 .2 Optical Conductivity in DMFT . . . . . . . . . . . . . . . . . . 121

8.2 .3 The sum rule for the two-time optical conductivity . . . . . . . . . 124

8.3 Photoemission spectroscopy in the sudden approximation . . . . . . . . . . . 124

8.3 .1 Introduction . . . . . . . . . . . . . . . . . . . 124

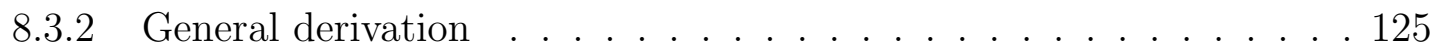


8.3.3 Role of the energy-time uncertainty relation . . . . . . . . . . . . . 127

8.4 Evaluation within DMFT $\ldots \ldots \ldots \ldots$

8.4 .1 Buildup of correlations in the metallic phase . . . . . . . . . . . . . 131

8.4 .2 Coherent electronic oscillations in time-resolved spectra . . . . . . . . 134

$\begin{array}{lll}9 & \text { Conclusion and outlook } & 139\end{array}$

\begin{tabular}{|l} 
A Combination of CTQMC and DMFT \\
\hline
\end{tabular} 



\section{Introduction}

The electrostatic interaction between the electrons a solid and between electrons and the lattice ions leads to complex material properties, such as metal-insulator transitions, hightemperature superconductivity, and various magnetic phenomena. When such interacting many-particle systems are driven out of equilibrium, a multitude of yet unexplored phenomena can be found. Their understanding is often related to questions that lie at the basis of statistical physics. On the other hand, the highly nonlinear material properties promise that those systems will once find an application in special devices, e.g., optical switches which operate much faster than conventional electronic ones. The investigation of such interacting many-particle systems out of equilibrium is the main focus of this thesis.

It is worthwile to note that most experiments in condensed matter physics are performed in a regime where one can safely assume that the systems are in equilibrium on the atomic length scale, or that they are only slightly perturbed from their equilibrium state. In contrast, the preparation and investigation of nonequilibrium states requires rather extreme experimental conditions. Because the typical energy scale for the valence electrons in a solid is of the order of $\mathrm{eV}$, the corresponding timescale $\hbar / \mathrm{eV} \approx 1 \mathrm{fs}\left(1 \mathrm{fs}=10^{-15}\right.$ seconds) is extremely short, and the relevant electrical field of the order of $1 \mathrm{~V}$ per few lattice spacings is extremely large compared to what can usually be reached in experiment. In other words, very strong fields are needed to drive such a system ofut of equilibrium, and it will quickly relax back to an equilibrium state when it is left alone after the perturbation. Nevertheless, several experimental techniques have been advanced in recent years which allow to investigate interacting quantum many-particle systems out of equilibrium. Firstly, the dynamics of the valence electrons and the lattice can be observed through time-resolved spectroscopy with ultrashort laser pulses. In those experiments, an intense laser pulse drives the system out of equilibrium, and a delayed pulse is used to take a snapshot of the time-evolving state. The delay between the two pulses is easily controllable by a modification of the optical path, such that the main technological challenge consists in the generation of short laser pulses. Those pulses are now becoming available even on the attosecond range, and free-electron lasers can generate femtosecond pulses in the soft x-ray regime to probe the lattice dynamics in real time. Apart from pump-probe experiments, nonequilibrium physics plays a mayor role for the understanding of transport through molecules or quantum dots, where electrical fields can become very large in the region of interest. Furthermore, the time evolution of interacting many-body systems can be investigated using ultracold atomic gases in optical lattices. Those systems are very 
well isolated from environment and can be described in terms of simple model Hamiltonians. The Hamiltonian parameters and their variation with time is easily controllable in experiment, and because the relevant energies are orders of magnitude smaller than in solid state systems the quantum dynamics takes place on the timescale of milliseconds which can almost be resolved with the human eye.

The dynamics of a correlated system reveals many effects that are more than just a featureless relaxation to equilibrium. For example, some nonlinear phenomena occur only above a certain excitation threshold, or dynamical phase transitions may be found, at which an abrupt change in the relaxation behavior occurs upon small changes of the Hamiltonian parameters and the excitation energy. Furthermore, the dynamics of correlated systems is related to questions that touch the foundations of statistical physics. Under what conditions can a system relax to thermal equilibrium at all? Is this only possible due to dissipation to the environment? Or is thermalization and ergodic behavior a generic property of the complex but nevertheless reversible dynamics of interacting many-particle systems, in the sense that the memory of the initial state can be recovered only from extremely complicated measurements which are impossible in practice, while thermodynamic observables show averaged behavior in the long term? Using ultracold atomic gases it has already been demonstrated that a collection of many interacting particles can behave nonergodic on rather long timescales, such that a detailed memory on the initial state remains imprinted in simple observables and correlation functions of the system. This behavior has been related to integrability, but the transition from the nonergodic behavior of integrable system to the presumably ergodic behavior of nonintegrable models is still not resolved in quantum mechanics.

The understanding of those phenomena and questions is a challenge for theoretical physics, and completely new theoretical tools have to be developed to guide the experimental progress. Those methods must cope with both nonequilibrium phenomena and strong correlations in a nonperturbative way. Furthermore they should be applicable to large systems, because the time evolution in small systems is dominated by irregular oscillations and recurrences. The generalization of dynamical mean-field theory (DMFT) to nonequilibrium phenomena combines all these properties. In equilibrium physics, the method has already proven to give a good description of correlated systems at least in dimension $d>2$, and it becomes exact in the limit of infinite dimensions. DMFT is now routinely applied to calculate properties of real materials, and extensions are developed for inhomogeneous and low-dimensional systems. Recently, it has been noted that DMFT can be extended to nonequilibrium situations by using the Keldysh formalism, and it is the purpose of this thesis to continue this development. It will be shown that nonequilibrium DMFT indeed does predict interesting nonequilibrium phenomena in the Falicov-Kimball model and the Hubbard model, which may be observed in experiment. Furthermore, the theoretical description of time-resolved spectroscopy on solid-state systems within DMFT is clarified. Whithin this thesis, the investigation of pump-probe experiments on correlated systems is still based on idealized model situations. While this does not yet allow for a direct comparison to experiment, such an analysis lays the foundations to start more realistic calculations in the near future. In will then become clear whether DMFT will 
once play a comparable role in nonequilibrium physics as it does today in equilibrium physics.

\section{Outline of this thesis}

In Ch. 1 we introduce the Hubbard model and the notion of the quantum quench, which is the basic setup for most problems that are discussed in the remainder. The mathematical framework for nonequilibrium DMFT is introduced in Ch. 2, using the language of the Kadanoff-Baym equations which are adapted to allow for an efficient description of the initial states in terms of its equilibrium spectral function. In Ch. 3, nonequilibrium DMFT is explained, together with some progress in the development of the method which was achieved within this thesis. For example, we derive the mapping of the DMFT equations to an impurity Hamiltonian in nonequilibrium, which is the prerequisite to use timedependent density matrix renormalization group (t-DMRG) or other powerful numerical techniques, which are currently developed to describe transport in quantum dots, in the context of DMFT.

Ch. 4 introduces the fundamental question of thermalization in quantum systems. This chapter uses only basic quantum mechanics and statistical physics, and is thus independent of DMFT. In particular, we discuss the description of nonthermal steady states in integrable and nonintegrable systems. In some cases, such states can be described in terms of a statistical prediction, and general criteria are derived when this is possible (Sec. 4.3). The following chapters are then devoted to specific models which are solved within nonequilibrium DMFT: In Ch. 5 we derive the exact solution of the Falicov-Kimball model after a quantum quench. On the one hand, this serves as an important benchmark for numerical approaches, while on the other hand, the model provides an example of a system which does not thermalize. A similar analysis as for the Falicov-Kimball model is then repeated for the Hubbard model (Ch. 6). In this case, DMFT can only be solved numerically, for which purpose we use the real-time continuous-time Monte Carlo technique that has recently been developed by Ph. Werner and coworkers. The physics of the Hubbard model turns out to be completely different from that of the Falicov-Kimball model. In the weak- and strong-coupling regime, the system is trapped in nonthermal quasisteady states on long timescales, while in an intermediate interaction range rapid thermalization is observed. These results indicate the existence of a dynamical phase transition in the Hubbard model. Finally, Ch. 7 focuses at a different kind of nonequilibrium problem, namely the excitation of a system during a slow transition between two parameters. The last part of this thesis (Ch. 8) explains how the experimental signals of pump-probe spectroscopy in solids can be described with nonequilibrium DMFT. 

Part I

Models and Methods 


\section{Chapter 1}

\section{Models}

A solid is a collection of a macroscopic number of interacting nuclei and electrons. The microscopic Hamiltonian that includes all those degrees of freedom on an equal footing is easily written down, but without additional input from intuition and experiment it is of little practical use for the understanding of the physical properties of solids. The introduction of effective models that contain fewer degrees of freedom and simplified interactions is thus an essential step in the solution of the full microscopic theory of solids. The "derivation" of those models, which can hardly be performed with mathematical rigor, proceeds via the separation of time- and energy scales. Furthermore, in the typical behavior of a real solid many intersting phenomena can be observed at the same time. Minimal models are thus not only an intermediate step towards a full solution of the microscopic theory, but they are the key instrument for the microscopic description and classification of the various phases that are observed in solids.

Throughout this thesis special attention will be paid to the Hubbard model and related models. The Hubbard Hamiltonian was first independently introduced by Hubbard [1], Gutzwiller [2], and Kanamori [3] in 1963 in order to explain itinerant ferromagnetism. The model describes electrons on a crystal lattice which interact through a local Coulomb repulsion. The Hamiltonian is given by

$$
\begin{aligned}
& H=H_{p o t}+H_{k i n} \\
& H_{p o t}=\sum_{i}\left(n_{i \uparrow}-\frac{1}{2}\right)\left(n_{i \downarrow}-\frac{1}{2}\right) \\
& H_{k i n}=\sum_{i j \sigma} V_{i j \sigma} c_{i \sigma}^{\dagger} c_{j \sigma} .
\end{aligned}
$$

Here $c_{i \sigma}^{\dagger}\left(c_{i \sigma}\right)$ are creation (annihilation) operators for an electron with spin $\sigma$ in a valence orbital at site $i$, and $n_{i \sigma}=c_{i \sigma}^{\dagger} c_{i \sigma}$ is the local density. The potential energy is due to on-site Coulomb interaction between the electrons with interaction strength $U$, and the kinetic energy $H_{k i n}$ describes the hopping of electrons on the lattice, with amplitudes $V_{i j \sigma} \equiv V t_{i j \sigma}$. ( $V$ denotes the energy scale, and $t_{i j \sigma}$ the relative strength of the matrix elements.) Spin-dependent hopping is allowed to cover a simplified variant of the Hubbard model where one spin-species is immobile (the Falicov-Kimball model, Ch. 5). 
In spite of its apparent simplicity, a solution of the Hubbard model has been found only in one dimension [4]. However, various approximate methods and exactly solvable limits exist, such as perturbation expansions around the limits of vanishing interaction (a band metal) and vanishing hopping (the so-called atomic limit), and the limit of infinite dimensions (Ch. 3). Alltogether those results prove a fairly rich equilibrium phase diagram of the Hubbard model. For half-filling and bipartite lattices the low-temperature phase is antiferromagnetically ordered. This result is obtained both in weak-coupling and strongcoupling theory. In the strong-coupling limit the Hubbard model can be mapped onto an effective Heisenberg model [5]. Ferromagnetism is rigorously established for one electron less than half-filling and $U \rightarrow \infty$ by Nagaoka [6]. Of particular interest for this thesis is the paramagnetic phase, which undergoes a metal-insulator transition at a critical interacting strength $U>0$ in dimension $d>1$. This Mott transition, at which the electrons become localized due to the on-site Coulomb repulsion is observed in many real materials [7, 8]. In the Hubbard model it was first indicated by early approximations of Hubbard himself [9] and an application of the Gutzwiller variational wave function [10], and later intensively studied within dynamical mean-field theory [11].

Compared to the physics of real materials the Hubbard model entails several simplifications of which we now mention two which are particularly important. (i) The nuclei of the crystal lattice are considered as immobile, and electron-phonon interaction is thus completely neglected. This is the lowest order of the Born Oppenheimer approximation, where the nuclear motion is considered as slow compared to the motion of the electrons, such that the electrons are always in an instantaneous equilibrium state corresponding to fixed atomic positions. (ii) Only one band is taken into account in the Hubbard model. The excitation to higher (empty) bands is assumed to be so large that those excitations do not play any role, while the core electrons are tightly bound to the nuclei. The polarization of the resulting charged ions contributes to the screening of the long-range Coulomb interaction between the valence electrons to the on-site repulsion of the Hubbard model, but is disregarded otherwise. There are several generalizations of the Hubbard model to more realistic model which can then compared to real materials, but their discussion is beyond the scope of this thesis. However, the use of ultracold gases in optical lattices [12] has recently allowed to realize various idealized models of solid state physics experimentally, including the single-band Hubbard model. In these experiments both the interaction and the hopping of the atoms can be tuned in a wide range, and the system is in perfect isolation from environment. The Mott-transition has been observed in optical lattices for the bosonic Hubbard model [13] and very recently also for the fermionic model (1.1) [14, 15].

In this thesis we will discuss the time evolution of the Hubbard model and related models. The simplest problem that can be studied in this context is a quantum quench, where the system is prepared in a thermal equilibrium state with grand-canonical density matrix $\rho=e^{-\beta(H-\mu N)} / Z$ or in the ground state of the Hamiltonian at time $t<0$, and at $t=0$ some parameter, e.g., the interaction $U$, is suddenly changed to a new value, and from thereon the system evolves freely for $t>0$. This setup is frequently studied in the literature for various models (Ch. 4). It allows to investigate the relaxation of the many- 
body system in a highly idealized way. In experiments with cold atoms, such a quantum quench can be realized [16] due perfect isolation from environment and controllability of the parameters. On the other hand, the observation of the real-time evolution of interacting many-body systems is not restricted to those highly idealized situations. In solid state systems, nonequilibrium states can be induced and probed using time-resolved spectroscopy. This is discussed in Ch. 8. In those experiments, it is nontrivial to determine whether a specific situation can be described in terms of a simple Hamiltonian such as Eq. (1.1). Although, e.g., the coupling to the lattice and the motion of the nuclei may be disregarded for the description of equilibrium properties, it can - and often does - play and important role for the nonequilibrium states. However, in Ch. 8 we will show that in some situations the electrons can be considered as isolated from the rest of the lattice on short time-scales, and thus some aspects of the time evolution of these systems may be qualitatively described by the purely electronic Hubbard model.

For the description of those spectroscopic experiments, external electromagnetic fields have to be incorporated into the Hamiltonian (1.1). The inclusion of electromagnetic fields in effective lattice models is a rather complicated topic. Starting from the microscopic description, one has to understand how neglected bands influence the propagation of electromagnetic waves in the solid, and how the presence of electromagnetic fields effects both the interaction and the hopping terms in the effective model for the valence electrons. Here we will only state the common way to include electromagnetic fields into the Hubbard model [17, 18] without going into the details of the microscopic justifications. In the presence of electromagnetic fields [with scalar and vector potential $\Phi(\boldsymbol{r}, t)$ and $\boldsymbol{A}(\boldsymbol{r}, t)$ ], the hopping amplitudes $V_{i j \sigma}$ in (1.1) acquire Peierls phase factors [19]

$$
V_{i j \sigma}=\widetilde{V}_{i j \sigma} \exp \left(\frac{i e}{\hbar c} \int_{\boldsymbol{R}_{i}}^{\boldsymbol{R}_{j}} d \boldsymbol{r} \boldsymbol{A}(\boldsymbol{r}, t)\right),
$$

and a potential term $-e \sum_{i \sigma} \Phi\left(\boldsymbol{R}_{i}, t\right) c_{i \sigma}^{\dagger} c_{i \sigma}$ is added to the Hamiltonian. Here $-e$ is the charge of an electron, the tilde indicates that the quantity is taken in zero external field, and the integral goes along a straight line between the lattice sites. The electromagnetic field is then determined by the Maxwell equations, where the current is given by the expectation value of $\boldsymbol{j}(\boldsymbol{r})=-c \delta H / \delta \boldsymbol{A}(\boldsymbol{r})$ (cf. Ch. 8) (and an additional dielectric constant can account for polarization of core electrons and lattice.) The Peierls factors can be derived for well localized Wannier orbitals [20, 21]. It should be noted, however, that a straightforward generalization of $(1.2)$ is difficult for multiband systems (see, e.g., Ref. [22]). The latter would be needed to describe strong-field phenomena, such as the Zener breakdown of band insulators, which is a tunneling process between filled and empty bands. 


\section{Chapter 2}

\section{Nonequilibrium many-body theory}

\subsection{Definition of the nonequilibrium problem}

Although the field of nonequilibrium phenomena is in principle much broader than equilibrium thermodynamics, it is by far less studied. The reason for this is the remarkable universality of equilibrium systems, whose properties depend only on very few parameters. In contrast, the characterization of a nonequilibrium state usually requires detailed knowledge about its preparation. The purpose of this chapter is to give a clear mathematical definition of the nonequilibrium problems that will be discussed in the remainder of this thesis, and introduce the theoretical tools that are needed to study them from a microscopic point of view.

Problems in nonequilibrium physics may be classified according to (i), whether they deal with open or closed systems, and (ii), whether the focus is on the transient time evolution during and after some perturbation, or the stationary state that possibly develops when a system is driven by external fields. Open systems are coupled to a dissipative environment, while the dynamics of a closed system is completely described in terms of its time-dependent Hamiltonian $H(t)$. The latter may include external fields that act on the system, but no coupling to a heat bath. This thesis deals almost entirely with the transient time evolution of closed quantum systems, including the approach of a stationary state within this transient time evolution. A general experiment in this context is described by the solution $|\psi(t)\rangle$ of the Schrödinger equation,

$$
i \frac{d}{d t}|\psi(t)\rangle=H(t)|\psi(t)\rangle
$$

with a "prepared" initial state $\left|\psi\left(t_{\text {min }}\right)\right\rangle=\left|\psi_{0}\right\rangle$ at some early time $t_{\text {min }}$. from $|\psi(t)\rangle$ the expectation values $O(t)=\langle\psi(t)|O| \psi(t)\rangle$ of observables $O$ (Hermitian operators) at later times $t>t_{\min }$ can be obtained. We set $\hbar=1$, i.e., time is measured in units of $1 /$ energy.

The time evolution of a closed system is unitary, $|\psi(t)\rangle=\mathcal{U}\left(t, t_{\min }\right)\left|\psi\left(t_{\min }\right)\right\rangle$, and the time evolution operator $\mathcal{U}\left(t, t_{\text {min }}\right)$ is determined by the differential equation

$$
i \partial_{t} \mathcal{U}\left(t, t^{\prime}\right)=H(t) \mathcal{U}\left(t, t^{\prime}\right), \quad \mathcal{U}(t, t)=1
$$


which can be integrated to

$$
\mathcal{U}\left(t, t^{\prime}\right)=\left\{\begin{array}{l}
T_{t} \exp \left[-i \int_{t^{\prime}}^{t} d \bar{t} H(\bar{t})\right] \quad t>t^{\prime} \\
\bar{T}_{t} \exp \left[-i \int_{t^{\prime}}^{t} d \bar{t} H(\bar{t})\right] \quad t<t^{\prime}
\end{array},\right.
$$

where $T_{t}\left(\bar{T}_{t}\right)$ is the (anti) time-ordering operator. Using this notation the basic problem of nonequilibrium of calculating observables and their time-dependent correlation functions is to obtain expectation values

$$
\langle\hat{O}(t)\rangle_{0},\left\langle\hat{O}_{1}\left(t_{1}\right) \hat{O}_{2}\left(t_{2}\right)\right\rangle_{0}, \ldots,
$$

where operators with a hat are in the Heisenberg picture,

$$
\hat{O}(t)=\mathcal{U}\left(t_{\min }, t\right) O \mathcal{U}\left(t, t_{\min }\right),
$$

and the initial state is generalized from a pure statae $\left|\psi_{0}\right\rangle$ to an average over initial states by means of an initial state density matrix $\rho_{0}$,

$$
\langle\cdot\rangle_{0}=\operatorname{Tr}\left[\rho_{0} \cdot\right], \quad \rho_{0}=\sum_{\nu} p_{\nu}\left|\psi_{\nu}\right\rangle\left\langle\psi_{\nu}\right|
$$

Averaging over initial states represents our insufficient knowledge of the true initial state, but does not imply any stochastic elements in the time evolution. Even when we choose the mixed initial state $\rho_{0}$ as the grand-canonical density matrix of the Hamiltonian at time $t=t_{\min }$ (which will often be the case in the following) this does not mean that the system is coupled to a heat or particle reservoir for $t>t_{\min }$. The grand-canonical density matrix is appropriate when the initial state is prepared by letting the system thermalize with heat and particle baths at fixed temperature and chemical potential, but for $t>$ $t_{\text {min }}$ the system must be decoupled from all reservoirs. In practice, this means that the coupling to the environment must be so small that it can be neglected on the timescale of the experiment.

The calculation of the correlation functions $(2.4)$ is the basic goal for any microscopic nonequilibrium theory. The mathematical treatment of those problems relies on the use of real-time Green functions which are introduced in the following section. Many introductory text are available in this field, e.g., Refs. [23, 24]. Nevertheless, in order to fix the notation and give a self-contained presentation, also the discussion of some basic concepts is included in the present chapter.

\subsection{Keldysh contour Green functions}

\subsubsection{Historical remarks}

Standard many-body techniques were first extended to the nonequilibrium case by Kadanoff and Baym [25], who introduced a set of equations of motion for two real-time Green functions $\left(G^{>}\right.$and $\left.G^{<}\right)$and from them derived systematic approximations such as a perturbation series in the interaction. These Kadanoff-Baym (KB) equations are now a standard 
way to obtain nonequilibrium transport equations, in particular, the quantum Boltzmann equation. This was already a main motivation for the original work (Ref. [25], Chapter 11; for reviews, consider Refs. [26, 27]). After the work of Kadanoff and Baym, Keldysh [28] introduced a diagrammatic perturbation theory, for which he generalized the time arguments of real-time Green functions to a contour that runs back and forth in time. The closed contour ensures that Wicks theorem, which is the basis for an efficient evaluation of the perturbation series, can be applied in the nonequilibrium case. The same contour had been used before by Schwinger in a different context [29].

Both the approaches by Kadanoff and Baym and by Keldysh have the disadvantage that they essentially assume the system to be in a noninteracting state at time $t=-\infty$, and thus neglect correlations in the initial state. Some proposals have been made to overcome this problem and explicitly include initial correlations [30, 31], and to prove under which conditions initial correlations in the KB equations can be neglected [32]. The problem was finally solved in an elegant form by Danielewicz [33] by extending the original Keldysh time-contour to a different one that involves time propagation along imaginary time. This L-shaped contour, which was later rediscovered (see, e.g., Ref. [34]), is appropriate for situations where the system is initially in thermal equilibrium. It allows to formulate perturbation theory for Matsubara and nonequilibrium Green functions in an equivalent language, and presents a straightforward way to derive KB equations which include initial correlations [35] (cf. Sec. 2.3). The L-shaped contour $\mathcal{C}$ provides the basis of many nonequilibrium Green function calculations, e.g., the perturbative description of weakly correlated semiconductors or mesoscopic systems in nonequilibrium [36].

\subsubsection{Definition and basic properties}

In this section we will introduce contour Green functions on the contour that is adapted to the description of the transient time evolution of a system which is prepared in a thermal equilibrium state at initial time $t=t_{\min }$. In the following we assume that our system has a Fock space 1 with single-particle basis $\{|\alpha\rangle\}$ (e.g., momentum, space, spin), and let $c_{\alpha}\left(c_{\alpha}^{\dagger}\right)$ denote the annihilation (creation) operators for a particle in state $|\alpha\rangle$. Throughout this text we will consider only fermionic particles, so that the usual anticommutation relations $\left\{c_{\alpha}, c_{\alpha^{\prime}}^{\dagger}\right\}=\delta_{\alpha \alpha^{\prime}}$ and $\left\{c_{\alpha}^{\dagger}, c_{\alpha^{\prime}}^{\dagger}\right\}=\left\{c_{\alpha}, c_{\alpha^{\prime}}\right\}=0$ hold. The contour-ordered single-particle Green function for a general action $\mathcal{S}$ is then defined as

$$
G_{\alpha \alpha^{\prime}}\left(t, t^{\prime}\right)=-i \frac{\operatorname{Tr}\left[\mathrm{T}_{\mathcal{C}} \exp (\mathcal{S}) c_{\alpha}(t) c_{\alpha^{\prime}}^{\dagger}\left(t^{\prime}\right)\right]}{\operatorname{Tr}\left[\mathrm{T}_{\mathcal{C}} \exp (\mathcal{S})\right]} .
$$

This expression needs some further explanations:

(i) The time arguments $t$ and $t^{\prime}$ lie on the L-shaped contour $\mathcal{C}$ that runs from $t_{\min }$ to some large time $t_{\max }$ (i.e., the largest time of interest) on the real time axis, back to $t_{\min }$, and finally to $t_{\min }-i \beta$ on the imaginary time axis (Fig. 2.1). The contourordering $\mathrm{T}_{\mathcal{C}}$ exchanges the order of two operators $A\left(t_{1}\right)$ and $B\left(t_{2}\right)$ in a product

\footnotetext{
${ }^{1}$ The basic concepts of many-particle physics are explained, e.g., in Ref. 37.
} 


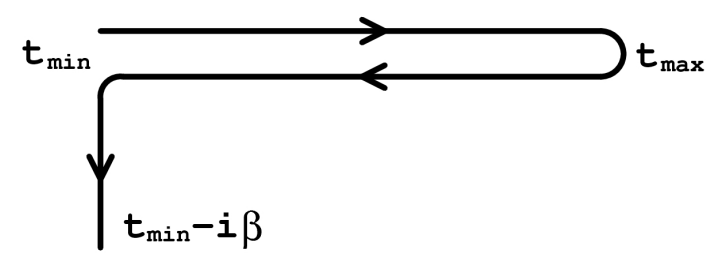

Figure 2.1: The L-shaped contour $\mathcal{C}$ for transient-nonequilibrium problems with thermal state at time $t=t_{\min }$.

$A\left(t_{1}\right) B\left(t_{2}\right)$ if and only if $t_{2}$ appears later on the contour than $t_{1}$ (we denote this ordering by $t_{2} \succ t_{1}$ ),

$$
\mathrm{T}_{\mathcal{C}} A\left(t_{1}\right) B\left(t_{2}\right)=\left\{\begin{array}{rr}
A\left(t_{1}\right) B\left(t_{2}\right) & t_{1} \succ t_{2} \\
\pm B\left(t_{2}\right) A\left(t_{1}\right) & t_{1} \prec t_{2}
\end{array} .\right.
$$

The minus sign in the second line applies to the case when both $A$ and $B$ are Fermi operators. The order of time arguments along $\mathcal{C}$ is indicated by the arrows in Fig. 2.1, which point from "earlier" to "later" times. Operators (without hat) are in the Schrödinger picture, i.e., their time argument only specifies how they are to be ordered along the contour.

(ii) For the action $\mathcal{S}$ we will consider two terms,

$$
\begin{aligned}
\mathcal{S} & =\mathcal{S}_{1}+\mathcal{S}_{2} \\
\mathcal{S}_{1} & =-i \int_{\mathcal{C}} d \bar{t} H(\bar{t}) \\
\mathcal{S}_{2} & =-i \sum_{\alpha_{1} \alpha_{2}} \int_{\mathcal{C}} d t_{1} \int_{\mathcal{C}} d t_{2} c_{\alpha_{1}}^{\dagger}\left(t_{1}\right) \Lambda_{\alpha_{1} \alpha_{2}}\left(t_{1}, t_{2}\right) c_{\alpha_{2}}\left(t_{2}\right),
\end{aligned}
$$

where $\int_{\mathcal{C}}$ denotes the integral along $\mathcal{C}$ (see Table 2.1). Eq. 2.7) is then understood such that $\exp (\mathcal{S})$ is expanded in powers of $\mathcal{S}_{2}$, and $\mathrm{T}_{\mathcal{C}}$ acts on each term separately. The first part $\mathcal{S}_{1}$ of the action accounts for the time evolution due to the Hamiltonian $H(t)$. The latter depends only on physical time, i.e., it is the same on the upper and lower horizontal part of the contour and constant on the imaginary branch, $H\left(t_{\min }-i \tau\right)=H\left(t_{\min }\right)$. If $\Lambda=0$ in $(2.9 \mathrm{c})$, the Green function (2.7) reduces to

$$
\left.G_{\alpha \alpha^{\prime}}\left(t, t^{\prime}\right)=-i\left\langle\mathrm{~T}_{\mathcal{C}} \hat{c}_{\alpha}(t) \hat{c}_{\alpha^{\prime}}^{\dagger}\left(t^{\prime}\right)\right]\right\rangle_{0}
$$

where $\hat{c}$ are Heisenberg operators, and the expectation value $\langle\cdot\rangle_{0}=\operatorname{Tr}\left[e^{-\beta H\left(t_{\min }\right)} \cdot\right]$ $/ \operatorname{Tr}\left[e^{-\beta H\left(t_{\min }\right)}\right]$ is taken in the initial equilibrium state. One can directly check this by writing out various parts of the integral in the exponent explicitly: The integral along the vertical part yields the initial state density matrix $e^{-\beta H\left(t_{\min }\right)}$ because $H(t)$ is constant along the imaginary branch, and the denominator gives $\operatorname{Tr}\left[\mathrm{T}_{\mathcal{C}} \exp \left(\mathcal{S}_{1}\right)\right]=$ $\operatorname{Tr}\left[e^{-\beta H\left(t_{\text {min }}\right)}\right]$ because forward and backward time evolution on the real branch (the integrals along upper and lower real-time branch) cancel each other. The second 
part of the action, $\mathcal{S}_{2}$, is non-local on the contour, and we assume that it contains no singular (local) terms. Although only unitary time evolutions are considered in this text, a nonlocal term occurs automatically when effective dynamics of only some degrees of freedom is considered, such as in DMFT (Ch. 3).

(iii) The Green function 2.7) for fermions satisfies an antiperiodic boundary condition in both time arguments

$$
\begin{aligned}
& G_{\alpha \alpha^{\prime}}\left(t_{\min }^{+}, t\right)=-G_{\alpha \alpha^{\prime}}\left(t_{\min }-i \beta, t\right) \\
& G_{\alpha \alpha^{\prime}}\left(t, t_{\min }^{+}\right)=-G_{\alpha \alpha^{\prime}}\left(t, t_{\min }-i \beta\right) .
\end{aligned}
$$

$\left(t_{\min }^{+}\right.$is the point $t=t_{\min }$ on the upper branch of $\mathcal{C}$.) This follows directly from the cyclic property of the trace and the definition of the contour ordering. Later we will introduce contour functions which have no simple definition in terms of annihilation and creation operators (e.g., the self-energy), but they will always satisfy this boundary condition.

(iv) If not stated otherwise, the contour-functions $A\left(t, t^{\prime}\right)$ considered in the following satisfy the condition that

$A\left(t, t^{\prime}\right)$ is unchanged when real-time arguments are shifted from the upper to the lower horizontal part of the contour such that their relative order is not affected.

For example, when $t$ is on the imaginary branch and $t^{\prime}$ is real, it does not matter whether $t^{\prime}$ is on the upper or lower real branch. In particular, 2.12 holds for the Green function (2.7) when it holds for $\Lambda$, because $H(t)$ depends only on physical time.

In the following we will use the following shorthand notation

$$
\langle A\rangle_{\mathcal{S}}=\frac{\operatorname{Tr}\left[\mathrm{T}_{\mathcal{C}} \exp (\mathcal{S}) A\right]}{\operatorname{Tr}\left[\mathrm{T}_{\mathcal{C}} \exp (\mathcal{S})\right]}
$$

Many-particle Green functions are defined in the same way as 2.7), e.g.

$$
G\left(1, \ldots, n ; n^{\prime} \ldots 1^{\prime}\right)=(-i)^{n}\left\langle c(1) \ldots c(n) c^{\dagger}\left(n^{\prime}\right) \ldots c^{\dagger}\left(1^{\prime}\right)\right\rangle_{\mathcal{S}},
$$

where the condensed notation $j=\left(t_{j}, \alpha_{j}\right)$ for contour time arguments and single-particle indices is used.

\section{Real-time-components}

In working with contour Green functions it is crucial to choose the right parameterization, and the straightforward notation which is used in Table 2.1) is often not the best way to do this. Various real-time, imaginary-time, and mixed components of the contour Green function, which are commonly used in the literature, are defined in Table 2.2. They are 


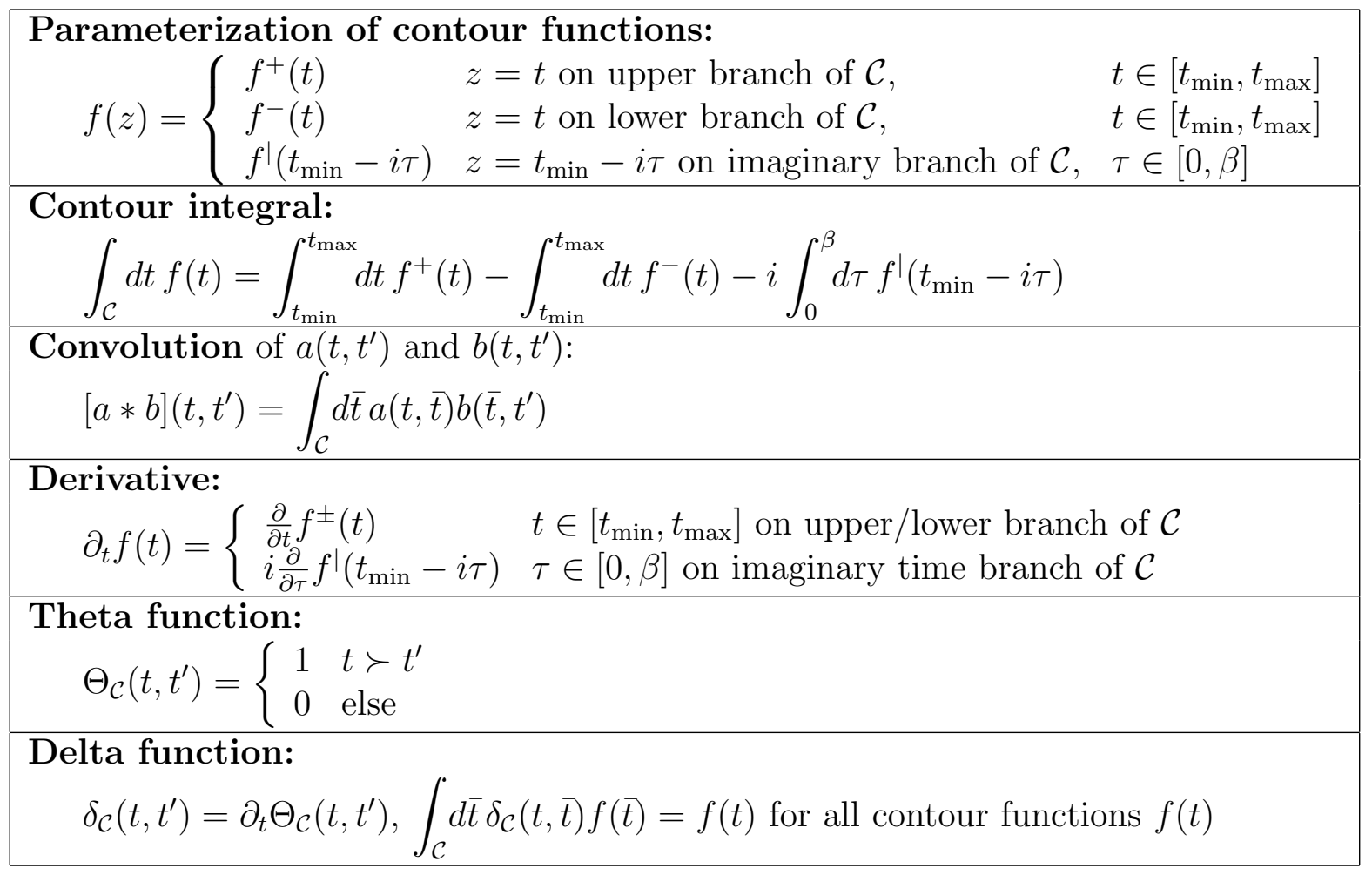

Table 2.1: Notation for contour-calculus used in this text.

not independent due to condition 2.12), and corresponding relations are also indicated in Table 2.2. In addition to those general relations, we frequently have the symmetry between the components

$$
\begin{aligned}
& G_{\alpha \alpha^{\prime}}^{\mathrm{r}}\left(t, t^{\prime}\right)=G_{\alpha^{\prime} \alpha}^{\mathrm{a}}\left(t^{\prime}, t\right)^{*} \\
& G_{\alpha \alpha^{\prime}}^{<}\left(t, t^{\prime}\right)=-G_{\alpha^{\prime} \alpha}^{<}\left(t, t^{\prime}\right)^{*} \\
& G_{\alpha \alpha^{\prime}}^{\neg}(t, \tau)=G_{\alpha^{\prime} \alpha}^{-}(\beta-\tau, t)^{*} .
\end{aligned}
$$

In particular, these relations follow for (2.7) when they hold for $\Lambda$ in (2.9c).

\subsubsection{Analytic properties}

Equilibrium Green functions have a number of useful analytic properties. In this section we briefly review these properties and derive similar properties of the nonequilibrium functions. This will be done only for the Green function 2.10 , i.e., when the non-local term $(2.9 \mathrm{c})$ vanishes in the action $(2.9)$. The generalization to $\Lambda \neq 0$ will become clear from Sec. 2.5,

The Matsubara component of 2.10 is related to the conventional Matsubara Green function of the initial equilibrium state, because the Hamiltonian is constant on the imaginary time branch. It is thus translationally invariant in imaginary time and can be 


\begin{tabular}{|l|l|}
\hline Matsubara Green function & $G^{\mathrm{M}}\left(\tau, \tau^{\prime}\right)=G^{\mathrm{Il}}\left(t_{\min }-i \tau, t_{\min }-i \tau^{\prime}\right)$ \\
\hline \hline Mixed Green function & $G^{\ulcorner}(\tau, t)=G^{\mid+}\left(t_{\min }-i \tau, t\right)=G^{\mid-}\left(t_{\min }-i \tau, t\right)$ \\
\hline & $G^{\urcorner}(t, \tau)=G^{+\mid}\left(t, t_{\min }-i \tau\right)=G^{-\mid}\left(t, t_{\min }-i \tau\right)$ \\
\hline \hline Retarded Green function & $G^{\mathrm{r}}\left(t, t^{\prime}\right)=\Theta\left(t-t^{\prime}\right)\left[G^{-+}\left(t, t^{\prime}\right)-G^{+-}\left(t, t^{\prime}\right)\right]$ \\
\hline Advanced Green function & $G^{\mathrm{a}}\left(t, t^{\prime}\right)=\Theta\left(t^{\prime}-t\right)\left[G^{+-}\left(t, t^{\prime}\right)-G^{-+}\left(t, t^{\prime}\right)\right]$ \\
\hline \hline Lesser Green function & $G^{<}\left(t, t^{\prime}\right)=G^{+-}\left(t, t^{\prime}\right)$ \\
\hline Greater Green function & $G^{>}\left(t, t^{\prime}\right)=G^{-+}\left(t, t^{\prime}\right)=G^{\mathrm{r}}-G^{\mathrm{a}}+G^{<}$ \\
\hline Keldysh Green function & $G^{K}\left(t, t^{\prime}\right)=G^{<}\left(t, t^{\prime}\right)+G^{>}\left(t, t^{\prime}\right)$ \\
\hline \hline Time-ordered Green function & $G^{t}\left(t, t^{\prime}\right)=G^{++}\left(t, t^{\prime}\right)=G^{\mathrm{r}}\left(t, t^{\prime}\right)+G^{<}\left(t, t^{\prime}\right)$ \\
\hline Anti time-ordered Green function & $G^{t}\left(t, t^{\prime}\right)=G^{--}\left(t, t^{\prime}\right)=G^{<}\left(t, t^{\prime}\right)-G^{\mathrm{a}}\left(t, t^{\prime}\right)$ \\
\hline
\end{tabular}

Table 2.2: Parameterization of Contour Green functions. On the right hand side of the relations, the superscript $\alpha$ for each time argument indicates whether the argument is on the upper $(\alpha=+)$, lower $(\alpha=-)$, or imaginary branch of the contour $(\alpha=\mid)$ (cf. Table 2.1). The second equalities follow either directly from the definition (greater component) or from (2.12) [mixed and (anti)time-ordered components].

represented by the Fourier series [37]

$$
\begin{aligned}
& G_{\alpha \alpha^{\prime}}^{\mathrm{M}}\left(\tau, \tau^{\prime}\right)=\frac{i}{\beta} \sum_{n} e^{i \omega_{n}\left(\tau-\tau^{\prime}\right)} g_{\alpha \alpha^{\prime}}^{\mathrm{M}}\left(i \omega_{n}\right) \\
& g_{\alpha \alpha^{\prime}}^{\mathrm{M}}\left(i \omega_{n}\right)=-i \int_{0}^{\beta} d \tau e^{i \omega_{n} \tau} G_{\alpha \alpha^{\prime}}^{\mathrm{M}}(\tau, 0),
\end{aligned}
$$

where $\omega_{n}=(2 n+1) \pi / \beta$ are fermionic Matsubara frequencies. The factor $i$ is inserted to match the conventional definition of equilibrium Matsubara Green functions. In equilibrium, i.e, when the Hamiltonian $H(t) \equiv H\left(t_{\min }\right)$ is time-independent, all two-time functions depend on time difference only and can be represented by their respective Fourier transforms. These Fourier transforms are all related to the Matsubara Green function by analytical continuation: $g_{\alpha \alpha^{\prime}}^{\mathrm{M}}\left(i \omega_{n}\right)$ has a unique analytic continuation $g_{\alpha \alpha^{\prime}}(z)$ to the upper and lower complex half plane with a branch cut

$$
A_{\alpha \alpha^{\prime}}(\omega)=\frac{g_{\alpha \alpha^{\prime}}(\omega-i 0)-g_{\alpha \alpha^{\prime}}(\omega+i 0)}{2 \pi i}
$$

along the real axis. The Green functions are then all related to the spectral function 2.17) and the Fermi function $f(\omega)=1 /\left(e^{\beta \omega}+1\right)$,

$$
\begin{aligned}
& G_{\alpha \alpha^{\prime}}^{\mathrm{r}}\left(t, t^{\prime}\right) \stackrel{e q .}{=}-i \Theta\left(t-t^{\prime}\right) \int d \omega A_{\alpha \alpha^{\prime}}(\omega) e^{-i \omega\left(t-t^{\prime}\right)}, \\
& G_{\alpha \alpha^{\prime}}^{\ulcorner}\left(\tau, t^{\prime}\right) \stackrel{e q .}{=}-i \int d \omega[1-f(\omega)] A_{\alpha \alpha^{\prime}}(\omega) e^{i \omega t^{\prime}-\omega \tau}, \\
& G_{\alpha \alpha^{\prime}}^{<}\left(t, t^{\prime}\right) \stackrel{e q .}{=}+i \int d \omega f(\omega) A_{\alpha \alpha^{\prime}}(\omega) e^{-i \omega\left(t-t^{\prime}\right)} .
\end{aligned}
$$


The corresponding expressions for the advanced and $\neg$ components are not discussed because the symmetry 2.15) holds. Eqs. 2.18) can be proven from an expression of retarded, lesser, and mixed Green function in terms of the Heisenberg operators (cf. Table 2.2),

$$
\begin{aligned}
& G_{\alpha \alpha^{\prime}}^{\mathrm{r}}\left(t, t^{\prime}\right)=-i \Theta\left(t-t^{\prime}\right)\left\langle\left\{\hat{c}_{\alpha}(t), \hat{c}_{\alpha^{\prime}}^{\dagger}\left(t^{\prime}\right)\right\}\right\rangle_{0} \\
& G_{\alpha \alpha^{\prime}}^{\leftarrow}(\tau, t)=-i\left\langle e^{\tau H\left(t_{\min }\right)} c_{\alpha} e^{-\tau H\left(t_{\min }\right)} \hat{c}_{\alpha^{\prime}}^{\dagger}(t)\right\rangle_{0} \\
& G_{\alpha \alpha^{\prime}}^{<}\left(t, t^{\prime}\right)=i\left\langle\hat{c}_{\alpha^{\prime}}^{\dagger}\left(t^{\prime}\right) \hat{c}_{\alpha}(t)\right\rangle_{0} .
\end{aligned}
$$

and the Lehmann representation, i.e., by inserting a complete eigenbasis of $H\left(t_{\min }\right)$ into Eqs. (2.19).

In passing we note a useful interpretation of the various Green function components which arises from Eqs. 2.18) and (2.19): Often the retarded Green function 2.19a) is viewed as a description of the possible excitations of a system (the spectrum), and the lesser components 2.19c as a distribution, i.e., as information about the state of the system. A strict distinction is however exact only for the noninteracting case $2^{2}$ while for an interacting system the spectrum always depends on the distribution.

In nonequilibrium, Green functions depend on both times separately, and thus there is no unique way to represent them by Fourier transform. However, it turns out to be useful to introduce a spectral representation for the mixed component, which describes the memory of the system on correlations in the initial state: For this purpose we start from the partial Fourier transform

$$
\begin{aligned}
G\left\ulcorner\left(i \omega_{n}, t\right)\right. & =\int_{0}^{\beta} d \tau G\left\ulcorner(\tau, t) e^{i \omega_{n} \tau}\right. \\
G\urcorner\left(t, i \omega_{n}\right) & \left.=\int_{0}^{\beta} d \tau G\right\urcorner(t, \tau) e^{-i \omega_{n} \tau} \\
G\ulcorner(\tau, t) & =\frac{1}{\beta} \sum_{n} G\left\ulcorner\left(i \omega_{n}, t\right) e^{-i \omega_{n} \tau}\right. \\
G\urcorner(t, \tau) & =\frac{1}{\beta} \sum_{n} G \neg\left(t, i \omega_{n}\right) e^{i \omega_{n} \tau}
\end{aligned}
$$

(Without loss of generality, fermionic Matsubara frequencies are used, which corresponds to an anti-periodic continuation of $G\ulcorner(\tau, t)$ and $G\urcorner(t, \tau)$ for $\tau<0$ and $\tau>\beta$.) One can then derive a spectral representation of the mixed components by inserting a complete

\footnotetext{
${ }^{2}$ For a quadratic Hamiltonian, $H(t)=\sum_{\alpha \alpha^{\prime}} h_{\alpha \alpha^{\prime}}(t) c_{\alpha}^{\dagger} c_{\alpha^{\prime}}$ the retarded component 2.19a is completely independent of the state, i.e., $\langle\cdots\rangle_{0}$ can be replaced by the expectation value $\langle\psi|\cdots| \psi\rangle$ with respect to an arbitrary state $|\psi\rangle$. This holds because the operator $\left\{c_{\alpha}(t), c_{\alpha^{\prime}}(t)\right\}$ in $2.19 \mathrm{a}$ is a c-number, which can be concluded from the Heisenberg equation of motion for the operator $c_{\alpha}(t)$ : The latter is solved by $c_{\alpha}(t)=\sum_{\bar{\alpha}} U_{\alpha \bar{\alpha}}(t) c_{\bar{\alpha}}$, where the matrix $U(t)=T_{t} \exp \left[-i \int_{0}^{t} d \bar{t} h(\bar{t})\right]$ is the time evolution operator for the single-particle Schrödinger equation. Plugging this into the anticommutator one obtains the c-number $\left\{c_{\alpha}(t), c_{\alpha^{\prime}}(t)\right\}=\sum_{\bar{\alpha}} U(t)_{\alpha \bar{\alpha}} U_{\bar{\alpha} \alpha^{\prime}}^{*}\left(t^{\prime}\right)$, because the equal-time anticommutators are given by $\left\{c_{\alpha}, c_{\alpha^{\prime}}\right\}=\delta_{\alpha \alpha^{\prime}}$.
} 
eigenbasis $|n\rangle$ of $H\left(t_{\min }\right)$ with energies $E_{n}$ in Eq. (2.19b). This gives

$$
\begin{aligned}
G_{\alpha \alpha^{\prime}}^{\ulcorner}(\tau, t) & =-i \sum_{n m} \frac{e^{-\beta E_{n}}}{Z} e^{\tau\left(E_{n}-E_{m}\right)}\left\langle n\left|c_{\alpha}\right| m\right\rangle\left\langle m\left|\hat{c}_{\alpha^{\prime}}^{\dagger}(t)\right| n\right\rangle \\
G_{\alpha \alpha^{\prime}}^{\ulcorner}\left(i \omega_{n}, t\right) & =i \sum_{n m} \frac{e^{-\beta E_{n}}+e^{-\beta E_{m}}}{Z} \frac{\left\langle n\left|c_{\alpha}\right| m\right\rangle\left\langle m\left|\hat{c}_{\alpha^{\prime}}^{\dagger}(t)\right| n\right\rangle}{i \omega_{n}+E_{n}-E_{m}} .
\end{aligned}
$$

Hence $G_{\alpha \alpha^{\prime}}^{\ulcorner}\left(i \omega_{n}, t\right)$, for fixed $t$, has similar analytic properties as $g^{\mathrm{M}}\left(i \omega_{n}\right)$ : It can be analytically continued to the upper and lower half plane, with a branch cut

$$
\begin{aligned}
A_{\alpha \alpha^{\prime}}^{\ulcorner}(\omega, t) & \equiv \frac{G_{\alpha \alpha^{\prime}}^{\ulcorner}(\omega-i 0, t)-G_{\alpha \alpha^{\prime}}^{\ulcorner}(\omega+i 0, t)}{2 \pi i} \\
& =i \sum_{n m} \frac{e^{-\beta E_{n}}+e^{-\beta E_{m}}}{Z}\left\langle n\left|c_{\alpha}\right| m\right\rangle\left\langle m\left|\hat{c}_{\alpha^{\prime}}^{\dagger}(t)\right| n\right\rangle \delta\left(E_{n}-E_{m}+\omega\right) .
\end{aligned}
$$

Analogous expressions follow for $G\urcorner$. The backtransformation 2.20c can then be formulated in terms of a frequency integral by transforming Matsubara sums into real-frequency integrals in the usual way [37]

$$
\begin{gathered}
G_{\alpha \alpha^{\prime}}^{\ulcorner}(\omega, t)=\int d \omega f(\omega) A_{\alpha \alpha^{\prime}}^{\ulcorner}(\omega, t) e^{-\omega \tau} \\
G_{\alpha \alpha^{\prime}}^{\neg}(\omega, t)=\int d \omega f(\omega) A_{\alpha \alpha^{\prime}}^{\neg}(\omega, t) e^{\omega \tau}
\end{gathered}
$$

Note that there is no general symmetry between $G_{\alpha \alpha^{\prime}}^{\ulcorner}(\omega+i 0, t)$ and $G_{\alpha \alpha^{\prime}}^{\ulcorner}(\omega+i 0, t)$ in nonequilibrium, comaparable to $g^{\mathrm{M}}(z)^{*}=g^{\mathrm{M}}\left(z^{*}\right)$ for a real spectrum in equilibrium, and hence the spectrum (2.23) is not purely real. However, the symmetry 2.15) implies

$$
G\ulcorner(z, t)=G\urcorner\left(z^{*}, t\right)^{*}
$$

\subsection{Equations of motion and the self-energy}

In this thesis, the term equations of motion is used for integro-differential equations satisfied by the contour Green function (2.7). In equilibrium, two-time correlation functions depend only on difference in imaginary or real time and are usually parametrized in terms of their respective Fourier transforms. Equations of motion are then simple algebraic relations between the frequency-dependent quantities. In contrast, nonequilibrium two-time correlation functions depend on both time arguments separately. The corresponding equations of motion on the contour $\mathcal{C}$ and the related Dyson equation are often referred to as the (generalized) KB equations (see previous section). Their explicit solution in real time (or rather in the contour time arguments) is the basic technique for the calculation of transient nonequilibrium states. 
The general equation of motion for the one-particle Green function (2.7) is obtained by differentiation of (2.7) with respect to time,

$$
\begin{aligned}
i \partial_{t_{1}} G\left(1,1^{\prime}\right)-[\Lambda \circ G]\left(1,1^{\prime}\right) & =i\left\langle\left[H\left(t_{1}\right), c(1)\right] c^{\dagger}\left(1^{\prime}\right)\right\rangle_{\mathcal{S}}+\delta\left(1,1^{\prime}\right), \\
-i \partial_{t_{1}^{\prime}} G\left(1,1^{\prime}\right)-[G \circ \Lambda]\left(1,1^{\prime}\right) & =i\left\langle c(1)\left[c^{\dagger}\left(1^{\prime}\right), H\left(t_{1}^{\prime}\right)\right]\right\rangle_{\mathcal{S}}+\delta\left(1,1^{\prime}\right) .
\end{aligned}
$$

where $[\Lambda \circ G]\left(1,1^{\prime}\right)=\int d \overline{1} \Lambda(1, \overline{1}) G\left(\overline{1}, 1^{\prime}\right)$ denotes usual matrix multiplication with respect to "space" indices $\alpha$ and contour convolution $\Lambda * G$ with respect to the time arguments, and $\delta\left(1,1^{\prime}\right)=\delta_{\mathcal{C}}\left(t_{1}, t_{1}^{\prime}\right) \delta_{\alpha_{1}, \alpha_{1}^{\prime}}$. The precise definition of the time derivative $\partial_{t}$, the contour delta function $\delta_{\mathcal{C}}\left(t, t^{\prime}\right)$, and the contour-convolution $*$ are given in Table 2.1. Eqs. 2.28a) and $2.28 \mathrm{~b}$ are conjugate to each other, i.e., both contain the same information, and together with the boundary condition (2.11) either of them uniquely determines (2.7). The derivation of Eq. 2.28) is technical but straightforward. The numerical solution of contour equations such as (2.28) is discussed in Sec. 2.5 .

In general, the equations of motion are not closed because $\left\langle\left[H\left(t_{1}\right), c(1)\right] c^{\dagger}\left(1^{\prime}\right)\right\rangle_{\mathcal{S}}$ generates higher-order Green functions if $H(t)$ is not quadratic. Equations of motion for those higher-order Green functions (2.14) take a similar form as Eq. (2.28), and thus lead to an infinite hierarchy of equations for the Green functions of an interacting system 3 The truncation of this hierarchy in various ways generates different approximations. E.g., when the two-particle Green function is decoupled into single-particle ones, the (timedependent) Hartree-Fock approximation is obtained [25]. More sophisticated schemes, based on equations of motion for irreducible quantities, are discussed in Ref. [38.

An important step to develop suitable approximations is the introduction of the selfenergy $\Sigma$. When the Hamiltonian is split into a quadratic part $H_{0}(t)=\sum_{\alpha \alpha^{\prime}} h_{\alpha \alpha^{\prime}}(t) c_{\alpha}^{\dagger} c_{\alpha^{\prime}}$ and an interaction part $W(t)$, Eq. (2.28a) takes the form

$$
\left[G_{0}^{-1} \circ G\right]\left(1,1^{\prime}\right)=i\left\langle\left[W\left(t_{1}\right), c(1)\right] c^{\dagger}\left(1^{\prime}\right)\right\rangle_{\mathcal{S}}+\delta\left(1,1^{\prime}\right)
$$

where $G_{0}^{-1}$ is a differential operator on the contour,

$$
G_{0}^{-1}\left(1,1^{\prime}\right)=\delta\left(1,1^{\prime}\right) i \partial_{t}-\delta_{\mathcal{C}}\left(t_{1}, t_{1}^{\prime}\right) h\left(t_{1}\right)-\Lambda\left(1,1^{\prime}\right)
$$

The term on the right-hand side of Eq. 2.29 , which generates higher-order Green functions, is then formally decoupled through the self-energy $\Sigma\left(1,1^{\prime}\right)$,

$$
[\Sigma \circ G]\left(1,1^{\prime}\right)=i\left\langle\left[W\left(t_{1}\right), c(1)\right] c^{\dagger}\left(1^{\prime}\right)\right\rangle_{\mathcal{S}}
$$

The equation of motion then takes the form of the Dyson equation

$$
\left[\left(G_{0}^{-1}-\Sigma\right) \circ G\right]\left(1,1^{\prime}\right)=\delta\left(1,1^{\prime}\right)
$$

Approximations for the self-energy can be obtained, e.g., by (self-consistent) perturbation theory (cf. Sec 2.4), or via DMFT (Ch. 3).

\footnotetext{
${ }^{3}$ For the noninteracting case with a quadratic action 2.9 , however, equations of motion are closed and provide the most direct way to determine the corresponding Green function.
} 
An important motivation to formulate approximations for the self-energy (instead of the Green function directly) is that there exists a relatively simple criterion by Kadanoff and Baym when the basic conservation laws of energy, particle number, and momentum are satisfied within some approximation for the self-energy [39, 40]: An approximation is conserving in that sense, when $\Sigma\left(1,1^{\prime}\right)$ can be obtained as functional derivative of a functional $\Phi[G]$ of the full Green function

$$
\Sigma[G](1,2)=\frac{\delta \Phi[G]}{\delta G(2,1)} .
$$

The exact functional $\Phi[G]$, from which the exact self-energy could in principle be obtained, is the Luttinger-Ward functional [41]. While the latter is of course unknown, it can be formally constructed from a path integral formulation [42], or term by term in diagrammatic perturbation theory (cf. Sec. 2.4). $\Phi$-derivable approximations in the above sense were originally discussed for equilibrium [39, 40], but the generalization to contour Green functions is straightforward [24]. (Nonequilibrium) DMFT is such a conserving approximation, while any truncation of the perturbation expansion in terms of the noninteracting Green function is not. Using nonconserving approximations for transient nonequilibrium calculations can, e.g., lead to an explicit time-dependent energy altough the Hamiltonian is constant in time. This situation will be encountered, e.g., when the well-known iterated perturbation theory from equilibrium DMFT is used in nonequilibrium DMFT, to calculate the real-time evolution of a nonequilibrium state (cf. Sec. 3.4.3).

\subsection{Perturbation theory}

Perturbation theory in the interaction term of the Hamiltonian is an important tool to generate approximations for many-body systems. Systematic expansions of nonequilibrium Green functions in the interaction were first derived by Kadanoff and Baym [25] and Keldysh [28], and later generalized to the L-shaped contour $\mathcal{C}$ by Danielewicz [33]. Just like for the equilibrium Green functions [37], diagrammatic rules for the perturbation expansion in nonequilibrium are obtained in three steps: First the action (2.9) is divided into a noninteracting part $\mathcal{S}_{0}$ (which is quadratic in field operators) and into an interaction term. The action is then expanded in the latter. This (Taylor) expansion generates higher-order Green functions of the noninteracting system, which are decomposed into products of the single-particle Green function using Wicks theorem. Finally, the manifold of terms that originate from this decomposion is efficiently interpreted in a diagrammatic language.

The crucial point for this scheme is the validity of Wick's theorem. It implies that $n$-particle Green functions (2.14) with a quadratic action $\mathcal{S}_{0}$ factorize into single-particle Green functions. For fermionic particles it reads [37]

$$
\begin{aligned}
\left\langle c(1) \cdots c(n) c^{\dagger}\left(n^{\prime}\right) \cdots c^{\dagger}\left(1^{\prime}\right)\right\rangle_{\mathcal{S}_{0}} & =\sum_{\pi} \operatorname{sgn}(\pi)\left\langle c(1) c^{\dagger}\left(\pi 1^{\prime}\right)\right\rangle_{\mathcal{S}_{0}} \cdots\left\langle c(n) c^{\dagger}\left(\pi n^{\prime}\right)\right\rangle_{\mathcal{S}_{0}} \\
& =\operatorname{det}\left[\left\langle c(i) c^{\dagger}(j)\right\rangle_{\mathcal{S}_{0}}\right]
\end{aligned}
$$


where $\pi$ denotes a permutation of the indices $1^{\prime} \ldots n^{\prime} 4^{4}$ For bosons, no $\operatorname{sign} \operatorname{sgn}(\pi)$ would appear in the first line. The noninteracting Green functions $G_{0}(1,2)=\left\langle c(1) c^{\dagger}(2)\right\rangle_{\mathcal{S}_{0}}$ may be obtained, e.g. by solving the closed equation of motion $\left[G_{0}^{-1} \circ G_{0}\right]\left(1,1^{\prime}\right)=\delta(1,1)$, where $G_{0}^{-1}$ is given by Eq. 2.30). Eq. 2.34 can be proven for contour Green functions, e.g., by comparing the equations of motion on both sides of Eq. (2.34). However, in general it does not hold for time-ordered Green functions such as $\left\langle\psi_{0}\left|T_{t} c(1) \cdots c(n) \times c^{\dagger}\left(n^{\prime}\right) \cdots c^{\dagger}\left(1^{\prime}\right)\right| \psi_{0}\right\rangle$. This led Keldysh to the introduction of the closed contour that includes both forward and backward time evolution.

The above discussion shows that the diagram rules for nonequilibrium Green functions on the contour $\mathcal{C}$ will be exactly the same as those for Matsubara Green functions [37] after imaginary time arguments are replaced by contour time, and time integrals are replaced by contour integrals. We will thus not repeat the derivation of these rules, but instead state the diagrammatic rules for the Hubbard model $(1.1)$ in real space (Table 2.3) for later reference. Nonequilibrium effects can enter both through a possible time-dependence of the interaction $U$, and through the noninteraction Green functions because the action $\mathcal{S}_{0}$ might already include some time-dependent external fields.

Diagrammatic perturbation theory can also be used for a formal power series expansion of the exact Luttinger Ward functional $\Phi[G]$ or its derivative $\Sigma[G]$ [cf. Eq. (2.33)]: $\Sigma[G]$ is given by all skeleton diagrams of the self-energy, i.e., diagrams without self-energy insertions in the internal lines, in which lines are replaced by full interacting Green functions [41]. In Table 2.3d, e.g., diagram (d4) has the topology of a skeleton diagram but not diagram (d3). This construction holds for equilibrium Green functions as well as for nonequilibrium Green functions. Truncation of this skeleton expansion $\Sigma[G]$ can be used to generate conserving approximations in nonequilibrium many-body theory.

\subsection{Differential and integral equations on the contour}

For nonequilibrium problems, it is often neccesary to solve differential equations on the contour $\mathcal{C}$ in real time, rather than in frequency representation. This problem occurs, e.g, in connection with the determination of noninteracting Green functions from Eq. (2.30), the solution of the Dyson equation (2.32), or the calculation of the self-energy from Eq. 2.31). Apart from some exceptional cases such as the interaction quench in the Falicov-Kimball model (Ch. 5), these equations must be solved numerically. This can be implemented in a straightforward way by a discretization of the contour, such that the convolution $*$ and the derivative $\partial_{t}$ turn into corresponding matrix operations [43, 44]. In this work we follow a different approach, which is used more often in the applications of nonequilibrium Green functions (consider, e.g., Refs. [36] and [45] for some recent work). We first parameterize the Green functions in terms of the real and imaginary time components in Table 2.2, and then derive equations for those components (the generalized $\mathrm{KB}$ equations). As we shall see below, this method has the advantage that (i), it can incorporate the symmetries 2.15) of the Green functions from the beginning, and (ii), it

\footnotetext{
${ }^{4}$ It as assumed that anomalous expectation values $\langle c(i) c(j)\rangle_{\mathcal{S}_{0}}$ vanish. The generalization to superconducting states, with $\langle c(i) c(j)\rangle_{\mathcal{S}_{0}} \neq 0$, is straightforward.
} 


\begin{tabular}{|c|c|}
\hline & in oruer cont \\
\hline & $\begin{array}{l}\text { Draw all topologically distinct connected diagrams containing } n \text { vertices } . . . n \\
\text { lines } \ldots \rightarrow \cdots \text { and }(n+1) \text { lines } \longrightarrow \text { (of which two are then external lines). }\end{array}$ \\
\hline 2) & 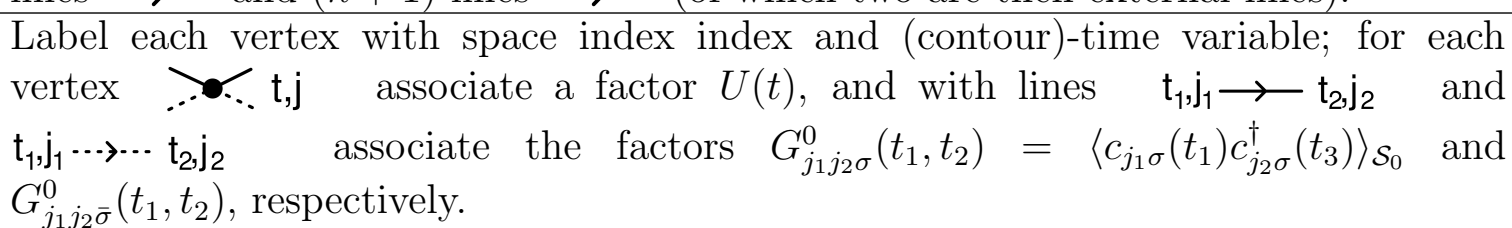 \\
\hline & $\begin{array}{l}\text { Sum over all internal space indices, and integrate internal time variables over the } \\
\text { contour } \mathcal{C} \text {. }\end{array}$ \\
\hline & $\begin{array}{l}\text { Multiply each diagram with an overall factor } i^{n}(-1)^{l} / s \text {, where } l \text { is the number of } \\
\text { closed loops in the diagram, and } s \text { is the symmetry factor, which is given by the } \\
\text { number of ways in which internal vertices can be relabelled without changing the }\end{array}$ \\
\hline
\end{tabular}

b) $n$-th order contribution to the self-energy $\Sigma_{i j \sigma}\left(t, t^{\prime}\right)$

1) Draw all topologically distinct irreducible diagrams containing $n$ vertices ...., $n$ lines $\cdots \rightarrow \cdots$ and $(n-1)$ lines $\longrightarrow$. Irreducible diagrams are those that cannot be separated into two parts by cutting a single line.

2) Label the vertices [the two external vertices are labelled $(i, t)$ and $\left(j, t^{\prime}\right)$ ] and calculate the contribution of each diagram as for the Green function.

c)
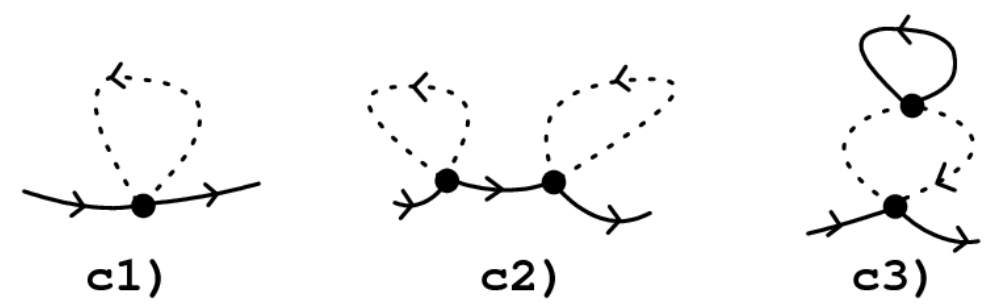

d)
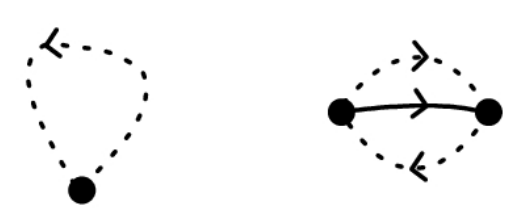

d2)

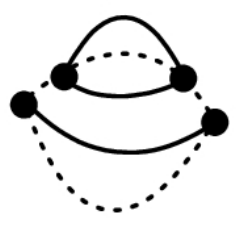

d3)

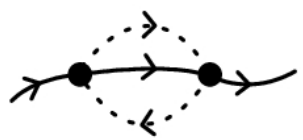

c4)

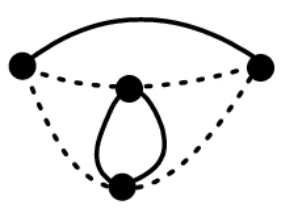

d4)

Table 2.3: Rules for real-space diagrammatic perturbation theory of the Green function (a) and the self-energy (b) of the single-band Hubbard model. (c) shows the first- and secondorder diagrams for the Green function, and (d) shows the first- and second-order diagram for the self-energy [(d1) and (d2), respectively], as well as two fourth-order diagrams. The symmetry factor is 1 for all diagrams apart from (d4), where $s=2$. 
automatically guarantees the causality. The latter is implicit in the contour equations, but rather hidden in (2.28) so that it may easily be violated by approximate methods. Furthermore, we reformulate the KB equations so that we can make direct use of the analytical properties of mixed components introduced in Sec. 2.2.3 this allows for an efficient treatment of initial states at zero temperature.

In the following section two standard problems will be discussed in detail whose solution turns out to be of importance for nonequilibrium DMFT. Both are treated here in scalar notation (i.e., orbital indices are omitted), but their extension to a matrix version is straightforward. To simplify notation, we set $t_{\min }=0$ in the following.

\subsubsection{The inverse of a contour function}

\section{Langreth rules}

We first we discuss the solution of the general equation of motion

$$
\left[i \partial_{t}-h(t)\right] G\left(t, t^{\prime}\right)-[\Lambda * G]\left(t, t^{\prime}\right)=\delta_{\mathcal{C}}\left(t, t^{\prime}\right)
$$

with antiperiodic boundary condition 2.11). Both the equation of motion 2.28a for the noninteracting case, and the scalar version of the Dyson equation $(2.32)$ have this form. To solve Eq. (2.36) we will now derive corresponding differential equations for the Matsubara, retarded, advanced, mixed and lesser components of $G:^{5}$ Translating the derivative term $\partial_{t} G\left(t, t^{\prime}\right)$, the product $h(t) G\left(t, t^{\prime}\right)$, and the delta-function into those components is straightforward from their definition (cf. Table 2.2 and 2.1). To do the same for the convolution $\Lambda * G$, one can use a set of equations which is known as Langreth rules (Table 2.4). (These rules are verified directly by inserting the definition of the various components and of the contour convolution in both sides of the equation.) As a result we get the following equations, which in total are equivalent to Eq. (2.36) and the boundary condition 2.11):

Matsubara component: $(0 \leq \tau \leq \beta)$

$$
\begin{aligned}
& \left(-\partial_{\tau}-h\right) G^{\mathrm{M}}\left(\tau, \tau^{\prime}\right)+i \int_{0}^{\beta} d \bar{\tau} \Lambda^{\mathrm{M}}(\tau, \bar{\tau}) G^{\mathrm{M}}\left(\bar{\tau}, \tau^{\prime}\right)=i \delta\left(\tau-\tau^{\prime}\right) \\
& \text { boundary condition: } G^{\mathrm{M}}\left(0, \tau^{\prime}\right)=-G^{\mathrm{M}}\left(\beta, \tau^{\prime}\right)
\end{aligned}
$$

Retarded/advanced component: $\left(t>t^{\prime}\right)$

$$
\begin{aligned}
& {\left[i \partial_{t}-h(t)\right] G^{\mathrm{r}}\left(t, t^{\prime}\right)-\int_{t^{\prime}}^{t} d \bar{t} \Lambda^{\mathrm{r}}(t, \bar{t}) G^{\mathrm{r}}\left(\bar{t}, t^{\prime}\right)=0,} \\
& \text { initial condition: } \quad G^{\mathrm{r}}(t, t)=-i .
\end{aligned}
$$

\footnotetext{
${ }^{5}$ When the symmetry 2.15 holds, one can restrict oneself to the Matsubara, retarded, $\ulcorner$, and lesser component only.
} 


\begin{tabular}{|c|c|}
\hline a) Convolution $c\left(t, t^{\prime}\right)=[a * b]\left(t, t^{\prime}\right)$ & b) Products $c\left(t, t^{\prime}\right)=a\left(t, t^{\prime}\right) b\left(t^{\prime}, t\right)$ \\
\hline$c^{\mathrm{M}}=a^{\mathrm{M}} \bullet b^{\mathrm{M}}$ & $c^{\mathrm{M}}=a^{\mathrm{M}} b^{\mathrm{M}}$ \\
\hline$=a^{\mathrm{r}} \circ b^{\mathrm{r}}$ & $=a^{\mathrm{r}} b^{<}+a^{<} b^{\mathrm{a}}=a^{\mathrm{r}} b^{>}+a^{>} b^{\mathrm{a}}$ \\
\hline$c^{\mathrm{a}}=a^{\mathrm{a}} \circ b^{\mathrm{a}}$ & $=a^{\mathrm{a}} b^{<}+a^{<} b^{\mathrm{r}}=a^{\mathrm{a}} b^{>}+a^{>} b^{\mathrm{r}}$ \\
\hline$\left.c\urcorner=a\urcorner \circ b^{\mathrm{M}}+a^{\mathrm{r}} \bullet b\right\urcorner$ & $=a\ulcorner b\urcorner$ \\
\hline$c^{\ulcorner}=a^{\ulcorner} \circ b^{\mathrm{a}}+a^{\mathrm{M}} \bullet b^{\ulcorner}$ & $=a\urcorner b\ulcorner$ \\
\hline$=a^{<} \circ b^{\mathrm{a}}+a^{\mathrm{r}} \circ b^{<}+a^{\neg} \bullet b^{\ulcorner}$ & $c^{<}=a^{<} b^{>}$ \\
\hline$c^{>}=a^{>} \circ b^{\mathrm{a}}+a^{\mathrm{r}} \circ b^{>}+a^{\neg} \bullet b^{\ulcorner}$ & $a^{>} b^{<}$ \\
\hline
\end{tabular}

Table 2.4: (a) Langreth rules for the real- and imaginary-time components of the convolution $c$ of two contour functions $a$ and $b$ [24]. In this table, $\circ$ and $\bullet$ denote the convolutions along real and imaginary branches of $\mathcal{C},[a \bullet b]\left(t, t^{\prime}\right)=-i \int_{0}^{\beta} d \bar{\tau} a(t, \bar{\tau}) b\left(\bar{\tau}, t^{\prime}\right)$ and $[a \circ b]\left(t, t^{\prime}\right)$ $=\int_{0}^{t_{\max }} d \bar{t} a(t, \bar{t}) b\left(\bar{t}, t^{\prime}\right)$. (b) Corresponding rules for the product of two contour functions.

$$
\begin{aligned}
& {\left[i \partial_{t^{\prime}}-h\left(t^{\prime}\right)\right] G^{\mathrm{a}}\left(t^{\prime}, t\right)-\int_{t^{\prime}}^{t} d \bar{t} \Lambda^{\mathrm{a}}\left(t^{\prime}, \bar{t}\right) G^{\mathrm{a}}(\bar{t}, t)=0,} \\
& \text { initial condition: } \quad G^{\mathrm{a}}\left(t^{\prime}, t^{\prime}\right)=i
\end{aligned}
$$

Mixed components: $(t>0)$

$$
\left.\left.\left.\left[i \partial_{t}-h(t)\right] G\right\urcorner(t, \tau)-\int_{0}^{t} d \bar{t} \Lambda^{\mathrm{r}}(t, \bar{t}) G\right\urcorner(\bar{t}, \tau)=-i \int_{0}^{\beta} d \bar{\tau} \Lambda\right\urcorner(t, \bar{\tau}) G^{\mathrm{M}}(\bar{\tau}, \tau),
$$

initial condition: $G\urcorner(0, \tau)=G^{\mathrm{M}}(0, \tau)$.

$$
\left(-\partial_{\tau}-h\right) G^{\ulcorner}(\tau, t)-\int_{0}^{t} d \bar{t} \Lambda^{\ulcorner}(\tau, \bar{t}) G^{\mathrm{a}}(\bar{t}, t)=-i \int_{0}^{\beta} d \bar{\tau} \Lambda^{\mathrm{M}}(\tau, \bar{\tau}) G\ulcorner(\bar{\tau}, t),
$$

boundary condition: $\quad G^{\ulcorner}(\beta, t)=-G^{\ulcorner}(0, t)-G^{\mathrm{a}}(0, t)$.

Lesser components: $(t>0)$

$$
\begin{aligned}
& {\left[i \partial_{t}-h(t)\right] G^{<}\left(t, t^{\prime}\right)-\int_{0}^{t} d \bar{t} \Lambda^{\mathrm{r}}(t, \bar{t}) G^{<}\left(\bar{t}, t^{\prime}\right)=} \\
& \left.\quad-i \int_{0}^{\beta} d \bar{\tau} \Lambda\right\urcorner(t, \bar{\tau}) G^{\ulcorner}\left(\bar{\tau}, t^{\prime}\right)+\int_{0}^{t^{\prime}} d \bar{t} \Lambda^{<}(t, \bar{t}) G^{\mathrm{a}}\left(\bar{t}, t^{\prime}\right), \\
& \text { initial condition: } \quad G^{<}\left(0, t^{\prime}\right)=-G^{\ulcorner}\left(\beta, t^{\prime}\right) .
\end{aligned}
$$

Several remarks on these equations and their solution are at order:

(A) Boundary and initial conditions. - In principle, an inhomogeneity $\delta\left(t-t^{\prime}\right)$ and $\delta\left(t^{\prime}-t\right)$ occurs on the right hand side of 2.38a and 2.39a), respectively. When we 
then integrate the differential equation over $t=t^{\prime}$ and use the fact that $G^{\mathrm{r}}\left(t, t^{\prime}\right)$ and $G^{\mathrm{a}}\left(t^{\prime}, t\right)$ vanish for $t>t^{\prime}$, we get the initial conditions (2.38b) and 2.39b), respectively. The boundary and initial conditions of the other components are derived in the following way: 2.37b follows from 2.11) and 2.12); 2.40b and 2.42b) follow because $G$ must be continuous on $\mathcal{C}$. Eq. (2.41b) follows from (2.11), continuity of the contour Green function, and the definition of $G^{\mathrm{a}}$ in Table 2.2 .

(B) Decoupling of initial state: - Eq. 2.37) is independent of all other components. In fact, this must be true due to causality: the Matsubara component is nothing but the usual Matsubara Green function of the initial equilibrium state, which cannot depend on anything that happens at time $t>0$. Furthermore, Eqs. 2.37$)$ to $(2.42)$ can be solved successively: (2.37), (2.38), and (2.39) are each independent and are solved first. Their solution enters Eq. (2.41) and 2.40) for the mixed components both in the initial condition and in the source term. The mixed components finally enter Eq. (2.42), which is solved last.

(C) Causality: - The limits of the various real-time integrals take into account that retarded (advanced) functions vanish when their first time argument is earlier (later) than the second one. One can thus see that Eqs. 22.38), (2.39), (2.40), and (2.42) are initial value problems in which the time derivatives $\partial_{t} G^{x}\left(t, t^{\prime}\right)$ are determined by quantities that depend either on imaginary time (corresponding to the memory of the initial state), or on real time $\bar{t}$ which is earlier than either $t$ or $t^{\prime}$. Eq. (2.41) is a boundary-value problem, where the source term depends only on times $\bar{t} \leq t$. Hence Eqs. (2.37) through (2.42) lead to causal behavior. In contrast, when Eq. (2.36) is solved directly by discretizing the whole contour [44, its causal structure remains hidden and may be violated by numerical errors. It is particularly important to preserve causality in a numerical solution when the input $\Lambda\left(t, t^{\prime}\right)$ itself is less accurate at larger times. This is often the case when $\Lambda\left(t, t^{\prime}\right)$ is obtained numerically, e.g., by means of quantum Monte Carlo methods (Sec. 3.4.2).

(D) Conjugate equation: - Eq. (2.36) is equivalent to the conjugate equation

$$
\left[-i \partial_{t^{\prime}}-h\left(t^{\prime}\right)\right] G\left(t, t^{\prime}\right)-[G * \Lambda]\left(t, t^{\prime}\right)=\delta_{\mathcal{C}}\left(t, t^{\prime}\right)
$$

which is just the fact that two matrices commute when they are inverses of each other. The conjugate equation can be written for the components in the same way as Eq. (2.36). For the retarded, advanced, and lesser component the corresponding equations follow from (2.38), 2.39), and 2.42 by straightforward replacements. The corresponding equations for the mixed components $\neg$ and $\ulcorner$ are thereby essentially exchanged:

Mixed components - conjugate equation: $(t>0)$

$$
\begin{aligned}
& {\left[-i \partial_{t}-h(t)\right] G\left\ulcorner(\tau, t)-\int_{0}^{t} d \bar{t} G\left\ulcorner(\tau, \bar{t}) \Lambda^{\mathrm{a}}(\bar{t}, t)=-i \int_{0}^{\beta} d \bar{\tau} G^{\mathrm{M}}(\tau, \bar{\tau}) \Lambda\ulcorner(\bar{\tau}, t)\right.\right.} \\
& \text { initial condition: } \quad G\left\ulcorner(\tau, 0)=G^{\mathrm{M}}(0, \tau) .\right.
\end{aligned}
$$

$$
\left.\left.\left.\left(\partial_{\tau}-h\right) G\right\urcorner(t, \tau)-\int_{0}^{t} d \bar{t} G^{\mathrm{r}}(t, \bar{t}) \Lambda\right\urcorner(\bar{t}, \tau)=-i \int_{0}^{\beta} d \bar{\tau} G\right\urcorner(t, \bar{\tau}) \Lambda^{\mathrm{M}}(\bar{\tau}, \tau)
$$

boundary condition: $G\urcorner(t, \beta)=-G\urcorner(t, 0)-G^{\mathrm{r}}(t, 0)$. 
(E) Symmetries: - When the symmetry 2.15 is assumed for $\Lambda\left(t, t^{\prime}\right)$, it also holds for the solution $G\left(t, t^{\prime}\right)$. This follows by taking the complex conjugate of Eqs. (2.38), (2.40), and (2.42) and inserting (2.15), after which the equations reduce to the conjugate equations of (2.39), 2.41), and (2.42), respectively.

\section{Transformation from imaginary time to real frequencies}

In the following we assume that $\Lambda^{\mathrm{M}}\left(\tau, \tau^{\prime}\right)$ can be represented in the form 2.16a), and the partial Fourier transforms (2.20a) and 2.20b) of the mixed components have the analytical properties mentioned above Eq. (2.23). As discussed in Sec. 2.2.3, these properties follow from the Lehmann representation for any physical $n$-particle Green functions such as 2.10). We will now see that they are preserved by Eqs. 2.28 and 2.31.

When these properties are assumed, it can be of advantage to perform the partial Fourier transform with respect to imaginary time, in particular for zero initial temperature, $\beta \rightarrow \infty$. When we use the Matsubara frequency representation (2.16a) for $\Lambda^{\mathrm{M}}$ in (2.37), we immediately see that $G$ is of the same form, with

$$
g^{\mathrm{M}}\left(i \omega_{n}\right)=\frac{1}{i \omega_{n}+h-\lambda^{\mathrm{M}}(i \omega)} .
$$

This just confirms that $G^{\mathrm{M}}$ is proportional to the conventional Matsubara Green function of the initial equilibrium state. The partial Fourier transform (2.20b) for Eq. 2.40) and its conjugate 2.45 then give

$$
\begin{aligned}
& \left.\left.\left.\left[i \omega_{n}-h(t)\right] G\right\urcorner\left(t, i \omega_{n}\right)+\int_{0}^{t} d \bar{t} \Lambda^{\mathrm{r}}(t, \bar{t}) G\right\urcorner\left(\bar{t}, i \omega_{n}\right)=\Lambda\right\urcorner\left(t, i \omega_{n}\right) g^{\mathrm{M}}\left(i \omega_{n}\right), \\
& \text { initial condition: } G\urcorner\left(0, i \omega_{n}\right)=-g^{\mathrm{M}}\left(i \omega_{n}\right) . \\
& \left.\left.\qquad\left[i \omega_{n}-h-\lambda^{\mathrm{M}}\left(i \omega_{n}\right)\right] G\right\urcorner\left(t, i \omega_{n}\right)=\int_{0}^{t} d \bar{t} G^{\mathrm{r}}(t, \bar{t}) \Lambda\right\urcorner\left(\bar{t}, i \omega_{n}\right)+G^{\mathrm{r}}(t, 0),
\end{aligned}
$$

Eqs. 2.40) and (2.45) transform in an analogous way and are not given here. The Fourier representation must be taken into account as the mixed components are inserted into Eq. 2.42 for $G^{<}$. The source on the right hand side of Eq. 2.42a reads

$$
\left.\int_{0}^{\beta} d \bar{\tau} \Lambda\right\urcorner(t, \bar{\tau}) G\left\ulcorner(\bar{\tau}, t)=\frac{1}{\beta} \sum_{n} \Lambda\right\urcorner\left(t, i \omega_{n}\right) G\left\ulcorner\left(i \omega_{n}, t\right),\right.
$$

and the initial condition

$$
G^{<}\left(0, t^{\prime}\right)=\frac{1}{\beta} \sum_{n} G^{\ulcorner}\left(i \omega_{n}, t^{\prime}\right) e^{i \omega_{n} 0^{+}} .
$$

On the level of the Eqs. 2.47) and 2.48 the analytical continuation of $G\urcorner\left(t, i \omega_{n}\right)$ to the upper and lower complex half plane is simply achieved by replacing $i \omega_{n} \rightarrow z$. In 
practice, when spectra of the initial state are known for real frequencies, we solve (2.47) or 2.48) and their conjugates for frequencies $z=\omega \pm i 0$. In general $G\urcorner(t, \omega \pm i 0)$ are not related by any symmetry. The source term $(2.49)$ and the initial condition $(2.50)$ are then obtained by replacing Matsubara sums by real-frequency integrals in the usual manner [37,

$$
\begin{aligned}
&\left.\frac{1}{\beta} \sum_{n} \Lambda\right\urcorner\left(t, i \omega_{n}\right) G\left\ulcorner\left(i \omega_{n}, t^{\prime}\right)\right.= \\
&=\int \frac{d \omega}{2 \pi i} f(\omega)[\Lambda\urcorner(t, \omega+i 0) G\left\ulcorner\left(\omega+i 0, t^{\prime}\right)-\Lambda\right\urcorner(t, \omega-i 0) G\left\ulcorner\left(\omega-i 0, t^{\prime}\right)\right], \\
& G^{<}\left(0, t^{\prime}\right)=\int \frac{d \omega}{2 \pi i} f(\omega)[G\ulcorner(\omega+i 0, t)-G\ulcorner(\omega-i 0, t)] .
\end{aligned}
$$

\section{Numerical solution}

In all applications presented later in this thesis, Eq. (2.36) is solved in the real-frequency representation for the mixed components. Frequency integrals $(2.51)$ and $(2.52)$ involve only bounded functions on compact intervals (when the bandwidth of the initial state spectrum is finite) and hence are straightforward to perform numerically. Eqs. (2.38), (2.39), 2.47), and 2.42 take the form of an integro-differential equation of Volterra type 46

$$
\frac{d}{d t} y(t)+p(t) y(t)=\int_{0}^{t} d \bar{t} k(t, \bar{t}) y(\bar{t})+q(t), \quad y(0)=y_{0}
$$

when they are written for fixed second-time argument of the unknown functions. The kernel $k\left(x, x^{\prime}\right)$ is related to $\Lambda^{\mathrm{r}}$ or $\Lambda^{\mathrm{a}}$, and the source term $q(t)$ is determined by the right hand side of the equations. Only Green functions enter $q(t)$ that have already been determined. The Volterra-type structure (2.53) is a particularly clear manifestation of the causality inherent in the original Eq. 2.36). Mathematically, Volterra equations are closely related to ordinary differential equations, and there exist several efficient ways for their numerical solution [46]. For the results of this thesis we have used an equidistant time-grid $t_{n}=n \Delta t, n \leq N_{\max }=t_{\max } / \Delta t$, and approximated the differential and the integral such that the overall discretization error of the solution scales as $\left.\mathcal{O}\left(\Delta t^{p}\right)\right]^{6}$ The algorithm is very stable. Its main limitation is that $\Lambda^{x}\left(t_{i}, t_{j}\right)$ and $G^{x}\left(t_{i}, t_{j}\right)$ must be kept in memory at all previous times $(i, j \leq n)$ in order to proceed from the solution at $t=t_{n}$ to $t=t_{n+1}$. (A maximum of 12228 time steps was used for the results in this thesis.) To reach large times and high accuracy at the same time, higher-order approximations (up to $p=5$ ) turned out to be crucial (Ch. 7).

\footnotetext{
${ }^{6}$ To solve Eq. (2.53) we use the $p$-th order Gregory integration rules for the integral and and the backward differentiation formulae involving $p+1$ points, as explained, Ch. 3 in Ref. [46].
} 


\subsubsection{Volterra equations of the first and second kind}

A second type of contour equations that must be solved is the determination of $G$ from

$$
m G\left(t, t^{\prime}\right)+[G * \Lambda]\left(t, t^{\prime}\right)=Q\left(t, t^{\prime}\right)
$$

where $m=0$ or 1 . For $m=0$, e.g., this problem occurs when the self-energy is determined from Eq. 2.31). The solution is very similar to (2.36) and we will only give the final form of the equations, using the real-frequency representation for mixed and Matsubara components.

Matsubara component: $z=\omega \pm i 0$

$$
g^{\mathrm{M}}(z)=\frac{q^{\mathrm{M}}(z)}{m+\lambda^{\mathrm{M}}(z)}
$$

Retarded/advanced component: $\left(t>t^{\prime}\right)$

$$
\begin{aligned}
& m G^{\mathrm{r}}\left(t, t^{\prime}\right)-\int_{t^{\prime}}^{t} d \bar{t} \Lambda^{\mathrm{r}}(t, \bar{t}) G^{\mathrm{r}}\left(\bar{t}, t^{\prime}\right)=Q^{\mathrm{r}}\left(t, t^{\prime}\right) \\
& m G^{\mathrm{a}}\left(t^{\prime}, t\right)-\int_{t^{\prime}}^{t} d \bar{t} \Lambda^{\mathrm{a}}\left(t^{\prime}, \bar{t}\right) G^{\mathrm{a}}(\bar{t}, t)=Q^{\mathrm{a}}\left(t^{\prime}, t\right)
\end{aligned}
$$

Mixed components: $(t>0)$

$$
\begin{aligned}
& \left.\left.G\urcorner(t, z)=\frac{1}{m+\lambda^{\mathrm{M}}(z)}\left[\int_{0}^{t} d \bar{t} G^{\mathrm{r}}(t, \bar{t}) \Lambda\right\urcorner(\bar{t}, z)+Q\right\urcorner(t, z)\right] \\
& m G\left\ulcorner(z, t)+\int_{0}^{t} d \bar{t} G\left\ulcorner(z, \bar{t}) \Lambda^{\mathrm{a}}(\bar{t}, t)=Q\ulcorner(z, t)\right.\right.
\end{aligned}
$$

Lesser components: $(t>0)$

$$
\begin{aligned}
& m G^{<}\left(t, t^{\prime}\right)+\int_{0}^{t^{\prime}} d \bar{t} G^{<}(t, \bar{t}) G^{\mathrm{a}}\left(\bar{t}, t^{\prime}\right)=Q^{<}\left(t, t^{\prime}\right)-\int_{0}^{t} d \bar{t} G^{\mathrm{r}}(t, \bar{t}) \Lambda^{<}\left(\bar{t}, t^{\prime}\right)\left(t, t^{\prime}\right)+ \\
& \left.\quad+\int \frac{d \omega}{2 \pi i} f(\omega)[G\urcorner(t, \omega+i 0) \Lambda^{\ulcorner}\left(\omega+i 0, t^{\prime}\right)-G^{\urcorner}(t, \omega-i 0) \Lambda^{\ulcorner}\left(\omega-i 0, t^{\prime}\right)\right] .
\end{aligned}
$$

Just as Eq. (2.36) these equations can be solve successively. When one time argument is fixed, Eqs. 2.56), (2.57), 2.59), and (2.60) take the form of Volterra integral equations of the first $(m=0)$ or second $(m=1)$ kind [46],

$$
m y(t)+\int_{0}^{t} d \bar{t} k(t, \bar{t}) y(\bar{t})=q(t) .
$$

At this point the seemingly innocent factor $m$ makes an important difference. While algorithms for the solution of Volterra equations of the second kind are very similar to those of Volterra integro-differential equations, those for the first kind tend to become unstable, in particular those which are in principle correct up to higher-order in the time discretization [46]. For the determination of the self-energy, it is thus not optimal to use Eq. (2.31) directly, but it is better to derive a form that corresponds to Eq. (2.54) with $m=1$ (cf. Ch. 3). 


\section{Chapter 3}

\section{Nonequilibrium dynamical mean-field theory}

\subsection{Dynamical mean-field theory}

\subsubsection{Introductory remarks}

Dynamical mean-field theory (DMFT) [11] provides a solution for a class of interacting lattice models, for which the Hubbard model is certainly the most prominent example. Within DMFT, the lattice model is mapped onto a single site which is coupled to an uncorrelated environment. This environment and its hybridization with the site must be determined self-consistently. A similar mapping of the lattice to a single site is known from the Weiss mean-field theory for spin systems. However, in contrast to the static mean-field theories, the single site in DMFT can dynamically exchange particles with the environment. Those local quantum fluctuations have a profound influence on the physics of the Hubbard model. Without them, e.g., the potential energy $U\left\langle n_{i \uparrow} n_{i \downarrow}\right\rangle$ can only be changed from its uncorrelated value $U\left\langle n_{i \uparrow}\right\rangle\left\langle n_{j \downarrow}\right\rangle$ due to symmetry breaking, and quantum fluctuations are thus crucial to understand the stability and the physical properties of the paramagnetic phase in particular. For strong interactions electrons can become localized and undergo a Mott transition to a paramagnetic insulator, which is not accessible in static mean-field theory, but has been studied extensively within DMFT [11].

DMFT was derived as an exact solution of the Falicov-Kimball model [47] and later the Hubbard model [48] in the limit of infinite dimensions, shortly after this limit had been introduced to correlated lattice fermions by Metzner and Vollhardt [49]. However, the real success of DMFT is based on its application to three-dimensional lattices. 11 It is then only approximate, but from its exact limit it inherits a number of very important properties that any useful nonperturbative approximation should satisfy: (i) DMFT becomes exact both in the noninteracting limit of zero interaction and in the atomic limit of zero hopping amplitudes. This is closely related to the fact that the limit of infinite dimensions preserves the competition between kinetic energy which governs the physics of the Hubbard model.

\footnotetext{
${ }^{1}$ DMFT fails for low-dimensional systems, where spatial fluctuations are important. Various extensions of DMFT have been developed that replace the single-site effective model by a finite cluster [50, 51].
} 
(ii) DMFT is formulated directly in the thermodynamic limit and is therefore free from finite-lattice effects. (iii) The theory is conserving in the sense of Kadanoff and Baym (Sec. 2.28), and thermodynamically consistent. (iv) DMFT is very flexible and thus can be applied to realistic models for solids, such as multi-band Hubbard models with more complicated (local) interaction terms than the Hubbard interaction. Already a brief overview of the application of DMFT in this field would go beyond the scope of this work (for an introduction, consider Refs. [52, 53]). Further generalizations of DMFT include its application to spatially inhomogeneous systems [54], bosonic systems [55], and to nonequilibrium situations which is the main topic of this thesis.

The properties (i)-(iii) mentioned in the previous paragraph, and the mathematical formulation of DMFT can be understood from the limit of infinite dimensions in which DMFT becomes exact. This limit is defined in such a way that the lattice coordination number $Z$ becomes infinite, while the hopping matrix elements have to be scaled such that the kinetic energy stays finite [49]. For example, for the case of nearest-neighbor hopping, hopping matrix elements are given by

$$
t_{i j}=\frac{t_{i j}^{*}}{\sqrt{Z}}
$$

where $t_{i j}^{*}$ is constant as $Z \rightarrow \infty$. For another scaling, either the kinetic or the potential energy would dominate in the limit $Z \rightarrow \infty$, and the limit of infinite dimensions would only lead to trivial solutions of the Hubbard model. ${ }^{2}$ Müller-Hartmann [56, 57] then showed that the scaling (3.1) leads to simplifications for the diagrammatic perturbation theory for the self-energy; this quantity becomes $\boldsymbol{k}$-independent or, more generally, diagonal in real-space,

$$
\Sigma_{i j \sigma}(\omega)=\delta_{i j} \Sigma_{i \sigma}(\omega)
$$

(In symmetry-broken phases or inhomogeneous situations, $\Sigma_{i \sigma}$ depends on the lattice site $i$.) These simplifications of the perturbation expansion have been applied to a direct weak-coupling treatment of the Hubbard model [58, 59]. Further use of the limit of infinite dimensions can be made, e.g., in the evaluation of Gutzwiller expectation values [49] and for disordered systems, where the coherent potential approximation becomes exact [60]. A comprehensive review has been given by Vollhardt in Ref. [61.

The simplifications that arise in the diagrammatic expansion of the self-energy for the limit $Z \rightarrow \infty$ can be taken as a starting point for DMFT [47] (cf. Sec. 3.1.2). Since the diagram rules for perturbation theory are analogous for Keldysh and Matsubara Green functions, Eq. (3.2) holds also for nonequilibrium Keldysh Green functions. It was then first noted by Schmidt and Monien [62] that the self-consistent mapping of lattice models to a single-site problem can also be formulated in terms of Keldysh Green functions. In

\footnotetext{
${ }^{2}$ For bosonic systems it is more difficult to define the limit of infinite dimensions because a third phase (the Bose-Einstein condensate) comes into the play: No scaling of the Bose-Hubbard Hamiltonian can be defined such that the potential energy due to the local repulsion, the kinetic energy of the normal phase and the energy of the condensate remain of the same order for $Z \rightarrow \infty$ [55. For (3.1), e.g., the energy of the condensate diverges, while a scaling with $t \sim 1 / Z$ leads to a vanishing of the normal-phase kinetic energy. This puzzle is resolved in Ref. [55].
} 
particular, nonequilibrium DMFT (as an approximation to finite-dimensional lattices) has the same desirable properties (i)-(iii) that have been discussed for the equilibrium version. Practically, however, the solution of DMFT is completely different for nonequilibrium and equilibrium situations.

The nonequilibrium case was systematically taken up by Freericks and coworkers. They first studied noninteracting electrons in a homogeneous electrical field in the limit of infinite dimensions [63], where Bloch oscillations arise as an AC-response on a DCelectrical field. The same situation was then investigated for the interacting case within the Falicov-Kimball model, using nonequilibrium DMFT [43, 44]. In several other papers a detailed comparison of these results was made to usual Keldysh perturbation theory [64, the relation to the quantum Boltzmann equation was clarified [65], and sum rules for the nonequilibrium retarded self-energy were derived [66]. The nonequilibrium formalism was also used to calculate the equilibrium $f$-electron Green function of the Falicov-Kimball model, which is not easy to obtain otherwise [67]. In the meantime, nonequilibrium DMFT (combined with Floquet theory) was used to study quasi-stationary states that emerge when the system is driven by strong time periodic external fields [68, 69]. In this context, dissipation to a heat bath has been included in the formalism recently [70], which is crucial to define the final steady state without reference to the initial state. While the investigation of steady states continues the original work by Schmidt and Monien, this thesis is concerned with the application of DMFT to the transient dynamics after and during some perturbation, which includes the exact solution of the Falicov-Kimball model after an interaction quench (Ch. 5, Ref. [71]), the description of time-resolved spectroscopy within DMFT (Ch. 8, Refs. [72, 73]), and finally the first DMFT analysis of the transient behavior in the Hubbard model (Ch. 6, Ref. [74]). The remainder of this Chapter is intended to give a detailed description DMFT for the transient-state time evolution.

\subsubsection{DMFT for Keldysh-contour functions}

In the following, DMFT is formulated for the generalized Hubbard model (1.1), which is in thermal equilibrium for times $t<0$. We denote the average over the initial grandcanonical ensemble at temperature $T=1 / \beta$ and chemical potential $\mu$ by $\langle\cdots\rangle_{0}=$ $\operatorname{Tr}\left[e^{-\beta(H(0)-\mu N)} \cdots\right] / Z$, and operators with hat are in Heisenberg picture with respect to the possibly time-dependent Hamiltonian (1.1) [cf. Eq. (2.10]. For example, the contour-ordered real-space lattice Green function is denoted by

$$
G_{i j \sigma}\left(t, t^{\prime}\right)=-i\left\langle\mathrm{~T}_{\mathcal{C}} \hat{c}_{i \sigma}(t) \hat{c}_{j \sigma}^{\dagger}\left(t^{\prime}\right)\right\rangle_{0}
$$

Within the formal language used in this section we do not have to distinguish between the nonequilibrium case, where Green functions have time arguments on the L-shaped Keldysh contour $\mathcal{C}$ (Sec. 2.2), and conventional equilibrium DMFT, for which $\mathcal{C}$ contains only the imaginary-time branch.

Local correlation functions in DMFT at a given site $j$ are obtained from an effective 
single-site problem with action

$$
\begin{aligned}
\mathcal{S}^{j} & =\mathcal{S}_{\mathrm{loc}}^{j}+\mathcal{S}_{\mathrm{hyb}}^{j} \\
\mathcal{S}_{\mathrm{loc}}^{j} & =-i \int_{\mathcal{C}} d t H_{\mathrm{loc}}^{j}(t)-i \mu \sum_{\sigma} \int_{\mathcal{C}} d t c_{\sigma}^{\dagger}(t) c_{\sigma}(t) \\
\mathcal{S}_{\mathrm{hyb}}^{j} & =-i \sum_{\sigma \sigma^{\prime}} \int_{\mathcal{C}} d t \int_{\mathcal{C}} d t^{\prime} c_{\sigma}^{\dagger}(t) \Lambda_{\sigma \sigma^{\prime}}^{j}\left(t, t^{\prime}\right) c_{\sigma^{\prime}}\left(t^{\prime}\right) .
\end{aligned}
$$

In this action, $\mathcal{S}_{\text {loc }}^{j}$ contains the dynamics induced due to the local Hamiltonian

$$
H_{\mathrm{loc}}^{j}(t)=U(t)\left(n_{\uparrow}-\frac{1}{2}\right)\left(n_{\downarrow}-\frac{1}{2}\right)
$$

while $\mathcal{S}_{\text {hyb }}^{j}$ describes the hybridization of the site with an environment that replaces the rest of the lattice. In general, the action can depend on the lattice site $j$, e.g., due to an additional site-dependent on-site potential which is not indicated here. The coupling $\Lambda_{\sigma \sigma^{\prime}}^{j}\left(t, t^{\prime}\right)$ must be determined self-consistently. Note that one can always derive an effective local action for any lattice model by tracing out all other sites. However, this local action then contains $n$-particle interaction terms of arbitrary high order in general. In the limit $Z \rightarrow \infty$ these terms vanish for $n>1$ when the scaling (3.1) is used [11], leading to the DMFT action (3.4).

From the action (3.4) the local Green function is obtained as

$$
G_{\sigma}^{j}\left(t, t^{\prime}\right)=-i\left\langle c_{\sigma}(t) c_{\sigma}^{\dagger}\left(t^{\prime}\right)\right\rangle_{\mathcal{S}^{j}}
$$

where the the notation 2.13 is used. The self-energy $\Sigma_{\sigma}^{j}$ of the single-site problem then follows from the Dyson equation (2.32),

$$
\begin{gathered}
{\left[G_{0}^{-1}\left(t, t^{\prime}\right) * G_{\sigma}^{j}\right]\left(t, t^{\prime}\right)-\left[\Sigma_{\sigma}^{j} * G_{\sigma}^{j}\right]\left(t, t^{\prime}\right)=\delta_{\mathcal{C}}\left(t, t^{\prime}\right),} \\
G_{0}^{-1}\left(t, t^{\prime}\right)=\delta_{\mathcal{C}}\left(t, t^{\prime}\right)\left(i \partial_{t}+\mu\right)-\Lambda_{\sigma}^{j}\left(t, t^{\prime}\right),
\end{gathered}
$$

or, equivalently, Eq. 2.31,

$$
\begin{aligned}
& {\left[\Sigma_{\sigma}^{j} * G_{\sigma}^{j}\right]\left(t, t^{\prime}\right)=U(t) \Gamma_{\sigma}^{j}\left(t, t^{\prime}\right),} \\
& \Gamma_{\sigma}^{j}\left(t, t^{\prime}\right)=-i\left\langle c_{\sigma}(t)\left(n_{\bar{\sigma}}(t)-\frac{1}{2}\right) c_{\sigma}^{\dagger}\left(t^{\prime}\right)\right\rangle_{\mathcal{S}^{j}} .
\end{aligned}
$$

The hybridization $\Lambda$ must be determined self-consistently. For this we equate the selfenergy $\Sigma_{\sigma}^{j}\left(t, t^{\prime}\right)$ and the Green function $G_{\sigma}^{j}\left(t, t^{\prime}\right)$ of the single-site problem with the local self energy $\Sigma_{j j \sigma}\left(t, t^{\prime}\right)$ and the local lattice Green function $G_{j j \sigma}\left(t, t^{\prime}\right)$ of the lattice problem,

$$
G_{j j \sigma}\left(t, t^{\prime}\right)=G_{\sigma}^{j}\left(t, t^{\prime}\right), \quad \Sigma_{i j \sigma}\left(t, t^{\prime}\right)=\delta_{i j} \Sigma_{\sigma}^{j}\left(t, t^{\prime}\right) .
$$

The latter two are related by the lattice Dyson equation,

$$
\begin{gathered}
\sum_{j}\left[\left(G_{\text {latt }, 0}^{-1}\right)_{i j \sigma} * G_{j l \sigma}\right]\left(t, t^{\prime}\right)-\left[\Sigma_{i \sigma} * G_{i l \sigma}\right]\left(t, t^{\prime}\right)=\delta_{i j} \delta_{\mathcal{C}}\left(t, t^{\prime}\right), \\
\left(G_{\text {latt }, 0}^{-1}\right)_{i j \sigma}\left(t, t^{\prime}\right)=\delta_{\mathcal{C}}\left(t, t^{\prime}\right)\left[\delta_{i j}\left(i \partial_{t}+\mu\right)-V_{i j \sigma}(t)\right]
\end{gathered}
$$


which closes the self-consistency.

In practice, Eqs. (3.6), (3.7), (3.10), and (3.11) are solved iteratively: Starting from an initial guess of $\Lambda$, one may calculate the self-energy from (3.7) and (3.6), insert it into the lattice Dyson equation (3.11) to obtain an update of the local Green function, which is in turn inserted in Eq. (3.7) to get an improved guess of the hybridization function $\Lambda$. The whole process is iterated until convergence is reached. The self-consistency and the calculation of the single-site properties from the action (3.4) are discussed in more detail in Secions 3.2 and 3.4, respectively. Both the self-consistency and the single-site problem is rather different for the equilibrium and nonequilibrium case.

To conclude this section, let us briefly repeat one version of the argument that DMFT becomes exact in the limit of infinite dimensions: The mapping onto the single-site problem is equivalent to a replacement of the exact skeleton functional $\hat{\Sigma}_{i j \sigma}[G]$ of the Hubbard model by the corresponding functional $\hat{\Sigma}_{\sigma}^{j}$ of the single-site problem [47, 11],

$$
\hat{\Sigma}_{i j \sigma}^{\mathrm{DMFT}}[G]=\delta_{i j} \hat{\Sigma}^{j}\left[G_{j j \sigma}\right]
$$

at least as far as the computation of single-particle quantities is concerned. In fact, the relation $\Sigma_{\sigma}^{j}=\hat{\Sigma}^{j}\left[G_{\sigma}^{j}\right]$ is ensured by the fact that $G_{\sigma}^{j}$ and $\Sigma_{\sigma}^{j}$ are true solutions of the singlesite problem with action (3.4), although we do not know how to evaluate the skeleton functional at an arbitrary Green function. The quadratic term $3.4 \mathrm{c}$ does not have any influence on the skeleton functional, which depends only on the interaction. Brandt and Mielsch [47] noted for the Falicov-Kimball model that the scaling arguments of Ref. [49] actually imply Eq. (3.12) to arbitrarily high order in perturbation theory: in a skeleton diagram for the self-energy in real space, power counting arguments show that in the limit $Z \rightarrow \infty$ only a single term contributes from the sum over all internal lattice indices, namely the one where all lattice sites are equal. As mentioned above, those arguments hold for Keldysh Green functions as well as for Matsubara Green functions, and hence nonequilibrium DMFT is exact in the limit of infinite dimensions. Another important implication of Eq. (3.12) is that it proves DMFT to be a conserving approximation in sense of Kadanoff and Baym (Sec. 2.3), because the exact functional of the single-site problem is of course $\Phi$-derivable.

\subsection{Nonequilibrium DMFT: the self-consistency condi- tion}

In this section we explain in detail how the Weiss field $\Lambda_{\sigma}^{j}\left(t, t^{\prime}\right)$ for the effective single-site action (3.4) is calculated from the local Green function and self-energy. Only the spatially homogeneous case is considered in the following, where local quantities do not depend on the lattice site $j$ (the superscript $j$ is thus omitted). The generalization of nonequilibrium DMFT to spatially inhomogeneous states is straightforward and indeed very interesting, because many nonequilibrium phenomena involve spatially inhomogeneous states. However, the numerical effort for the solution of the lattice Dyson equation, which is an essential step of the self-consistency cycle, would become quite sizeable: In the absence of 
both translational invariance in space and time, Eq. (3.11) is essentially a matrix problem of dimension (number of time steps) $\times$ (number of inequivalent lattice sites), and already for the homogeneous case (all lattice sites equivalent) thousands of CPU-hours can be spent for the repeated solution of the Dyson equation during the DMFT self-consistency iteration [43, 44].

The self-consistency is solved in two steps. First, the local lattice Green function $G_{j j \sigma}\left(t, t^{\prime}\right)$ is calculated from Eq. (3.11), and second, the new Green function $G_{j j \sigma}\left(t, t^{\prime}\right)$ $=G_{\sigma}\left(t, t^{\prime}\right)$ and the self-energy $\Sigma_{\sigma}\left(t, t^{\prime}\right)$ are inserted into Eq. (3.7) to obtain $\Lambda_{\sigma}\left(t, t^{\prime}\right)$. As in equilibrium DMFT, the spatial part of (3.11) can be decoupled from the timeor frequency part by a Fourier transform to momentum $\boldsymbol{k}$. The case of a homogeneous electrical field, where the hopping matrix elements are decorated with time-dependent Peierls-phase factors (1.2), has been discussed in detail by Freericks at al. [63, 65, 44]. Here we focus on the case of time-independent hopping matrix $V_{i j \sigma}$, for which some important additional simplifications can be derived [71] ? $^{3}$

To diagonalize the spacial part of the Dyson equation we introduce eigenvalues $\epsilon_{\boldsymbol{k} \sigma}$ and eigenvectors $\langle i \mid \boldsymbol{k} \sigma\rangle$ of the hopping matrix, $V_{i j \sigma}$, i.e., $\sum_{j} V_{i j \sigma}\langle j \mid \boldsymbol{k} \sigma\rangle=\epsilon_{\boldsymbol{k} \sigma}\langle i \mid \boldsymbol{k} \sigma\rangle$. For a Bravais lattice, $\boldsymbol{k}$ and $\epsilon_{\boldsymbol{k} \sigma}$ are momenta and band-energies, respectively. We use this terminology throughout the thesis, even though the analysis presented here is valid also when the lattice is not a Bravais (e.g., for the Bethe lattice), in which case case $\boldsymbol{k} \sigma$ is just some labelling of the eigenstates of $V_{i j \sigma}$. The momentum resolved Green function is then given by

$$
G_{\boldsymbol{k} \sigma}\left(t, t^{\prime}\right)=-i\left\langle\mathrm{~T}_{\mathcal{C}} \hat{c}_{\boldsymbol{k} \sigma}(t) \hat{c}_{\boldsymbol{k} \sigma}^{\dagger}\left(t^{\prime}\right)\right\rangle_{0}=\sum_{i j}\langle\boldsymbol{k} \sigma \mid i\rangle G_{i j \sigma}\left(t, t^{\prime}\right)\langle j \mid \boldsymbol{k} \sigma\rangle
$$

The Dyson equation (3.11 then reads

$$
\left(i \partial_{t}+\mu-\epsilon_{\boldsymbol{k} \sigma}\right) G_{\boldsymbol{k} \sigma}\left(t, t^{\prime}\right)-\left[\Sigma_{\sigma} * G_{\boldsymbol{k} \sigma}\right]\left(t, t^{\prime}\right)=\delta_{\mathcal{C}}\left(t, t^{\prime}\right)
$$

and the local Green function is given by

$$
G_{\sigma}\left(t, t^{\prime}\right)=G_{j j \sigma}\left(t, t^{\prime}\right)=\sum_{\boldsymbol{k}}|\langle j \mid \boldsymbol{k} \sigma\rangle|^{2} G_{\boldsymbol{k} \sigma}\left(t, t^{\prime}\right)
$$

In Eq. (3.14), momentum $\boldsymbol{k} \sigma$ enters only via the single-particle energy $\epsilon_{\boldsymbol{k} \sigma}$. Hence the lattice summation in Eq. (3.15) can be reduced an integral over a single energy variable

$$
G_{\sigma}\left(t, t^{\prime}\right)=\int d \epsilon \rho_{\sigma}(\epsilon) G_{\epsilon \sigma}\left(t, t^{\prime}\right)
$$

involving the Green function $G_{\epsilon \sigma}\left(t, t^{\prime}\right)=\left.G_{\boldsymbol{k} \sigma}\left(t, t^{\prime}\right)\right|_{\epsilon=\epsilon_{\boldsymbol{k} \sigma}}$ and the local density of states

$$
\rho_{\sigma}(\epsilon)=\sum_{\boldsymbol{k}}|\langle j \mid \boldsymbol{k} \sigma\rangle|^{2} \delta\left(\epsilon-\epsilon_{\boldsymbol{k} \sigma}\right)
$$

at an arbitrary site $j$.

\footnotetext{
${ }^{3} \mathrm{~A}$ global time-dependent factor of all hopping matrix elements is allowed, because it can be transformed into a time dependence of the interaction (Ch. 7).
} 
The determination of the local Green function is now greatly simplified: Numerically, Eq. (3.14) must be solved (using, e.g., the techniques described in Sec. 2.5) for sufficiently many $\epsilon$ such that the integral (3.16) can be performed with sufficient accuracy. When $\rho(\epsilon)$ is restricted to a finite band, typically few 100 are enough. In contrast, for the case of an electrical field the $\boldsymbol{k}$-sum in 3.15 can be reduced only to a two-dimensional energy integral insted of (3.16), and hence Eq. (3.14) must be solved for $\sim 10000 \boldsymbol{k}$-points [43, 44].

In equilibrium DMFT, the self-consistency can be condensed to a single closed equation for a semielliptic density of states,

$$
\rho(\epsilon)=\frac{\sqrt{4 V^{2}-\epsilon^{2}}}{2 \pi V},
$$

which corresponds to nearest-neighbor hopping on the Bethe lattice [75, 76, 77, 78], or a particular kind of long-range hopping on the hypercubic lattice [79]. In the following we prove that a similar relation holds for the nonequilibrium case: For the density of states (3.18) the self-energy can be eliminated from Eqs. (3.7), (3.14), and (3.16) such that one obtains a closed from expression for the Weiss-field,

$$
\Lambda_{\sigma}\left(t, t^{\prime}\right)=V^{2} G_{\sigma}\left(t, t^{\prime}\right)
$$

This closed form of the self-consistency reduces the DMFT self-consistency cycle to repeating the solution of the single site problem (3.6) and the application of (3.19) until convergence is reached. Eq. (3.19) is used for all DMFT calculations presented in this thesis. A proof follows below.

\section{Closed form self-consistency equations}

We will now give a general recipe to construct a class of functions that imply a selfconsistency equations of closed form when they are used as density of states. The semielliptic density of states $(3.19)$ is the simplest example in this class. For this we will first show the following relation for general square matrices $G$ and $Z$ : Suppose the Hilbert transform

$$
g(z)=\int d \epsilon \frac{\rho(\epsilon)}{z-\epsilon}
$$

of $\rho(\epsilon)$ for the complex frequency $z$ satisfies the implicit equation

$$
z g=1+F(g)
$$

where $F(g)=\sum_{n} f_{n} g^{n}$ is an analytical function with real coefficients $f_{n}$. Then the same relation (3.21) holds for general square matrices $Z$ and $G$ which are defined by

$$
\begin{aligned}
G & =\int d \epsilon \rho(\epsilon) G(\epsilon) \\
G(\epsilon) & =(Z-\epsilon)^{-1} .
\end{aligned}
$$


To see this, multiply the equation $(Z-\epsilon) G(\epsilon)=1$ with $\rho(\epsilon)=-\operatorname{Im}[g(\epsilon+i 0)] / \pi$ and integrate over $\epsilon$. Using $(3.22)$, this yields

$$
Z G=1-\frac{1}{\pi} \int d \epsilon \epsilon \operatorname{Im}[g(\epsilon+i 0)] G(\epsilon)
$$

Due to Eq. (3.21) one can replace $\epsilon \operatorname{Im}[g(\epsilon+i 0)]$ by $\operatorname{Im}[F(g(\epsilon+i 0))]$, leading to

$$
Z G=1-\frac{1}{\pi} \sum_{n} f_{n} \int d \epsilon \operatorname{Im}\left[g(\epsilon+i 0)^{n}\right] G(\epsilon) .
$$

It thus remains to prove

$$
G^{n}=-\frac{1}{\pi} \int d \epsilon \operatorname{Im}\left[g(\epsilon+i 0)^{n}\right] G(\epsilon)
$$

for any integer $n$, which is done by induction: The initial step $(n=1)$ follows from the definition 3.22 . For the induction step, consider

$$
\begin{aligned}
(G)^{n+1} & =(G)^{n} G \\
& \stackrel{(i)}{=} \frac{1}{\pi^{2}} \int d \epsilon d \epsilon^{\prime} \operatorname{Im}\left[g(\epsilon+i 0)^{n}\right] \operatorname{Im}\left[g\left(\epsilon^{\prime}+i 0\right)\right] G(\epsilon) G\left(\epsilon^{\prime}\right) \\
& \stackrel{(i i)}{=} \frac{1}{\pi^{2}} \int d \epsilon d \epsilon^{\prime} \operatorname{Im}\left[g(\epsilon+i 0)^{n}\right] \operatorname{Im}\left[g\left(\epsilon^{\prime}+i 0\right)\right] G(\epsilon)\left(\frac{Z-\epsilon+\epsilon^{\prime}-Z}{\epsilon^{\prime}-\epsilon+i 0}\right) G\left(\epsilon^{\prime}\right) \\
& \stackrel{(i i i)}{=} \frac{1}{\pi^{2}} \int d \epsilon d \epsilon^{\prime} \frac{\operatorname{Im}\left[g(\epsilon+i 0)^{n}\right] \operatorname{Im}\left[g\left(\epsilon^{\prime}+i 0\right)\right]}{\epsilon^{\prime}-\epsilon+i 0}\left(G(\epsilon)-G\left(\epsilon^{\prime}\right)\right) \\
& \stackrel{(i v)}{=}-\frac{1}{\pi} \int d \epsilon G(\epsilon)\left(g(\epsilon+i 0)^{n} \operatorname{Im}[g(\epsilon+i 0)]+\operatorname{Im}\left[g(\epsilon+i 0)^{n}\right] g(\epsilon-i 0)\right) \\
& =-\frac{1}{\pi} \int d \epsilon \operatorname{Im}\left[g(\epsilon+i 0)^{n+1}\right] G(\epsilon)
\end{aligned}
$$

In step (i), proposition (3.26) is used. In (ii), the term in braces is just one, and in (iii) Eq. (3.23) is used. In step (iii) one can tehen perform one of the two energy integrals by making use of the spectral form

$$
g(z)^{n}=-\frac{1}{\pi} \int d \epsilon \frac{\operatorname{Im}\left[g(\epsilon+i 0)^{n}\right]}{z-\epsilon}
$$

which is true because $g(z)^{n}$ is analytic in the upper half plain. Summing up the terms in step (iv) and using $g(\epsilon-i 0)=g(\epsilon+i 0)^{*}$ completes the induction.

The proof for square matrices is easily generalized to contour Green functions, which are matrices in contour-time arguments. Making the replacement $Z \rightarrow \delta_{\mathcal{C}}\left(t, t^{\prime}\right)\left(i \partial_{t}+\mu\right)-$ $\Sigma_{\sigma}\left(t, t^{\prime}\right)$ and $G \rightarrow G_{\sigma}\left(t, t^{\prime}\right)$, Eqs. 3.22) and (3.23) replace 3.16) and (3.14), respectively, and it follows that

$$
Z * G_{\sigma}\left(t, t^{\prime}\right)=\delta_{\mathcal{C}}\left(t, t^{\prime}\right)+F\left(G_{\sigma}\right) \equiv \delta_{\mathcal{C}}(t, t)+\sum_{n} f_{n}\left[G_{\sigma}\right]^{n}\left(t, t^{\prime}\right)
$$


where $G^{n}$ is the $\mathrm{n}$-fold convolution $G * \ldots * G$, and $Z\left(t, t^{\prime}\right)=\delta_{\mathcal{C}}\left(t, t^{\prime}\right)\left(i \partial_{t}+\mu\right)-\Sigma\left(t, t^{\prime}\right)$.

Finally, by comparison with Eq. (3.7), one finds the closed self-consistency equation

$$
\Lambda_{\sigma}\left(t, t^{\prime}\right)=\sum_{n} f_{n+1}\left[G_{\sigma}\right]^{n}\left(t, t^{\prime}\right)
$$

for a density of states whose Hilbert transform satisfies (3.21) with a power series $F(g)$. For the semielliptic DOS, e.g., one has $F(g)=(g V)^{2}$, leading to Eq. (3.19). On the other hand, when (3.21) is not a polynomial of finite degree, one can think of a suitable expansion in terms of orthogonal polynomials, e.g., Chebyshev polynomials [80]. This could lead to a very efficient evaluation of the self-consistency, because orthogonal polynomial of $G_{\sigma}$ (which involve $n$-fold convolutions) can be computed recursively.

\subsection{Calculation of observables}

In this section we derive expressions for a number of observables in the Hubbard model (1.1). The expressions hold for the Falicov-Kimball model (Ch. 5) when the hopping for one spin-species is set to zero. We restrict ourselves to a homogeneous state and field-free case.

The density for spin $\sigma$ is given by the local Green function $G_{j j \sigma}\left(t, t^{\prime}\right)$, which is obtained directly from the single-site problem [Eq. 3.10]. using the definition of the lesser Green function in Table 2.2 we have

$$
n_{\sigma}(t)=\frac{1}{L} \sum_{j}\left\langle\hat{c}_{j \sigma}^{\dagger}(t) \hat{c}_{j \sigma}(t)\right\rangle_{0}=-i G_{\sigma}^{<}(t, t)
$$

provided that the state is homogeneous. Here and in the following, $L$ is the lattice size. The particle number is conserved, such that the condition $G_{\sigma}^{<}(t, t)=$ const. provides a first check for numerical data.

The occupation of the single-particle momentum states with spin $\sigma$

$$
n_{\sigma}\left(\epsilon_{\boldsymbol{k} \sigma}, t\right) \equiv\left\langle\hat{c}_{\boldsymbol{k} \sigma}^{\dagger}(t) \hat{c}_{\boldsymbol{k} \sigma}(t)\right\rangle_{0}=-i G_{\boldsymbol{k} \sigma}^{<}(t, t)
$$

is obtained from the momentum resolved Green function (3.13). In the absence of electromagnetic fields, $G_{\boldsymbol{k} \sigma}\left(t, t^{\prime}\right)$ depends on momentom $\boldsymbol{k}$ only via the band-energy $\epsilon_{\boldsymbol{k}}$ and is determined from the lattice Dyson equation (3.14). The kinetic energy per lattice site

$$
E_{k i n}(t)=\frac{1}{L} \sum_{i j \sigma} V_{i j \sigma}\left\langle\hat{c}_{i \sigma}^{\dagger}(t) \hat{c}_{j \sigma}(t)\right\rangle_{0}=\frac{1}{L} \sum_{\boldsymbol{k} \sigma} \epsilon_{\boldsymbol{k} \sigma}\left\langle\hat{c}_{\boldsymbol{k} \sigma}^{\dagger}(t) \hat{c}_{\boldsymbol{k}}(t)\right\rangle_{0},
$$

is obtained from $n(\epsilon, t)$ by replacing the $\boldsymbol{k}$-sum with an integral over the local density of states [Eq. (3.17)],

$$
E_{k i n}(t)=\sum_{\sigma} \int d \epsilon \rho_{\sigma}(\epsilon) \epsilon n_{\sigma}(\epsilon, t) \equiv \sum_{\sigma} E_{k i n}^{\sigma}
$$


In the noninteracting equilibrium state, we have $n_{\sigma}(\epsilon)=f(\epsilon-\mu)$ and

$$
E_{k i n, 0}^{\sigma}=\int d \epsilon \rho_{\sigma}(\epsilon) \epsilon f(\epsilon-\mu)
$$

Next we are interested in the double occupation per lattice site

$$
d(t)=\frac{1}{L} \sum_{i}\left\langle\hat{n}_{i \uparrow}(t) \hat{n}_{i \downarrow}(t)\right\rangle_{0},
$$

and the interaction energy

$$
\begin{aligned}
E_{p o t} & \equiv U(t) \sum_{i}\left\langle\left(\hat{n}_{i \uparrow}(t)-\frac{1}{2}\right)\left(\hat{n}_{i \downarrow}(t)-\frac{1}{2}\right)\right\rangle_{0} \\
& =U(t)\left[d(t)-\frac{1}{2}\left(n_{\uparrow}(t)+n_{\downarrow}(t)\right)+\frac{1}{4}\right] .
\end{aligned}
$$

To calculate this quantity we consider the equation of motion for the local lattice Green function $G_{j j \sigma}$, which reads

$$
\begin{aligned}
& {\left[\left(G_{\text {latt }, 0}^{-1}\right)_{j l \sigma} * G_{l j \sigma}\right]\left(t, t^{\prime}\right)=\delta_{\mathcal{C}}\left(t, t^{\prime}\right)+U(t) \Gamma_{j \sigma}\left(t, t^{\prime}\right),} \\
& \Gamma_{j \sigma}\left(t, t^{\prime}\right)=-i\left\langle\mathrm{~T}_{\mathcal{C}} \hat{c}_{i \sigma}(t)\left(\hat{n}_{i \bar{\sigma}}(t)-\frac{1}{2}\right) \hat{c}_{i \sigma}^{\dagger}\left(t^{\prime}\right)\right\rangle_{0},
\end{aligned}
$$

where $\left(G_{l a t t, 0}^{-1}\right)_{j l \sigma}$ is given by Eq. $3.11 \mathrm{~b}$. Comparison with the dyson equation 3.11 yields

$$
U(t) \Gamma_{j \sigma}\left(t, t^{\prime}\right)=\left[\Sigma_{\sigma} * G_{j j \sigma}\right]\left(t, t^{\prime}\right),
$$

because the self-energy is local and site-independent. Hence $\Gamma_{\sigma} \equiv \Gamma_{j \sigma}$ can be determined from quantities measured in the single-site problem. In fact, the local lattice correlation function $\Gamma_{j \sigma}\left(t, t^{\prime}\right)$ and thus also the double occupancy is the same as the corresponding correlation function in the single-site problem [cf. Eqs. (3.8) and (3.9)], as expected within DMFT. On the other hand, the definition of $\Gamma_{i \sigma}\left(t, t^{\prime}\right)$ implies that for a homogeneous state

$$
d(t)=-i \Gamma_{i \sigma}^{<}(t, t)+\frac{1}{2} n_{\bar{\sigma}}(t)
$$

Finally we can compute the total energy from Eqs. 3.32 and (3.35),

$$
E_{\text {tot }}(t)=E_{\text {kin }}(t)+E_{\text {pot }}(t)
$$

This quantity must be constant when the Hamiltonian is time-independent, which provides a second test for the accuracy of the numerical solution. From the equation of motion 3.37) one can also directly get the expression

$$
\partial_{t} G_{\sigma}^{<}\left(t, t^{\prime}\right)_{t=t^{\prime}}=E_{k i n}^{\sigma}(t)+U(t) d(t)-n_{\sigma}(t)\left[\frac{1}{2} U(t)+\mu\right] .
$$




\subsection{Nonequilibrium DMFT: Impurity solvers}

\subsubsection{Introduction}

The single-site problem of DMFT still represents an interacting many-body problem. Several numerical and approximate analytical tools have been developed to solve this problem for equilibrium situations. In this section we will focus on the Hubbard model and discuss possible extension of those methods to the nonequilibrium case. As we shall see, each single one of those methods encounters a different problem when extended to nonequilibrium. Until recently, nonequilibrium DMFT was therefore only applied to the Falicov-Kimball model [43, 71, 68], for which the single-site action (3.4) can be reduced to a quadratic one (Ch. 5).

In equilibrium DMFT, the single-site action (3.4) is equivalent to that of a singleimpurity Anderson model (SIAM) [48], which describes an interacting impurity orbital coupled to a bath of noninteracting electrons. Several methods that solve the DMFT action in equilibrium are based on this fact, in particular exact diagonalization (ED) [81, 11], numerical renormalization group (NRG) [82, 83, 84] and density matrix renormalization group (DMRG) [85, 86]. Motivated by quantum dot experiments and experiments with cold atomic gases, NRG [87] and DMRG [88, 89] have been generalized to time-dependent models in the past few years. Both methods are therefore possible candidates for an impurity solver in nonequilibrium DMFT, in particular DMRG which is less restricted with respect to the type of time-dependent terms in the Hamiltonian. However, one must first find a way to actually represent the action (3.4) by some time-dependent impurity Hamiltonian. This problem is partly solved in Sec. 3.4.4. As we will see, the mapping in nonequilibrium is quite different from the standard procedure in equilibrium DMFT.

On the other hand, there are some methods which attempt to solve the single-site problem without any reference to an impurity Hamiltonian, mainly (numerically exact) Quantum Monte Carlo methods (QMC), and the iterated perturbation theory (IPT) [90, 11]. Their extension to the nonequilibrium case is described in Sec. 3.4.2 and 3.4.3. respectively. The continuous-time QMC algorithm which is described in Sec. 3.4.2 is the only of the impurity solvers described in this chapter which has currently been applied to nonequilibrium DMFT for the Hubbard model [74].

Apart from these methods, various other approximate analytical methods have been developed for the equilibrium case [11. To judge the potential application of all those methods as approximate impurity solvers for nonequilibrium DMFT is beyond the scope of this thesis.

\subsubsection{Continuous-time Quantum Monte Carlo}

QMC was among the first methods that were used to solve DMFT equations [91, 92, 93]. Because it is numerically exact on the one hand, and the computational effort scales most favorable for the multiband Hubbard models, it is almost exclusively used when DMFT is applied to real materials. The main drawback of QMC is that Green functions are calculated on the imaginary time axis and thus an analytical continuation is needed 
to obtain spectra of real frequencies. Mathematically, this analytical continuation is an ill-conditioned problem, which is solved using maximum-entropy estimation [94].

Originally, mainly the Hirsch-Fye algorithm [95] was used to solve the SIAM with QMC. It relies on a discretization of the imaginary time axis with a fixed time-grid and Hubbard-Stratonovic decoupling of the interaction term [11]. The Hirsch-Fye algorithm for the SIAM does not suffer from the notorious fermionic sign problem at least for the half-filled sigle-site band Hubbard model. However, the numerical effort scales as $\beta^{3}$ and hence low temperatures are inaccessible. Recently, various continuous-time QMC (CTQMC) algorithms have been developed for impurity models. These methods expand the action (3.4) either in the interaction term [96, 97] or in the hybridization [98, 99], and stochastically sum over all contributions. They are now often used in the context of DMFT [100], because they perform better in a large region of parameter space [101] and can be applied even for more general interaction terms which cannot be treated by the Hirsch-Fye algorithm due to a severe sign problem [102].

Recently, several continuous time algorithms have been generalized to the Keldysh contour to treat nonequilibrium impurity problems [103, 104, 105]. All those real-time QMC algorithms unavoidably encounter a "dynamical sign problem" due to the fact that the action (3.4) and hence all weights in the stochastic sum are complex quantities. Usually, this leads to an exponential increase of the numerical effort with the maximum simulated time $t_{\max }$. For small and intermediate times, however, CTQMC has turned out to be very suitable as an impurity solver for nonequilibrium DMFT (Ch. 6, Ref. [74]). In this application the nonequilibrium version [104] of the weak coupling auxiliary-field QMC algorithm by Gull et al. [97] was used. As we will see below, the fact that it is a weak-coupling expansion makes it very suitable to study noninteracting initial states. On the other hand, the dynamical sign problem increases with interaction $U$, such that the maximum time that can be reached scales as $t_{\max } \sim 1 / U$. A brief discussion of the algorithm is presented in the following subsection.

\section{The weak-coupling auxiliary-field CTQMC algorithm}

In weak-coupling auxiliary field QMC, observables and correlation function are computed by expanding expectation values $\left\langle A_{1}\left(t_{1}\right) A_{2}\left(t_{2}\right) \ldots\right\rangle_{\mathcal{S}}$ in the interaction part of the Hamiltonian in the action (3.4b). For the usual Hubbard interaction (3.5), this is chosen as 104]

$$
H_{\text {int }}(t)=U(t)\left(n_{\uparrow}-\frac{1}{2}\right)\left(n_{\downarrow}-\frac{1}{2}\right)-K / t_{\max },
$$

where the free constant $K$ can be chosen to optimize the algorithm. The interaction term is then decoupled by the introduction of auxiliary Ising fields $s= \pm 1$,

$$
\begin{aligned}
H_{\text {int }}(t)= & \frac{K}{2 t_{\max }} \sum_{s= \pm} \exp \left[s \gamma(t)\left(n_{\uparrow}-n_{\downarrow}\right)\right], \\
& \cosh (\gamma)=1+\frac{U(t) t_{\max }}{2 K} .
\end{aligned}
$$

The resulting $n$-particle Green functions of the quadratic action $\mathcal{S}_{0}=\mathcal{S}_{\text {hyb }}-i \mu \sum_{\sigma}$ $\int_{\mathcal{C}} d t n_{\sigma}(t)$ are then factorized using Wick's theorem 2.34. The final expression for the 
partition function $\operatorname{Tr}[\exp (\mathcal{S})]$ can be written in the form [104]

$$
\operatorname{Tr}[\exp (\mathcal{S})]=\sum_{n=0}^{\infty} \frac{i^{n} K^{n}}{t_{\max }^{n} n !} \int_{\mathcal{C}} d t_{1} \ldots \int_{\mathcal{C}} d t_{n} \sum_{s_{1} \ldots s_{n}} \operatorname{det} N_{\uparrow}\left(s_{i}, t_{i}\right) \operatorname{det} N_{\downarrow}\left(s_{i}, t_{i}\right),
$$

where $\left[N_{\sigma}\right]_{i j}$ are matrices that involve noninteracting Green function $G_{\sigma}^{0}\left(t, t^{\prime}\right)=$ $-i\left\langle c_{\sigma}(t) c_{\sigma}^{\dagger}\left(t^{\prime}\right)\right\rangle_{\mathcal{S}_{0}}$. The latter can be determined from the equation of motion (Sec. 2.3

$$
\left(i \partial_{t}+\mu\right) G_{\sigma}^{0}\left(t, t^{\prime}\right)-\left[\Lambda_{\sigma}\left(t, t^{\prime}\right) * G_{\sigma}^{0}\right]\left(t, t^{\prime}\right)=\delta_{\mathcal{C}}\left(t, t^{\prime}\right)
$$

Similar expressions are obtained for the expectation values of observables or the Green function. They are evaluated by a stochastic sum over a configuration space which consists of an arbitrary number of spins $s_{k}$ at arbitrary positions $t_{k}$ on the contour $\mathcal{C}$, and the weight of each configuration is related to the determinants $N_{\sigma}$. The Monte Carlo steps consist in adding, removing, and shifting the spins on $\mathcal{C}$. Note that the dynamical sign problem is already evident in Eq. (3.45): In each term with at least one real time argument, the two contributions to (3.45) where the spin with the largest time is on the upper and on the lower part exactly cancel, due to 2.12 and the definition of the contour integral. After all terms on the real branch are cancelled, the final result of (3.45) is the partition function of the initial equilibrium state, which depends only on the imaginary-time branch.

Further technical details of the algorithm can be taken from the original Ref. [104]. The implementation of this variant of CTQMC as an impurity solver into the DMFT iteration for the solution of an interaction quench in the Hubbard model (Ch. 6) is described in App. A. The detailed discussion given there will also clarify why the weakcoupling expansion is particularly well suited to treat noninteracting initial states: The Monte Carlo sampling has to be done only on the real-time part of the contour, and imaginary time arguments in the mixed and Matsubara Green function components can thus be replaced by partial Fourier transform. This allows to use the special analytical properties of these Fourier transformed functions (Sec. 2.2.3) and thus treat initial states at zero temperature.

\subsubsection{Perturbative solution of the local problem}

The perturbative calculation for the self-energy can be obtained from the rules presented in (2.4). The rules for the single-site model are the same as for the Hubbard model, when the sum over site indices is omitted. Here we compare two approaches: (i) the so-called iterated perturbation theory (IPT), which is a second-order perturbation expansion of $\Sigma_{\sigma}$ in the noninteracting Green function $G_{\sigma}^{0}$, and (ii), self-consistent perturbation theory (SPT), for which the skeleton expansion of the self-energy is evaluated up to second order. We restrict ourselves to the half-filled case, where the first order diagrams vanish (these are the Hartree contributions which give a shift of the chemical potential with respect to $\mu=U / 2$ ), and only one diagram contributes to $\Sigma$, i.e., diagram (d2) in Table. 2.3. Lines in the diagram represent either the full interacting Green function (SPT), or the 

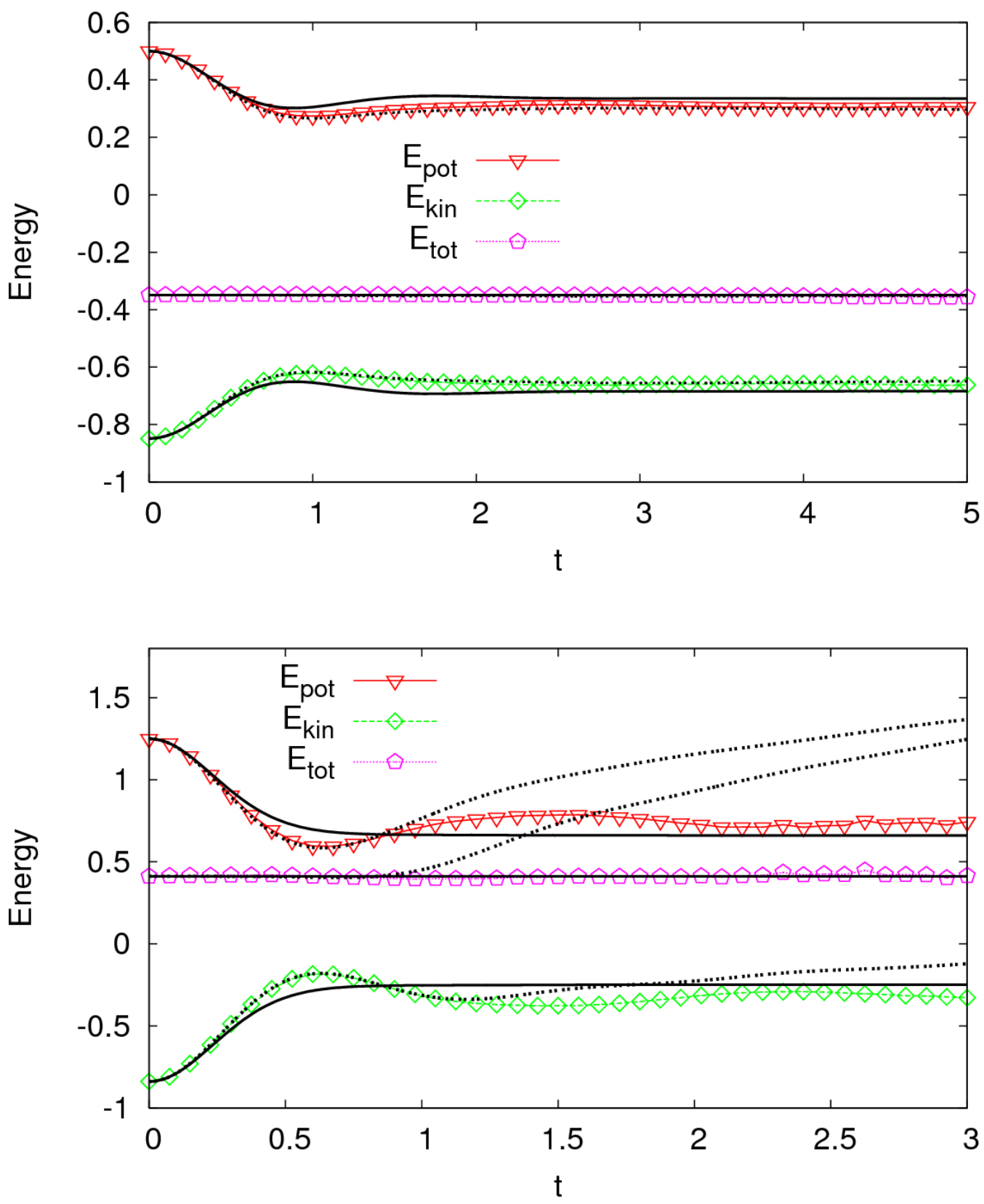

Figure 3.1: Total energy $E_{\text {tot }}=E_{\text {pot }}+E_{\text {kin }}$, potential energy $E_{\text {pot }}=U d(t)$, and kinetic energy $E_{\text {kin }}[\mathrm{Eq} .3 .32 \mathrm{~b}$ in the Hubbard model at $U=2$ (upper panel) and $U=5$ (lower panel). Results are obtained with nonequilibrium DMFT for a semielliptic density of states (3.18), using either IPT (dashed lines), SPT (solid lines) or CTQMC (symbols) as impurity solver. The initial state at $t=0$ is the noninteracting Fermi sea at temperature $T=0$ (upper panel) and $T=0.1$ (lower panel). 
noninteracting Green function $G_{0}$ that is obtained from Eq. (3.46) (IPT),

$$
\begin{aligned}
\Sigma_{\sigma}^{I P T}\left(t, t^{\prime}\right) & =U(t) U\left(t^{\prime}\right) G_{\sigma}^{0}\left(t, t^{\prime}\right) G_{\bar{\sigma}}^{0}\left(t^{\prime}, t\right) G_{\bar{\sigma}}^{0}\left(t, t^{\prime}\right) \\
\Sigma_{\sigma}^{S P T}\left(t, t^{\prime}\right) & =U(t) U\left(t^{\prime}\right) G_{\sigma}\left(t, t^{\prime}\right) G_{\bar{\sigma}}\left(t^{\prime}, t\right) G_{\bar{\sigma}}\left(t, t^{\prime}\right) .
\end{aligned}
$$

Either of these equations can be incorporated easily in the self-consistency scheme.

In equilibrium situations, IPT is frequently used as an approximate impurity solver [90, 11. Although (3.47) is a weak-coupling expansion, it is accidentally correct in the atomic limit for half-filling [11]. In many aspects, IPT thus provides a reasonable extrapolation between the two exact limits $U=0$ and $V=0$. In particular, it qualitatively reproduces the DMFT phase diagram of the Mott transition in the paramagnetic phase, although there are quantitative differences to numerically exact QMC results. Unfortunately, we find that the same is not true for nonequilibrium. It may be that IPT is exact for the trivial case of a quench from the noninteracting case to the atomic limit (this was not checked), but the method fails in the intermediate coupling regime because it is not conserving. This is demonstrated for a specific situation: In Fig. 3.1 we have plotted the internal energy, the potential energy, and the kinetic energy after a sudden switch of the interaction in the Hubbard model from the noninteracting state to $U>0$. Results of IPT and SPT are compared to numerically exact CTQMC results. By construction, both IPT and SPT work well for small $U$ (IPT is actually a bit better), but IPT results clearly violates energy conservation for $U=5$, and leads to completely wrong values of the double occupancy. SPT, on the other hand, is conserving according to the discussion in Sec. 2.4. However, it apparently misses some important oscillations of the time evolution and instead predicts a monotonous decay to a steady state.

\subsubsection{The mapping problem}

Before powerful numerical methods for nonequilibrium, such as time-dependent DMRG, can be applied to the single-site problem of nonequilibrium DMFT, one must find a Hamiltonian which corresponds to the effective action (3.4). Having in mind the equilibrium case [48], an obvious first guess is the SIAM with time-dependent bath parameters,

$$
\begin{aligned}
H_{\mathrm{SIAM}}(t) & =H_{l o c}(t)+H_{b a t h}(t)+H_{h y b}(t), \\
H_{b a t h}(t) & =\sum_{p \sigma} \epsilon_{p \sigma}(t) a_{p \sigma}^{\dagger} a_{p \sigma} \\
H_{h y b}(t) & =\sum_{p \sigma} V_{p \sigma}(t) c_{\sigma}^{\dagger} a_{p \sigma}+\text { h.c. }
\end{aligned}
$$

where $p$ labels the bath degrees of freedom, $a_{p \sigma}\left(a_{p \sigma}^{\dagger}\right)$ are annihilation (creation) operators of fermionic particles in bath states respectively, and $H_{l o c}$ is given by (3.5). In this section we will show that in fact for any given $\Lambda$ (having, of course, the usual properties of the contour function) the bath parameters in $(3.49$ can be chosen in such a way that the effective action for the $c$-electrons equals (3.4). In other words, solving the DMFT singlesite model is equivalent to solving a SIAM with time-dependent bath couplings. However, 
in contrast to the mapping in equilibrium two baths are needed for the nonequilibrium case (or, equivalently, one bath with doubly degenerate energy levels): One describes the fading memory on the initial state, while the other one is building up for $t>0$ and finally describes the steady state of the system at $t=\infty$.

We start by deriving the effective action for the $c$-particles in $(3.49)$, i.e., we determine $\Lambda_{\sigma}\left(t, t^{\prime}\right)$ such that the impurity Green function $G_{\sigma}\left(t, t^{\prime}\right)=-i\left\langle\mathrm{~T}_{\mathcal{C}} \hat{c}_{\sigma}(t) \hat{c}_{\sigma}^{\dagger}\left(t^{\prime}\right)\right\rangle_{0}$ and the impurity self-energy follow from the action (3.4). This could be formulated concisely within a path integral formalism. However, we have not introduced this language and thus prefer to give an equivalent construction using only simple equations of motion. According to Sec. 2.3. the impurity Green function $G_{\sigma}\left(t, t^{\prime}\right)$ satisfies the equation of motion

$$
\left(i \partial_{t}+\mu\right) G_{\sigma}\left(t, t^{\prime}\right)-\sum_{p} V_{p \sigma}(t) F_{p \sigma}\left(t, t^{\prime}\right)=\delta_{\mathcal{C}}\left(t, t^{\prime}\right)+i\left\langle\mathrm{~T}_{\mathcal{C}}\left[c_{\sigma}(t), H_{l o c}(t)\right] \hat{c}_{\sigma}^{\dagger}\left(t^{\prime}\right)\right\rangle,
$$

where $F_{p \sigma}\left(t, t^{\prime}\right)=-i\left\langle\mathrm{~T}_{\mathcal{C}} \hat{a}_{p \sigma}(t) \hat{c}_{\sigma}\left(t^{\prime}\right)^{\dagger}\right\rangle$. Similarly, we obtain an equation of motion for $F_{p \sigma}\left(t, t^{\prime}\right)$,

$$
\left[i \partial_{t}+\mu-\epsilon_{p \sigma}(t)\right] F_{p \sigma}\left(t, t^{\prime}\right)=V_{p \sigma}^{*}(t) G\left(t, t^{\prime}\right) .
$$

With the Green function $g_{p \sigma}\left(t, t^{\prime}\right)$ of a single isolated bath site $p$,

$$
\left[i \partial_{t}+\mu-\epsilon_{p \sigma}(t)\right] g_{p \sigma}\left(t, t^{\prime}\right)=\delta_{\mathcal{C}}\left(t, t^{\prime}\right),
$$

we can then invert Eq. (3.51) and get

$$
F_{p \sigma}\left(t, t^{\prime}\right)=\int_{\mathcal{C}} d \bar{t} g_{p \sigma}(t, \bar{t}) V_{p \sigma}^{*}(\bar{t}) G\left(\bar{t}, t^{\prime}\right)
$$

When this is inserted into Eq. (3.50), one can see by comparison with Eq. 2.28a that $G_{\sigma}\left(t, t^{\prime}\right)$ is also obtained from $(3.4)$, provided that

$$
\Lambda_{\sigma}\left(t, t^{\prime}\right)=\sum_{p} V_{p \sigma}(t) g_{p \sigma}\left(t, t^{\prime}\right) V_{p \sigma}^{*}\left(t^{\prime}\right)
$$

The local Green function of an isolated bath site is easily calculated, either from Eq. (3.52), or directly from $g_{p \sigma}\left(t, t^{\prime}\right)=-i\left\langle\mathrm{~T}_{\mathcal{C}} \hat{a}_{p \sigma}(t) \hat{a}_{p \sigma}^{\dagger}\left(t^{\prime}\right)\right\rangle_{0}$. We get

$$
g_{p \sigma}\left(t, t^{\prime}\right)=i\left[f\left(\epsilon_{p \sigma}-\mu\right)-\Theta_{\mathcal{C}}\left(t, t^{\prime}\right)\right] e^{i \mu\left(t^{\prime}-t\right)} \exp \left(i \int_{0}^{t} d \bar{t} \epsilon_{p \sigma}(\bar{t})-i \int_{0}^{t^{\prime}} d \bar{t} \epsilon_{p \sigma}(\bar{t})\right)
$$

where the integrals are along the contour $\mathcal{C}$. From this expression one can see that the bath energies can always be assumed as time-independent for the mapping to a SIAM: The same hybridization function (3.54) is obtained either for time-dependent energies $\epsilon_{p \sigma}(t)$, or from a different SIAM with constant energies $\tilde{\epsilon}_{p \sigma}=\epsilon_{p \sigma}(0)$ and hybridization $\tilde{V}_{p \sigma}(t)=V_{p \sigma}(t) \exp \left[\int_{0}^{t} d \bar{t}\left(\epsilon_{p \sigma}(\bar{t})-\epsilon_{p \sigma}(0)\right)\right]$. For time-independent bath energies, Eq. 3.5.5 reads

$$
g_{p \sigma}\left(t, t^{\prime}\right)=i\left[f\left(\epsilon_{p \sigma}-\mu\right)-\Theta_{\mathcal{C}}\left(t, t^{\prime}\right)\right] e^{i\left(\epsilon_{p \sigma}-\mu\right)\left(t-t^{\prime}\right)} .
$$



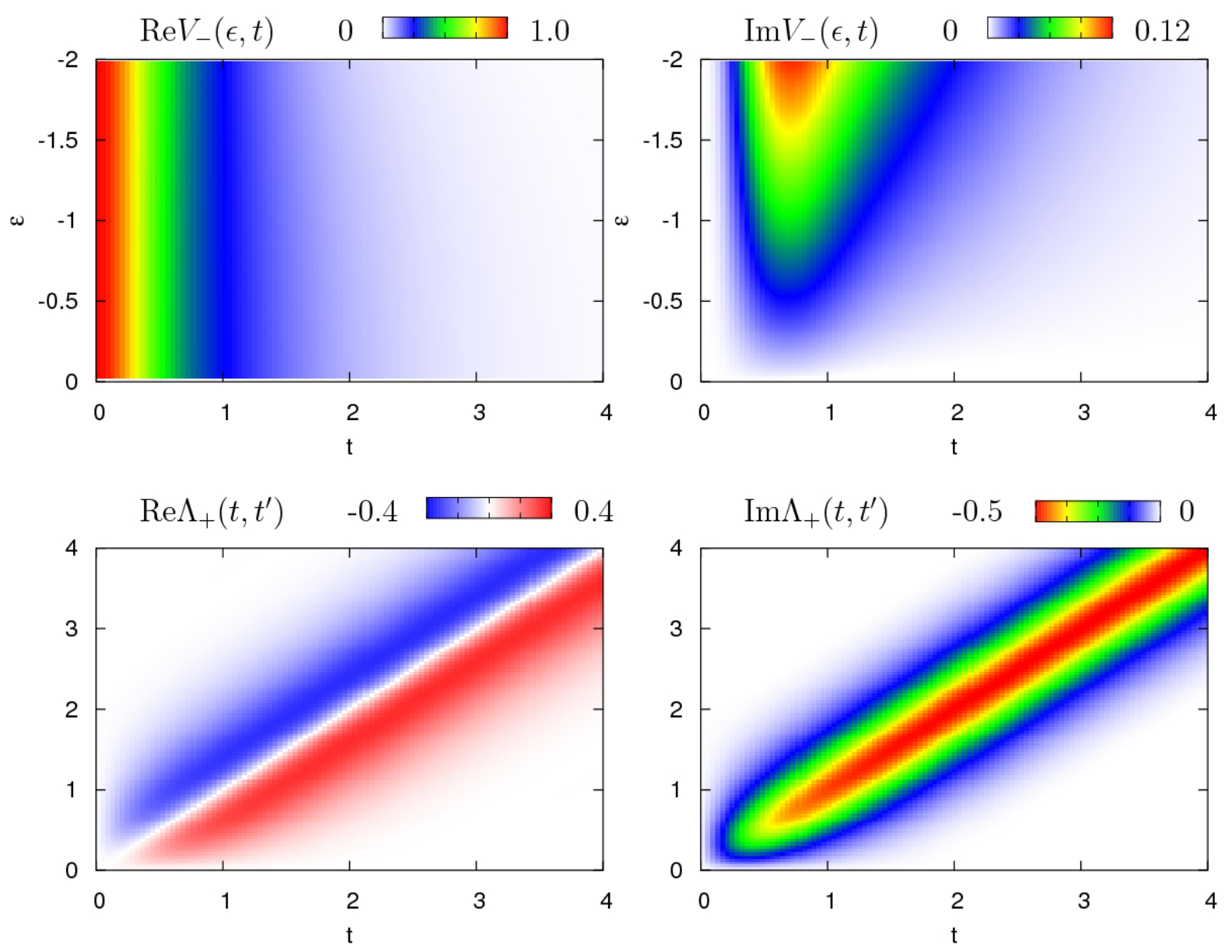

Figure 3.2: Illustration of the mapping of the single-site problem in DMFT to the SIAM (3.49). $\Lambda\left(t, t^{\prime}\right)$ is taken to be the SPT solution of an interaction quench in the Hubbard model to $U=5$, as in Fig. 3.1. The initial temperature is $T=0$. Upper panel: Hybridization $V_{-}(\epsilon, t)$ of the impurity with the "initial state" bath, Eqs. (3.63) and (3.60). $V_{-}(\epsilon, t)$ decays to 0 for $t \rightarrow \infty$. Lower panel: $\Lambda_{+}^{<}\left(t, t^{\prime}\right)$ [Eq. 3.69]. The function vanishes for $t=0$ and $t^{\prime}=0$, and becomes a function of $t-t^{\prime}$ for $t \rightarrow \infty$, when the state becomes stationary. 
Let us now assume that the bath states may be characterized by a continuous energy band with density of states $\rho_{-}(\epsilon)$ and hybridization $V_{-}(\epsilon, t)$. Using Eqs. (3.54) and (3.56), one has to determine $\rho_{-}(\epsilon)$ and $V_{-}(\epsilon, t)$ such that

$$
\Lambda\left(t, t^{\prime}\right) \equiv \Lambda_{-}\left(t, t^{\prime}\right)=i \int d \epsilon \rho_{-}(\epsilon) V_{-}(\epsilon, t)\left[f(\epsilon-\mu)-\Theta_{\mathcal{C}}\left(t, t^{\prime}\right)\right] e^{i(\epsilon-\mu)\left(t-t^{\prime}\right)} V_{-}^{*}(\epsilon, t)
$$

Here and in the following we assume symmetry with respect to the spin and hence omit the index $\sigma$. The subscript "-" will become clear below. We first attempt to find parameters such that Eq. (3.57) holds for the Matsubara component $\Lambda^{\mathrm{M}}\left(\tau, \tau^{\prime}\right)=i \lambda^{\mathrm{M}}\left(\tau-\tau^{\prime}\right)$. This requires

$$
\lambda^{\mathrm{M}}(\tau)=\int d \epsilon \rho_{-}(\epsilon)\left|V_{-}(\epsilon, 0)\right|^{2} e^{\left(\tau-\tau^{\prime}\right)(\epsilon-\mu)}[f(\epsilon-\mu)-\Theta(\tau)] .
$$

After Fourier transformation to Matsubara frequencies and analytic continuation to real frequencies we get

$$
-\frac{1}{\pi} \operatorname{Im} \lambda^{\mathrm{M}}(\epsilon+i 0)=\rho_{-}(\epsilon)\left|V_{-}(\epsilon, 0)\right|^{2} .
$$

At $t=0$, Eq. (3.49) is thus given by the impurity model that describes the initial equilibrium state in DMFT. Either the density of states or the hybridization can be chosen freely, e.g.,

$$
\rho_{-}(\epsilon)=-\frac{1}{\pi} \operatorname{Im} \lambda^{\mathrm{M}}(\epsilon+i 0), \quad V_{-}(\epsilon, 0)=1 .
$$

Next, Eq. 3.57 must be satisfied for the mixed component $\Lambda\urcorner(t, \tau)$,

$$
\Lambda\urcorner(t, \tau)=i \int d \epsilon \rho_{-}(\epsilon) V(\epsilon, t) f(\epsilon-\mu) e^{i(\epsilon-\mu)(t+i \tau} V(\epsilon, 0) .
$$

Here we have used that the Hamiltonian parameter $V(\epsilon, t)$ does not depend on imaginary time. After Fourier transformation to real frequencies (Sec. 2.2.3), we find

$$
\Lambda\urcorner(t, \epsilon+i 0)-\Lambda\urcorner(t, \epsilon-i 0)=\rho_{-}(\epsilon) e^{i \epsilon t} V_{-}(\epsilon, t) .
$$

Using Eq. (3.59) this gives

$$
\frac{V_{-}(\epsilon, t)}{V_{-}(\epsilon, 0)}=e^{i \epsilon t} \frac{\Lambda\urcorner(t, \epsilon+i 0)-\Lambda\urcorner(t, \epsilon-i 0)}{\operatorname{Im} \lambda^{\mathrm{M}}(\epsilon+i 0)} .
$$

For a nondegenerate energy band, the hybridization matrix elements of the time-dependent SIAM are thus uniquely determined already by $\Lambda\urcorner(t, \tau)$ and $\lambda^{\mathrm{M}}(\tau)$. This cannot be true in general: $\Lambda\urcorner(t, \tau)$ usually vanishes in the limit $t \rightarrow \infty$ (indicating that there are no correlations between the initial state and states at $t=\infty$ unless the system is in equilibrium), but the hybridization of the impurity site with the bath does not vanish in this limit. Otherwise the final state at $t=\infty$ would be trivial. For an illustration in a specific situation, cf. Fig. 3.2 . 
To resolve this puzzle it is sufficient to add a second band to the bath (subscript + ). In analogy to (3.57) we then have to satisfy

$$
\Lambda\left(t, t^{\prime}\right)=i \sum_{\alpha= \pm} \int d \epsilon \rho_{\alpha}(\epsilon) V_{\alpha}(\epsilon, t)\left[f(\epsilon-\mu)-\Theta_{\mathcal{C}}\left(t, t^{\prime}\right)\right] e^{i(\epsilon-\mu)\left(t-t^{\prime}\right)} V_{\alpha}^{*}(\epsilon, t) .
$$

In this expression we now fix

$$
V_{+}(\epsilon, 0)=0
$$

and determine $V_{-}(\epsilon, t)$ and $\rho_{-}(\epsilon)$ according to Eqs. (3.60) and (3.63). It then follows that the +-contribution to Eq. (3.64) vanishes for the Matsubara and mixed components of $\Lambda$, and hence Eq. (3.64) is satisfied for $\lambda^{\mathrm{M}}(\omega)$ and $\left.\Lambda\right\urcorner(t, \tau)$ by construction. Furthermore, because $\Lambda$ is continuous on $\left.\mathcal{C}[\Lambda\urcorner(\tau, t)=\Lambda^{<}(t, 0)\right]$, we have

$$
\Lambda^{<}(t, 0)=\Lambda_{-}^{<}(t, 0), \quad \Lambda^{<}(0, t)=\Lambda_{-}^{<}(0, t),
$$

where $\Lambda_{-}^{<}\left(t, t^{\prime}\right)$ is determined in terms of $\lambda^{\mathrm{M}}(\omega)$ and $\left.\Lambda\right\urcorner(t, \tau)$ by Eqs. (3.57), 3.60), and (3.63)

$$
\Lambda_{-}^{<}\left(t, t^{\prime}\right)=-\frac{i}{\pi} \int_{-\infty}^{0} d \epsilon f(\epsilon-\mu) \frac{\left.\left.\left.\left.[\Lambda\urcorner\left(t, \epsilon^{+}\right)-\Lambda\right\urcorner\left(t, \epsilon^{-}\right)\right][\Lambda\urcorner\left(t^{\prime}, \epsilon^{+}\right)^{*}-\Lambda\right\urcorner\left(t^{\prime}, \epsilon^{-}\right)^{*}\right]}{\operatorname{Im} \lambda^{\mathrm{M}}\left(\epsilon^{+}\right)},
$$

using the notation $\epsilon^{ \pm}=\epsilon \pm i 0$.

It thus remains to find $V_{+}(\epsilon, t)$ and $\rho_{+}(\epsilon)$ such that (3.64) also holds for the real-time components $\Lambda^{<}\left(t, t^{\prime}\right)$ and $\Lambda^{>}\left(t, t^{\prime}\right)$. In the following we will show that this is possible, and suggest a procedure to construct $V(\epsilon, t)$ in practice. We restrict the discussion to (initial state) temperature $T=0$ and particle-hole symmetric Green functions $(\mu=0)$, i.e., we assume $\Lambda^{<}\left(t, t^{\prime}\right)=\Lambda^{>}\left(t, t^{\prime}\right)^{*}$. Due to particle-hole symmetry, Eq. (3.64) holds for $\Lambda^{>}\left(t, t^{\prime}\right)$, provided that it holds for $\Lambda^{<}\left(t, t^{\prime}\right)$ and the hybridization satisfies

$$
V_{ \pm}(\epsilon, t)=V_{ \pm}(t,-\epsilon)^{*}
$$

For $T=0$ we have $f(\epsilon-\mu)=\Theta(-\epsilon)$. It is thus sufficient to determine $V_{+}(\epsilon, t)$ and $\rho_{+}(\epsilon)$ for $\epsilon<0$, such that Eq. (3.64) holds for $\Lambda^{<}\left(t, t^{\prime}\right)$, i.e.,

$$
\begin{aligned}
\Lambda_{+}^{<}\left(t, t^{\prime}\right) & =\Lambda^{<}\left(t, t^{\prime}\right)-\Lambda_{-}^{<}\left(t, t^{\prime}\right) \\
& =i \int_{-\infty}^{0} d \epsilon \rho_{+}(\epsilon) V_{+}(\epsilon, t) e^{i \epsilon\left(t-t^{\prime}\right)} V_{+}\left(\epsilon, t^{\prime}\right)^{*} .
\end{aligned}
$$

From Eq. (3.67) and the symmetry 2.15b) it follows that

$$
\Lambda_{+}^{<}\left(t, t^{\prime}\right)=-\Lambda_{+}^{<}\left(t^{\prime}, t\right)^{*}
$$

and hence $i \Lambda_{+}^{<}\left(t, t^{\prime}\right)$ is a Hermitian matrix in the time arguments. Eq. (3.70) is the continuous analog to the decomposition $A=L d L^{\dagger}$ of a matrix into a diagonal part $d$ and 
a square matrix $L$. Such a decomposition does indeed exist for Hermitian matrices $A$, e.g., the Choleski decomposition (where $L$ is upper triangular) or the eigen-decomposition (where $L$ is unitary). This indicates that Eq. (3.64) can be satisfied for an arbitrary choice of $\Lambda$ by a suitable choice of $V_{+}(\epsilon, t)$.

Practically, however, going from the discrete matrix decomposion to the continuous one [Eq. (3.70] ] is not straightforward. One possibility is to try the following: Introduce a discretization $\Delta t$ of time and $\Delta \epsilon$ of energy, define matrices $V_{j n}=V(-n \Delta \epsilon, j \Delta t)$ and $\Lambda_{j l}=\Lambda_{+}^{<}(j \Delta t, l \Delta t)$, discretize the integral in 3.70 ,

$$
\Lambda_{i j}=\Delta \epsilon \sum_{n} V_{i n} \rho_{n} V_{n l}^{\dagger}
$$

and perform a Cholesky or eigen-decomposition of $\Lambda_{i j}$ to determine $V_{i n}$ and $\rho_{n}$. However, this scheme is of little use, because then the matrix $V_{j n}=V(-n \Delta \epsilon, j \Delta t)$ does generally not converge to a continuous function of $\epsilon$ and $t$ for $\Delta t \rightarrow 0$ and $\Delta \epsilon \rightarrow 0$. On the contrary, the decomposition $(3.72)$ is not unique, because an arbitrary unitary matrix $U$ with $[U, \rho]$ $=0$ can be inserted, $V \rho V^{\dagger}=(V U) \rho(V U)^{\dagger}$ (since $\rho$ is diagonal, $[U, \rho]=0$ holds when $U$ performs column permutations multication of columns with arbitrary pure phase factors). Continuity of $V(\epsilon, t)$ is needed if the impurity Hamiltonian is to be solved numerically, and it requires a particular choice of $U$.

To overcome this problem one could impose a constraint on $V_{\text {in }}$ in (3.72), and solve the equation using maximum entropy techniques. We suggest a different approach, which introduces the discretization of $t$ and $\epsilon$ at a later stage and requires only the solution of linear equations $4^{4}$ The key idea of this approach is to start from a function $A_{0}\left(t, t^{\prime}\right)$ whose decomposition in terms of continuous functions $V_{0}(t, \epsilon)$ is known

$$
A_{0}\left(t, t^{\prime}\right)=\int_{-\infty}^{0} d \epsilon V_{0}(t, \epsilon) V_{0}\left(t^{\prime}, \epsilon\right)^{*}
$$

We then define

$$
\begin{aligned}
B\left(t, t^{\prime}\right) & =i \Lambda_{+}^{<}\left(t, t^{\prime}\right)-A_{0}\left(t, t^{\prime}\right) \\
A\left(t, t^{\prime} ; x\right) & =A_{0}\left(t, t^{\prime}\right)+x B\left(t, t^{\prime}\right),
\end{aligned}
$$

such that $A\left(t, t^{\prime} ; 0\right)=A_{0}\left(t, t^{\prime}\right)$ and $A\left(t, t^{\prime} ; 1\right)=i \Lambda_{+}^{<}\left(t, t^{\prime}\right)$. One can then attempt to continuously deform the known $V_{0}(t, \epsilon)$ into the unknown $V_{+}(t, \epsilon)$, by computing

$$
A\left(t, t^{\prime} ; x\right)=\int_{-\infty}^{0} d \epsilon V(t, \epsilon ; x) V\left(t^{\prime}, \epsilon ; x\right)^{*}
$$

for $x \in[0,1]$, choosing a constant $\rho_{+}(\epsilon)$. For this we take the derivative of Eq. (3.76) with respect to $x$,

$$
B\left(t, t^{\prime}\right)=\int_{-\infty}^{0} d \epsilon\left[\partial_{x} V(t, \epsilon ; x)\right] V\left(t^{\prime}, \epsilon ; x\right)^{*}+\int_{-\infty}^{0} d \epsilon V(t, \epsilon ; x)\left[\partial_{x} V\left(t^{\prime}, \epsilon ; x\right)^{*}\right] .
$$

\footnotetext{
${ }^{4}$ In contrast, solving Eq. 3.72 essentially corresponds to the determination of the square root of $\Lambda$.
} 
The solution of this equation for $\partial_{x} V(t, \epsilon ; x)$ is still not unique, but the arbitrariness can be fixed in a natural way: We choose some matrix $C$ with $B\left(t, t^{\prime}\right)=C\left(t, t^{\prime}\right)+C\left(t^{\prime}, t\right)^{*}$, e.g., $C=\frac{1}{2} B$, and require

$$
C\left(t, t^{\prime} ; x\right)=\int_{-\infty}^{0} d \epsilon\left[\partial_{x} V(t, \epsilon ; x)\right] V\left(t^{\prime}, \epsilon ; x\right)^{*} .
$$

This equation is a linear equation for $\partial_{x} V(t, \epsilon ; x)$ in terms of $V(t, \epsilon ; x)$, i.e., a linear differential equation for $V(t, \epsilon ; x)$. This differential equation must be solved for $x \in[0,1]$ with initial condition $V(t, \epsilon ; 0)=V_{0}(t, \epsilon)$ in order to obtain a continuous function

$$
V_{+}(t, \epsilon)=V(t, \epsilon ; 1)
$$

for which Eq. (3.69) is then satisfied. Whether this method work in practice, and whether it is superior to the maximum entropy techniques mentioned above, is beyond the scope of this thesis and should be clarified in the future. 


\section{Part II}

Time-evolution of simple model systems 


\section{Chapter 4}

\section{Approach to the thermal state}

\subsection{Introduction}

From everyday experience we know that any physical system will quickly approach an equilibrium state when it is left alone after a perturbation. The paradigm of this equilibration is the mixing of two gases or liquids. Turbulence and diffusion eventually lead to a homogeneous distribution of the two components, which has no memory of the initial preparation of the mixture. Without this rapid loss of memory, thermodynamics would make little sense. While the properties of an equilibrium state are highly universal and depend only on few parameters such as the energy per volume, the description of a nonequilibrium system requires much more detailed knowledge about the state. On the other hand, the loss of memory implies the irreversibility of thermodynamics which at first glance seems to be at odds with the deterministic mechanical laws that govern the motion of the atoms. This discrepancy is still not completely resolved more than one century after statistical physics was introduced mainly by Maxwell, Boltzmann, and Gibbs in order to put the macroscopic thermodynamic laws on a microscopic basis. In statistical physics, equilibrium properties of a large system are obtained by averaging over all accessible microscopic states [106]. For an isolated system, which can neither exchange energy nor particles with the environment, the possible states are those that satisfy the conservation of energy and particle number. By construction, such an average results in universal behavior. Furthermore, for large system sizes $L$, the statistical fluctuations of extensive observables about the average become small compared to the mean (the relative deviation is proportional to $1 / \sqrt{L}$ ), i.e., a randomly chosen, or "typical" accessible state displays the same physical properties as the equilibrium state.

However, a nonequilibrium state is in general not typical in the above sense, and it is thus not immediately clear why the statistical description usually works well for arbitrary initial conditions. Boltzmann and Gibbs introduced the concepts of ergodic and mixing dynamics to show that it is the chaotic motion of the atoms which justifies the use of statistical mechanics, irrespective of the initial state 1 Both concept involve some averaging, which is however more moderate than the microcanonical average over

\footnotetext{
${ }^{1}$ Introductory texts on these issues can be found, e.g., in the books by Dorfman [107] and Gutzwiller [108].
} 
all accessible states. Ergodicity ensures that the time average of an observable equals the microcanonical average for almost all initial states, while Gibbs proposed that mechanical systems are mixing, which asserts that averages over an arbitrary small uncertainty in the initial state at infinite past can be replaced by the microcanonical ensemble. Neither ergodic or mixing dynamics requires any randomness in the equations of motion, which are deterministic and include no coupling to the environment $2^{2}$ Unfortunately, it has turned out extremely hard to prove either of these properties even for the simplest classical mechanical systems [107].

The following three chapters explicitly deal with the relation of the statistical behavior of large systems to their quantum mechanical properties. While Ch. 5 and 6 focus on specific model systems, we discuss some fundamental questions in more general language in the present chapter. Does a system really equilibrate for an arbitrary initial state, and if it does, does the final state equal the thermal state? Some preliminary remarks and well-known concepts are presented in Sec. 4.2. When the system is not ergodic, it can still reach a stationary state, which however retains detailed memory on the initial state. In Sec. 4.3 we discuss a possible statistical description of that state. Furthermore we address the important question of how the relaxation proceeds in time (Sec. 4.4). It turns out that the state can become trapped in a nonthermal quasistationary state for long times, in particular for systems which are close to integrability (Sec. 4.5).

One should stress that the concepts introduced in the following are not intended to "justify statistical physics" by showing that macroscopic laws can be derived from the microscopic ones. Actually, statistical physics can be motivated without reference to the mechanical properties of the system, from a purely information theoretical point of view [109] (Sec. 4.3). In contrast, the concepts discussed in the following should give an understanding of the real-time relaxation behavior of many-particle systems which is observable in experiment in spite of the very short relaxation times that are usually involved. Some experiments are discussed in Sec. 4.2 for cold atomic gases, and in Ch. 8 for solid state systems. The relevance for experiment is particularly evident when ergodicity fails completely, or when thermalization is prevented on timescales which are much longer that those estimated from the bare energy scales of the system.

\subsection{Thermalization in classical and quantum systems}

\section{Classical mechanics}

Although the subject of this thesis are quantum mechanical systems, the classical concepts will be shortly introduced here for later comparison to their quantum analogues. As mentioned in the previous section, thermalization in classical systems is attributed to their chaotic dynamics, which is substantiated through the concept of ergodicity [107, 108] due to Boltzmann: A Hamiltonian system is ergodic if a generic trajectory in phase space densely covers the whole surface of constant energy, such that the time spent by the

\footnotetext{
${ }^{2}$ On the other hand, effective equations of motion for few-particle observables such as the momentum distribution (the Boltzmann equation) are irreversible.
} 

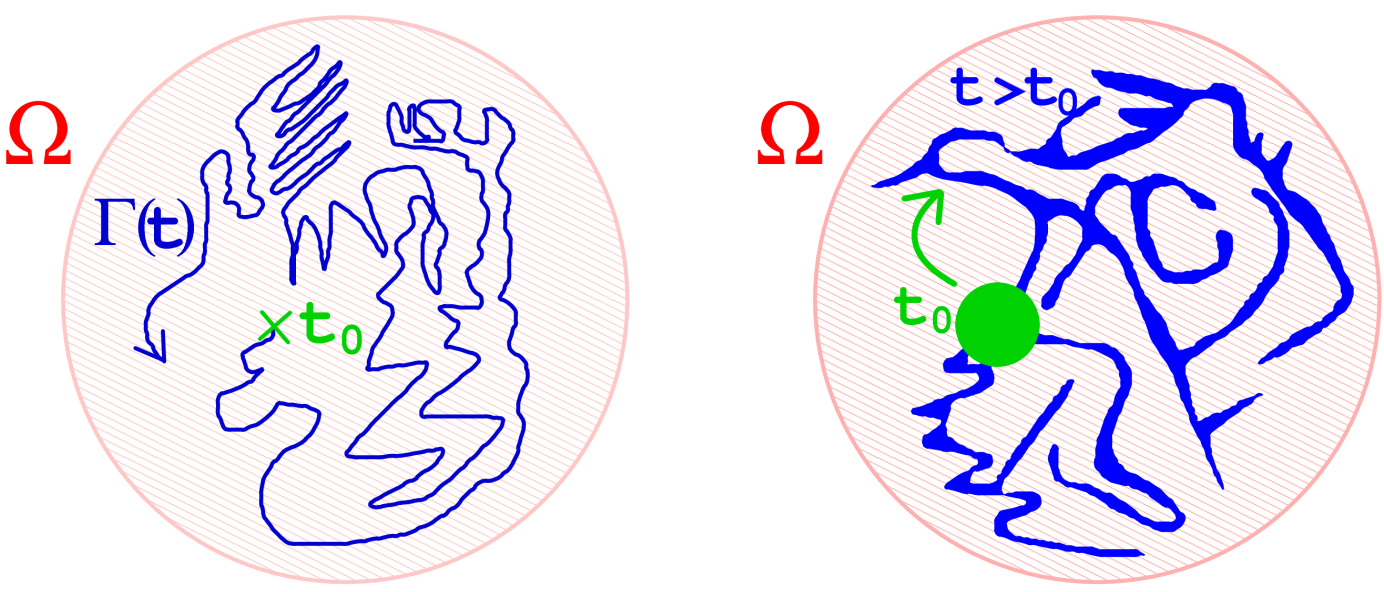

Figure 4.1: Illustration of ergodic and mixing behavior in phase space $\Omega$. Left panel: For an ergodic system, a trajectory which starts from a generic point at time $t=t_{0}$ densely covers phase space. Right panel: The unitary time evolution of a mixing system deforms a generic set of initial states at time $t=t_{0}$ such that it becomes dense in phase space for $t \rightarrow \infty$.

trajectory in any open set is proportional to the phase-space volume of this set (Fig. 4.1). From this geometrical property it follows that the time average $\overline{\langle O\rangle}$ of any observable $O(\boldsymbol{q}, \boldsymbol{p})$ which is a continuous function of the coordinates $\boldsymbol{q}$ and momenta $\boldsymbol{p}$ equals the microcanonical average $\langle O\rangle_{\text {mic }}$ over the surface of constant energy,

$$
\overline{\langle O\rangle} \equiv \lim _{T \rightarrow \infty} \frac{1}{T} \int_{0}^{T} d t O(\boldsymbol{q}(t), \boldsymbol{p}(t))=\frac{\int_{\Omega} d \boldsymbol{p} d \boldsymbol{q} \delta(E-H) O(\boldsymbol{q}, \boldsymbol{p})}{\int_{\Omega} d \boldsymbol{p} d \boldsymbol{q} \delta(E-H)} \equiv\langle O\rangle_{\mathrm{mic}} .
$$

Here $H=H(\boldsymbol{q}, \boldsymbol{p})$ is the Hamiltonian, and $E=H(\boldsymbol{q}(0), \boldsymbol{p}(0))$ the energy on the trajectory $(\boldsymbol{q}(t), \boldsymbol{p}(t))$. A further characterization of the chaotic behavior was introduced by Gibbs through the notion of mixing dynamics (which is a stronger criterion than ergodicity). It implies that any small set in phase space is dispersed over the whole phase space during the time evolution, while its volume is constant due to Liouville's theorem, such that the average over initial states can be replaced by the microcanonical average. Rigorous proofs for ergodicity exits only in rather simple systems such as the classical gas of hard spheres [110], or so-called billiard systems [111], where few hard-core particles are caged in a complex geometry and reflected elastically from the walls. On the other hand, a system does not behave ergodic when it is integrable. A classically integrable system with $N$ degrees of freedom is defined to have $N$ constants of motion $\mathcal{I}_{k}(\boldsymbol{q}, \boldsymbol{p})$ that are smooth functions on phase space, and all pairwise Poisson brackets vanish, $\left\{\mathcal{I}_{k}, \mathcal{I}_{l}\right\}=0$. The phase space of an integrable system is foliated by $N$-dimensional smooth submanifolds, the invariant tori, that are defined by fixing all constants of motion to given values. Because the orbits are confined to those invariant tori and a smooth submanifold cannot be dense in phase space, the system cannot behave ergodic.

Due to the lack of analytical statements, Fermi, Pasta, and Ulam made an attempt to demonstrate ergodic behavior by the numerical simulation of a finite chain of an- 
harmonically coupled oscillators $3^{3}$ Their calculation, which was actually one of the first large-scale numerical calculation of civil purpose, led to a surprise which became known as the Fermi-Pasta-Ulam (FPU) paradox: Even at the largest accessible times thermal equilibrium, marked by equipartition of the energy among the normal modes, was not reached. Instead, the system showed almost perfect recurrences to the initial state. The FPU paradox remains an active area of research even after 50 years (for reviews see Refs. [113, 114]), and its investigation has led to the discovery of fundamentally new phenomena such as soliton waves [115]. A possible resolution of the FPU paradox relates it to the fact that the Hamiltonian is close to integrability [113]. The transition from nonergodic to chaotic behavior occurs at some precise threshold in the excitation energy, which depends on the degree of anharmonicity and on the system size [113, 116], and seems to vanish in the thermodynamic limit [117]. The dynamics of nearly integrable systems will be discussed below in Sec. 4.5 .

\section{Quantum mechanics: experiments and general remarks}

For quantum-mechanical systems, the fundamental questions are similar as for classical systems, although the time evolution of a pure initial state $|\Psi(0)\rangle$ of a closed system is governed by the linear Schrödinger equation with Hamiltonian $H$,

$$
i \partial_{t}|\Psi(t)\rangle=H(t)|\Psi(t)\rangle
$$

The time evolution of the state $|\Psi(t)\rangle$ is then unitary, such that the system remains in a pure state if it is initially in a pure state. The wave function an isolated system does not converge at all for $t \rightarrow \infty$, in particular not to a mixed state with density matrix $\operatorname{Tr} \rho^{2}<1$. Only the reduced density matrix of a finite subsystem, for which the rest of the system effectively acts as a reservoir, can become stationary [118]. Nevertheless, the entire system can relax in the weaker sense that expectation value $\langle\Psi(t)|O| \Psi(t)\rangle$ of observables $O$ becomes stationary for large times. This global relaxation can happen only for sufficiently large systems to prevent finite recurrence times, and for sufficiently simple observables. ${ }^{4}$ For example, the expectation value of an observable $O=\left|n_{1}\right\rangle\left\langle n_{1}|+| n_{2}\right\rangle\left\langle n_{2}\right|$ that is the linear combination of projectors onto two eigenstates of $H$ with different energies, oscillates for all times. However, such projectors are highly nonlocal and their expectation values correspond to correlation functions of very high order, while relaxation of expectation values is expected for local and few-particle observables. However, there are some cases where relaxation is prevented because only some frequencies occur in the time evolution, which can happen in very simple systems such as the Ising chain in a transverse magnetic field [119], or the atomic limit of the Hubbard model (see below).

The interest in thermalization of quantum-mechanical systems was recently boosted by experiments on ultracold atomic gases [12] that can directly address this issue [120, 121].

\footnotetext{
${ }^{3}$ Fermi died in 1954 before the work was published, and the paper appeared only as a preprint 112 .

${ }^{4}$ The fact that statements on relaxation and thermalization only hold for a certain class of observables is of course not specific to quantum mechanics. However, the definition of this class is easier in the classical case. For example, Eq. 4.1 holds for observables that are continuous functions on phase space.
} 
For example, Kinoshita, Wenger, and Weiss realized a system of many interacting particles that does not relax to thermal equilibrium, but to a non-thermal stationary state instead. For this purpose, a one-dimensional gas of cold $\mathrm{Rb}$ atoms was initially prepared such that two parts of the atom-cloud move in opposite directions. While the two clouds oscillate against each other in the trap, the atoms undergo many transitions, and the momentum distribution finally becomes stationary $5^{5}$ However, this stationary distribution still has detailed memory on the initial state, and hence the system does not thermalize on the accessible times. This behavior has been attributed to the fact that the particular system resembles hard-core bosons, which are integrable in one dimension [120, 122, 123]. In another experiment, a Bose condensate was prepared in the potential of an optical lattice which was suddenly increased [16]. The Hamiltonian is then essentially reduced to the Hubbard interaction $H=U \sum_{i} n_{i}\left(n_{i}-1\right)$, but no hopping between lattice sites occurs. Because $H / U$ has only integer eigenvalues, the wave function will oscillate for all times with period $2 \pi \hbar / U$ in this case. These oscillations have been termed collapse and revival oscillations, because they are manifest in the periodic destruction and reformation of the coherent $\boldsymbol{k}=0$ peak in the momentum distribution (which indicates the presence of the Bose Einstein condensate). These recent experiments have motivated theoretical investigations of quenches in various models. These include the Hubbard model for bosons [124, 125] and fermions [126, 127, 128, 74], the related Falicov-Kimball model [71], hardcore bosons in one and two dimensions [122, 123, 129, 130], the integrable Luttinger model [131, 132], spinless fermions [133, 134], and Heisenberg spin chains [135].

\section{Long-time average and the diagonal ensemble}

In order to address the issue of thermalization we need to determine the long-time limit of a quantum system for an arbitrary initial state. For this purpose consider an isolated system is prepared at time $t=0$ in an initial state which is described by the density matrix $\rho_{0}$, while the time evolution for $t>0$ is governed by an arbitrary time-independent Hamiltonian $H$. In general $\rho_{0}=\sum_{n} p_{n}\left|\Psi_{n}\right\rangle\left\langle\Psi_{n}\right|$ is a statistical mixture of orthogonal states $\left|\Psi_{n}\right\rangle$ with probabilities $p_{n}$, but it has the form $\rho_{0}=|\Psi(0)\rangle\langle\Psi(0)|$ if the initial state is a puse state. For $t \geq 0$ the time evolution of the density matrix is given by

$$
\rho(t)=e^{i H t} \rho_{0} e^{-i H t}
$$

and the expectation value of an observable $O$ is

$$
\langle O(t)\rangle_{0}=\operatorname{Tr}[O \rho(t)]=\sum_{n n^{\prime} g g^{\prime}} e^{-i\left(E_{n}-E_{n^{\prime}}\right) t}\left\langle n g|O| n^{\prime} g^{\prime}\right\rangle\left\langle n^{\prime} g^{\prime}\left|\rho_{0}\right| n g\right\rangle,
$$

where $|n g\rangle$ are the eigenstates of $H$ with energies $E_{n}$, and $g$ labels possible degeneracies. If the long-time $\operatorname{limit}_{\lim _{t \rightarrow \infty}}\langle O(t)\rangle_{0}$ exists, then it is necessarily equal to the long-time

\footnotetext{
${ }^{5}$ The experiment is not quite as simple as suggested here: The stationary distribution is only observed after a subtle correction for extrinsic loss effects.
} 
average $\overline{\langle O\rangle}$,

$$
\overline{\langle O\rangle}=\lim _{T \rightarrow \infty} \frac{1}{T} \int_{0}^{T} d t\langle O\rangle_{t}=\sum_{n g g^{\prime}}\left\langle n g|O| n g^{\prime}\right\rangle\left\langle n g^{\prime}\left|\rho_{0}\right| n g\right\rangle,
$$

Here we assume that the limit can be taken termwise. In a steady state the system is thus described by the density matrix

$$
\rho_{\text {diag }}=\sum_{n g g^{\prime}}|n g\rangle\left\langle n g\left|\rho_{0}\right| n g^{\prime}\right\rangle\left\langle n g^{\prime}\right|,
$$

which has been termed the time-averaged density matrix [125] or the diagonal ensemble [129], and has been discussed since the early days of quantum mechanics [136]. The statistical operator $\rho_{\text {diag }}$ itself is extremely complicated and of little practical use. However, it can be used as a starting point to discuss much simpler, statistical descriptions of the stationary state in nonintegrable systems (next paragraph) and integrable systems [126] (Sec. 4.3).

Is the prediction of $\rho_{\text {diag }}$ equal to the microcanonical or canonical ensemble? In the following we will call a quantum system ergodic if this is the case. From Eq. 4.5 one may infer that quantum systems are not ergodic unless the matrix elements are given by $\left\langle n g\left|\rho_{0}\right| n g^{\prime}\right\rangle \propto \delta_{g g^{\prime}} \Theta\left(\left|E_{n}-E\right|-\Delta E\right) / \Delta E$ (microcanonical ensemble) or $\left\langle n g\left|\rho_{0}\right| n g^{\prime}\right\rangle \propto$ $\delta_{g g^{\prime}} e^{-\beta E_{n}}$ (canonical ensemble), which is unlikely for an arbitrary initial state. However, these matrix elements, or equivalently, the energy distribution, is not a good indicator for nonergodic behavior. In fact, the energy distribution $P(E) \propto \sum_{n g g^{\prime}}\left\langle n g\left|\rho_{0}\right| n g^{\prime}\right\rangle \delta\left(E-E_{n}\right)$ is different between the microcanonical and canonical ensemble, but nevertheless both ensembles give the same value for other ensembles in the thermodynamic limit. Rather, ergodic behavior can be related to the diagonal matrix elements $\left\langle n g|O| n g^{\prime}\right\rangle$ of observables. This is the main content of the following eigenstate thermalization hypothesis [137, 138, which states that

the expectation value of a large class of observables in a single energy eigenstate

$|n\rangle$, can be replaced by the microcanonical average, $\langle n|O| n\rangle=\langle O\rangle_{m i c}\left(E_{n}\right)$.

A similar property has been discussed under the name "typicality" [139]. The corresponding concept for open quantum systems, "canonical typicality" [140, 141], means that each energy eigenstate of system and environment reduces to the canonical density matrix of the system alone when the environment is traced out. When the eigenstate thermalization hypothesis (4.7) holds, the long-time average (4.5) yields the microcanonical average provided that $P(E)$ is sharply peaked, and degeneracies do not play a role. The precise form of $P(E)$, however, does no longer matter. A proof of (4.7) exists only under strong assumptions, e.g., within a semiclassical treatment of classically chaotic systems provided that Berry's conjecture can be applied [142, 138]. Rigol et al. [129] demonstated the validity of the eigenstate thermalization hypothesis in a finite system using exact diagonalization. We will come back to this issue in Sec. 4.5, where the breakdown of 4.7) is discussed as the Hamiltonian comes close to integrability. 


\section{The effective temperature}

If a system thermalizes, all expectation values in the final state are determined by the conserved energy $E$ and particle number $N$. For any system in nonequilibrium (with a time-independent, number conserving Hamiltonian $H$ ) we can thus introduce the notion of an effective temperature $T_{*}$ and effective chemical potential $\mu_{*}$, such that the grandcanonical ensemble $\rho_{*}=e^{-\left(H-\mu_{*} N\right) / T_{*}} / Z$ has the same average energy $E=\operatorname{Tr}\left[\rho_{0} H\right]$ and particle number as the original system, i.e.,

$$
\begin{aligned}
& \operatorname{Tr}\left[e^{-\left(H-\mu_{*} N\right) / T_{*}} H\right] / Z=\operatorname{Tr}\left[\rho_{0} H\right] \\
& \operatorname{Tr}\left[e^{-\left(H-\mu_{*} N\right) / T_{*}} N\right] / Z=\operatorname{Tr}\left[\rho_{0} N\right],
\end{aligned}
$$

with $Z=\operatorname{Tr}\left[e^{-\left(H-\mu_{*} N\right) / T_{*}}\right]$. Those equations fix $T_{*}$ and $\mu_{*}$ in terms of inital state and equilibrium quantities. Thermalization of a system in the thermodynamic limit is then equivalent to the fact that long-time limits of simple observables $O$ equals the avarage in the grand canonical ensemble, $\lim _{t \rightarrow \infty}\langle O(t)\rangle_{0}=\operatorname{Tr}\left[e^{-\left(H-\mu_{*} N\right) / T_{*}} O\right] / Z$. From the second law of thermodynamics one expects that the entropy of the grand-canonical ensemble is higher than the entropy of the initial state. However $T_{*}$ may be negative in principle, which would mean that an initial state thermalizes to state with population inversion. This was however not the case for the systems studied in the present thesis.

\subsection{Statistical predictions for integrable systems}

\section{Generalized Gibbs ensemble}

It was discussed already in the previous sections that a classical integrable system cannot thermalize because its motion is constrained by conservation laws in addition to the energy-conservation. The situation is similar for a quantum mechanical systems. Simple theoretical models that do not thermalize have been studied for a long time [143, 144, 145, 146, 147]. Motivated by cold atom experiments the question of nonergodic behavior of quantum mechanical systems has been taken up by several authors (see below). Rigol et al. [123] investigated a system of hard-core bosons on a one-dimensional lattice that are initially confined to a finite region of space, which is suddenly expanded at time $t=$ 0 . The momentum distribution relaxes to a stationary value, but this is not equal to the thermal distribution and instead carries detailed memory on the initial state. The reason for this behavior lies in the integrable nature of the system: There is an exact mapping of the one-dimensional gas of hard-core bosons to noninteracting fermions by means of the Jordan-Wigner transformation, and it is clear that the momentum distribution $n_{\boldsymbol{k}}$ of the effective fermions cannot relax because in a noninteracting system the momentum of each single particle is conserved.

Because the memory of the system of its initial configuration must be somehow encoded in the values of these integrals of motion, Rigol et al. suggested to construct a density matrix $\rho_{\mathrm{G}}$ for the final stationary state only from that information [123]. Suppose a system has a number of conserved quantities $\mathcal{I}_{\alpha}$ that commute with each other and with 
the Hamiltonian, and whose expectation value $\left\langle\mathcal{I}_{\alpha}\right\rangle_{0}$ in known in the initial state. Then, according to information theory the most likely $\rho_{\mathrm{G}}$ maximizes the entropy $\operatorname{Tr}\left[\rho_{\mathrm{G}} \log \rho_{\mathrm{G}}\right]$ under the constraints $\operatorname{Tr}\left[\rho_{\mathrm{G}} \mathcal{I}_{\alpha}\right]=\left\langle\mathcal{I}_{\alpha}\right\rangle_{0}$. This argument was originally posed by Janes [109] to justify the use of statistical physics without making any reference to mechanical concepts like ergodicity. The density matrix for the contrained system, the generalized Gibbs ensemble (GGE) is then given by

$$
\rho_{\mathrm{G}}=\frac{\exp \left(-\sum_{\alpha} \lambda_{\alpha} \mathcal{I}_{\alpha}\right)}{\operatorname{Tr}\left[\exp \left(-\sum_{\alpha} \lambda_{\alpha} \mathcal{I}_{\alpha}\right)\right]},
$$

where the Lagrange parameters $\lambda_{\alpha}$ are fixed by the conditions $\operatorname{Tr}\left[\rho_{\mathrm{G}} \mathcal{I}_{\alpha}\right]=\left\langle\mathcal{I}_{\alpha}\right\rangle_{0}$. The GGE turns out to be valid for various models like hard-core bosons [123, 122] in one dimension, for the integrable Luttinger model [131, 132], the Falicov-Kimball model (Ch. 5 , Ref [71]), and the 1/r-Hubbard chain [126] introduced by Gebhard and Ruckenstein [148]. Barthel and Schollwöck [149] recently showed that dephasing, i.e., the relaxation of the density matrix to the GGE, occurs for finite subsystems under certain mathematical conditions on the spectrum. However, even for hard-core bosons, at least some observables like the unit-cell averaged one-particle correlation function is not predicted correctly [122, 123], thus asking for general criteria for the validity of GGEs. Some rather general critaria, which are presented in the following subsection, have been derived [126] as part of this thesis.

\section{Validity of generalized Gibbs ensembles}

In order to determine if a statistical description of the final state is valid one can compare it to the diagonal ensemble (4.6). While this approach leads to few analytical results in case of nonintegrable systems, where the best statistical description is the microcanonical one, it does lead to some useful criteria for integrable systems, where more information enters the generalized statistical prediction (4.9) [126]. The central point for the validity is the choice of the integrals of motion $\mathcal{I}_{\alpha}$ that enter Eq. (4.9), i.e., the information that has to be taken into account to describe the stationary state.

For quantum systems, there is no unique choice of the constants of motion $\mathcal{I}_{k}$ as for classical integrable systems. In fact, the notion of integrability in quantum systems is generally more involved than in classical systems [150]. In particular, the mere existence of many conserved quantities does not imply integrability. In contrast, for any quantum system the number of linearly independent observables that commute among each other equals the dimension $D$ of the Hilbert space. They can be chosen, e.g., as the projectors $P_{\alpha}$ on energy-eigenstates $|\alpha\rangle$, or, for a nondegenerate system, all powers $H^{n}(n \leq D)$ of the Hamiltonian are independent. In a system with nondegenerate spectrum, it is easy to show that the Gibbs ensemble that is constructed from these projectors or the powers of the Hamiltonian exactly recovers the diagonal ensemble [151, 130] 6. However, taking

\footnotetext{
${ }^{6}$ Because the projectors satisfy $P_{\alpha}^{2}=P_{\alpha}$, one has $\rho_{\mathrm{G}}=\exp \left(\sum_{\alpha} \lambda_{\alpha} P_{\alpha}\right) / Z=Z^{-1} \prod_{\alpha}\left[1+P_{\alpha}\left(e^{-\lambda_{\alpha}}-1\right)\right]$; using $P_{\alpha} P_{\beta}=0$ for orthogonal states $\alpha$ and $\beta$, and $\sum_{\alpha} P_{\alpha}=1$, this gives $\rho_{\mathrm{G}}=Z^{-1} \sum_{\alpha} P_{\alpha} e^{-\lambda_{\alpha}}$. The constraint evaluates to $\operatorname{Tr} \rho_{\mathrm{G}} P_{\alpha}=e^{-\lambda_{\alpha}} / Z$, which must equal $\left\langle P_{\alpha}\right\rangle_{0}=\left\langle\alpha\left|\rho_{0}\right| \alpha\right\rangle$. Hence $\rho_{\mathrm{G}}$ equals the diagonal ensemble $\rho_{\mathrm{G}}=\sum_{\alpha} P_{\alpha}\left\langle\alpha\left|\rho_{0}\right| \alpha\right\rangle$.
} 
all powers of $H$, or all projectors into account contradicts the idea of statistical physics, which is to make predictions although detailed information on the state is disregarded. Hence the choice of the correct conserved quantities is central to the whole idea of the GGE.

In the following we consider only integrable systems that can be mapped onto effectively noninteracting systems. This requirement is stronger than integrability via the Bethe ansatz, but it holds for all cases to which the GGE has been applied so far. For such systems the occupation of the effective free single-particle states forms a natural set of constants of motion $\mathcal{I}_{\alpha}$, and it is intersting to see whether these constants of motion are sufficient for a statistical description of the final state, or whether further constants of motion such as their products $\mathcal{I}_{\alpha} \mathcal{I}_{\beta}$ are needed. The general effective Hamiltonian for those systems is given by

$$
H_{\mathrm{eff}}=\sum_{\alpha} \epsilon_{\alpha} \mathcal{I}_{\alpha}
$$

where either (a) the constants of motion $\mathcal{I}_{\alpha}$ have eigenvalues 0 and 1 and can thus be represented by fermions or hard-core bosons, $\mathcal{I}_{\alpha}=a_{\alpha}^{\dagger} a_{\alpha}$, with $\left[a_{\alpha}, a_{\beta}^{\dagger}\right]_{ \pm}=\delta_{\alpha \beta},\left(a_{\alpha}\right)^{2}=\left(a_{\alpha}^{\dagger}\right)^{2}$ $=0$; or $(\mathrm{b})$ the $\mathcal{I}_{\alpha}$ have the eigenvalues $0,1,2 \ldots$ and can be represented by bosons, $\mathcal{I}_{\alpha}=$ $b_{\alpha}^{\dagger} b_{\alpha}$, with $\left[b_{\alpha}, b_{\beta}^{\dagger}\right]=\delta_{\alpha \beta}$. Examples for case (a) are the effective Hamiltonians for hardcore bosons in one dimension [122, 123], free fermions with quenched disorder [71], and the $1 / r$ fermionic Hubbard chain, whereas case (b) applies to the Luttinger model [131]. Note that in those cases the operators $a_{\alpha}$ or $b_{\alpha}$ are not the original degrees of freedom, and in turn, the $\mathcal{I}_{\alpha}$ are usually rather complex many-particle operators when expressed in terms of the original particles. For the two cases the Lagrange multipliers $\lambda_{\alpha}$ in Eq. (4.9) are then given by (a) $\ln \left[\left\langle\mathcal{I}_{\alpha}\right\rangle_{0}^{-1}-1\right]$ and $(\mathrm{b}) \ln \left[\left\langle\mathcal{I}_{\alpha}\right\rangle_{0}^{-1}+1\right]$.

We now investigate whether the prediction of the GGE coincides with the prediction of the diagonal ensemble for two general types of observables: for case (a) the observable

$$
A=\sum_{\substack{\alpha_{1} \cdots \alpha_{m} \\ \beta_{1} \cdots \beta_{m}}} A_{\beta_{1} \cdots \beta_{m}}^{\alpha_{1} \cdots \alpha_{m}} a_{\alpha_{1}}^{\dagger} \cdots a_{\alpha_{m}}^{\dagger} a_{\beta_{m}} \cdots a_{\beta_{1}}
$$

is considered, while for case (b) also powers of the bosonic operators are allowed, and therefor (for $r_{i}, s_{j} \geq 1$ )

$$
B=\sum_{\substack{\alpha_{1} \cdots \alpha_{m}, r_{1} \cdots r_{m} \\ \beta_{1} \cdots \beta_{m}, s_{1} \cdots s_{m}}} B_{\beta_{1} \cdots \beta_{m}, s_{1} \cdots s_{m}}^{\alpha_{1} \cdots \alpha_{m}, r_{1} \cdots r_{m}}\left(b_{\alpha_{1}}^{\dagger}\right)^{r_{1}} \cdots\left(b_{\alpha_{m}}^{\dagger}\right)^{r_{m}}\left(b_{\beta_{m}}\right)^{s_{m}} \cdots\left(b_{\beta_{1}}\right)^{s_{1}}
$$

is considered. Without loss of generality $B_{\beta_{1} \cdots \beta_{m}, s_{1} \cdots s_{m}}^{\alpha_{1} \cdots \alpha_{m}, r_{1} \cdots r_{m}}$ are assumed to vanish whenever two indices $\alpha_{i}$ or $\beta_{j}$ are the same.

The Hamiltonian (4.10) has the eigenstates $|\boldsymbol{m}\rangle$ with occupation numbers $\mathcal{I}_{\alpha}|\boldsymbol{m}\rangle=$ $m_{\alpha}|\boldsymbol{m}\rangle$ and energy eigenvalues $E_{\boldsymbol{m}}=\sum_{\alpha} \epsilon_{\alpha} m_{\alpha}$. For simplicity it is assumed that the degeneracy of energy eigenvalues is irrelevant, i.e., the observable $O$ or the initial-state 
density matrix $\rho_{0}$ are diagonal in the subspace of eigenvectors $|\boldsymbol{m}\rangle$ with the same energy. It is straightforward to obtain the long-time average, which is given by

$$
\overline{\langle O\rangle}=\sum_{\boldsymbol{m}}\langle\boldsymbol{m}|O| \boldsymbol{m}\rangle\left\langle\boldsymbol{m}\left|\rho_{0}\right| \boldsymbol{m}\right\rangle
$$

as well as the GGE averages (4.9) of the observables $A$ and $B$ by using the occupation number basis $|\boldsymbol{m}\rangle$ and the fixed GGE averages $\left\langle\mathcal{I}_{\alpha}\right\rangle_{\mathrm{G}}=\left\langle\mathcal{I}_{\alpha}\right\rangle_{0}$. In case (a) one finds find

$$
\begin{aligned}
\overline{\langle A\rangle} & =\sum_{\alpha_{1} \cdots \alpha_{m}} \widetilde{A}_{\alpha_{1} \cdots \alpha_{m}}\left\langle\prod_{i=1}^{m} \mathcal{I}_{\alpha_{i}}\right\rangle_{0}, \\
\langle A\rangle_{\mathrm{G}} & =\sum_{\alpha_{1} \cdots \alpha_{m}} \widetilde{A}_{\alpha_{1} \cdots \alpha_{m}} \prod_{i=1}^{m}\left\langle\mathcal{I}_{\alpha_{i}}\right\rangle_{0},
\end{aligned}
$$

where the identity

$$
\left\langle\prod_{i=1}^{m} \mathcal{I}_{\alpha_{i}}\right\rangle_{\mathrm{G}}=\prod_{i=1}^{m}\left\langle\mathcal{I}_{\alpha_{i}}\right\rangle_{\mathrm{G}}=\prod_{i=1}^{m}\left\langle\mathcal{I}_{\alpha_{i}}\right\rangle_{0}
$$

was used in the second line. In case (b) one finds

$$
\begin{aligned}
\overline{\langle B\rangle} & =\sum_{\alpha_{1} \cdots \alpha_{m}} \widetilde{B}_{\alpha_{1} \cdots \alpha_{m}}\left\langle\prod_{i=1}^{m}\left(b_{\alpha_{i}}^{\dagger}\right)^{r_{i}}\left(b_{\alpha_{i}}\right)^{r_{i}}\right\rangle_{0} \\
& =\sum_{\alpha_{1} \cdots \alpha_{m}} \widetilde{B}_{\alpha_{1} \cdots \alpha_{m}}\left\langle\prod_{i=1}^{m} \prod_{k=0}^{r_{i}-1}\left(\mathcal{I}_{\alpha_{i}}-k\right)\right\rangle_{0}, \\
\langle B\rangle_{\mathrm{G}} & =\sum_{\alpha_{1} \cdots \alpha_{m}} \widetilde{B}_{\alpha_{1} \cdots \alpha_{m}}\left\langle\prod_{i=1}^{m}\left(b_{\alpha_{i}}^{\dagger}\right)^{r_{i}}\left(b_{\alpha_{i}}\right)^{r_{i}}\right\rangle_{\mathrm{G}} \\
& =\sum_{\alpha_{1} \cdots \alpha_{m}} \widetilde{B}_{\alpha_{1} \cdots \alpha_{m}} \prod_{i=1}^{m}\left[r_{i} !\left(\left\langle\mathcal{I}_{\alpha_{i}}\right\rangle_{0}\right)^{r_{i}}\right],
\end{aligned}
$$

where the bosonic operator identity

$$
\left(b_{\alpha_{i}}^{\dagger}\right)^{r_{i}}\left(b_{\alpha_{i}}\right)^{r_{i}}=\prod_{k=0}^{r_{i}-1}\left(b_{\alpha_{i}}^{\dagger} b_{\alpha_{i}}-k\right)
$$

enters in the first line, and the identity

$$
\begin{gathered}
\left\langle\prod_{i=1}^{m}\left(b_{\alpha_{i}}^{\dagger}\right)^{r_{i}}\left(b_{\alpha_{i}}\right)^{r_{i}}\right\rangle_{\mathrm{G}}=\prod_{i=1}^{m}\left\langle\left(b_{\alpha_{i}}^{\dagger}\right)^{r_{i}}\left(b_{\alpha_{i}}\right)^{r_{i}}\right\rangle_{\mathrm{G}}=\prod_{i=1}^{m}\left\langle\prod_{k=0}^{r_{i}-1}\left(\mathcal{I}_{\alpha_{i}}-k\right)\right\rangle_{\mathrm{G}} \\
=\prod_{i=1}^{m}\left[r_{i} !\left(\left\langle\mathcal{I}_{\alpha_{i}}\right\rangle_{\mathrm{G}}\right)^{r_{i}}\right]=\prod_{i=1}^{m}\left[r_{i} !\left(\left\langle\mathcal{I}_{\alpha_{i}}\right\rangle_{0}\right)^{r_{i}}\right]
\end{gathered}
$$

in the second line. Furthermore the permutation-averaged matrix elements $\widetilde{A}_{\alpha_{1} \cdots \alpha_{m}}=$ $\sum_{P}(\mp 1)^{P} A_{\alpha_{P 1} \cdots \alpha_{P m}}^{\alpha_{1} \cdots \alpha_{m}}$ and $\widetilde{B}_{\alpha_{1} \cdots \alpha_{m}}=\sum_{P} B_{\alpha_{P 1} \cdots \alpha_{P m}, r_{P 1} \cdots r_{P m}}^{\alpha_{1} \cdots \alpha_{m}, r_{1} \cdots r_{m}}$ have been defined. 
From these results rather general sufficient conditions for the validity of the GGE predictions can be obtained, namely the factorization of initial-state expectation values of (a) products or (b) polynomials of the constants of motion $\mathcal{I}_{\alpha}$ :

$$
\begin{array}{lr}
\text { If }\left\langle\prod_{i=1}^{m} \mathcal{I}_{\alpha_{i}}\right\rangle_{0}=\prod_{i=1}^{m}\left\langle\mathcal{I}_{\alpha_{i}}\right\rangle_{0} & \text { then } \overline{\langle A\rangle}=\langle A\rangle_{\mathrm{G}} . \\
\text { If }\left\langle\prod_{i=1}^{m} \prod_{k=0}^{r_{i}-1}\left(\mathcal{I}_{\alpha_{i}}-k\right)\right\rangle_{0}=\prod_{i=1}^{m}\left[r_{i} !\left(\left\langle\mathcal{I}_{\alpha_{i}}\right\rangle_{0}\right)^{r_{i}}\right] & \text { then } \overline{\langle B\rangle}=\langle B\rangle_{\mathrm{G}} .
\end{array}
$$

These factorizations occur trivially for simple observables that involve no more than one factor of $\mathcal{I}_{\alpha}$. For example, this is the reason why the GGE (4.9) yields the correct longtime average for the double occupation of the $1 / r$ Hubbard model [126]. In summary, GGEs correctly describe the steady state for sufficiently uncorrelated initial states or sufficiently uncorrelated observables. This statement agrees in particular with the results of Ref. [152], where the GGE was shown to make the wrong predictions for correlations between bosonic occupation numbers.

As an application for the criteria (4.19) it is now shown that the GGE correctly describes expectation values of local observables for hard-core bosons on a chain of length $L$ (up to finite size corrections of order $1 / L$ ) for the same initial state as in Ref. [122]. In Ref. [122], hard-core bosons are studied on an lattice with alternating on-site potential $\Delta$,

$$
H_{h c b}=\sum_{\langle i, j\rangle} t_{i j} b_{i}^{\dagger} b_{i}+\Delta \sum_{i}(-1)^{i} b_{i}^{\dagger} b_{i},
$$

$\left(\left[b_{i}, b_{j}^{\dagger}\right]=\delta_{i j}, b_{i}^{2}=\left(b_{i}^{\dagger}\right)^{2}=0\right)$. The system in initially in the ground state, and $\Delta$ is suddenly switched off at $t=0$. Local observables $B$ are defined such that they contain only bosonic annihilation and creation operators $b_{i}$ and $b_{i}^{\dagger}$ that are restricted to sites $i$ in a finite segment $i \in\left[i_{\text {min }}, i_{\text {min }}+M\right]$. A typical example is the density-density correlation function $\left\langle b_{i}^{\dagger} b_{i} b_{j}^{\dagger} b_{j}\right\rangle$. To solve the hard-core bosons Hamiltonian, one uses the JordanWigner transformation,

$$
b_{i}^{\dagger}=c_{i}^{\dagger} \prod_{\beta=1}^{i-1} e^{-i \pi c_{\beta}^{\dagger} c_{\beta}}, \quad b_{i}=\prod_{\beta=1}^{i-1} e^{i \pi c_{\beta}^{\dagger} c_{\beta}} c_{i},
$$

which maps maps hard-core bosons to noninteracting fermions $c_{i}$ (with conserved momentum occupation numbers $c_{k}^{\dagger} c_{k}$ ). Bosonic observables thereby become a rather complicated many-fermion expressions, but from Eq. 4.21) it is apparent that a local observable in the above sense contains only fermionic operators $c_{i}$ for $i \in\left[i_{\min }, i_{\min }+M\right]$, and hence only finitely many terms. It is thus sufficient to consider a single term, $A=c_{i_{1}}^{\dagger} \ldots c_{i_{n}}^{\dagger} c_{j_{n}} \ldots c_{j_{n}}$, which can be cast in the form (4.11), where $\alpha_{i}$ and $\beta_{i}$ now denote fermionic momenta, and the coefficients $A_{\beta_{1} \cdots \beta_{m}}^{\alpha_{1} \cdots \alpha_{m}}$ are obtained from the expansion of local operators $c_{i}$ in terms of delocalized momentum states $c_{k}$, such that $\left|A_{k_{1}^{\prime} \cdots k_{m}^{\prime}}^{k_{1} \cdots k_{m}}\right| \leq$ const. $/ L^{m}$. The initial state is the ground state of a superlattice of periodicity two, and the superlattice is switched off 
at $t=0$. Hence the factorization (4.19a) occurs for all momenta apart from $k+\pi=k^{\prime}$, and the contribution of the remaining terms to the expectation value (4.14) is of order $1 / L$ due to the bound on the matrix elements. This proves the proposition, i.e., the GGE and the long-time average agree for all local observables for one-dimensional hard-core bosons after a quench of a superlattice potential. In Ref. [122 this agreement was found for some observables, but differences were observed for the bosonic one-particle density matrix $\rho_{i j}=\left\langle b_{i}^{\dagger} b_{j}\right\rangle$. The above argument thus generalizes these results and identifies the differences between the GGE and the time average of $\rho_{i j}$ as finite-size corrections.

\section{Degenerate energy levels}

In the previous subsection it was assumed that the degeneracy of energy levels is irrelevant [as defined above Eq. (4.13)], which allowed us to move from Eq. (4.4) to (4.5). Below an example is given for which this assumption does not hold (see Ref. [126]). In that case the expression (4.13) for the long-time average cannot be used, and thus neither (4.14a) nor 4.16a are available.

We consider a quench to $U=0$ in a general fermionic Hubbard model. Fermions (with spin $\sigma=\uparrow, \downarrow$ ) on a Bravais lattice (with $L$ lattice sites) are prepared in a correlated unpolarized initial state $\rho_{0}$ with fixed densities $n_{\uparrow}=n_{\downarrow}=n / 2$. The time evolution is governed by the free Hamiltonian

$$
H=\sum_{i j \sigma} V_{i j} c_{i \sigma}^{\dagger} c_{j \sigma}=\sum_{\boldsymbol{k} \sigma} \epsilon_{\boldsymbol{k}} c_{\boldsymbol{k} \sigma}^{\dagger} c_{\boldsymbol{k} \sigma}
$$

where $\boldsymbol{k}$ labels the crystal momentum. Periodic boundary conditions are assumed for simplicity. This Hamiltonian of the form 4.10, with the number operators $n_{\boldsymbol{k} \sigma}=c_{\boldsymbol{k} \sigma}^{\dagger} c_{\boldsymbol{k} \sigma}$ playing the role of the constants of motion $\mathcal{I}_{\alpha}$. In the following the steady-state expectation value of the double occupation $n_{i \uparrow} n_{i \downarrow}$ is considered.

Assuming again that the degeneracy of energy levels is irrelevant, the long-time average 4.13 is obtained, using the basis $|\boldsymbol{m}\rangle=\prod_{k \sigma}\left(c_{\boldsymbol{k} \sigma}^{\dagger}\right)^{m_{\boldsymbol{k} \sigma}}|0\rangle$,

$$
\overline{\left\langle n_{i \uparrow} n_{i \downarrow}\right\rangle}=\sum_{\boldsymbol{m}}\left\langle\boldsymbol{m}\left|\rho_{0}\right| \boldsymbol{m}\right\rangle \frac{1}{L^{2}} \sum_{\boldsymbol{k}^{\prime}} m_{\boldsymbol{k} \uparrow} m_{\boldsymbol{k}^{\prime} \downarrow}=\operatorname{Tr}\left[\frac{\rho_{0}}{L^{2}} \sum_{i j} n_{i \uparrow} n_{j \downarrow}\right]=n_{\uparrow} n_{\downarrow}=\frac{n^{2}}{4} .
$$

The same value is obtained from the canonical and grand-canonical ensemble, and also from the generalized Gibbs ensemble which uses the number operators $n_{\boldsymbol{k} \sigma}$ as constants of motion:

$$
\left\langle n_{i \uparrow} n_{i \downarrow}\right\rangle_{\mathrm{G}}=\frac{1}{L^{2}} \sum_{\boldsymbol{k} \boldsymbol{k}^{\prime}}\left\langle n_{k \uparrow}\right\rangle_{\mathrm{G}}\left\langle n_{k^{\prime} \downarrow}\right\rangle_{\mathrm{G}}=\frac{1}{L^{2}} \sum_{\boldsymbol{k} \boldsymbol{k}^{\prime}}\left\langle n_{k \uparrow}\right\rangle_{0}\left\langle n_{k^{\prime} \downarrow}\right\rangle_{0}=n_{\uparrow} n_{\downarrow}=\frac{n^{2}}{4} .
$$

Thus one can conclude that the double occupation thermalizes to the value $n^{2} / 4$ after a quench to $U=0$ in any Hubbard model, provided that the degeneracy of energy levels is indeed irrelevant. 
This statement disagrees with exact results for the $1 / r$ Hubbard chain which have been derived in Ref. [126] When the $1 / r$ Hubbard chain is quenched from $U=\infty$ to $U=0$, the long-time limit of the double occupancy is given by $d_{\infty}=n^{2}(3-2 n) / 6$. This differs from the long-time average (4.23). The reson for this behavior is that the linear dispersion $\epsilon_{\boldsymbol{k}}=t k$ of the $1 / r$ hopping leads to a massive degeneracy for the free-fermion energy eigenstates $|\boldsymbol{m}\rangle$, such that the long-time limit is not given by Eq. 4.13).

\subsection{Prethermalization}

We now proceed to the question how the relaxation to thermal equilibrium proceeds in time. In this context one frequently encounters the prethermalization phenomenon [153], both in classical and quantum systems. Prethermalization implies that a system relaxes to a quasistationary but still nonthermal state on a rather short timescale, while the subsequent thermalization takes place on a much longer timescale. In the quasistationary intermediate state several quantities such as the kinetic and potential energy can have reached almost their thermal expectation values, but others, such as the momentum distribution function, are far from equilibrium.

A rather general argument for the existence of prethermalization can be given when the Hamiltonian is close to integrability, where thermalization is inhibited by infinitely many constants of motion. In this case one can perform a perturbation expansion of the Hamiltonian in the vicinity of the integrable point. High-order terms in the expansion become effective only on very long timescales, while the dynamics that is governed by the leading terms is constrained by many conserved quantities. For example, for the classical field-theory of Ref. [154] the time evolution on intermediate timescales is governed by a Hartree mean-field theory, which allows nonthermal steady states due to infinitely many conserved correlation functions. Moeckel et al. studied the prethermalization after quenches in the Hubbard model to weak interaction $U \ll V$ by a similar argument [127, [128]. They use the flow equation technique and keep terms up to order $(U / V)^{2}$, such that the Hamiltonian is transformed to noninteracting quasiparticles. In this approximation, the system can thus not thermalize, but it relaxes to a nonthermal stationary state on the timescale $V / U^{2}$. This is seen explicitly in the momentum distribution, which retains a jump at the Fermi edge while it would be a smooth function in equilibrium at finite temperature. The jump turns out to be exactly twice as large as in the equilibrium ground state for interaction $U$. It is then argued that the neglected terms in the expansion lead to thermalization on longer timescales $V^{3} / U^{4}$. An interesting question, which is not persued here, is whether the nonthermal quasistationary state can be described by a generalized Gibbs ensemble of the transformed Hamiltonian.

In the following we will show that a similar behavior as for the weak-coupling limit occurs in the Hubbard model (1.1) for $V \ll U$, i.e., close to the atomic limit $V=0$, where relaxation to the thermal state is trivially impossible. The system then remains trapped in a nonthermal quasistationary state on the long timescale $U / V^{2}$ for a general initial state $\left|\psi_{0}\right\rangle$. Similar results have been obtained for the Bose-Hubbard model [124]. To prove this, we use the standard unitary transformation $\bar{A}=e^{-S} A e^{S}$ [155] for which 
the double occupation $\bar{D}=\sum_{i} \bar{n}_{i \uparrow} \bar{n}_{i \downarrow}$ of the dressed fermions $\bar{c}_{i \sigma}$ is conserved, $[H, \bar{D}]$ $=0$. This transformation was originally constructed to obtain an effective low-energy Hamiltonian for the Hubbard model in the strong-coupling limit (the $t$ - $J$ model). The antihermitian matrix is expressed in terms of a decomposition the hopping term $K=$ $\sum_{i j \sigma} t_{i j \sigma} c_{i \sigma}^{\dagger} c_{j \sigma}=H_{k i n} / V$ (i.e., $V_{i j \sigma}=V t_{i j \sigma}$ ) into operators $K_{p}$ that change the double occupation by $p$, i.e.,

$$
\begin{aligned}
& K_{+}=\sum_{i j \sigma} t_{i j \sigma} c_{i \sigma}^{\dagger} c_{j \sigma}\left(1-n_{j \bar{\sigma}}\right) n_{i \bar{\sigma}} \\
& K_{-}=\sum_{i j \sigma} t_{i j \sigma} c_{i \sigma}^{\dagger} c_{j \sigma} n_{j \bar{\sigma}}\left(1-n_{i \bar{\sigma}}\right)=\left(K_{+}\right)^{\dagger} \\
& K_{0}=\sum_{i j \sigma} t_{i j \sigma} c_{i \sigma}^{\dagger} c_{j \sigma}\left(1-n_{i \bar{\sigma}}-n_{j \bar{\sigma}}+2 n_{j \bar{\sigma}} n_{i \bar{\sigma}}\right)=K-K_{-}-K_{+} .
\end{aligned}
$$

To leading order on then has [155]

$$
S=(V / U)\left(\bar{K}_{+}-\bar{K}_{-}\right)+(V / U)^{2}\left[\bar{K}_{+}+\bar{K}_{-}, \bar{K}_{0}\right]+\mathcal{O}\left(V^{3} / U^{3}\right) .
$$

Double occupation and Hamiltonian can now be expressed in the transformed (dressed) fermions $\bar{c}$ using the Baker-Hausdorff formula

$$
A=e^{S} \bar{A} e^{-S}=\bar{A}+[S, \bar{A}]+\frac{1}{2 !}[S,[S, \bar{A}]]+\ldots
$$

and the commutation relation

$$
\left[D, K_{ \pm}\right]= \pm K_{ \pm}
$$

which follows from the definition 4.25 . We obtain

$$
\begin{aligned}
& D=\bar{D}+(V / U)\left(\bar{K}_{+}+\bar{K}_{-}\right)+\mathcal{O}\left(V^{2} / U^{2}\right) \\
& H=U \bar{D}+V \bar{K}_{0}+\mathcal{O}\left(V^{2} / U\right) .
\end{aligned}
$$

This is inserted into the expression $d(t)=\left\langle e^{i H t} D e^{-i H t}\right\rangle_{0} / L$ to obtain the time evolution of the double occupancy,

$$
L d(t)=\langle\bar{D}\rangle_{0}+\frac{V}{U}\left\langle e^{i t\left(U \bar{D}+V \bar{K}_{0}\right)}\left(\bar{K}_{+}+\bar{K}_{-}\right) e^{-i t\left(U \bar{D}+V \bar{K}_{0}\right)}\right\rangle_{0}+\mathcal{O}\left(\frac{V^{2}}{U^{2}}, \frac{t V^{3}}{U^{2}}\right)
$$

Here $\langle\cdot\rangle_{0}=\left\langle\psi_{0}|\cdot| \psi_{0}\right\rangle$ denote the initial state expectation value. The error $\mathcal{O}\left(t V^{3} / U^{2}\right)$, which is due to omitted terms in the exponentials $e^{ \pm i H t}$, is irrelevant in comparison to the leading terms if $t \ll U / V^{2}$. One can now factorize the exponential $e^{i t\left(U \bar{D}+V \bar{K}_{0}\right)}$ because $\left[D, K_{0}\right]=0$, and calculate the time-dependent operators $e^{i t U \bar{D}} \bar{K}_{ \pm} e^{-i t U \bar{D}}$ from Eq. 4.28. The final result is

$$
d(t)=d_{\text {stat }}-\frac{2 V}{U} \operatorname{Re}\left[e^{i t U} R(t V)\right]+\mathcal{O}\left(\frac{V^{2}}{U^{2}}, \frac{t V^{3}}{U^{2}}\right),
$$


where

$$
R(t V)=\left\langle e^{i t V K_{0}} K_{+} e^{-i t V K_{0}}\right\rangle_{0} / L
$$

and

$$
\begin{aligned}
d_{\text {stat }} & =d(0)-\Delta d, \\
\Delta d & =-\frac{1}{L} \sum_{i j \sigma} t_{i j \sigma}\left\langle c_{i \sigma}^{\dagger} c_{j \sigma}\left(n_{i \bar{\sigma}}-n_{j \bar{\sigma}}\right)^{2}\right\rangle_{0} .
\end{aligned}
$$

In this expression dressed fermions $\bar{c}$ were again replaced by original fermions $c$ with an error of the same order as already in Eq. 4.32.

The envelope function $R(t V)$ of the oscillating term decays to zero for $t \gg 1 / V$. To see this, insert an eigenbasis $K_{0}|m\rangle=k_{m}|m\rangle, R(t V)=\sum_{m, n}\langle\mid n\rangle\langle m \mid\rangle_{0} e^{i t V\left(k_{m}-k_{n}\right)}$ $\left\langle n\left|K_{+}\right| m\right\rangle$. In this expression all oscillating terms dephase in the long-time average, so that only energy-diagonal terms contribute to the sum. Furthermore, it follows from $\left[K_{0}, D\right]=0$ that $D$ is a good quantum number of $|n\rangle$ such that $\left\langle n\left|K_{+}\right| n\right\rangle=0$. Thus we can show that $R(t V)$ vanishes in the long-time limit if we assume that this limit exitst (it is then equal to the long-time average) and if accidental degeneracies between sectors of different $D$ are irrelevant.

Eqs. 4.32)-(4.34) thus shows that $d(t)$ relaxes through damped oscillations to the quasistationary value (4.34) on a timescale $1 / V$, while corrections occur only on the much longer timescale $U / V^{2}$. This result is valid for arbitrary initial states. To show that Eq. (4.34) is indeed different from the thermal value, we have to compare it to the double occupancy in a thermal ensemble with the same internal energy $\left\langle\psi_{0}|H| \psi_{0}\right\rangle$ [cf. Eq. 4.8].

For the remainder of this section we assume that the initial state $\left|\psi_{0}\right\rangle$ is a noninteracting ground state, which is a situation that is studied numerically in Ch. 5 and 6 . In this case, the expectation value in Eq. 4.34b factorizes with respect to spin,

$$
\Delta d=-\frac{1}{L} \sum_{i j \sigma} t_{i j \sigma}\left\langle c_{i \sigma}^{\dagger} c_{j \sigma}\right\rangle_{0}\left\langle\left(n_{i \bar{\sigma}}-n_{j \bar{\sigma}}\right)^{2}\right\rangle_{0} .
$$

A further simplification occurs in the limit of infinite dimensions, where we have $\left\langle n_{i \bar{\sigma}} n_{j \bar{\sigma}}\right\rangle_{0}$ $=\left\langle n_{i \bar{\sigma}}\right\rangle_{0}\left\langle n_{j \bar{\sigma}}\right\rangle_{0}$ for $i \neq j$. For a homogeneous phase with $\left\langle n_{i \bar{\sigma}}\right\rangle_{0} \equiv n_{\bar{\sigma}}$, this gives

$$
\Delta d=-\frac{1}{L} \sum_{\sigma} 2 n_{\bar{\sigma}}\left(1-n_{\bar{\sigma}}\right) \frac{E_{k i n, 0}^{\sigma}}{V},
$$

which is proportional to the kinetic energy in a noninteracting state [Eq. (3.33)]. The thermal value can be obtained from a high-temperature expansion, because the excitation energy with respect to the new ground state is $\mathcal{O}(U)$ when $\left|\psi_{0}\right\rangle$ is a noninteracting initial state, such that the effective temperature [cf. Eq. 4.8] ] is large, $T_{*} \gg V$. One obtains 74

$$
\begin{aligned}
d_{\mathrm{th}} & =d(0)-\Delta d_{t h} \\
\Delta d_{t h} & =-\sum_{\sigma} \frac{E_{k i n, 0}^{\sigma}}{V L}
\end{aligned}
$$


In conclusion, the above argument shows that $d(t)$ relaxes to a nonthermal value on the fast timescale $1 / V$, although thermalization is expected for the nonintegrable Hubbard model. The constrained dynamics on the timescale $U / V^{2}$ should be visible in other observables.

On the other hand, the timescale for thermalization cannot be determined from the above argument. In particular, we do not claim that the neglected terms of the expansion lead to thermalization on the timescale $U / V^{2}$. Quite opposite: the unitary transformation can in principle be constructed to arbitrary high order [156], such that the conservation of $\bar{D}$ is violated only by nonperturbative effects in this expansion and thermalization of $d$ may occur only on exponentially long timescales $\exp ($ const. $U / V)$ [157].7] However, the long-time dynamics remains inaccessible at the moment and it is thus unclear how, and whether, the strong-coupling expansion breaks down. It is therefore interesting to note that for the Falicov-Kimball model (Sec. 5.3.4) and the $1 / r$ Hubbard model [126] the exact long-time limit of $d(t)$, which is nonthermal, coincides with Eqs. (4.34a) and (4.35) to lowest order in $V / U$. There are no nonperturbative corrections in this case which lead to thermalization.

\subsection{Relaxation dynamics of nearly integrable systems}

In the previous section it was demonstrated that the dynamics of a nonintegrable system can resemble the nonergodic behavior of integrable systems on very long timescales, provided that the former is obtained by a small perturbation of an integrable system. While thermalization was assumed for long times, a proof could not be given. This raises the question whether there is some room for truly nonergodic behavior of a nonintegrable system on infinitely long timescales, even in the thermodynamic limit. We now discuss some of the concepts related to this question.

A similar unitary perturbation expansion as in the previous section can in principle be formulated quite generally for systems that are close to an integrable point. For this purpose consider again the effective noninteracting Hamiltonian (4.10) with conserved single-particle occupations $\mathcal{I}_{\alpha}=a_{\alpha}^{\dagger} a_{\alpha}$ (bosons of fermions), but this time allow for a perturbation term $W$,

$$
H=H_{\mathrm{eff}}+\gamma W, \quad\left[H_{\mathrm{eff}}, \mathcal{I}_{\alpha}\right]=0, \quad\left[W, \mathcal{I}_{\alpha}\right] \neq 0 .
$$

For small perturbations $\gamma \ll 1$ (the unit of energy is set by the largest scale in $H_{\text {eff }}$ ) one can construct a unitary mapping $\bar{A}=e^{S} A e^{-S}$ ), such that the modified constants of motion $\overline{\mathcal{I}}_{\alpha}$ are still conserved, i.e.,

$$
\left[\overline{\mathcal{I}}_{\alpha}, H\right]=0 \Leftrightarrow\left[\mathcal{I}_{\alpha}, e^{S} H e^{-S}\right]=0
$$

The generator $S$ is constructed order by order as a power series in $\gamma$. For this the series

\footnotetext{
${ }^{7}$ When the higher order terms of $S$ are included in the above argument, the error $\propto \frac{t V^{3}}{U^{2}}$ in Eq. 4.31 , is absent.
} 
representation

$$
S=\sum_{n=1}^{\infty} \frac{\gamma^{n}}{n !} S_{n},
$$

and the Baker-Hausdorff formula $e^{S} H e^{-S}=H+[S, H]+\frac{1}{2}[S,[S, H]]+\ldots$, are used to derive a power series for $e^{S} H e^{-S}$,

$$
e^{S} H e^{-S}=H_{\mathrm{eff}}+\gamma\left(\left[S_{1}, H_{\mathrm{eff}}\right]+W\right)+\frac{\gamma^{2}}{2}\left(\left[S_{2}, H_{\mathrm{eff}}\right]+\left[S_{1}, 2 W+\left[S_{1}, H_{\mathrm{eff}}\right]\right]\right)+\ldots
$$

Because the $\mathcal{I}_{\alpha}$ and $H_{\text {eff }}$ are pairwise commuting, one can assume a common eigenbasis $|m\rangle$

$$
H_{\text {eff }}|m\rangle=E_{m}^{0}|m\rangle, \quad \mathcal{I}_{\alpha}|m\rangle=m_{\alpha}|m\rangle .
$$

In order for each $\mathcal{I}_{\alpha}$ to commute with $e^{S} H e^{-S}$ it is thus sufficient that the coefficient of each power $\gamma^{n}$ in 4.41) is diagonal in this basis. This can easily be satisfied by solving a linear equation for each term. The first two terms are given by

$$
\begin{aligned}
\left(S_{1}\right)_{n m} & =\frac{1-\delta_{n m}}{E_{n}^{0}-E_{m}^{0}} \widetilde{W}_{n m} \\
\left(S_{2}\right)_{n m} & =\frac{1}{2} \frac{1-\delta_{n m}}{E_{n}^{0}-E_{m}^{0}}\left(\left[S_{1}, \widetilde{W}\right]\right)_{n m} \\
\ldots &
\end{aligned}
$$

where $\widetilde{W}_{n m}=W_{n m}\left(1-\delta_{n m}\right)$, i.e., the off-diagonal part of the disturbing operator. To calculate the time evolution of an observable $O(t)$ for times $t \ll 1 / \gamma^{N}$, one can use the unitary transformation up to order $N$, i.e., $S=S_{N} \equiv \sum_{n=1}^{N} \gamma^{n} S_{n} / n$ !,

$$
\begin{aligned}
O(t) & \equiv\left\langle\psi_{0}\left|e^{i H t} O e^{-i H t}\right| \psi_{0}\right\rangle=\left\langle\psi_{0}\left|e^{S} e^{i \bar{H} t} \bar{O} e^{-i \bar{H} t} e^{-S}\right| \psi_{0}\right\rangle \\
& =\left\langle\psi_{0}\left|e^{S} e^{i\left(H_{\text {eff }}+h_{\text {diag }}\right) t} \bar{O} e^{-i\left(H_{\text {eff }}+h_{\text {diag }}\right) t} e^{-S}\right| \psi_{0}\right\rangle+\mathcal{O}\left(t \gamma^{n+1}\right) .
\end{aligned}
$$

In the second line it was used that $S_{N}$ is constructed such that $\bar{H}=H_{\text {eff }}+h_{\text {diag }}+\mathcal{O}\left(\gamma^{N+1}\right)$ with an energy-diagonal term $h_{\text {diag }}$.

How close is Eq. (4.44) to the thermal value of $O$ for times $t \ll 1 / \gamma^{N}$ ? This depends on the initial state $\left|\psi_{0}\right\rangle$ and the observable, but the two values do not coincide in general. For example, let $O$ be the momentum occupation $n_{\alpha}=c_{\alpha}^{\dagger} c_{\alpha}$, such that $n_{\alpha}(t)=\left\langle n_{\alpha}\right\rangle_{0}+$ $\mathcal{O}(\gamma)$, where the leading order corrections come from an expansion of $O$ and $e^{S}$ in (4.44). However, if the excitation energy of $\left|\psi_{0}\right\rangle$ is $\mathcal{O}(1)$, also the momentum occupation will differ from the initial one by $\mathcal{O}(1)$ and not by $\mathcal{O}(\gamma)$. Hence the thermal value is not approached for times $t \ll 1 / \gamma^{N}$ for any $N$. In other words, thermalization is a nonperturbative effect that cannot be described within perturbation theory around the integrable point.

It remains open whether there is a truly nonthermal stationary state below some threshold of $\gamma$, or whether thermalization occurs on exponentially long timescales $\propto$ $\exp ($ const. $/ \gamma)$. The answer to this question is known in general for quantum systems. 
However, the classical analog of the perturbation around an integrable point was already considered by Poincaré in the context of celestial mechanics at the end of the 19th century [113. He proved that the existence of small energy denominators [cf. Eq. (4.43)] leads to a breakdown of the classical perturbation theory around the integrable point. There are no integrals of motion besides the energy that are analytic functions of the perturbation $\gamma$. However, this does not yet imply chaotic behavior for any $\gamma>0$. The problem of dynamics close to an integrable point of a classical was finally resolved by a fundamental theorem by Kolmogorov, Arnold, and Moser (KAM) $8^{8}$

The KAM theorem states that when an integrable Hamiltonian system is slightly perturbed, there remains a set of invariant tori, which covers most of phase space (its volume is actually controlled $\gamma$ ). Nevertheless this set is nowhere dense in phase space (a situation that clearly cannot be describe by perturbation theory in $\gamma$ ) and in the remaining region of phase space the motion is ergodic. The KAM estimate for the breakdown of integrability can hardly be evaluated analytically, and one has to resort to numerical calculations. Numerous computer studies of the chaos-nonchaos transition for classical systems exist in the literature, starting from the seminal paper by Henon and Heiles [158]. Also for the FPU problem there are numerical indications that chaotic behavior occurs below some threshold of the excitation energy [117]. Interestingly, this threshold seems to vanish with increasing system size, which would imply that in the thermodynamic limit ergodic behavior occurs for arbitrarily small excitation energy [117]. In a different context, a transition from ergodic to nonergodic behavior is obtained for classical viscous liquids within the mode-coupling theory [159], where it is proposed as an ideal form of the glass transition. In quantum systems, a threshold for chaos and thermalization, which is finite in the thermodynamic limit, is observed within a mean-field treatment of the one-dimensional Bose Hubbard model [160].

A completely different approach to the transition from ergodic to nonergodic behavior is to study the breakdown of the eigenstate thermalization hypothesis (4.7) close to an integrable point of the Hamiltonian [130]. This idea is illustrated in the following by an exact diagonalization of a finite two-dimensional cluster of the Hubbard model $(L=$ 8 sites, 4 particles per spin direction, Hilbert space dimension $D=4900$ ). In order to check the validity of the (4.7), the expectation value of the momentum occupation $n_{\boldsymbol{k}}=$ $\sum_{\sigma i} e^{i \boldsymbol{k}\left(\boldsymbol{R}_{i}-\boldsymbol{R}_{j}\right)} c_{i \sigma}^{\dagger} c_{j \sigma}$ is evaluated at an incommensurate momentum $\boldsymbol{k}_{1}=(0.657,0.234)$ in each eigenstate $|n\rangle$. This requires full diagonalization of the cluster, and not only determination of the ground state, and hence we are restricted to relatively small clusters. The expectation values $\left(n_{\boldsymbol{k}}\right)_{m}=\left\langle m\left|n_{\boldsymbol{k}}\right| m\right\rangle$ are plotted in Fig. 4.2 against the energy eigenvalue $E_{m}$. If the (4.7) is fulfilled, one would expect that the $\left\langle m\left|n_{\boldsymbol{k}}\right| m\right\rangle$ only depend on the energy $E_{m}$ and thus fall on a straight line in the diagram, as in Ref. [129]. This is apparently not true for the integrable case $U=0$, but improves with increasing $U$. To quantitatively judge the validity as a function of $U$, the distribution $P_{A}(a, E)$ of the

\footnotetext{
${ }^{8}$ The theorem was suggested by Kolmogorov in 1954 and proven by Arnold (1963) and Moser (1963). For an introduction, consider Ref. [108.
} 

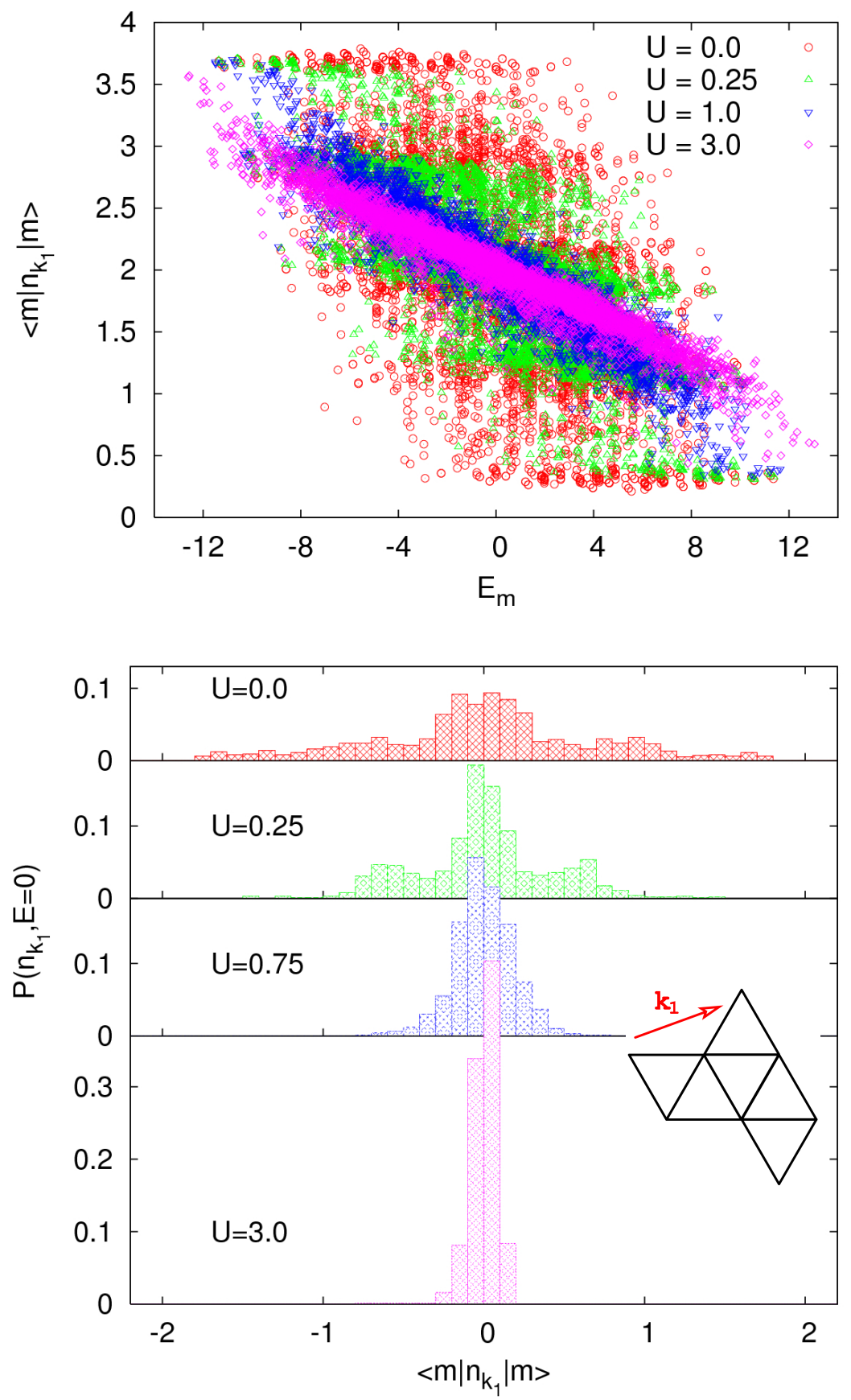

Figure 4.2: Top: Expectation value $\left\langle m\left|n_{\boldsymbol{k}_{1}}\right| m\right\rangle$ of of the momentum occupation $n_{\boldsymbol{k}_{1}}=$ $\sum_{\sigma i} e^{i \boldsymbol{k}\left(\boldsymbol{R}_{i}-\boldsymbol{R}_{j}\right)} c_{i \sigma}^{\dagger} c_{j \sigma}$ at $\boldsymbol{k}_{1}=(0.657,0.234)$ in the energy eigenstates $|m\rangle$ of the Hubbard model on a finite cluster (inset in lower panel), as a function of interaction $U$. Bottom: Distribution function (4.45) for $\left\langle m\left|n_{\boldsymbol{k}_{1}}\right| m\right\rangle$ and $E=0$, integrated over bins of width $\delta n$ $=0.1$. Since the system is finite, we take $\Delta E=1$, and approximate $\left\langle n_{\boldsymbol{k}_{1}}\right\rangle_{\text {mic }}$ by a linear least square fit of the data points in the upper panel in the energy range $-\Delta E \leq E \leq$ $\Delta E$. 
expectation values $A_{m}=\langle m|A| m\rangle$ around the microcanonical average $\langle A\rangle_{\text {mic }}(E)$,

$$
\begin{aligned}
P_{A}(a, E) & =\frac{\sum_{m:\left|E-E_{m}\right| \leq \Delta E} \delta\left(A_{m}-\langle A\rangle_{m i c}(E)-a\right)}{\sum_{m:\left|E-E_{m}\right| \leq \Delta E} 1} \\
\langle A\rangle_{m i c}(E) & =\frac{\sum_{m:\left|E-E_{m}\right| \leq \Delta E} A_{m}}{\sum_{m:\left|E-E_{m}\right| \leq \Delta E} 1}
\end{aligned}
$$

In Fig. 4.2 this distribution is plotted for the observable $A=n_{\boldsymbol{k}}$. There is a clear tendency for a narrowing of the distribution $P_{A}(E, a)$ when one departs from the noninteracting state.

The reslts present demonstrate the breakdown of the eigenstate thermalization close to an integrable point of the Hamiltonian. This breakdown is observed in the form of a crossover from a broad distribution of expectation valued $\langle m|A| m\rangle$ to a narrow one. Similar results were recently reported for hard-core bosons [130]. Using large Hilbert space sizes up to 30000 it was shown that the crossover strongly depends on the energy of the system. This prooves that the validity of (4.7) can be taken as a measure for a breakdown of ergodicity close to an integrable point. Surely, the ultimate goal would be to investigate the scaling of $P_{A}(a, E)$ with system size and thus estimate whether some transition in $P_{A}(a, E)$ can be observed at a finite threshold energy and coupling, indicating either a true change from nonergodic to ergodic behavior, or a profound change in the relaxation behavior. This is still out of reach within the currenlty accessible Hilbert space size. 


\section{Chapter 5}

\section{Interaction quench in the Falicov-Kimball model}

\subsection{The Falicov-Kimball model}

The Falicov-Kimball model was introduced by Falicov and Kimball in 1969 [161 as a simple many-body model which exhibits a metal-insulator transition, and studied in a series of subsequent papers [162, 163]. It describes itinerant and immobile electrons on a lattice that interact via a local Coulomb repulsion $U$. The model can also be obtained as a limiting case of the Hubbard model in which the hopping for one spin species is set to zero; as such it had already been considered before 1969 by Hubbard [1] in order to obtain an approximate solution of the Hubbard model. The general considerations that have been made in previous chapters for Hubbard-type models with spin-dependent hopping remain valid for the Falicov-Kimball model, in particular the the diagrammatic rules for perturbation theory (Sec. 2.4) and the strong-coupling expansion around the atomic limit, where both spin species are immobile (Sec. 4.4). Throughout this chapter, the Hamiltonian is considered in the notation

$$
H=\sum_{i j} V_{i j} c_{i}^{\dagger} c_{j}+U \sum_{i} n_{i}^{f} n_{i}^{c}-\mu \sum_{i} n_{i}^{c}-\left(\mu-E_{f}\right) \sum_{i} n_{i}^{f}
$$

where $c_{i}^{(\dagger)}$ and $f_{i}^{(\dagger)}$ are annihilation (creation) operators for the itinerant and immobile electrons, respectively, and $n_{i}^{c}=c_{i}^{\dagger} c_{i}\left(n_{i}^{f}=f_{i}^{\dagger} f_{i}\right)$ are their local densities. Hopping between sites $i$ and $j$ (with amplitude $V_{i j}=V t_{i j}$ ) is possible only for the mobile particles. The hopping strength $V=1$ is chosen as energy unit.

The Falicov-Kimball model can be considered in two modes which imply rather different physical interpretations. Either (i) the total number of electrons $n_{f}+n_{c}$ is fixed, but the individual occupation of mobile and immobile electrons changes as a function of temperature and interaction $U$ (then $E_{f}$ is fixed, and $\mu$ determines the total particle number). This change in the nature of the carriers leads to the metal-insulator transition that has been discussed by the inventors of the model [161, 162]. (ii) On the other hand, the number of $c$ and $f$ electrons can be fixed separately by choosing $\mu$ and $E_{f}$ as 
independent variables. In this case the model exhibits a transition to long-range charge order at certain fillings and low temperatures [164, 165, 166]. This transition has been interpreted as a simple model for crystallization, where the immobile particles represent the heavy atoms, and the mobile particles the lighter electrons [166]. Some exact results are summarized in the review by Gruber and Macris [167.

The Falicov-Kimball model could be realized with cold atomic gases. The depth of the optical lattice depends on the detuning of the laser frequency with respect to the absorption frequencies of the atoms, and it is thus easy to mix two species of atoms with different hopping matrix elements. The introduction of localized particles into an optical lattice has already been used as an experimental realization of disorder in ultracold atomic gases [168]. Compared to other approaches like using laser speckle pattern this has the advantage of a rather short correlation length of the disorder. However, the preparation of an annealed disorder state of the Falicov-Kimball model (see below) as compared to quenched disorder, which is often assumed for localized atoms on an optical lattice [169] might be more difficult. One could try to achieve this by slowly switching off one of the hopping matrix elements.

However, apart from few examples such as valence change materials [43] the FalicovKimball model is a often rather crude approximation for the description of real materials. Nevertheless the model has played an important role in condensed matter physics as a benchmark for DMFT. Soon after Metzner and Vollhardt [49] introduced the limit of infinite dimensions to correlated fermion systems, Brandt and Mielsch [47] proved that the Falicov-Kimball model can be mapped to a local problem in a self-consistent field in this limit, and thus provided the solution of this model in $d=\infty$. This was probably the first application of DMFT, two years before a similar mapping was done for the Hubbard model [48]. Another approach of deriving an exact mean-field Hamiltonian of the Falicov-Kimball model was found by van Dongen and Vollhardt [170]. Since then various dynamic and static equilibrium properties of the Falicov-Kimball have been calculated with DMFT [171]. Most important for this thesis is the case of half-filling for both $c$ and $f$ particles, $n_{c}=n_{f}=\frac{1}{2}$. For this case, DMFT gives a charge-ordered state on a bipartite lattice at low temperatures [47, 172, 173]. In the homogeneous phase, a Mott-like metalinsulator transition occurs, where a gap is opened in the single-particle spectrum when $U$ is increased above a critical value $U=U_{c}$ [174, 175] (Fig. 5.1). Remarkably, the spectrum of the homogeneous phase of the Falicov-Kimball model is independent of temperature.

What makes the Falicov-Kimball model a correlated model and thus ultimately causes these phase transitions is the fact that, although the $f$ particles are immobile, the equilibrium state is not simply a random configuration of $f$-particles, but rather a state with annealed disorder. The $f$-particle configuration minimizes the energy of $c$-particles. More precisely, the thermal state $e^{-\beta H} / Z$ can be written as an disorder average over all possible configurations $\left\{n_{i}^{f}\right\}$ of localized particles, where the weight of each configuration is determined by the free energy of the $c$-particles,

$$
\frac{e^{-\beta H}}{Z}=\sum_{\left\{n_{i}^{f}\right\}} W\left[\left\{n_{i}^{f}\right\}\right] P_{\left\{n_{i}^{f}\right\}} \frac{e^{-\beta H_{c}\left[\left\{n_{i}^{f}\right\}\right]}}{Z_{c}\left[\left\{n_{i}^{f}\right\}\right]} .
$$



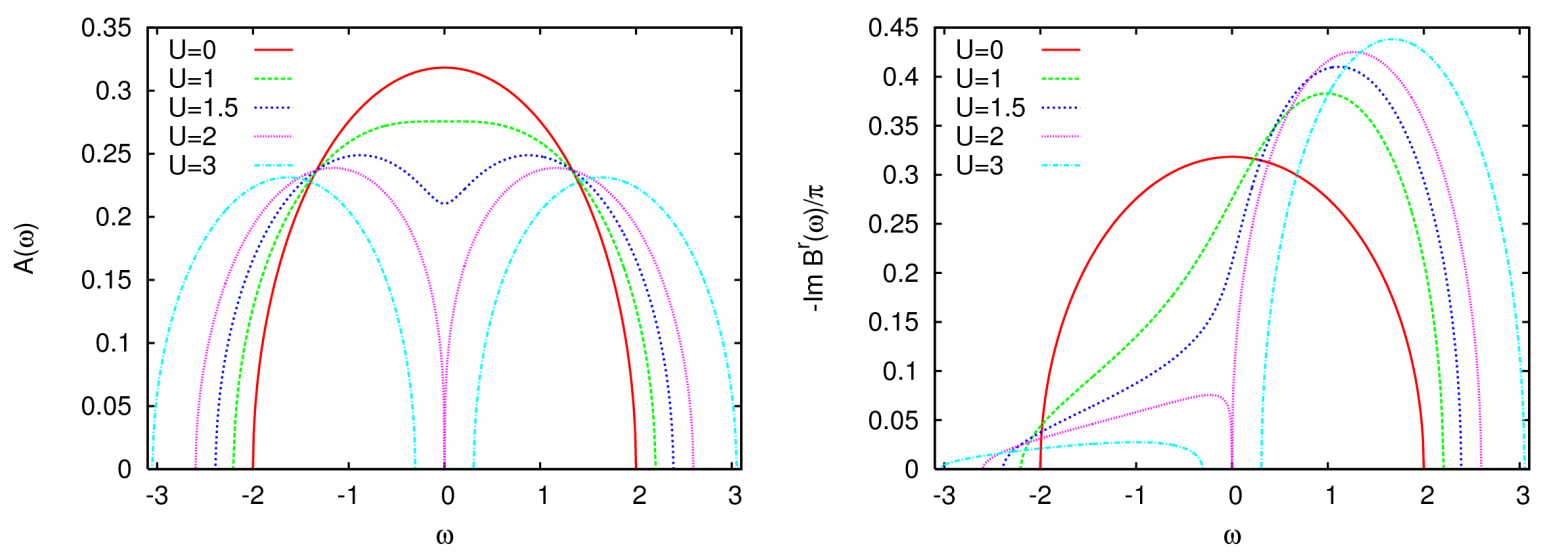

Figure 5.1: Spectrum of the local Green function $g^{\mathrm{r}}(\omega)(\mathrm{a})$, and of the local Green function $r^{\mathrm{r}}(\omega)$ at sites that are occupied with one $f$-particle (b). [DMFT for the homogeneous equilibrium phase at half-filling $\left(n_{c}=n_{f}=\frac{1}{2}\right)$, using a semielliptic density of states (3.18) with quarter bandwidth $V=1$, cf. Ref. [174], or Eqs. 5.25) below].

Here $H_{c}\left[\left\{n_{i}^{f}\right\}\right]$ is the (quadratic) Hamiltonian of the $c$-particles for given $\left\{n_{i}^{f}\right\}, Z_{c}\left[\left\{n_{i}^{f}\right\}\right]=$ $\operatorname{Tr}\left[e^{-\beta H_{c}\left[\left\{n_{i}^{f}\right\}\right]}\right]$,

$$
P_{\left\{n_{i}^{f}\right\}}=\prod_{i}\left(f_{i}^{\dagger} f_{i}\right)^{n_{i}^{f}}\left(1-f_{i}^{\dagger} f_{i}\right)^{1-n_{i}^{f}}
$$

is the projector on the configuration $\left\{n_{i}^{f}\right\}$, and

$$
W\left[\left\{n_{i}^{f}\right\}\right]=\frac{Z_{c}\left[\left\{n_{i}^{f}\right\}\right]}{Z}
$$

is its weight. This can be checked directly by comparing matrix elements on both sides of Eq. (5.2) in a product basis.

This annealed disorder leads to charge-ordered phases in the Falicov-Kimball model. However, for the homogeneous phase the DMFT equations turn out to be the same as the coherent-potential approximation (CPA). The CPA can be used as an approximate description for models with quenched binary disorder, where all weights $W\left[\left\{n_{i}^{f}\right\}\right]$ are equal (for a review of the CPA, cf. [176]). The CPA becomes exact in the limit of infinite dimensions [60]. Although the equivalence of the CPA solution and the homogeneous phase of the Falicov-Kimball model is not assumed from the beginning, but results from the DMFT solution, it implies all results that will be derived below for the homogeneous phase of the Falicov-Kimball model hold also for a quenched disorder system which is treated with CPA. This should be kept in mind for the interpretation of our results.

The Falicov-Kimball model was reconsidered whenever new developments were made within DMFT, e.g., for inhomogeneous DMFT [177], bosonic DMFT [55], and, most important for this work, nonequilibrium DMFT [43, 71, 68]. In all cases DMFT simplifies for the Falicov-Kimball model because the effective single-site problem is exactly solvable. In this chapter an interaction quench in the Falicov-Kimball model is considered, for which 
an analytical DMFT solution can be achieved [71, 73]. In particular, this allows one to study the long-time limit.

We assume that the system is prepared in thermal equilibrium at temperature $T$ for times $t<0$; at $t=0$ the interaction is suddenly switched from the value $U_{-}$to a new value $U_{+}$, so that the time evolution for $t \geq 0$ is governed by the new Hamiltonian. 1 . Technical details of the solution are given in the following section, which is not absolutely necessary for the understanding of the results presented later in Sec. 5.3.

\subsection{Interaction quench in the Falicov-Kimball model: An- alytical solution}

\subsubsection{Equations of motion in nonequilibrium}

The immobility of the $f$-particles in the Falicov-Kimball model leads to an important simplification for the single-site action Eq. 3.4 in DMFT: the term $\Lambda_{f f}\left(t, t^{\prime}\right)$, which would describe temporal fluctuations of the $f$-particle density, vanishes [47]. The density $f^{\dagger}(t) f(t)$ can be replaced by the time-independent operator $n_{f}$ in the action, and the local Green function 3.6 is thus given by

$$
\begin{aligned}
G\left(t, t^{\prime}\right) & =-i \frac{\operatorname{Tr}_{c, f}\left[e^{-\beta H_{0}} \mathrm{~T}_{\mathcal{C}} \exp \left(S\left[n_{f}\right]\right) c(t) c^{\dagger}\left(t^{\prime}\right)\right]}{\operatorname{Tr}_{c, f}\left[e^{-\beta H_{0}} \mathrm{~T}_{\mathcal{C}} \exp \left(-i S\left[n_{f}\right]\right)\right.} \\
S\left[n_{f}\right] & =-i \int_{\mathcal{C}} d \bar{t} \int_{\mathcal{C}} d \bar{t}^{\prime} c^{\dagger}(\bar{t}) \Lambda\left(\bar{t}, \bar{t}^{\prime}\right) c\left(\bar{t}^{\prime}\right)-i n_{f} \int_{\mathcal{C}} d \bar{t} U(\bar{t}) c^{\dagger}(\bar{t}) c(\bar{t})+\left(E_{f}-\mu\right) n_{f} .
\end{aligned}
$$

for the general case of a time-dependent interaction $U(t)$. In this equation the operators are written in interaction representation with respect to $H_{0}=\mu c^{\dagger} c$. Introducing the partition functions

$$
Z_{n}=\operatorname{Tr}_{c}\left[e^{-\mu c^{\dagger} c} T_{\mathcal{C}} \exp (S[n])\right]
$$

for $n=0,1$, the trace over the $f$ degrees of freedom can be performed, which gives

$$
G\left(t, t^{\prime}\right)=w_{0} Q\left(t, t^{\prime}\right)+w_{1} R\left(t, t^{\prime}\right)
$$

where

$$
\begin{aligned}
& Q\left(t, t^{\prime}\right)=-i \frac{\operatorname{Tr}_{c}\left[e^{-\beta \mu c^{\dagger} c} \mathrm{~T}_{\mathcal{C}} S[0] c(t) c^{\dagger}\left(t^{\prime}\right)\right]}{Z_{0}}, \\
& R\left(t, t^{\prime}\right)=-i \frac{\operatorname{Tr}_{c}\left[e^{-\beta \mu c^{\dagger} c} \mathrm{~T}_{\mathcal{C}} S[1] c(t) c^{\dagger}\left(t^{\prime}\right)\right]}{Z_{1}},
\end{aligned}
$$

\footnotetext{
1 Note that adding a time-dependent single-particle potential term $\epsilon(t) N$ at $t=0$ leads to phase factors $\exp \left[i \int_{t}^{t^{\prime}} d \tau \epsilon(\tau)\right]$ in the Green functions, and thus is of no consequence for equal-time $\left(t=t^{\prime}\right)$ observables. In particular, it is not important whether the time-dependent interaction term is defined by Eq. 3.5, or by $U(t) \sum_{i} n_{i}^{c} n_{i}^{f}$ as we do in the present chapter [cf. Eq. 5.1.
} 
and

$$
w_{1}=1-w_{0}=\frac{Z_{1}}{Z_{0}+Z_{1}}
$$

is the $f$-particle density. These equations were first solved for the equilibrium case by Brandt and Mielsch [47]. In general the $f$-particle density $w_{1}$ depends on the $c$-particle configuration and $E_{f}$ through Eqs. (5.6) and (5.10) in a complicated way and is not known a priori. In particular, it is sublattice-dependent in the checkerboard phase. For nonequilibrium, however, these complications are of minor importance because the $w_{n}$ are time-independent; they can be determined by an equilibrium calculation for the initial state, and hence the inclusion of charge-ordered phases does not add much difficulty to a nonequilibrium calculation. On the other hand, since the charge order parameter is conserved in time, one cannot expect particularly interesting results either. The following calculation is thus restricted to the homogeneous phase, where $w_{1}$ and all local quantities are site-independent, and the $w_{1}$ enter as initial conditions (for the homogeneous case, specification of either $E_{f}$ or $w_{1}$ is equivalent.). For half-filling, we will simply have $w_{1}=$ $w_{0}=\frac{1}{2}$.

The Green functions $(5.8)$ and $(5.9)$ are completely determined by the equations of motion (Sec. 2.3),

$$
\begin{aligned}
{\left[i \partial_{t}+\mu\right] Q\left(t, t^{\prime}\right)-(\Lambda * R)\left(t, t^{\prime}\right) } & =\delta_{\mathcal{C}}\left(t, t^{\prime}\right) \\
{\left[i \partial_{t}+\mu-U(t)\right] R\left(t, t^{\prime}\right)-(\Lambda * R)\left(t, t^{\prime}\right) } & =\delta_{\mathcal{C}}\left(t, t^{\prime}\right)
\end{aligned}
$$

with antiperiodic boundary conditions 2.11 in both arguments on the contour $\mathcal{C}$. From its definition (5.9) it follows that the Green function $R\left(t, t^{\prime}\right)$ has a simple expression in terms of operators,

$$
w_{1} R\left(t, t^{\prime}\right)=-i \frac{\operatorname{Tr}_{c, f}\left[e^{-\beta H_{0}} \mathrm{~T}_{\mathcal{C}} \exp \left(S\left[n_{f}\right]\right) c(t) f^{\dagger}(t) f(t) c^{\dagger}\left(t^{\prime}\right)\right]}{\operatorname{Tr}_{c, f}\left[e^{-\beta H_{0}} \mathrm{~T}_{\mathcal{C}} \exp \left(S\left[n_{f}\right]\right)\right.} \equiv \Gamma\left(t, t^{\prime}\right)
$$

where the right hand side corresponds to the two-particle correlation function 3.9 that arises in the equations of motion for the local Green function and a Hubbard interaction. One can thus directly read off the self-energy from Eq. (3.8),

$$
w_{1} U(t) R\left(t, t^{\prime}\right)=(\Sigma * G)\left(t, t^{\prime}\right)
$$

Several observables of the lattice system (5.1) can be calculated directly from these Green functions analogous to the Hubbard model (cf. Sec. 3.3, compared to Sec. 3.3, one must replace $c_{i \downarrow}$ by $c, c_{i \uparrow}$ by $f$, and set $\left.V_{i j \uparrow}=0 V_{i j \downarrow}=V_{i j}\right)$. In particular, the density $n_{c}$ of the mobile particles, the double occupation $d(t)$, and the internal energy $E$ are obtained 
from the Green functions (5.5a) and 5.12 ,

$$
\begin{aligned}
n_{c} & =\frac{1}{L} \sum_{i}\left\langle\hat{c}_{i}^{\dagger}(t) \hat{c}_{i}(t)\right\rangle=-i G^{<}(t, t), \\
d(t) & =\frac{1}{L} \sum_{i}\left\langle\hat{c}_{i}^{\dagger}(t) \hat{c}_{i}(t) \hat{f}_{i}^{\dagger} \hat{f}_{i}\right\rangle=-i w_{1} R^{<}(t, t), \\
E & =\frac{1}{L} \sum_{i j} t_{i j}\left\langle\hat{c}_{i}^{\dagger}(t) \hat{c}_{j}(t)\right\rangle_{0}+d(t) U(t)=\partial_{t} G^{<}\left(t, t^{\prime}\right)_{t^{\prime}=t}+\mu n_{c},
\end{aligned}
$$

and the momentum distribution (which depends only on the single-particle energy $\epsilon_{\boldsymbol{k}}$ ) is given by

$$
n(\epsilon, t)=\left\langle\hat{c}_{\boldsymbol{k}}^{\dagger}(t) \hat{c}_{\boldsymbol{k}}(t)\right\rangle_{\epsilon_{\boldsymbol{k}}=\epsilon}=-i G_{\epsilon}^{<}(t, t)
$$

where the momentum-resolved Green function is obtained from the Dyson equation,

$$
\left(i \partial_{t}+\mu-\epsilon\right) G_{\epsilon}\left(t, t^{\prime}\right)-\left[\Sigma * G_{\epsilon}\right]\left(t, t^{\prime}\right)=\delta_{\mathcal{C}}\left(t, t^{\prime}\right) .
$$

Together with the self-consistency condition for a semielliptic density of states [cf. Eq. (3.19)],

$$
\Lambda\left(t, t^{\prime}\right)=V^{2} G\left(t, t^{\prime}\right) .
$$

Eqs. (5.7), 5.11a), and (5.11b form a complete set of equations for the local Green functions of the homogeneous phase of the Falicov-Kimball model with time-dependent interaction. External parameters are the chemical potential $\mu$, the average number of $f$-particles $w_{1}$, and the temperature $T$ of the initial state, which enters implicitly through the definition of the contour $\mathcal{C}$. These equations can in general be solved only numerically (Ch. 7). The sudden change of the interaction is a exceptional case in which they can be solved analytically [71, 73] and this solution is now given in detail.

\subsubsection{Langreth rules}

To solve the contour equations (5.11a), (5.11b), (5.13), and (5.18) we first rewrite them in terms of their retarded and lesser components (table 2.2), just as done for Eq. (2.36) in Sec. 2.5. In effect, this means that contour derivatives $C\left(t, t^{\prime}\right)=\partial_{t} A\left(t, t^{\prime}\right)$ are replaced by real-time derivatives,

$$
\begin{aligned}
C^{\mathrm{r}}\left(t, t^{\prime}\right) & =\partial_{t} A^{\mathrm{r}}\left(t, t^{\prime}\right), \\
C^{<}\left(t, t^{\prime}\right) & =\partial_{t} A^{<}\left(t, t^{\prime}\right),
\end{aligned}
$$

and convolutions $C\left(t, t^{\prime}\right)=[A * B]\left(t, t^{\prime}\right)$ of two contour Green functions $A$ and $B$ are expressed in terms of their retarded, advanced, and lesser components according to the Langreth rules (Table 2.4)

$$
\begin{aligned}
& C^{\mathrm{r}}\left(t, t^{\prime}\right)=\int_{t^{\prime}}^{t} d \bar{t} A^{\mathrm{r}}(t, \bar{t}) B^{\mathrm{r}}\left(\bar{t}, t^{\prime}\right) \\
& C^{<}\left(t, t^{\prime}\right)=\int_{-\infty}^{t^{\prime}} d \bar{t} A^{<}(t, \bar{t}) B^{\mathrm{a}}\left(\bar{t}, t^{\prime}\right)+\int_{-\infty}^{t} d \bar{t} A^{\mathrm{r}}(t, \bar{t}) B^{<}\left(\bar{t}, t^{\prime}\right) .
\end{aligned}
$$


The integral boundaries account for the fact that retarded (advanced) Green functions $A^{\mathrm{r}(\mathrm{a})}\left(t, t^{\prime}\right)$ vanish when $t<t^{\prime}\left(t>t^{\prime}\right)$. Furthermore, we shifted $0 \rightarrow-\infty$ in the second equation, such that the convolution extends over the whole axis but contributions from the vertical part at $0-i \tau$ can be dropped. This step is discussed in further detail below.

The contour delta function on the right-hand-side of Eqs. (5.11a), (5.11b), and (5.18) vanishes when the lesser component is taken, and it is replaced by the usual delta function $\delta\left(t-t^{\prime}\right)$ for the retarded components. However, because any retarded function $A^{\mathrm{r}}\left(t, t^{\prime}\right)$ vanishes for $t<t^{\prime}$, retarded equations of motion are only considered for $t>t^{\prime}$, and the initial value at $t=t^{\prime}$ is determined by the weight of the delta function and the derivative operator. In particular,

$$
G_{\boldsymbol{k}}^{\mathrm{r}}(t, t)=R^{\mathrm{r}}(t, t)=Q^{\mathrm{r}}(t, t)=-i
$$

is obtained from Eqs. (5.11a), 5.11b), and (5.18). These conditions follow also directly from the anticommutation relation of creation and annihilation operators.

\subsubsection{Stationary states for $t, t^{\prime}<0$ and $t, t^{\prime} \rightarrow \infty$}

For the interaction quench we treat the equations of motion separately in the four regions where both $t$ and $t^{\prime}$ do not change sign; we introduce additional subscripts + and - which indicate whether the time arguments are greater or less than zero, respectively. Inserting Eqs. (5.20a) and (5.21a) into Eqs. (5.11a) and (5.11b) yields a closed set of equations for $R^{\mathrm{r}}\left(t, t^{\prime}\right)$ and $Q^{\mathrm{r}}\left(t, t^{\prime}\right)$,

$$
\begin{gathered}
\Lambda^{\mathrm{r}}\left(t, t^{\prime}\right)=V^{2}\left[w_{1} R^{\mathrm{r}}\left(t, t^{\prime}\right)+w_{0} Q^{\mathrm{r}}\left(t, t^{\prime}\right)\right], \\
{\left[i \partial_{t}+\mu\right] Q^{\mathrm{r}}\left(t, t^{\prime}\right)=\int_{t^{\prime}}^{t} d \bar{t} \Lambda^{\mathrm{r}}(t, \bar{t}) Q^{\mathrm{r}}\left(\bar{t}, t^{\prime}\right),} \\
{\left[i \partial_{t}+\mu-U(t)\right] R^{\mathrm{r}}\left(t, t^{\prime}\right)=\int_{t^{\prime}}^{t} d \bar{t} \Lambda^{\mathrm{r}}(t, \bar{t}) R^{\mathrm{r}}\left(\bar{t}, t^{\prime}\right),}
\end{gathered}
$$

which must be solved for $t>t^{\prime}$, using the initial condition (5.22). The self-consistency equation 5.19) was used in Eq. (5.23a). Note that in Eq. 5.23), Green functions with both time arguments greater or lesser that zero, i.e., the $(++)$ and $(--)$ components, do not mix with other components. Because $U(t)$ is constant for $t>0$ and $t<0$, respectively, the solutions of Eq. (5.23) are thus translationally invariant in time when both $t$ and $t^{\prime}$ have the same sign, and we make the ansatz

$$
\begin{aligned}
A_{ \pm \pm}^{\mathrm{r}}\left(t, t^{\prime}\right) & =a_{ \pm}^{\mathrm{r}}\left(t-t^{\prime}\right) \\
\tilde{a}_{ \pm}^{\mathrm{r}}(z) & =\int_{0}^{\infty} d s e^{i z s} a_{ \pm}^{\mathrm{r}}(s),
\end{aligned}
$$


for all contour functions $A=G, R, Q, \Lambda, G_{\boldsymbol{k}}$, and $\Sigma$ (with $a=g, r, q, \lambda, g_{\boldsymbol{k}}$, and $\sigma$, respectively). Using this ansatz in Eq. (5.23) we obtain a set of cubic equations,

$$
\begin{aligned}
& \tilde{g}_{ \pm}^{\mathrm{r}}(z)=w_{0} \tilde{q}_{ \pm}^{\mathrm{r}}(z)+w_{1} \tilde{q}_{ \pm}^{\mathrm{r}}(z) \\
& \tilde{q}_{ \pm}^{\mathrm{r}}(z)=\left[z+\mu-V^{2} \tilde{g}_{ \pm}^{\mathrm{r}}(z)\right]^{-1} \\
& \tilde{r}_{ \pm}^{\mathrm{r}}(z)=\left[z+\mu-V^{2} \tilde{g}_{ \pm}^{\mathrm{r}}(z)-U_{ \pm}\right]^{-1}
\end{aligned}
$$

that can be solved analytically. These cubic equations are well-known from the DMFT solution of the Falicov-Kimball model in equilibrium [170]. This is of course expected when both $t$ and $t^{\prime}<0$, because before the quench the system indeed is in an equilibrium state. In a similar way, the retarded $(++)$ and $(--)$ components of $\Sigma$ and $G_{\boldsymbol{k}}$ are obtained from Eq. (5.13) and (5.18),

$$
\begin{aligned}
\tilde{\sigma}_{ \pm}^{\mathrm{r}}(z) & =w_{1} U_{ \pm} \tilde{r}_{ \pm}^{\mathrm{r}}(z) / \tilde{g}_{ \pm}^{\mathrm{r}}(z) \\
\tilde{g}_{\boldsymbol{k} \pm}^{\mathrm{r}}(z) & =\left[z+\mu-\epsilon_{\boldsymbol{k}}-\tilde{\sigma}_{ \pm}^{\mathrm{r}}(z)\right]^{-1} .
\end{aligned}
$$

Furthermore, advanced Green functions are directly related to the retarded ones by symmetry 2.15), so that we have

$$
\begin{aligned}
A_{ \pm \pm}^{\mathrm{a}}\left(t, t^{\prime}\right) & =a_{ \pm}^{\mathrm{a}}\left(t-t^{\prime}\right) \\
\tilde{a}_{ \pm}^{\mathrm{a}}(z) & =\int_{-\infty}^{0} d s e^{i z s} a_{ \pm}^{\mathrm{a}}(s)=\tilde{a}_{ \pm}^{\mathrm{r}}\left(z^{*}\right)^{*} .
\end{aligned}
$$

The lesser Green functions are translationally invariant in time only for both $t$ and $t^{\prime}<$ 0 [(-- ) component], when the system is still in equilibrium. One then has [cf. Eq. 2.18c

$$
\begin{aligned}
A_{--}^{<}\left(t, t^{\prime}\right) & =\int \frac{d \omega}{2 \pi} e^{i \omega\left(t^{\prime}-t\right)} \tilde{a}_{-}^{<}(\omega), \\
\tilde{a}_{-}^{<}(\omega) & =f(\omega)\left[\tilde{a}_{-}^{\mathrm{a}}(\omega)-\tilde{a}_{-}^{\mathrm{r}}(\omega)\right],
\end{aligned}
$$

where $f(\omega)=1 /\left(e^{\omega / T}+1\right)$ is the Fermi function, and $\tilde{a}_{-}^{\mathrm{a}}(\omega)-\tilde{a}_{-}^{\mathrm{r}}(\omega)=-2 i \operatorname{Im} a_{-}^{\mathrm{r}}(\omega)$ is proportional to the spectrum of the equilibrium Green function. Mathematically this follows from the solutions of the equations of motion on the full contour, including the vertical part, and taking into account the antiperiodic boundary conditions. For the quench we use Eq. (5.29) as initial condition for the lesser components. Only then can we then let $0 \rightarrow-\infty$, and disregard the vertical part in the Langreth rule $(5.21 \mathrm{~b})$.

On the other hand, we show below that in the limit where both $t$ and $t^{\prime}$ tend to $\infty$ (but their difference is finite), the lesser $(++)$ components take a form very similar to (5.29),

$$
\begin{aligned}
\lim _{t \rightarrow \infty} A_{++}^{<}(t+s, t) & =\int \frac{d \omega}{2 \pi} e^{-i \omega s} \tilde{a}_{+}^{<}(\omega), \\
\tilde{a}_{+}^{<}(\omega) & =F(\omega)\left[\tilde{a}_{+}^{\mathrm{a}}(\omega)-\tilde{a}_{+}^{\mathrm{r}}(\omega)\right] .
\end{aligned}
$$


The function $F(\omega)$ is common for all $a=g, r, q, \sigma$, and $g_{\boldsymbol{k}}$. One can in fact directly see from the equations of motion (5.11a), (5.11b), (5.13), and (5.18) for the lesser component that if the stationary limit (5.30a) exists, then Green functions $\tilde{a}_{+}^{<}(\omega)$ must have this common factor $F(\omega)$. To find this factor, however, the equations of motion must be solved, because it contains the entire information about the initial state.

\subsubsection{Double Fourier transforms}

We now consider the cases with one or two positive time arguments, i.e., after the quench. We introduce double Fourier transforms

$$
\begin{aligned}
& \tilde{A}_{+-}^{\mathrm{r}}(z, \eta)=\int_{0}^{\infty} d t e^{i z t} \int_{-\infty}^{0} d t^{\prime} e^{i \eta t^{\prime}} A_{+-}^{\mathrm{r}}\left(t, t^{\prime}\right), \\
& \tilde{A}_{-+}^{\mathrm{a}}(\eta, z)=\int_{0}^{\infty} d t e^{i z t} \int_{-\infty}^{0} d t^{\prime} e^{i \eta t^{\prime}} A_{-+}^{\mathrm{a}}\left(t^{\prime}, t\right),
\end{aligned}
$$

for retarded and advanced components,

$$
\begin{aligned}
& \int \frac{d \omega}{2 \pi} e^{-i \omega t^{\prime}} \tilde{A}_{+-}^{<}(z, \omega)=\int_{0}^{\infty} d t e^{i z t} A_{+-}^{<}\left(t, t^{\prime}\right) \\
& \int \frac{d \omega}{2 \pi} e^{-i \omega t^{\prime}} \tilde{A}_{-+}^{<}(\omega, z)=\int_{0}^{\infty} d t e^{i z t} A_{-+}^{<}\left(t^{\prime}, t\right)
\end{aligned}
$$

for the lesser components with mixed time arguments (which holds for $t^{\prime}<0$ ), and

$$
\tilde{A}_{++}^{<}(z, \eta)=\int_{0}^{\infty} d t e^{i z t} \int_{0}^{\infty} d t^{\prime} e^{i \eta t^{\prime}} A_{++}^{<}\left(t, t^{\prime}\right)
$$

for the lesser Green function with both time arguments after the quench. In this subsection explicit expressions for $\tilde{A}_{+-}^{\mathrm{r}}(z, \omega), \tilde{A}_{+-}^{<}(z, \omega)$, and $\tilde{A}_{++}^{<}(z, \omega)$ are derived. The remaining functions are then obtained by symmetry 2.15 ,

$$
\begin{aligned}
& \tilde{A}_{-+}^{\mathrm{a}}(\eta, z)=\tilde{A}_{+-}^{\mathrm{r}}\left(-\eta^{*},-z^{*}\right)^{*} \\
& \tilde{A}_{-+}^{<}(\omega, z)=-\tilde{A}_{+-}^{<}\left(-z^{*},-\omega\right)^{*} .
\end{aligned}
$$

Using Langreth rules 5.21 once again yields for the convolutions $C=A * B$

$$
\begin{aligned}
\tilde{C}_{+-}^{\mathrm{r}}(z,-\omega) & =\tilde{A}_{+-}^{\mathrm{r}}(z,-\omega) \tilde{b}_{-}^{\mathrm{r}}(\omega)+\tilde{a}_{+}^{\mathrm{r}}(z) \tilde{B}_{+-}^{\mathrm{r}}(z,-\omega), \\
\tilde{C}_{+-}^{<}(z,-\omega) & =\tilde{A}_{+-}^{<}(z,-\omega) \tilde{b}_{-}^{\mathrm{a}}(\omega)+\tilde{a}_{+}^{\mathrm{r}}(z) \tilde{B}_{+-}^{<}(z,-\omega)+\tilde{A}_{+-}^{\mathrm{r}}(z,-\omega) \tilde{b}_{-}^{<}(\omega), \\
\tilde{C}_{++}^{<}(z, \eta) & =\tilde{A}_{++}^{<}(z, \eta) \tilde{b}_{+}^{\mathrm{a}}(-\eta)+\tilde{a}_{+}^{\mathrm{r}}(z) \tilde{B}_{++}^{<}(z, \eta) \\
& +\int \frac{d \omega}{2 \pi}\left[\tilde{A}_{+-}^{<}(z,-\omega) \tilde{B}_{-+}^{\mathrm{a}}(\omega, \eta)+\tilde{A}_{+-}^{\mathrm{r}}(z,-\omega) \tilde{B}_{-+}^{<}(\omega, \eta)\right] .
\end{aligned}
$$


Furthermore, the derivative $C=\partial_{t}^{\mathcal{C}} A\left(t, t^{\prime}\right)$ translates into

$$
\begin{aligned}
& \tilde{C}_{+-}^{\mathrm{r}}(z, \eta)=z \tilde{A}^{\mathrm{r}}(z, \eta)-i \tilde{a}_{-}^{\mathrm{r}}(-\eta), \\
& \tilde{C}_{+-}^{<}(z, \omega)=z \tilde{A}_{+-}^{<}(z,-\omega)-i \tilde{a}_{-}^{<}(\omega), \\
& \tilde{C}_{++}^{<}(z, \eta)=z \tilde{A}_{++}^{<}(z, \eta)-i \int \frac{d \omega}{2 \pi} \tilde{A}_{-+}^{<}(\omega, \eta),
\end{aligned}
$$

where one must use the continuity of the components at the boundary $t=0$ and $t^{\prime}=0$, e.g., $A_{+-}^{\mathrm{r}}\left(0, t^{\prime}\right)=A_{--}^{\mathrm{r}}\left(0, t^{\prime}\right)$.

Using Eqs. (5.35) and (5.36) one can rewrite Eq. (5.11) for the various components and solve them using the self-consistency equation (5.19). For instance, we obtain the $(+-)$ component of the retarded Green functions as

$$
\begin{aligned}
& \tilde{R}_{+-}^{\mathrm{r}}(z, \eta)=\left[\tilde{\Lambda}_{+-}^{\mathrm{r}}(z, \eta)+i\right] r_{+}^{\mathrm{r}}(z) r_{-}^{\mathrm{r}}(-\eta), \\
& \tilde{Q}_{+-}^{\mathrm{r}}(z, \eta)=\left[\tilde{\Lambda}_{+-}^{\mathrm{r}}(z, \eta)+i\right] q_{+}^{\mathrm{r}}(z) q_{-}^{\mathrm{r}}(-\eta),
\end{aligned}
$$

where Eq. (5.25) was used once. Together with the self-consistency (5.19), this is a simple linear equation for $\Lambda_{+-}^{\mathrm{r}}(z, \eta)$. In a similar way all components are determined successively: Starting from the retarded $(+-)$ and advanced $(-+)$ components, the results enter the lesser $(+-)$ and lesser $(-+)$ components [cf. Eqs. (5.35b) and (5.36b)], which in turn enter the equations for the lesser $(++)$ component [cf. Eqs. (5.35c) and (5.36c)]. The procedure is repeated for Eq. (5.11), Eq. (5.13), and finally for the lattice Dyson equation (5.18), which yields the momentum-dependent Green function $G_{\boldsymbol{k}}\left(t, t^{\prime}\right)$.

For completeness we state the final result for $G, R$, and $G_{\boldsymbol{k}}$. For this we introduce the abbreviations

$$
\begin{aligned}
M_{\alpha \beta}^{x y} & =\left[1-V^{2}\left(w_{1} \tilde{r}_{\alpha}^{x} \tilde{r}_{\beta}^{y}+w_{0} \tilde{q}_{\alpha}^{x} \tilde{q}_{\beta}^{y}\right)\right]^{-1}, \\
\kappa_{\alpha}^{x} & =\tilde{r}_{\alpha}^{x} \tilde{q}_{\alpha}^{x} / \tilde{g}_{\alpha}^{x}, \\
K_{\alpha \beta}^{x y} & =1+w_{1} w_{0} U_{+} U_{-} \kappa_{\alpha}^{x} \kappa_{\beta}^{y} M_{\alpha \beta}^{x y} .
\end{aligned}
$$

with superscripts $x, y \in\{\mathrm{r}, \mathrm{a}\}$, and subscripts $\alpha, \beta \in\{+,-\}$, and we use the convention that the variables of a function $a_{\alpha}^{x}$ is (i) $z$ when $x=\mathrm{r}$ and $\alpha=+$, (ii) $-\eta$ when $x=\mathrm{a}$ and $\alpha=+$, and (iii) $\omega$ when $\alpha=-$. In terms of these expressions the final result is

$$
\begin{aligned}
\tilde{G}_{+-}^{\mathrm{r}}(z,-\omega) & =i V^{-2}\left(M_{+-}^{\mathrm{rr}}-1\right), \\
\tilde{R}_{+-}^{\mathrm{r}}(z,-\omega) & =i \tilde{r}_{+}^{\mathrm{r}} \tilde{r}_{-}^{\mathrm{r}} M_{+-}^{\mathrm{rr}}, \\
\tilde{G}_{\boldsymbol{k}+-}^{\mathrm{r}}(z,-\omega) & =i \tilde{g}_{\boldsymbol{k}+}^{\mathrm{r}} \tilde{g}_{\boldsymbol{k}-}^{\mathrm{r}} K_{+-}^{\mathrm{rr}},
\end{aligned}
$$

and

$$
\begin{aligned}
\tilde{G}_{+-}^{<}(z,-\omega) & =i f(\omega)\left(M_{+-}^{\mathrm{ra}}-M_{+-}^{\mathrm{rr}}\right) / V^{2}, \\
\tilde{R}_{+-}^{<}(z,-\omega) & =i f(\omega) \tilde{r}_{+}^{\mathrm{r}}\left(\tilde{r}_{-}^{\mathrm{a}} M_{+-}^{\mathrm{ra}}-\tilde{r}_{-}^{\mathrm{r}} M_{+-}^{\mathrm{rr}}\right), \\
\tilde{G}_{\boldsymbol{k}+-}^{<}(z,-\omega) & =i f(\omega) \tilde{g}_{\boldsymbol{k}+}^{\mathrm{r}}\left(\tilde{g}_{\boldsymbol{k}-}^{\mathrm{a}} K_{+-}^{\mathrm{ra}}-\tilde{g}_{\boldsymbol{k}-}^{\mathrm{r}} K_{+-}^{\mathrm{rr}}\right) .
\end{aligned}
$$




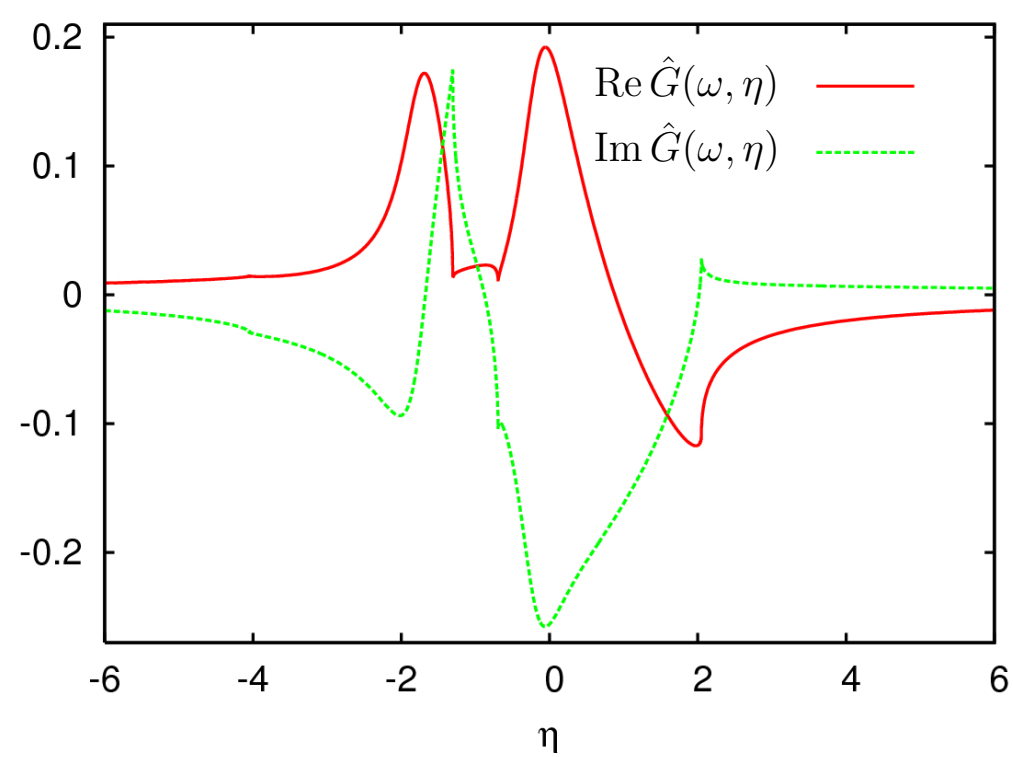

Figure 5.2: The function $\hat{G}(\omega, \eta)$ that must be Fourier-transformed in order to get $G(\omega, t)$, Eq. (5.47); $\omega=0.125$ is chosen arbitrary.

The lesser $(++)$ component can be written in the form

$$
A_{++}^{<}(z, \eta)=\tilde{F}(z, \eta) \frac{\tilde{a}_{+}^{\mathrm{a}}-\tilde{a}_{+}^{\mathrm{r}}}{\eta+z}+F_{A}(z, \eta)
$$

for $A=G, R$, and $G_{\boldsymbol{k}}$, where

$$
\tilde{F}(z, \eta)=\int \frac{d \omega}{2 \pi} f(\omega) \frac{M_{+-}^{\mathrm{rr}}+M_{+-}^{\mathrm{aa}}-M_{+-}^{\mathrm{ra}}-M_{+-}^{\mathrm{ar}}}{z+\eta+V^{2}\left(\tilde{g}_{+}^{\mathrm{a}}-\tilde{g}_{+}^{\mathrm{r}}\right)},
$$

and

$$
\begin{aligned}
& F_{G}(z, \eta)=\tilde{F}(z, \eta) / V^{2} \\
& F_{R}(z, \eta)=\tilde{r}_{+}^{\mathrm{r}} \tilde{r}_{+}^{\mathrm{a}} \int \frac{d \omega}{2 \pi} f(\omega)\left(\tilde{r}_{-}^{\mathrm{a}} M_{+-}^{\mathrm{aa}} M_{+-}^{\mathrm{ra}}-\tilde{r}_{-}^{\mathrm{r}} M_{+-}^{\mathrm{rr}} M_{+-}^{\mathrm{ar}}\right) \\
& F_{G_{k}}(z, \eta)=\tilde{g}_{\boldsymbol{k}+}^{\mathrm{r}} \tilde{g}_{\boldsymbol{k}+}^{\mathrm{a}}\left\{-\tilde{F}(z, \eta)+\int \frac{d \omega}{2 \pi} f(\omega)\right. \\
& \times\left[w_{0} w_{1} U_{+}^{2} \tilde{\kappa}_{+}^{\mathrm{r}} \tilde{\kappa}_{+}^{\mathrm{a}}\left(\tilde{\kappa}_{-}^{\mathrm{a}} M_{+-}^{\mathrm{aa}} M_{+-}^{\mathrm{ra}}-\tilde{\kappa}_{-}^{\mathrm{r}} M_{+-}^{\mathrm{rr}} M_{+-}^{\mathrm{ar}}\right)\right. \\
&\left.\left.+\tilde{g}_{\boldsymbol{k}-}^{\mathrm{a}} K_{+-}^{\mathrm{aa}} K_{+-}^{\mathrm{ra}}-\tilde{g}_{\boldsymbol{k}-}^{\mathrm{r}} K_{+-}^{\mathrm{rr}} K_{+-}^{\mathrm{ar}}\right]\right\} .
\end{aligned}
$$

\subsubsection{Back transformation}

To obtain the physical real-time Green functions, we have to invert the double Fourier transformations (5.31), (5.32), and (5.33), using the final expressions (5.39) through 
5.42). Here we give an explicit formula for the partially Fourier-transformed lesser component

$$
\tilde{A}^{<}(\omega, t)=\int d s e^{i \omega s} A^{<}(t+s, t)
$$

$\left(A=G, R\right.$, and $\left.G_{\boldsymbol{k}}\right)$. The singularity at $\eta+z=0$ in Eq. (5.42a) determines 5.43$)$ in the limit $t \rightarrow \infty$,

$$
\tilde{a}_{+}^{<}(\omega) \equiv \lim _{t \rightarrow \infty} \tilde{A}^{<}(\omega, t)=\left[\tilde{a}_{+}^{\mathrm{a}}(\omega)-\tilde{a}_{+}^{\mathrm{r}}(\omega)\right] \operatorname{Im} \tilde{F}(\omega,-\omega)
$$

which is of the form discussed above [cf. Eq. [5.30]], with

$$
\begin{aligned}
& F(\omega)=\operatorname{Im} \tilde{F}(\omega,-\omega)=\int d \omega^{\prime} T\left(\omega, \omega^{\prime}\right) f\left(\omega^{\prime}\right) \\
& T\left(\omega, \omega^{\prime}\right)=\frac{1}{2 \pi \operatorname{Im} \tilde{g}_{+}^{\mathrm{r}}(\omega)} \operatorname{Re}\left\{\frac{1}{1-V^{2}\left[w_{1} \tilde{r}_{+}^{r}(\omega) \tilde{r}_{-}^{r}\left(\omega^{\prime}\right)+w_{0} \tilde{q}_{+}^{r}(\omega) \tilde{q}_{-}^{r}\left(\omega^{\prime}\right)\right]}-\right. \\
&\left.-\frac{1}{1-V^{2}\left[w_{1} \tilde{r}_{+}^{r}(\omega) \tilde{r}_{-}^{a}\left(\omega^{\prime}\right)+w_{0} \tilde{q}_{+}^{r}(\omega) \tilde{q}_{-}^{a}\left(\omega^{\prime}\right)\right]}\right\},
\end{aligned}
$$

where $\tilde{F}(z, \eta)$ in the first line is given by Eq. $(5.42 \mathrm{~b})$, and in the second line we have explicitly inserted expression (5.39a) (replacing $z=-\eta \rightarrow \omega$ and $\omega \rightarrow \omega^{\prime}$ ) and used the symmetry $(5.28 \mathrm{~b})$, which implies $M_{+-}^{\mathrm{rr}}=\left(M_{+-}^{\mathrm{aa}}\right)^{*}$ and $M_{+-}^{\mathrm{ra}}=\left(M_{+-}^{\mathrm{ra}}\right)^{*}$ for $z=-\eta$. The matrix $T\left(\omega, \omega^{\prime}\right)$ plays the role of a transfer matrix from the Fermi function $f(\omega)$ of the initial state to the effective Fermi function $F(\omega)$ after relaxation. The full result for $\tilde{A}^{<}(\omega, t)$ is thus given by

$$
\tilde{A}^{<}(\omega, t)=\sum_{ \pm} \Theta( \pm t)\left[\tilde{a}_{ \pm}^{<}(\omega)+e^{-i \omega t} \int \frac{d \eta}{2 \pi} e^{-i t \eta} \hat{A}_{ \pm}(\omega, \eta)\right]
$$

where

$$
\begin{aligned}
& \hat{A}_{-}(\omega, \eta)=\tilde{A}_{+-}^{<}(\omega, \eta)+\frac{\tilde{a}_{-}^{<}(-\eta)-\tilde{a}_{-}^{<}(\omega)}{i(\eta+\omega)} \\
& \hat{A}_{+}(\omega, \eta)=2 i \operatorname{Im} \frac{\tilde{F}(\omega, \eta)\left[\tilde{a}_{+}^{\mathrm{a}}(-\eta)-\tilde{a}_{+}^{\mathrm{r}}(\omega)\right]}{\omega+\eta}+F_{A}(\omega, \eta)+\int \frac{d \omega^{\prime}}{2 \pi i} \frac{\tilde{A}_{-+}^{<}\left(\omega^{\prime}, \eta\right)}{\omega-i 0-\omega^{\prime}} .
\end{aligned}
$$

The components for $A=G, R$, and $G_{\boldsymbol{k}}$ were given in the previous subsection. Note that the first term on the right hand side of Eq. (5.47c) is regular at $\eta=-\omega$, because both $\tilde{F}(\omega, \eta)$ and $\tilde{a}_{+}^{\mathrm{a}}(-\eta)-\tilde{a}_{+}^{\mathrm{r}}(\omega)$ are then purely imaginary.

The evaluation of the Fourier integrals (5.47) is done numerically. This is straightforward to do in principle, but care has to be taken because (i) the functions $(5.47 \mathrm{~b})$ and (5.47k) have power law tails for $|\eta| \rightarrow \infty$, and (ii) they have a number of kinks with square-root divergences in the derivatives (see Fig. 5.2). In the numerical evaluation, the tails are fitted by inverse power laws up to fourth degree $1 / \eta^{4}$ and integrated exactly. 


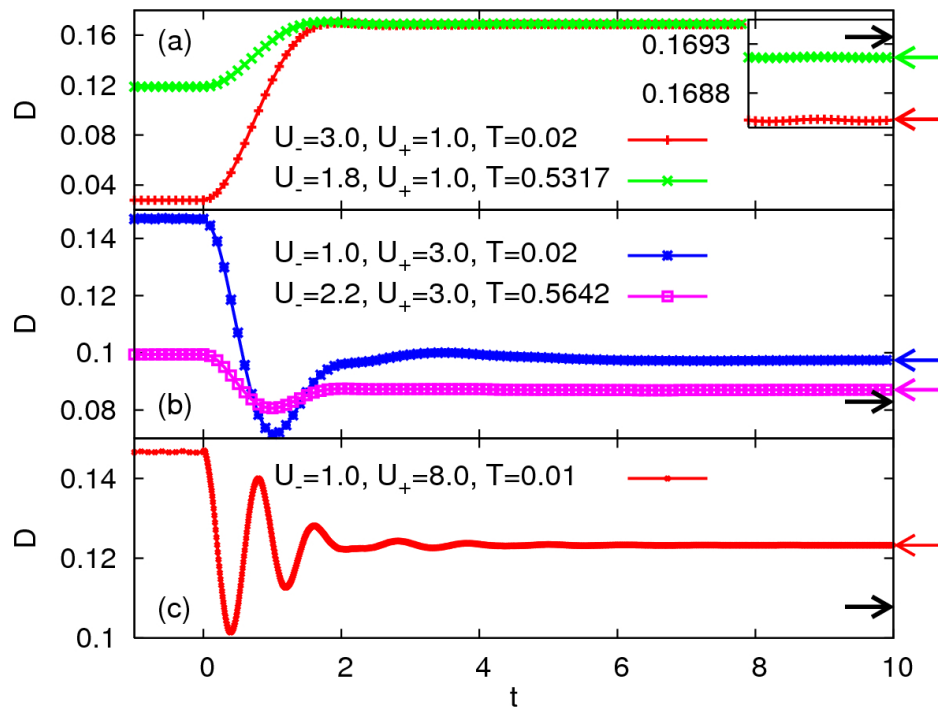

Figure 5.3: (Reprinted from Ref. [71]) Double occupation $D(t)$ for quenches to (a) $U_{+}=$ 1, (b) $U_{+}=3$, and (c) $U_{+}=8$, starting from an initial metallic $\left(U_{-}<2\right)$ or insulating state $\left(U_{-}>2\right)$. The half-bandwidth is $2 V \equiv 2$. In (a) and (b), the internal energy is the same after both quenches. Thick right-pointing arrows mark the double occupation in the thermal state for interaction $U_{+}$with the same density and internal energy. These values differ from the stationary value $D_{\infty}$, marked by left-pointing arrows, which are approached for large times. The inset in (a) shows a magnification of the large- $t$ behavior.

The kinks are handled by a suitable adaptive $\eta$-grid. Note that the numerical effort is quite large for an "analytical" solution. It turns out that the numerical integration of the equations of motion that was later implemented for the investigation of the adiabatic behavior (Ch. 7) is faster and more accurate at small times than the present method (the results of both methods agree). However, the long-time limit is accessible only in the analytical solution through the single integral (5.46) over a bounded function on a compact interval.

\subsection{Results: Non-thermal steady states}

\subsubsection{Time evolution of the double occupation}

In the following section we present results for an interaction quench in the homogeneous phase of the Falicov-Kimball model at half-filling for both $c$ and $f$ electrons $\left(n_{c}=n_{f}=\frac{1}{2}\right)$. For these parameters, and a semielliptic density of states with quarter bandwidth $V=1$, the metal-insulator transition occurs at the critical interaction $U_{c}=2$. In Fig. 5.3 the double occupation $d(t)$ is plotted for various quenches within and between the two phases. In all cases $d(t)$ relaxes to a new stationary value $D_{\infty}$ on the time scale $1 / V$. When the final interaction $U_{+}$is small, the relaxation is almost monotonic (Fig. $5.3 \mathrm{a}$ ), while damped oscillations arise after quenches to large interactions $\left(U_{+}>V\right)$ (Fig. 5.3c). As discussed 


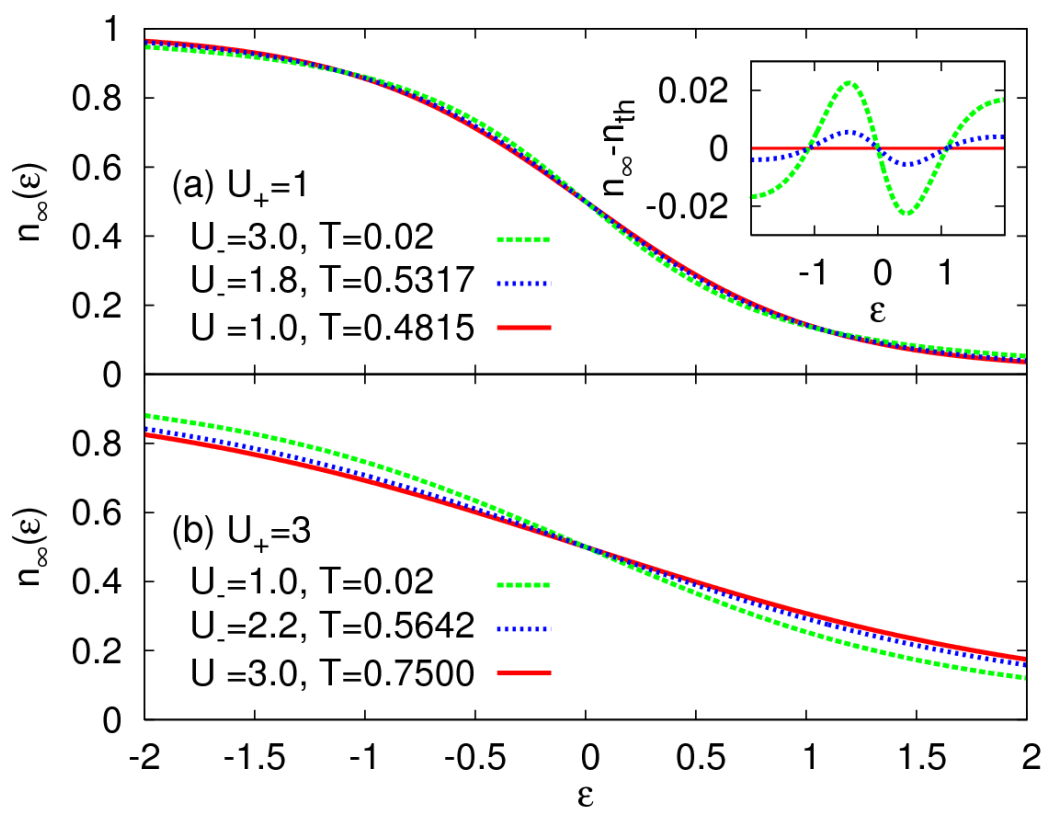

Figure 5.4: (Reprinted from Ref. [71]) Momentum distribution (5.50) for the same quench as in Fig. 5.3. The solid line is the thermal expectation for the indicated temperature $T_{*}$.

in Sec. 4.4, such transient oscillations are related to the collapse and revival oscillations that occur in the atomic limit, and are expected to prevail in the strong-coupling limit $V \ll U$. A detailed comparison of the exact results to the strong-coupling results from Sec. 4.4 will be made in Sec. 5.3.4.

\subsubsection{The steady state}

The limit $t \rightarrow \infty$ can be directly computed for the expectation values of various observables from the expressions of the last section. Without going into the details let us briefly repeat the mathematical structure of these expressions: in equilibrium, the lesser Green functions which determine various expectation values [cf. Eq. (5.14)-(5.17)] is given by a product of the spectrum of the retarded Green function and the Fermi function [cf. Eq. (2.18c)]. A similar relation holds in the long-time limit after a quench in the FalicovKimball for the Fourier transform (5.44) of the lesser Green functions [cf. Eq. (5.30)]. The spectra are the same as in equilibrium, but the Fermi function is replaced by a nonthermal distribution function or effective Fermi function $F(\omega)$, which is related to the initial state Fermi function by a transfer matrix $T\left(\omega, \omega^{\prime}\right)$ [cf. Eq. (5.45)]. The latter depends only the temperature-independent spectra at $U=U_{+}$and $U=U_{-}$, [cf. Eq. (5.46)].

This simple factorization is certainly special for the homogeneous phase of the FalicovKimball model. In general, the spectrum of a correlated system depends on temperature, i.e., on the state of the system. One can therefore not expect that spectra in the nonequilibrium state only depend on the parameters of the Hamiltonian either. For the FalicovKimball model, however, all memory on the initial state is entailed in the single distri- 
bution function $F(\omega)$. Using the factorization (5.44), and the definition of the retarded equilibrium functions [Eqs. (5.25) - (5.27)], one obtains the direct expression long-time limit for several observables (5.14) - 5.17),

$$
\begin{aligned}
n_{c, \infty} & =\lim _{t \rightarrow \infty} n_{c}(t)=-w_{1} \int \frac{d \omega}{\pi} F(\omega) \operatorname{Im} \tilde{g}_{+}^{\mathrm{r}}(\omega) \\
d_{\infty} & =\lim _{t \rightarrow \infty} d(t)=-w_{1} \int \frac{d \omega}{\pi} F(\omega) \operatorname{Im} \tilde{r}_{+}^{\mathrm{r}}(\omega) \\
n_{\infty}(\epsilon) & =\lim _{t \rightarrow \infty} n(\epsilon, t)=-\int \frac{d \omega}{\pi} F(\omega) \operatorname{Im} \tilde{g}_{\epsilon+}^{\mathrm{r}}(\omega) \\
E_{\infty} & =E(t)=-\int \frac{d \omega}{\pi} F(\omega) \omega \operatorname{Im} \tilde{g}_{+}^{\mathrm{r}}(\omega)+\mu n_{c,} .
\end{aligned}
$$

The internal energy and the $c$-particle density are constant for all times $t>0$ due to energy and particle number conservation. To test for thermalization, the long-time expectation values (5.49) and (5.50) are compared to the corresponding equilibrium expectation values in a grand-canonical ensemble with the same total energy and $c$-particle density [cf. Eq. (4.8)], i.e., the effective temperature $T_{*}$ and the chemical potential $\delta \mu_{*}$ are chosen such that one has $E_{\infty}=E^{t h}$ and $n_{c, \infty}=n_{c}^{t h}$. The thermal expectation values $d^{t h}, n^{t h}(\epsilon), n_{c}^{t h}$, and $E^{\text {th }}$ are given by the same expression as the long-time values [Eq. (5.49)-(5.51)], only replacing the distribution $F(\omega)$ by the Fermi function $f\left(\omega-\mu_{*}\right)$ with effective temperature $T_{*}$.

The stationary value $d_{\infty}$ is clearly not the same as the double occupation in the thermal state with the same density and internal energy (thick arrows in Fig. $5.3 \mathrm{a}$ and $5.3 \mathrm{~b})$. Analogous deviations to the thermal state are also observed for the occupation $n(\epsilon, t)$ of single-particle states (Fig. 5.4). The initial temperature for each pair of quenches shown in Figs. $5.3 \mathrm{a}, 5.3 \mathrm{~b}, 5.4 \mathrm{a}, 5.4 \mathrm{~b}$, respectively, is chosen such that the final energy $E(t$ $>0)$ is the same for both quenches in each figure. The differences in the distribution and the double occupation for those two quenches are thus another way to access the failure of the thermal ensemble, which depends only on the total energy, particle number, and interaction $U 2$

Another way to identify the nonthermal behavior for the Falicov-Kimball model is by means of the deviations of the nonthermal distribution $F(\omega)$ to the Fermi function. In Fig. $5.5 \mathrm{a}, F(\omega)$ is plotted for quenches from $U_{-}=1(T=0)$ to various values of $U+$. When the quench amplitude $U_{+}-U_{-}$is large, $F(\omega)$ becomes a nonmonotonous function and clearly cannot be fitted by a Fermi function of any temperature.

\section{Infinitesimal quenches}

In the Falicov-Kimball model, thermalization does not even occur for an infinitesimal interaction quench $\delta U=U_{+}-U_{-} \rightarrow 0$ and infinite waiting time. This is discussed in the following for the two cases when either (i) the initial state is $T=0$, and (ii) when $T>0$.

\footnotetext{
${ }^{2}$ After these results were published in Ref. 71, another (purely numerical) study showed that the Falicov-Kimball model keeps some memory on the initial state over long times after a perturbation [178].
} 

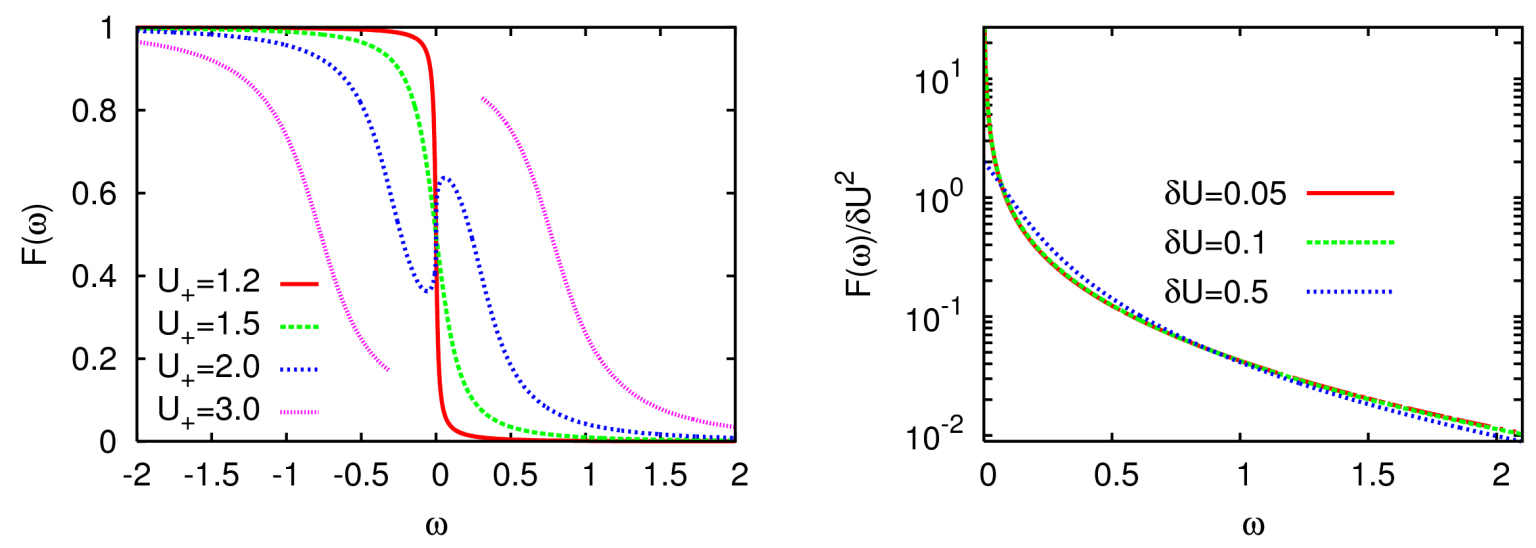

Figure 5.5: Left panel: The nonthermal distribution $F(\omega)$ [Eq. (5.46)] for quenches from $U_{-}=1(T=0)$ to various $U_{+}$, as indicated. For $U_{+}=3$, the spectrum is gapped, and $F(\omega)$ is only plotted outside the gap. Right panel: $F(\omega) / \delta U^{2}$ for $\omega>0$, for quenches from $U_{-}=1(T=0)$ to $U_{+}=U_{-}+\delta U$, plotted on a logarithmic scale.

(i) For a small quench, $F(\omega)$ can be expanded in $\delta U$. When the initial state is at $T$ $=0$, differences to the thermal state appear in second order in $\delta U$ : Numerically, one can see that $F(\omega, \delta U) / \delta U^{2}$ has a finite value in the limit $\delta U \rightarrow 0$ for an interaction quench starting and $T=0$ (Fig. 5.5b). In contrast, thermalization for small quenches would imply that $F(\omega) \sim\left[e^{\beta_{*}(\delta U)\left[\omega-\mu_{*}(\delta U)\right]}+1\right]^{-1}$ with $\beta_{*}(\delta U) \rightarrow \infty$ and $\mu_{*}(\delta U) \rightarrow 0$ for $\delta U \rightarrow$ 0 , and hence $\log F(\omega, \delta U) /\left(\delta U^{2}\right)$ would diverge for fixed $\omega$ and $\delta U \rightarrow 0$.

(ii) When the initial state is at finite temperature, the difference of the final state and a thermal state turn out to be first order in $\delta U$, and thus are accessible more easily from the exact result. For this purpose we evaluate the derivative $\partial F(\omega) / \partial U_{+}$at $U_{+}=U_{-}$ from the exact result (5.46) and the cubic equations for the Green functions (5.25). After some calculation this gives

$$
\left.\frac{\partial F(\omega)}{\partial U_{+}}\right|_{U_{+}=U_{-}}=-w_{1} \frac{\operatorname{Im} \tilde{r}_{+}^{\mathrm{r}}(\omega)}{\operatorname{Im} \tilde{g}_{+}^{\mathrm{r}}(\omega)} \frac{\partial f(\omega)}{\partial \omega} .
$$

On the other hand, if one assumes thermalization for $\delta U \rightarrow 0$ one would have

$$
\left.F(\omega) \sim \frac{1}{e^{\beta_{*}(\delta U)\left[\omega-\mu_{*}(\delta U)\right]+1}} \Rightarrow \frac{\partial F(\omega)}{\partial U_{+}}\right|_{U_{+}=U_{-}}=\frac{\partial f(\omega)}{\partial \omega}\left[\frac{d \beta}{d U_{+}} \frac{\omega}{\beta_{*}}-\frac{d \mu_{*}}{d U_{+}}\right],
$$

which clearly cannot be the same as $(5.52)$ in general, because the pre-factor of $\partial f(\omega) / \partial \omega$ in (5.52) contains the ratio of two spectra (5.25) which is not a linear function of $\omega$ for general $U$.

\subsubsection{Discussion}

The nonthermal behavior of the Falicov-Kimball model is closely related to the immobility of the $f$-particles. Since the time evolution preserves the weight of each $f$-particle 
configuration $\left\{n_{i}^{f}\right\}$, the most general ansatz for a stationary state density matrix reads

$$
\rho_{\infty}=\sum_{\left\{n_{i}^{f}\right\}} P_{\left\{n_{i}^{f}\right\}} W\left[\left\{n_{i}^{f}\right\}\right] \rho_{\infty}\left[\left\{n_{i}^{f}\right\}\right]
$$

where the weights $W\left[\left\{n_{i}^{f}\right\}\right]$ are the same as in the initial state $(5.4)$, while the density matrix $\rho_{\infty}\left[\left\{n_{i}^{f}\right\}\right]$ of the $c$-particles has yet to be determined. It is clear that 5.54 is different from the annealed disorder state (5.2), in which the weights would depend on the new c-particle configuration. However, thermalization never means that the density matrix itself equals the Gibbs ensemble, but only that it may be replaced by the latter one for the sake of computing expectation values in some class of observables, e.g., local observables in the thermodynamic limit (Ch. 4). In the following we will therefore not attempt to show from (5.54) that the long-time limit must have detailed memory on the initial state, but rather try to find which information on the initial state has to be included at least in the density matrix (5.54) in order to describe the final state properly. This will then give an understanding of the physics that leads to the nonthermal behavior.

To begin with, one might assume that the essential memory on the initial state is in the weights $W\left[\left\{n_{i}^{f}\right\}\right]$ in $(5.4)$, and that the final state is therefore already given by $(5.54)$ when $\rho_{\infty}\left[\left\{n_{i}^{f}\right\}\right]$ is replaced by the canonical ensemble. This is certainly not true for the quench in the homogeneous phase of the Falicov-Kimball model in $d=\infty$ for the following reason: As explained below Eq. (5.4), the DMFT equations for the homogeneous phase are equivalent to CPA for quenched disorder, where all weights $W\left[\left\{n_{i}^{f}\right\}\right]$ are equal and hence cannot carry any memory on the initial state at all. However, even if one assumes that each individual $c$-particle configuration can be replaced by a thermal one, the effective temperature $T_{*}$ would depend on the $f$-particle configuration $\left\{n_{i}^{f}\right\}$ because the energy that is put into the system during the quench explicitly depends on $\left\{n_{i}^{f}\right\}$. To what extent the corresponding temperature fluctuations in Eq. (5.54), with $\rho_{\infty}\left[\left\{n_{i}^{f}\right\}\right]$ replaced by $e^{-H_{c}\left[\left\{n_{i}^{f}\right\}\right] / T_{*}\left[\left\{n_{i}^{f}\right\}\right]} / Z$, entail detailed memory on the initial state in the thermodynamic limit is an interesting question that cannot be answered here.

However, the explanation of the nonthermal behavior is much simpler: The $c$-particle Hamiltonian $H_{c}\left[\left\{n_{i}^{f}\right\}\right]$ for given $\left\{n_{i}^{f}\right\}$ and $U=U_{+}$is quadratic, and quadratic Hamiltonians do in general not lead to thermalization (Sec. 4.3). More precisely, $H_{c}$ can be diagonalized in the form

$$
H_{c}=\sum_{\alpha} \epsilon_{\alpha} c_{\alpha}^{\dagger} c_{\alpha}
$$

where $|\alpha\rangle$ and $\epsilon_{\alpha}$ are single-particle eigenstates and eigenvalues of the single-particle Hamiltonian

$$
h_{c}=V \sum_{i j} t_{i j}|i\rangle\left\langle j\left|+U_{+} \mathcal{F}, \quad \mathcal{F}=\sum_{i}\right| i\right\rangle n_{i}^{f}\langle i|,
$$

respectively, and $c_{\alpha}=\sum_{i}\langle\alpha \mid i\rangle c_{i}$. To simplify the notation, the dependence of $h_{c},|\alpha\rangle$, and $\epsilon_{\alpha}$ on $\left\{n_{i}^{f}\right\}$ was omitted. Following the discussion in Sec. 4.3 one may thus conjecture 
that the final state is given by 5.54 when $\rho_{\infty}\left[\left\{n_{i}^{f}\right\}\right]$ is replaced by the Gibbs ensemble for each $\left\{n_{i}^{f}\right\}$

$$
\rho_{\infty}\left[\left\{n_{i}^{f}\right\}\right]=\rho_{\mathrm{G}} \equiv \frac{\exp \left(\sum_{\alpha} \lambda_{\alpha} n_{\alpha}\right)}{\operatorname{Tr} \exp \left(\sum_{\alpha} \lambda_{\alpha} n_{\alpha}\right)}
$$

with constraints

$$
\operatorname{Tr}\left[\rho_{\mathrm{G}} n_{\alpha}\right]=\operatorname{Tr}\left[\rho_{0} n_{\alpha}\right]
$$

where $\rho_{0} \propto \exp \left[-\beta H_{c}\left(U=U_{-}\right)\right]$is the initial thermal state that appears in (5.2). From the criterion (4.19a) one can see that (5.57) correctly describes the local single-particle Green function $G_{i i}^{<}\left(t, t^{\prime}\right)$ in the limit $t, t^{\prime}=\infty, t-t^{\prime}$ fixed: Because $G_{i i}^{<}\left(t, t^{\prime}\right)$ is a singleparticle observable, 4.19a has to be fulfilled only for the trivial case $m=1$, such that the GGE prediction is the same as the diagonal ensemble.

We conclude that the nonthermal stationary state cannot be explained with the fact that the $f$-particle configuration cannot adjust to a new annealed disorder configuration, but it should be related to the fact that the $c$-particle Hamiltonian for a fixed disorder configuration does not lead to thermalization. On the other hand, evaluating any observable in the state (5.54) with Eq. (5.57) is hardly possible. However, for the case of a small quench, $U_{+}=U_{-}+\delta U$, the stationary state Green function can be calculated explicitly in the ensemble (5.57) to first order in $\delta U$. We will do this in the following, and show that the result indeed agrees with the exact DMFT result that follows from $5.30 \mathrm{~b}$ ) and 5.52 . (This can also be taken as an independent check of the exact solution for the quench in the Falicov-Kimball model.)

For a single configuration $\left\{n_{i}^{f}\right\}$ (the dependence on $\left\{n_{i}^{f}\right\}$ is not indicated in the following), the GGE prediction for the local Green function is given by

$$
\begin{aligned}
G_{i i, \mathrm{G}}^{<}\left(t, t^{\prime}\right) & \equiv i \operatorname{Tr}\left[\rho_{\mathrm{G}} \hat{c}_{i}^{\dagger}\left(t^{\prime}\right) \hat{c}_{i}(t)\right] \\
& \stackrel{(i)}{=} i \sum_{\alpha \alpha^{\prime}} e^{i \epsilon_{\alpha^{\prime}} t^{\prime}-i \epsilon_{\alpha} t}\langle i \mid \alpha\rangle\left\langle\alpha^{\prime} \mid i\right\rangle \operatorname{Tr}\left[\rho_{\mathrm{G}} c_{\alpha^{\prime}}^{\dagger} c_{\alpha}\right] \\
& \stackrel{(i i)}{=} i \sum_{\alpha} e^{i \epsilon_{\alpha}\left(t^{\prime}-t\right)}|\langle i \mid \alpha\rangle|^{2} \operatorname{Tr}\left[\rho_{\mathrm{G}} c_{\alpha}^{\dagger} c_{\alpha}\right] \\
& \stackrel{(i i i)}{=} i \sum_{\alpha} e^{i \epsilon_{\alpha}\left(t^{\prime}-t\right)}|\langle i \mid \alpha\rangle|^{2} \operatorname{Tr}\left[\rho_{0} n_{\alpha}\right]
\end{aligned}
$$

In step (i) operators $c_{i}$ are expanded in terms of the $c_{\alpha}$ which have a trivial time evolution $\hat{c}_{\alpha}(t)=e^{-i t \epsilon_{\alpha}} c_{\alpha}$ due to Eq. 5.56, in step (ii) it is used that 5.57) is diagonal in the $n_{\alpha}$, and finally in step (iii) the constraint 5.58) was inserted. The Green function thus depends only on time difference (this is always the case when the Hamiltonian commutes with the density matrix ). We can thus Fourier transform with respect to time difference, 
and average over all sites. This gives

$$
\begin{aligned}
g_{\mathrm{G}}^{<}(\omega) & \equiv \frac{1}{L} \sum_{i} G_{i i, \mathrm{G}}^{<}(\omega) \\
& =\frac{2 \pi i}{L} \sum_{\alpha} \operatorname{Tr}\left[\rho_{0} n_{\alpha}\right] \delta\left(\omega-\epsilon_{\alpha}\right) .
\end{aligned}
$$

For small quenches with $\delta U=U_{+}-U_{-} \rightarrow 0$, the change of $g_{\mathrm{G}}^{<}(\omega)$ with respect to the initial value can be calculated to first order in $\delta U$ from usual perturbation theory, where $\epsilon_{\alpha}$ and $|\alpha\rangle$ are calculated in terms of the eigenvalues $\epsilon_{\alpha-}$ and eigenvectors $|\alpha-\rangle$ for the $c$-particle Hamiltonian $h_{c}$ at $U=U_{-}, \epsilon_{\alpha}=\epsilon_{\alpha-}+\delta U\langle\alpha|\mathcal{F}| \alpha\rangle$ and $\left\langle n_{\alpha}\right\rangle_{0}=\left\langle n_{\alpha-}\right\rangle_{0}+$ $\mathcal{O}\left(\delta U^{2}\right)$. When this is inserted in Eq. 5.64, we get

$$
\left.\frac{\partial}{\partial U_{+}} g_{\mathrm{G}}^{<}(\omega)\right|_{U_{+}=U_{-}}=-w_{1} \frac{\partial}{\partial \omega} r_{\mathrm{G}}^{<}(\omega),
$$

where

$$
r_{\mathrm{G}}^{<}(\omega) \equiv \frac{1}{w_{1} L} \sum_{i} n_{i}^{f} G_{i i}^{<}(\omega)=\frac{2 \pi i}{w_{1} L} \sum_{\alpha} \operatorname{Tr}\left[\rho_{0} n_{\alpha}\right] \delta\left(\omega-\epsilon_{\alpha}\right)\langle\alpha|\mathcal{F}| \alpha\rangle .
$$

is the same as 5.64, but averaged only over site $i$ with $n_{i}^{f}=1$. (Hence $r_{\mathrm{G}}^{<}\left(t-t^{\prime}\right)$ is the site-average of the correlation function $\sqrt[5.12]{ } R_{i i, \mathrm{G}}^{<}=i \operatorname{Tr}\left[f_{i}^{\dagger} f_{i} c_{i}^{\dagger}\left(t^{\prime}\right) c_{i}(t)\right]$.)

In DMFT, on the other hand, results for the long-time limit $\tilde{g}_{\infty}(\omega)=-2 i \operatorname{Im} \tilde{g}_{+}^{\mathrm{r}}(\omega) F(\omega)$ after an infinitesimal quench are obtained from Eq. (5.52) and the derivative of the spectrum (5.25) with respect to $U$. A lengthy calculation gives

$$
\left.\frac{\partial}{\partial U_{+}} \tilde{g}_{+}^{<}(\omega)\right|_{U_{+}=U_{-}}=-w_{1} \frac{\partial}{\partial \omega} \tilde{r}_{+}^{<}(\omega) .
$$

When one assumes that for the homogeneous phase the average (5.63) over all sites (for fixed $\left\{n_{i}^{f}\right\}$ ) can be replaced with the average over all $\left\{n_{i}^{f}\right\}$ one can thus conclude that the exact DMFT result for small quenches is recovered by the stationary state density matrix (5.54) and 5.57).

\subsubsection{Comparison to the strong-coupling expansion}

In this section we compare the exact results for the time-dependent double occupation with the general strong-coupling expansion of Sec. 4.4. It was shown in Sec. 4.4 that after a quench from $U_{-}=0$ to $U=U_{+} \gg V$ the double occupation relaxes to a stationary value $d_{\text {stat }}$ on the fast timescale $(1 / V)$ through damped oscillations with period $2 \pi / U$. The envelope of the damped oscillations is independent of $U$ to first order in $V / U$ [cf. Eq. (4.33)], and $d_{\text {stat }}$ can be evaluated from Eqs. 4.34a and 4.36 for $U_{-}=0$. For the case of half-filling of $c$ - and $f$-particles, $T=0$ in the initial state, and a semielliptic density of states 3.18 this gives

$$
d_{\text {stat }}=\frac{1}{4}-\frac{3}{8 \pi} \frac{V}{U}+\mathcal{O}\left(\frac{V^{2}}{U^{2}}\right)
$$




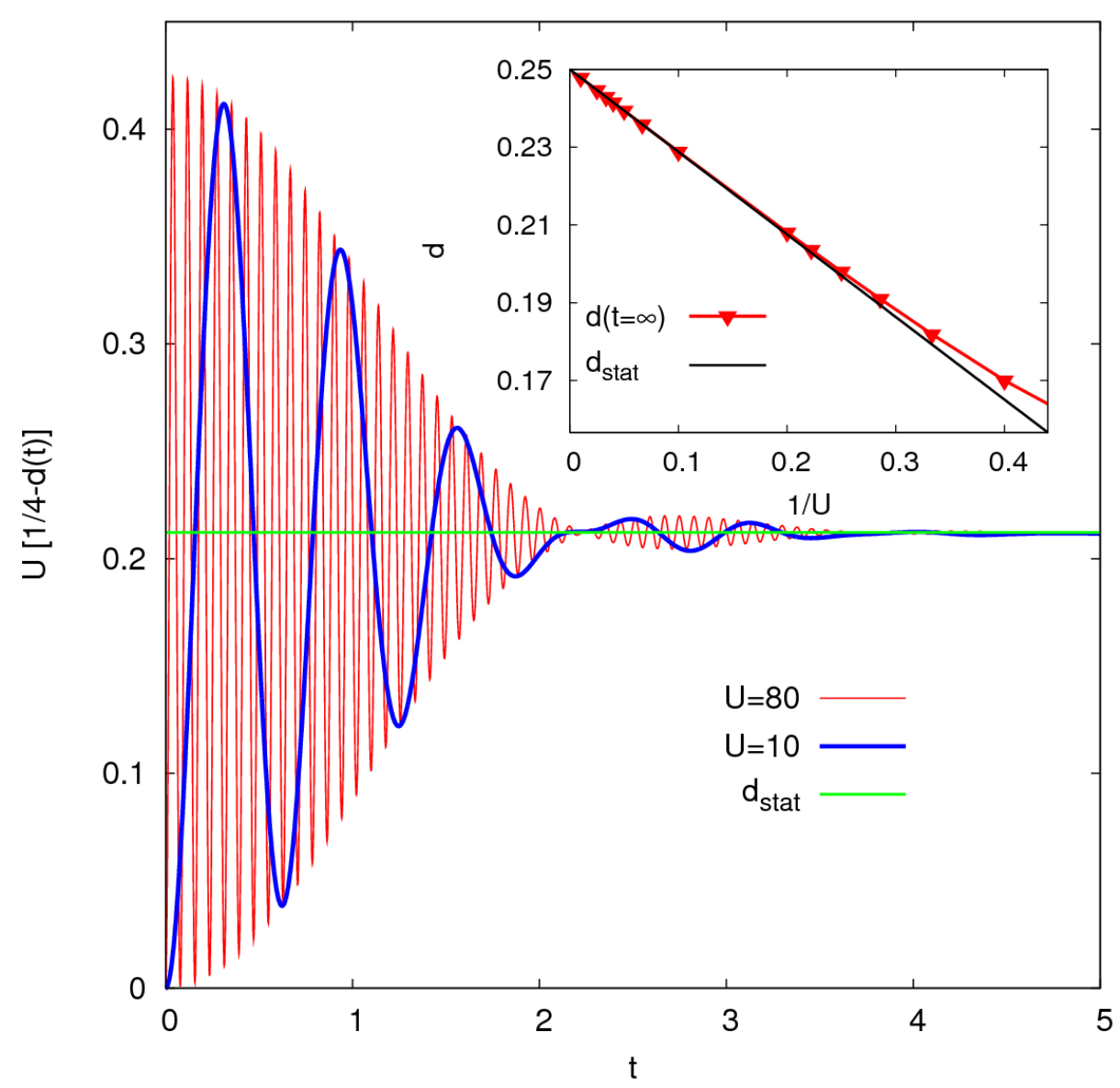

Figure 5.6: Difference of the time-dependent double occupation $d(t)$ to the initial value $d=1 / 4$ for quenches from $U_{-}=0(T=0)$ to $U_{+}=10$ and 80 . The horizontal line corresponds to the quasi-stationary value (5.68) to which $d(t)$ is predicted to relax in the strong-coupling expansion. Inset: The exact $t=\infty$ limit (5.49) (triangle symbols), compared to the stationary value (5.68) of the strong-coupling expansion for $U \rightarrow \infty$. 
Here we used in Eq. 4.36 that only one spin-species is immobile, i.e., $\rho_{\uparrow}(\epsilon)=0$ and $\rho_{\downarrow}(\epsilon)$ $=\sqrt{4 V^{2}-\epsilon^{2}} / 2 \pi V$ in the expectation value $E_{k i n, 0}^{\downarrow}$ [Eq. 33.33 ]. In the left panel of Fig 5.6 the correction of $d(t)$ to the initial value $d=1 / 4$ is plotted. This agrees very well with the strong coupling prediction. The envelope of the oscillations is indeed almost independent of $U$, and $d(t)$ quickly decays to the value (5.68).

For the Falicov-Kimball model one can go one step further and show that the stationary value (5.68) from strong-coupling perturbation theory agrees with the exact long-time limit (5.49) to first order in $V / U$ (Fig. 5.6, inset). It was mentioned in Sec. 4.5 that a unitary expansion around an integrable point in general, and the strong-coupling expansion of the Hubbard model in particular (Sec. 4.4) imply nonthermal behavior, but that the series need not converge and thermalization may thus occur on exponentially long timescales. In contrast, for the Falicov-Kimball model such exponentially slow dynamics is absent, and the strong coupling expansion for $d$ indeed converges to the exact nonthermal stationary value for the Falicov-Kimball model. This finding is quite remarkable because the nonthermal behavior in the Falicov-Kimball model is related to the existence of infinitely many conserved quantities, an explanation which is quite distict from the validity of the strong coupling expansion. Analogous results hold for the $1 / r$ Hubbard chain [126], where the long-time limit can be evaluated exactly and coincides with the value obtained from Eqs. (4.34) and (4.35), $\Delta d=(V / U)(1-2 n / 3) \pi[126$. 


\section{Chapter 6}

\section{Interaction quench in the Hubbard model}

\subsection{Preliminary remarks}

The dynamics of the Falicov-Kimball model (previous chapter) resembles that of a constrained integrable system. Because this behavior was traced back to the immobility of one spin species, one can expect completely different results for the nonintegrable Hubbard model. The relaxation of the Hubbard model after a quench was already discussed in Sec. 4.4 for both weak coupling $(U \ll V)$ and and strong coupling $(V \ll U)$, where the system is trapped away from thermal equilibrium on long timescales $V / U^{2}$ and $U / V^{2}$, respectively. The intermediate coupling regime is only accessible by numerical methods. It has been studied for the one-dimensional bosonic Hubbard model [124] using timedependent DMRG. While DMRG works best for one-dimensional systems, dimensions $d>1$ are particulary interesting for the fermionic Hubbard model, because the latter is integrable via Bethe ansatz in $d=1$ [4]. In this chapter we investigate this model in the opposite limit of infinite dimensions, using nonequilibrium DMFT, and investigate the relaxation after quenches from the noininteracting state to a wide range of interaction parameters $U$ [74].

The following chapter repeats the analysis of Ch. 5 for the Hubbard model (1.1). Only the paramagnetic phase at half-filling is considered. The system is initially in the ground state of the noninteracting Hamiltonian, and at $t=0$ the Coulomb repulsion is switched to a finite value, $U(t \geq 0)=U$. The time-dependent Hamiltonian is thus given by

$$
H(t)=\sum_{i j \sigma} V_{i j} c_{i \sigma}^{\dagger} c_{j \sigma}+\Theta(t) U \sum_{i}\left(n_{i \uparrow}-\frac{1}{2}\right)\left(n_{i \downarrow}-\frac{1}{2}\right) .
$$

Hoppings $V_{i j} \equiv V t_{i j}$ are chosen corresponding to a semi-elliptic density of states [Eq. (3.18)], energy is measured in units of the quarter-bandwidth $V$ and time in units of $1 / V$. The self-consistency takes the closed form (3.19) due to the semielliptic density of states. The DMFT single-site problem is solved by means of the weak-coupling auxiliary-field continuous-time Monte Carlo (CTQMC) algorithm (Sec. 3.4.2). This method is highly suitable for noninteracting initial states, because the imaginary branch of the contour 


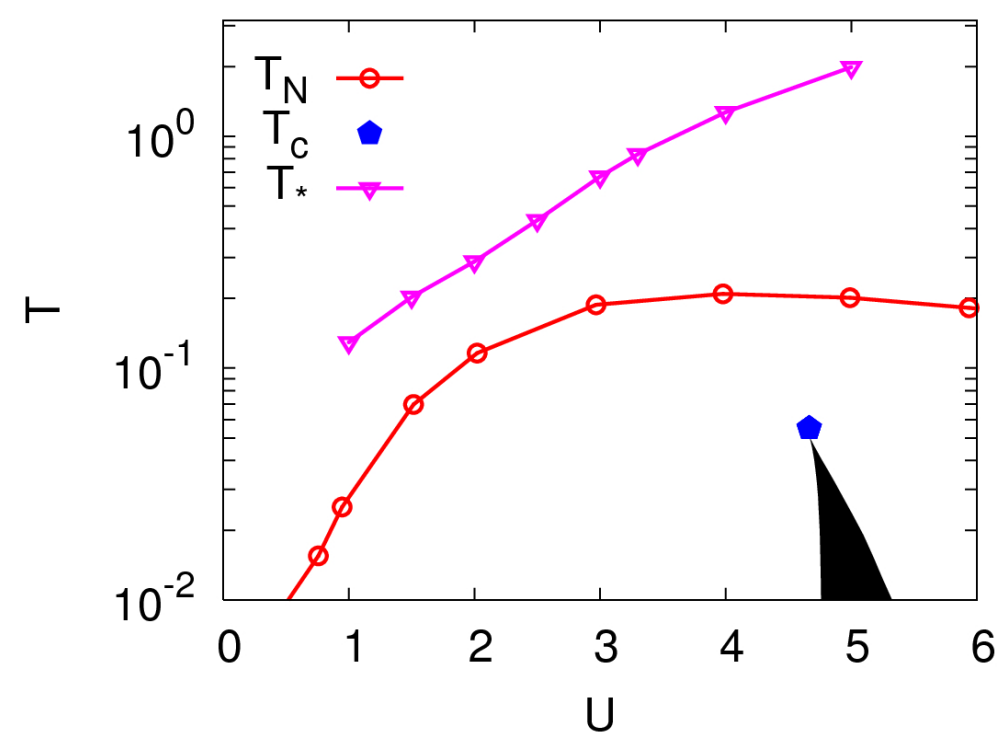

Figure 6.1: Effective temperature $T_{*}$ after an interaction quench in the Hubbard model, compared to the Neel temperature $T_{N}$ [179], and the first-order Mott transition in the homogeneous phase. The filled area marks the coexistence regime between metallic and insulating phase in equilibrium, and the blue diamond symbol denotes the critical endpoint of the first-order transition $\left(U_{c} \approx 4.67 \mathrm{~V}, T_{c} \approx 0.055 \mathrm{~V}\right.$, taken from Ref. [180]).

does not enter the CTQMC calculation. Imaginary times can be transformed to real frequencies (Sec. 2.2.3), and thus we can treat also initial states at zero temperature. After convergence is reached, observables of the lattice system are calculated from the local Green function $G_{\sigma}\left(t, t^{\prime}\right)$ and the self-energy $\Sigma_{\sigma}\left(t, t^{\prime}\right)$ (cf. Sec. 3.3). In the following, the double occupation $d(t)=\left\langle\hat{n}_{i \uparrow}(t) \hat{n}_{i \downarrow}(t)\right\rangle$ [Eq. (3.39]), the momentum distribution $n\left(\epsilon_{\boldsymbol{k}}, t\right)$ $=\left\langle\hat{c}_{\boldsymbol{k} \sigma}^{\dagger}(t) \hat{c}_{\boldsymbol{k} \sigma}(t)\right\rangle$ [Eq. 3.31, and the kinetic energy per lattice site, $E_{k i n}(t)$ [Eq. 3.32b] are considered. Further numerical details are deferred to App. A.

\section{The effective temperature}

To test for thermalization, expectation values of various observables in the nonequilibrium state are compared to thermal expectation values. The corresponding effective grandcanonical ensemble $\rho_{*}=\exp \left[-\left(H-\mu_{*} N\right) / T_{*}\right] / Z_{*}$ is determined by fixing $T_{*}$ and $\mu_{*}$ such that $E_{t h} \equiv \operatorname{Tr}\left[\rho_{*} H\right]=E$ and $N_{t h} \equiv \operatorname{Tr}\left[\rho_{*} N\right]=N$, where $H$ is Hamiltonian (6.1) after the quench, $N$ is the total particle number, and $E=\langle\hat{H}(t>0)\rangle_{0}$ is the (constant) energy after the quench [cf. Eq. (4.8)]. (For half-filling, we have $\mu_{*}=0$.) All equilibrium expectation values are calculated using equilibrium DMFT, with Hirsch-Fye QMC as impurity solver (using the existing Augsburg implementation with Blümer smoothing, see Ref. [180]). Already for relatively small $U(U \geq 1)$ the excitation of the system after the quench is so large that $T_{*}$ lies well above the first-order Mott transition in the paramagnetic phase and the Neel-temperature $T_{N}$ of the antiferromagnetic phase at half-filling (Fig. 

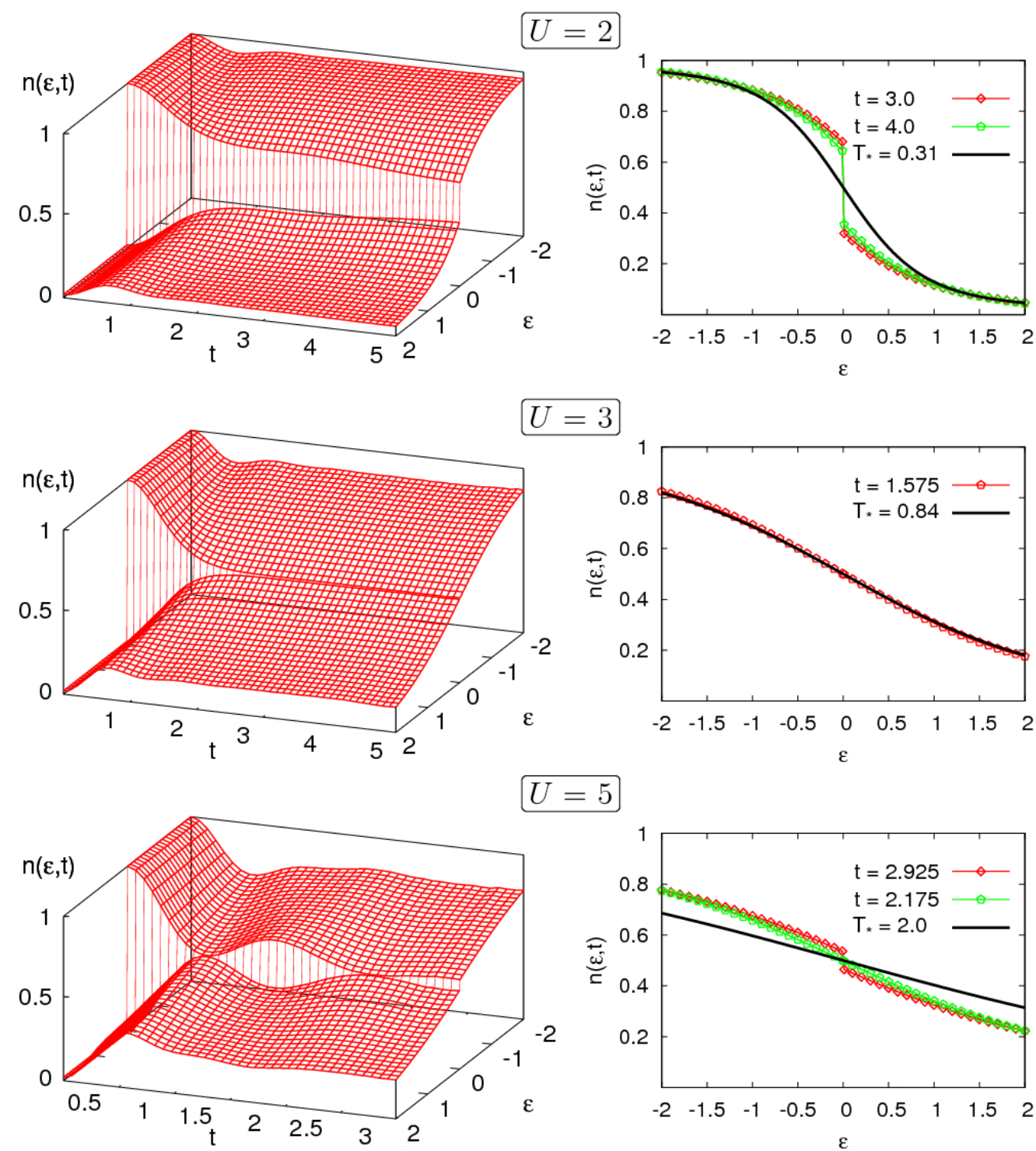

Figure 6.2: Right column: Momentum distribution $n\left(\epsilon_{\boldsymbol{k}}, t\right)$ for quenches from $U=0$ to $U=2, U=3$, and $U=5$ (from top to bottom). Left column: $n\left(\epsilon_{\boldsymbol{k}}, t\right)$ at given times (symbols), compared to the momentum distribution in a thermal state the effective temperature $T_{*}$ (solid lines). For $U=5$ (bottom), times are chosen at the last accessible collapse minimum $(t \approx 2.175)$ and revival maximum $t \approx 2.925$. 

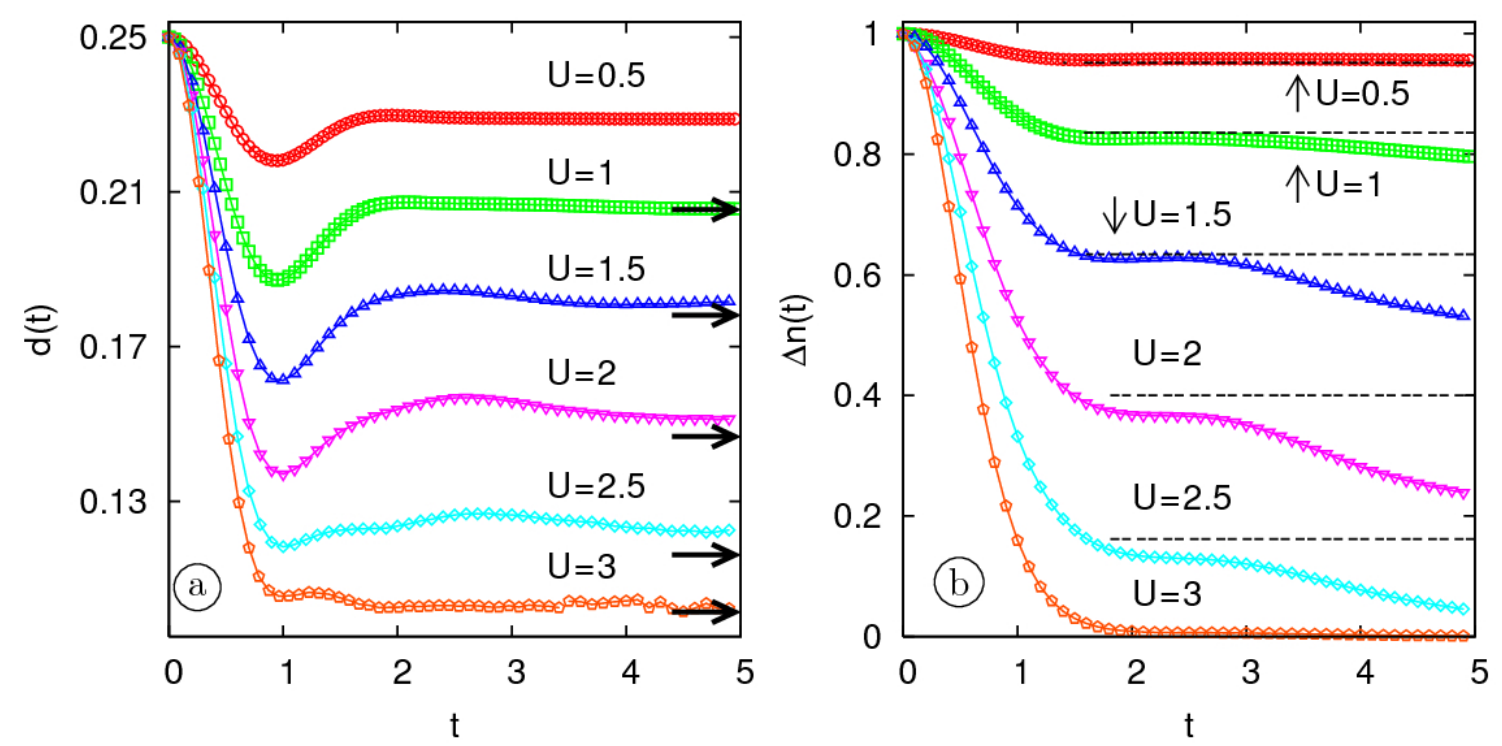

Figure 6.3: (Reprinted from Ref. [74]) Fermi surface discontinuity $\Delta n$ (b) and double occupation $d(t)$ (a) after quenches to $U \leq 3$. Horizontal dotted lines in (a) are at the quasistationary value $\Delta n_{\text {stat }}=2 Z-1$ predicted in Ref. [127], with the $T=0$ quasiparticle weight $Z$ taken from equilibrium DMFT data [82. Horizontal arrows in (b) indicate corresponding thermal values $d_{\text {th }}$ of the double occupation, obtained from equilibrium DMFT using QMC.

6.1). Nevertheless, the physics of the Hubbard model is not trivial at such high energies, as we will see below. On the contrary, even in the equilibrium phase diagram it is not understood in simple terms why the critical temperature of the Mott transition (blue diamond symbol in Fig. 6.1) is two orders of magnitude smaller than the bare energy scales $V$ and $U$.

\subsection{Collapse of the Fermi-surface discontinuity}

Fig. 6.2 shows the momentum distribution $n(\epsilon, t)$ after a quench, which evolves from a step function in the initial state to a continuous function of $\epsilon$ at large times. In equilibrium, a true discontinuity in the momentum distribution at the Fermi energy $\epsilon=0$, whose size is then given by the quasiparticle weight $Z$, exists only in the Fermi-liquid state at temperature $T=0$. Since the Hubbard model system is excited by the quench, one would thus expect that the discontinuity disappears in the final state. In fact, in the numerical data we observe that the the discontinuity remains sharp for $t>0$, and only its height decays smoothly. Pictorially, the Fermi sea does not melt from the edge, but reorganization of the distribution happens simultaneously at all momenta.1

This robustness of the Fermi surface can be proven directly from the lattice Dyson

\footnotetext{
${ }^{1}$ An instantaneous collapse of the discontinuity would require that $\partial_{t} n(\epsilon, t)$ diverges where $\partial_{\epsilon} n(\epsilon, t)$ diverges, as for a hypothetical diffusion process in $\boldsymbol{k}$-space.
} 
equation (3.14), using only the local nature of the the local self-energy $\Sigma\left(t, t^{\prime}\right)$. For a noninteracting initial state at half-filling, the discontinuity $\Delta n(t)=n\left(0^{-}, t\right)-n\left(0^{+}, t\right)$ can be expressed as

$$
\Delta n(t)=\left|G_{\epsilon=0, \sigma}^{\mathrm{r}}(t, 0)\right|^{2},
$$

in terms of the retarded Green function $G_{\epsilon_{k} \sigma}^{\mathrm{r}}(t, 0)$. The operator representation $G_{\epsilon_{k} \sigma}^{\mathrm{r}}(t, 0)$ $=-i \Theta(t)\left\langle\left\{\hat{c}_{\boldsymbol{k} \sigma}(0), \hat{c}_{\boldsymbol{k} \sigma}^{\dagger}(t)\right\}\right\rangle$ of this function (cf. Table 2.2 clearly shows that the collapse of the discontinuity $\Delta n$ is closely related to the decay of electron and hole excitations which are created at time $t=0$ at the Fermi surface.

In the following we prove Eq. (6.2) from Eqs. (3.31) and (3.14) under the assumption of a $\boldsymbol{k}$-independent self-energy. The following argument is quite technical and is not necessary for the understanding of the discussion in the following sections. Eq. (3.14) has the form (2.36), with the replacement $\Lambda=\Sigma_{\sigma}, G=G_{\epsilon \sigma}$, and $h(t)=\epsilon$. Thus $G_{\epsilon}^{\mathrm{r}}$ is determined by the equation

$$
\left(i \partial_{t}-\epsilon\right) G_{\epsilon \sigma}^{\mathrm{r}}\left(t, t^{\prime}\right)-\int_{t^{\prime}}^{t} d \bar{t} \Sigma_{\sigma}^{\mathrm{r}}(t, \bar{t}) G_{\epsilon \sigma}^{\mathrm{r}}\left(\bar{t}, t^{\prime}\right)=0
$$

for $t>t^{\prime}$, with initial condition $G_{\epsilon \sigma}^{\mathrm{r}}(t, t)=-i$ [cf. Eq. (2.38)]. Because the self-energy is independent of $\epsilon$, the retarded function is a continuous function of $\epsilon$ at $\epsilon=0$. The lesser component follows from Eq. 2.42, with the replacements intriduced above,

$$
\left(i \partial_{t}-\epsilon\right) G_{\epsilon \sigma}^{<}\left(t, t^{\prime}\right)-\int_{0}^{t} d \bar{t} \Sigma^{\mathrm{r}}(t, \bar{t}) G_{\epsilon \sigma}^{<}\left(\bar{t}, t^{\prime}\right)=\int_{0}^{t^{\prime}} d \bar{t} \Sigma^{<}(t, \bar{t}) G_{\epsilon \sigma}^{\mathrm{a}}\left(\bar{t}, t^{\prime}\right) .
$$

Here we have used that the self-energy vanishes in the initial state $\left(\Sigma^{\mathrm{M}}=\Sigma\ulcorner=0)\right.$. Next we define the contour function $F_{\sigma}=G_{\epsilon=0^{-} \sigma}-G_{\epsilon=0^{+} \sigma}$, which satisfies

$$
-i F_{\sigma}^{<}(t, t)=\Delta n(t)
$$

due to Eq. (3.31). Taking the the difference of Eq. 6.4 at $\epsilon=0^{-}$and $\epsilon=0^{+}$yields

$$
i \partial_{t} F_{\sigma}^{<}\left(t, t^{\prime \prime}\right)-\int_{0}^{t} d \bar{t} \Sigma^{\mathrm{r}}(t, \bar{t}) F_{\sigma}^{<}\left(\bar{t}, t^{\prime \prime}\right)=0,
$$

where we have used that $G_{\epsilon \sigma}^{\mathrm{a}}$ is continuous at $\epsilon=0$. Eq. 6.6 is the same as the retarded equation 6.3) for $\epsilon=0$ and $t^{\prime}=0$. Hence the solution is given by

$$
F_{\sigma}^{<}\left(t, t^{\prime \prime}\right)=i F_{\sigma}^{<}\left(0, t^{\prime \prime}\right) G_{\epsilon=0, \sigma}^{\mathrm{r}}(t, 0)
$$

where the factor $i F_{\sigma}^{<}\left(0, t^{\prime \prime}\right)$ accounts for the different initial conditions in Eqs. (6.3) and (6.6). It remains to evaluate the initial value $F_{\sigma}^{<}(0, t)$, which is given by $F_{\sigma}^{<}(0, t)=$ $-F_{\sigma}^{\ulcorner}(\beta, t)$ [cf. Eq. 2.42b]]. The mixed components $G_{\epsilon \sigma}^{\ulcorner}(\tau, t)$ are obtained from Eq. 2.41, with the same replacements as above. Using again that mixed and Matsubara components of $\Sigma$ vanish, we obtain

$$
\left(-\partial_{\tau}-\epsilon\right) G_{\epsilon \sigma}^{\ulcorner}(\tau, t)=0
$$

which must be solved with the boundary condition $G_{\epsilon \sigma}^{\ulcorner}(\beta, t)=-G_{\epsilon \sigma}^{\ulcorner}(0, t)-G_{\epsilon \sigma}^{\mathrm{a}}(0, t)[\mathrm{cf}$. Eq 2.41b]. A straigtforward calculation thus yields $G_{\epsilon \sigma}^{\leftarrow}(\beta, t)=-\Theta(-\epsilon) G_{\epsilon \sigma}^{\mathrm{a}}(0, t)$, and thus $F_{\sigma}^{<}(0, t)=-F_{\sigma}^{\ulcorner}(\beta, t)=G_{\epsilon=0 \sigma}^{\mathrm{a}}(0, t)$. Together with Eqs. (6.5) and 6.7) and the symmetry 2.15a this proves the proposition 6.2 . 
Figure 6.4: (Reprinted from Ref. [74]) Fermi surface discontinuity $\Delta n$ (Left panel) and double occupation $d(t)$ (right panel) after quenches to $U \geq 3.5$. Horizontal arrows indicate corresponding thermal values $d_{\mathrm{th}}$ of the double occupation, obtained from equilibrium DMFT using QMC. Inset: thermal value $d_{\mathrm{th}}$ and $d_{\text {med }}$, the average of the first maximum and the second minimum of $d(t)$, which provides an estimate of the stationary value $d_{\text {stat }}[$ Eq. (6.9)]. Black dotted lines are the respective results from the strong-coupling expansion (see text).

\subsection{Relaxation after the quench}

In the following section the relaxation after the quench is characterized by means of the distribution $n(\epsilon, t)$ and its discontinuity Eq. (6.2), and the double occupation $d(t)$. As already apparent from Fig. 6.2, the relaxation is qualitatively different in the weakcoupling and strong-coupling regimes and at intermediate coupling. These three regimes are now discussed separately.

\section{Weak-coupling regime}

The double occupation $d(t)$ and the Fermi surface discontinuity $\Delta n(t)$ are plotted for quenches to $U \leq 3 \mathrm{~V}$ in Fig. 6.3. The double occupation $d(t)$ relaxes from its initial uncorrelated value $d(0)=\left\langle n_{\uparrow}\right\rangle_{0}\left\langle n_{\downarrow}\right\rangle_{0}=1 / 4$ almost to its thermal value $d_{\mathrm{th}}$, while $\Delta n(t)$ remains finite for times $t \leq 5 / \mathrm{V}$. Relaxation of $\Delta n(t)$ apparently proceeds in two steps: A fast drop to a plateau on a timescale that is almost independent of $U$ is followed by a slower decrease of $\Delta n(t)$ towards $\Delta n=0$. For small $U(U=0.5$ in Fig. 6.3), $\Delta n(t)$ even approaches a positive value which remains constant within the accessible times. These findings are in agreement with the prethermalization after interaction quenches in the Hubbard model at weak coupling that has been found previously [127]: Using the the perturbative flow equation analysis for $U \ll V$ Moeckel and Kehrein were able to prove that after an interaction quench in the Hubbard model a quasistationary state is formed on time scales on the order of $V / U^{2}$, in which the double occupancy is given by $d_{\text {stat }}=$ $d_{\text {th }}+\mathcal{O}\left(U^{3} / V^{3}\right)$ and the momentum distribution retains a finite discontinuity $\Delta n_{\text {stat }}$ at the Fermi surface. The latter is given by $\Delta n_{\text {stat }}=2 Z-1$, where $Z$ is the quasiparticle weight in the ground state at interaction $U$. Furthermore, it was argued in Ref. [127] that full thermalization could be obtained within the next order of the flow equation approach, and would thus occur on the much longer timescales $V^{3} / U^{4}$ (Sec. 4.4).

Our numerical data agree very well with the predicted value of $\Delta n_{\text {stat }}$ for $U \leq 1 \mathrm{~V}$ around $t=2 / V$. Even for quenches to larger $U$, a prethermalization plateau remains visible in Fig. 6.3a at roughly this value, although there are already sizable deviations from the weak-coupling prethermalization discussed in Ref. [127] at these large values of $U$. On the one hand, prethermalization is less pronounced because the timescales $V / U^{2}$ and $V^{3} / U^{4}$ are no longer well separated, and the decay of $\Delta n$ after the initial drop is not much slower than the initial decay. On the other hand, the nonthermal nature of the intermediate state becomes also observable in the double occupation, which clearly 
deviates from the thermal value at $t=5$ and $1.5 \leq U \leq 2.5$. In the weak-coupling expansion [127, potential and kinetic energy have already reached their final values after prethermalization.

\section{Strong-coupling regime}

For quenches to large $U$ collapse-and-revival oscillations with approximate frequency $2 \pi / U$ occur both in $d(t)$ and $\Delta n(t)$ (Fig. 6.4). This phenomenon is well understood for the strong coupling limit of the Hubbard model (Sec. 4.4) and has been discussed in detail for the Falicov-Kimball model (Sec. 5.3.4). As expected from the strong-coupling expansion in Sec. 4.4, the oscillations are damped for nonzero $V$ on time scales on the order of $1 / V$. Interestingly, the first few oscillations of $d(t)$ are not centered around the thermal value $d_{\mathrm{th}}$ (solid arrows in Fig. 6.4 b), but the latter is instead located close to the first minimum of $d(t)$. These numerical results are also in good quantitative agreement with the asymptotic results of Sec. 4.4 (Inset in Fig. 6.4): Analogous to the Falicov-Kimball model [Eq. 5.68 we obtain from Eqs. (4.34), 4.36) and (3.33) for the Hubbard model (at half-filling, $T=$ 0 , and a semielliptic density of states)

$$
d_{\text {stat }}=\frac{1}{4}-\frac{3}{4 \pi} \frac{V}{U}+\mathcal{O}\left(\frac{V^{2}}{U^{2}}\right)
$$

while the thermal value is given by $d_{t h}=\left[d(0)-d_{\mathrm{th}}\right] / 2$ [Eq. 44.37)]. Both $d_{\text {stat }}$ and $d_{t h}$ agree well with the numerical DMFT results (inset in Fig. 6.4). Deviations to the thermal state can also be seen in the momentum occupation. For example, at $U=5$ the momentum occupation $n(\epsilon, t)$ oscillates around a distribution that is steeper than the thermal one, and therefore closer to the initial distribution (Fig. 6.2, bottom right). Interestingly, the differences between $n(\epsilon, t)$ and the thermal distribution are most pronounced away from the Fermi surface, while for the weak-coupling case they are most prominent at the Fermi surface (Fig. 6.2, top right).

\section{Rapid thermalization, $U \approx U_{c}^{\text {dyn }}=3.2 \mathrm{~V}$}

The numerical results that were presented so far confirm the prethermalization around the noninteracting case and the atomic limit. Interestingly, the signatures of prethermalization prevail quite far from the two respective limits (Fig. 6.5). As apparent from the Fermi surface discontinuity $\Delta n_{1}$ at its first revival maximum (Fig. 6.5 a), the collapse-and-revival oscillations of the strong-coupling regime disappear for quenches to $U$ between $3.3 \mathrm{~V}$ and $3 V$. Conversely, the weak-coupling prethermalization plateau $\Delta n_{\text {stat }}$, which we define by the deflection point in $\Delta n(t)$, exists only for $U \lesssim 3.3 \mathrm{~V}$. At $U \approx 3.3 \mathrm{~V}$, the system relaxes rapidly to thermal equilibrium, siuch that momentum distribution (Fig.6.2b) and double occupation (Fig 6.4) reach their respective thermal values already before the first expected collapse-and-revival oscillation at time $2 \pi / U$. Within the numerical accuracy no deviations between the thermal values and the relaxed values can be seen 2 The sta-

\footnotetext{
2 To estimate the numerical error in $d(t)$, which is due to both statistical Monte Carlo errors and discretization errors that arrise from the solution of contour equations, we calculate the deviation $\Delta E(t)$
} 


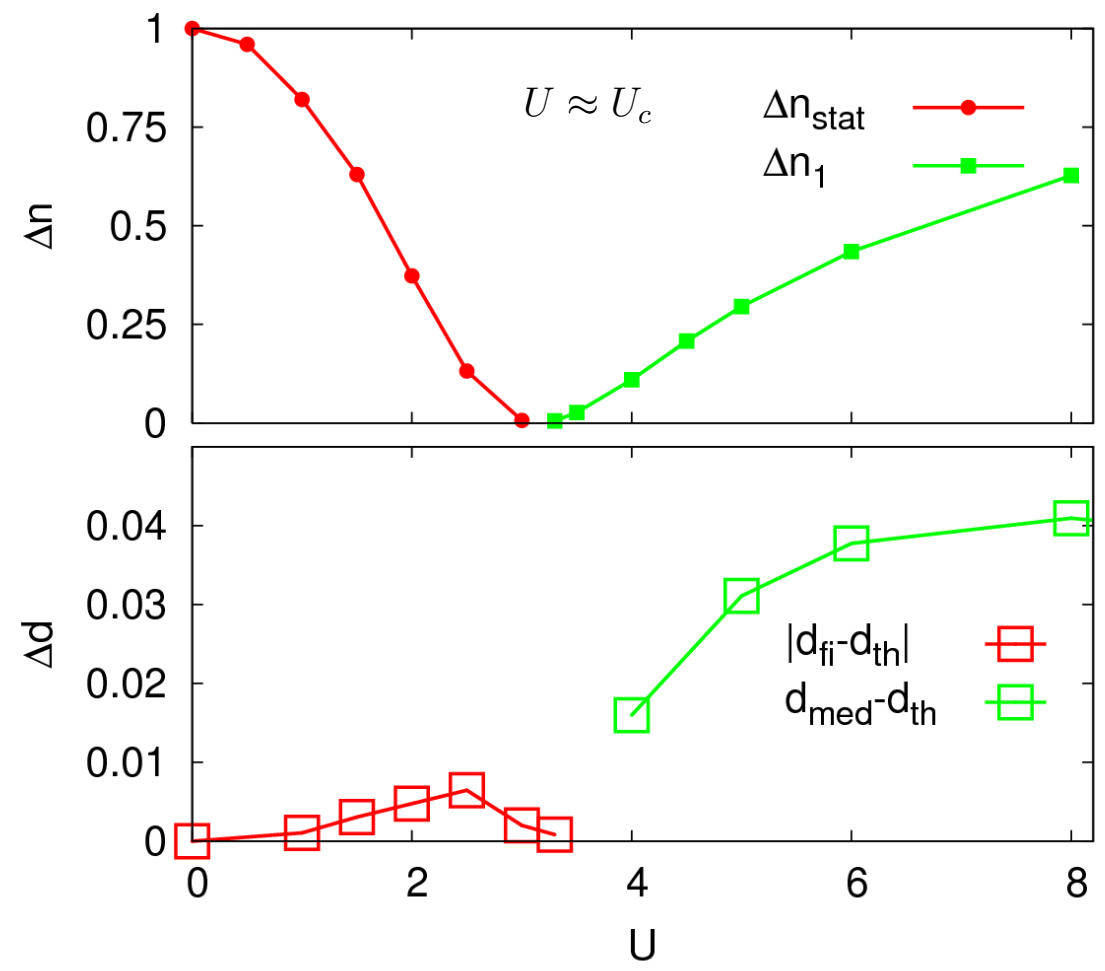

Figure 6.5: Upper panel: $U$-dependence of the prethermalization plateau, $\Delta n_{\text {stat }}$, and the first revival maximum, $\Delta n_{1}$ of the jump in the momentum distribution. Lower panel: Difference of the double occupation $d_{f i}(d(t)$, averaged for $4 \leq t \leq 5)$ to the thermal value $d_{t h}$ (dots). Difference of the double occupation $d_{\text {med }}$ (cf. inset in Fig. 6.5) and $d_{t h}$. The shaded area marks the region near $U_{c}^{\text {dyn }}=3.2 \mathrm{~V}$ with very fast thermalization.

tionary state can also be characterized by its spectral function, which develops the three peak structure of the correlated metal on a similar short time scale (Sec. 8.4.1).

This sensitive dependence of the relaxation on the interaction suggests that the prethermalization regimes at weak and strong Hubbard interaction are separated by a special point, which we estimate from our data to be located at $U_{c}^{\text {dyn }}=3.2 V$. This finding is quite remarkable in view of the fact that the effective temperature $T_{*}$ after the quench is much higher than the critical endpoint of the Mott metal-insulator transition in equilibrium (Fig. 6.1. $T_{*}=0.84 \mathrm{~V}$ for $U=3.3 \mathrm{~V}$ ), so that in equilibrium metallic and insulating phases could hardly be distinguished. A similar critical behavior was found for quenches in Heisenberg chains [135].

This behavior may indicate the existence of a dynamical phase transition, at which relaxation processes on all energy scales become relevant. However, the notion of a dynamical phase transition itself needs to be clarified in more detail. Our results show a qualitative change in the short-time relaxation behavior, which coincides with a change in

of the measured energy $E(t)$ from the conserved value $E(t=0)$. The maximum of $|\Delta E| / L U$ is on the order of the symbol size in Figs. 6.3, 6.4, and 6.5. 
the long-time behavior from slow thermalization to rapid thermalization, (if thermalization occurs at all for $U>U_{c}^{\text {dyn }}$ and $U<U_{c}^{\text {dyn }}$ ). The change in the short-time dynamics reflects a change in the nature of single-particle excitations [Eq. 6.2] ]. It occurs also in equilibrium even at very high temperatures, because $\left|G_{\epsilon \sigma}^{\mathrm{r}}\left(t-t^{\prime}\right)\right|^{2}$ becomes oscillatory upon the transfer of spectral weight to the Hubbard subbands at $\pm U$. On the other hand, the change in the long-time behavior cannot be further quantified within the current analysis. Thermalization may occur on different timescales $U>U_{c}^{\text {dyn }}$ and $U<U_{c}^{\text {dyn }}$, but our results are also consistent with a transition from nonergodic to ergodic behavior depending on excitation energy or deviation form integrability (Sec. 4.5). The next important step towards further understanding of the dynamical transition would be to repeat the present analysis for different initial states, and thus test whether the transition is universal in the sense that some of its properties depend only on the excitation energy and the interaction $U$. This requires only few modifications in the current numerical algorithm and should be possible in the near future.

In spite of these open questions, the results of this chapter form a prediction for experiment that may be checked in the near future, using cold atomic gases. Unfortunately, the measurement of the momentum distribution is quite subtle. First of all, the latter is not a local quantity and thus more sensitive to the trapping potential. Furthermore, its measurement requires an adiabatic ramp-down of the lattice to map quasi-momenta to real momenta, which are then recorded by time-of flight imaging [181]. Although one could freeze the dynamics of the momentum occupation by switching off the interaction before this ramp down, this additional experimental will cause further expoerimental difficulties. On the other hand, the momentum occupation is directly accessible. We therefore conclude that breakdown of the prethermalization regime at strong coupling below some finite critical interaction is the signature that one should look for first in experiment [Fig. 6.5, lower panel]. 


\section{Chapter 7}

\section{Slow changes of the Hamiltonian: The crossover to adiabatic behavior}

\subsection{Introduction}

The term adiabatic is used in thermodynamics for quasistatic processes without heat exchange with the environment. The entropy remains constant during an adiabatic process, while it always increases when the process takes place in a finite time, i.e., when it is no longer quasistatic. These fundamental concepts are closely related to the adiabatic theorem in quantum mechanics, which states that a quantum system that is initially in the ground state remains in the ground state during an infinitesimally slow modification of the Hamiltonian, while it is excited when the Hamiltonian changes more rapidly in time. The paradigm for this crossover from adiabatic to non-adiabatic behavior in quantum system is the exactly solvable Landau-Zener model [182, 183], i.e., a two-level system $H_{L Z}(t)=$ $v t \sigma_{z}+\gamma \sigma_{x}$ that is driven through an avoided level crossing with finite speed $v>0\left(\sigma_{z}\right.$ and $\sigma_{x}$ are Pauli matrices). When the system is in the ground state $\left|\phi_{0}(-\infty)\right\rangle=(1,0)^{+}$ at time $t=-\infty$, the probability to find the system in the excited state $\left|\phi_{1}(\infty)\right\rangle=(1,0)^{+}$ at $t \rightarrow \infty$ is exponentially small when the speed $v$ is small compared to the scale $\gamma^{2}$ set by the gap $\gamma$ at the avoided crossing, $\left.\left|\left\langle\psi(t \rightarrow \infty)|| \phi_{1}(\infty)\right\rangle\right\rangle\right|^{2} \sim \exp \left(-\gamma^{2} / \pi v\right)$.

In many-body systems, a change of the Hamiltonian usually involves many level crossings which cannot be treated independently, and few analytic results exist in the literature [184]. In the following we consider a ramp, in which the system is initially in the ground state and some parameter of the Hamiltonian is changed between two fixed values within a time $\tau_{d}$, and investigate the crossover from the extreme non-adiabatic limit $\tau_{d}=0$ (the quench) to possibly adiabatic behavior in the limit $\tau_{d} \rightarrow \infty$. A measure for the degree of non-adiabaticity in such a ramp is the excitation energy $\Delta E\left(\tau_{d}\right)$ after the ramp with respect to the new ground state 1

1 For initial states at finite temperature, the increase of entropy would a better measure of nonadiabaticity. However, entropy is only uniquely defined for thermal equilibrium states, and thus it can only be computed after the ramp is finished, assuming that the system thermalizes. On the other hand, thermalization is not possible in some models, e.g., the Falicov-Kimball model (Ch. 5). One cannot expect that a system which does not thermalize after quench stays in thermal equilibrium during a ramp, even 
The system is considered to behave adiabatic when $\Delta E\left(\tau_{d}\right)$ vanishes for $\tau_{d} \rightarrow \infty$. It is expected that the excitation energy is still small for $\tau_{d}<\infty$, just as in the LandauZener formula, when the ground state is protected by a gap for all parameters throughout the ramp [185]. However, it is not exponentially small in general: As we will see below, $\Delta E\left(\tau_{d}\right)$ strongly depends on the ramp shape when the system is gapped. It can be made exponentially small $\Delta E\left(\tau_{d}\right) \propto \exp \left(\right.$ const. $\left./ \tau_{d}\right)$ for certain ramps, but often $\Delta E\left(\tau_{d}\right)$ vanishes only algebraically. This is in principle already known from the Landau-Zener model, where the excitation is exponentially small only when the avoided level crossing is traversed from $t=-\infty$ to $t=+\infty$, whereas the excitation probability is proportional to $1 / \tau_{d}^{2}$ if the evolution is started exactly at the center of the level crossing [186].

The situation is completely different for gapless systems. In this context ramps across quantum critical points have attracted some attention recently. For example, in the exactly solvable one-dimensional transverse-field Ising model the gap vanishes at exactly one value of the transverse field, namely the critical point. When the magnetic field is ramped across this point one finds [187, 188, 189]

$$
\Delta E\left(\tau_{d}\right) \sim \tau_{d}^{-\eta}
$$

for $\tau_{d} \rightarrow \infty$, with a rational exponent $\eta=\frac{1}{2}$. Similar results have since then been obtained for a number of other quantum critical systems, such as the Bose-Hubbard model [190] or the random field Ising model [191]. However, the existence of a quantum critical point is not necessary to obtain a non-analytic relation $\Delta E\left(\tau_{d}\right)$ [185, 192]. Eq. (7.1), with various values of the exponent $\eta$, holds for ramps within gapless phases in several gapless systems [185]. For a continuous bath of harmonic oscillators, which model the low-energy excitations of many systems, the exponent $\eta$ for a slow squeeze of the oscillator mass depends on the spatial dimension (the bath density of states) [185]: An analytic relation $\Delta E\left(\tau_{d}\right) \sim \tau_{d}^{-2}$ is found for all dimensions $d \geq 3$, while $\eta$ is non-integer for $d=2$. For $d=1$, the thermodynamic limit does not commute with the limit of large $\tau_{d}$, i.e., the prefactor in Eq. (7.1) increases with system size [185].

In this Chapter we calculate $\Delta E\left(\tau_{d}\right)$ for ramps in the Falicov-Kimball model, using nonequilibrium DMFT. We find that Eq. (7.1) holds for $\tau_{d} \rightarrow \infty$. The exponent differs for ramps over the metal-insulator transition, within the metallic phase, and within the insulating phase. For the latter two cases, this behavior can be explained by a simple perturbative argument.

The excitation energy during a non-adiabatic ramp, and its dependence on $\tau_{d}$ is not only a fundamental property of the many-body system, but it is also of practical interest for experiments with cold atomic gases. Various ramping procedures are used in experiment to transform one phase into another, and the available time for the process cannot be too long in order to avoid extrinsic losses. Whether some theoretical prediction is actually observable in experiment can thus depend in a subtle way on the unavoidable

when the ramp is infinitely slow. In contrast, such a system will pass through a series of well-defined non-thermal states in a quasistationary process. It would be interesting to see whether these states can be described by generalized Gibbs ensembles $\rho_{G}$ with constant entropy $\operatorname{Tr} \rho_{\mathrm{G}} \log \rho_{\mathrm{G}}$ (cf. Sec. 4.3). This should be investigated in the future. In the present chapter we consider only the case for which the system is initially in the ground state. 
excitation during the preparation of the state [193]. When the ramp duration is fixed to a given maximum value, it becomes important to find the optimal ramp though which a given point in parameter space can be reached through minimal excitation of the system [194, 195]. This question is closely related to the adiabatic behavior for $\tau_{d} \rightarrow \infty$, and could also be studied within DMFT for the Falicov-Kimball model or for the Hubbard model.

\subsection{Falicov-Kimball model: DMFT}

\subsubsection{Preliminary remarks}

Using nonequilibrium DMFT, the non-adiabatic excitation energy can be calculated for the Falicov-Kimball model (5.1) in the limit of infinite dimensions. In the following we assume that the system is in the ground state for times $t<0$. For $0 \leq t \leq \tau_{d}$ the hopping integral $V$ is changed according to a ramp protocol

$$
V(t)= \begin{cases}V_{i} & t \leq 0 \\ V_{i}+\Delta V r\left(t / \tau_{d}\right) & 0<t<\tau_{d} \\ V_{f}=V_{i}+\Delta V & t \geq \tau_{d},\end{cases}
$$

where $V_{i}$ is the initial hopping integral, $\tau_{d}$ is the total ramp time, $\Delta V$ is the ramp amplitude, and $r(x)$ is the ramp shape. The latter is a monotonously increasing function with $r(0)=0$ and $r(1)=1$. Similar ramps will be considered for the interaction strength. In the following, we use the semielliptic density of states (3.18), and focus on the homogeneous paramagnetic phase at half-filling. The energy scale is set by $V_{i} \equiv V \equiv 1$, and time is measured in units of $1 / V$.

The real-time Green functions can then be calculated from the numerical solution of Eqs. (5.7) and (5.11). When all hopping amplitudes are changes in time with a global factor $V(t)$, the the self-consistency for a semielliptic density of states is slightly modified with respect to Eq. (5.19) $:^{2}$

$$
\Lambda\left(t, t^{\prime}\right)=V(t) G\left(t, t^{\prime}\right) V\left(t^{\prime}\right) .
$$

The total energy $E(t)$ during the ramp is determined from Eq. (5.16). Because the Hamiltonian is time-dependent, $E(t)$ is not constant for $0 \leq t \leq \tau_{d}$. Finally, the excitation after the ramp is defined by the difference

$$
\Delta E\left(\tau_{d}\right)=E\left(\tau_{d}\right)-E_{0}\left(V_{f}\right),
$$

where $E_{0}\left(V_{f}\right)$ is the internal energy (5.16) in the ground state at hopping parameter $V_{f}$ and zero temperature.

\footnotetext{
2 This can be proven by mapping the Hamiltonian (5.1), with a time-dependent hopping integral $V(t)$ to a Hamiltonian $\tilde{H}$ with time-dependent interaction and constant hopping. In short: The action $\exp \left[-i \int d t H(t)\right]$ of the lattice model is invariant under a simultaneous scaling of the Hamiltonian $\tilde{H}(t)$ $=V H(t) / V(t)$ and transformation to new time variable $\tilde{t}(t)=\int_{0}^{t} d t^{\prime} V\left(t^{\prime}\right) / V$. By definition, $\tilde{H}$ has timedependent interaction $\tilde{U}(t)=U V / V(t)$ but constant hopping $V$, such that Eq. 3.19 is valid. Under the same transformation of time variables, the hybridization function $\Lambda\left(t, t^{\prime}\right)$ in the DMFT action (3.4) transforms as $\tilde{\Lambda}\left(t_{1}, t_{2}\right)=V\left(t_{1}\right) \Lambda\left(t_{1}, t_{2}\right) V\left(t_{2}\right) / V^{2}$, which proves the proposition.
} 


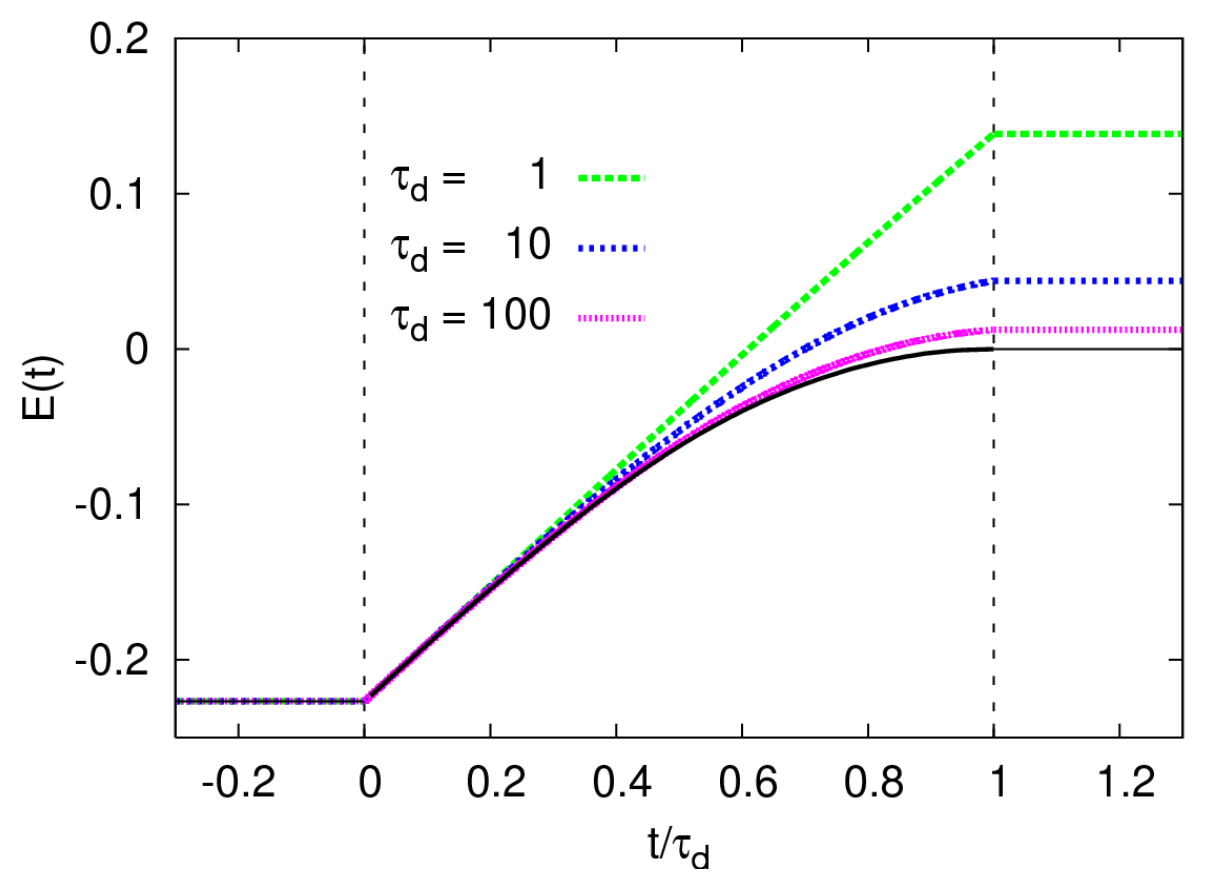

Figure 7.1: Internal energy $E(t)$ [Eq. (5.16)] during ramps $(7.2)$ of the hopping amplitude in the Falicov-Kimball model $\left[r(x)=x, U=1\right.$, and $\left.V_{f}=0\right]$, using various ramp durations $\tau_{d}$. For $t<0$ and $t>\tau_{d}$, the energy is constant. The solid black line is the internal energy $E(t)$ in the ground state at $U=1$ and hopping $V(t)$.

Eqs. (5.7), (5.11) and (7.3) can be written as a matrix version of Eq. (2.36), whose numerical solution requires only a minimal modification of the method presented in Sec. 2.5. In particular, only one nonlinear integro-differential equation is solved, and no explicit DMFT self-consistency iteration is needed. The Matsubara components $r^{\mathrm{M}}(\omega), q^{\mathrm{M}}(\omega)$, and $\lambda^{\mathrm{M}}(\omega)$ which enter the solution as initial condition for the real-time Green functions are obtained from the cubic equations (5.25).

Before presenting the results in the next subsection, a brief comment on the numerical algorithm is in order: To study the excitation energy (7.1) for large $\tau_{d}$ from the difference (7.4), the absolute energy (5.16), must be determined with an relative accuracy of the order of $\tau_{d}^{-\eta}$. On the other hand, the numerical error $\mathcal{E}$ due to time-discretization $\Delta t=$ $\left(\tau_{d} / N\right)$ with $N$ time-slices behaves as $\mathcal{E} \sim \Delta t^{p}=\left(\tau_{d} / N\right)^{p}$ for $\Delta t \rightarrow 0$, where $p$ depends on the algorithm used for the solution of the Volterra equation (2.53). Sufficient accuracy of the energy with respect to $\Delta E\left(\tau_{d}\right)$ thus requires $N \sim \tau_{d}^{1+\eta / p}$ timesteps for $\tau_{d} \rightarrow \infty$. On the other hand the Green function must be stored at $\mathcal{O}\left(N^{2}\right)$ time points and hence $N$ is the limiting numerical factor (we can go up to $N \approx 10000$ ). It is thus crucial to use an algorithm which is correct up to high-order in $\Delta t$ when the exponent $\eta$ is large. For ramps in the insulating phase, e.g., $\eta \geq 2$ is found (see below), and these results could not be obtained using the lowest order approximation for the differential and the integral in Eq. 2.53) $(p=1)$, but we have used higher order schemes instead $(p=5)$. 


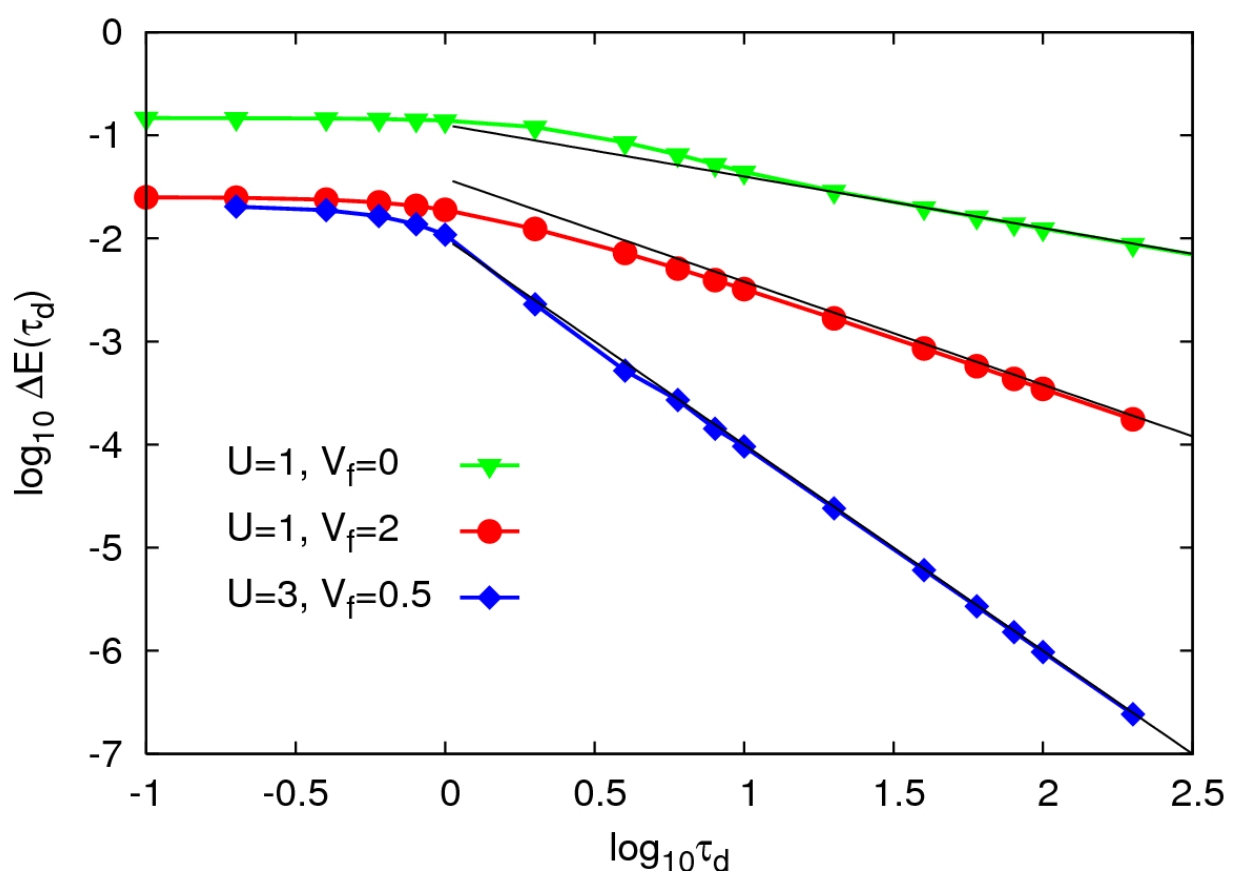

Figure 7.2: Excitation energy (7.4) after linear ramps of the hopping [Eq. (7.2), $r(x)=x$ ] within the metallic phase $\left[U=1, V_{f}=2\right]$, within the insulating phase $\left[U=3, V_{f}=0.5\right]$, and over the metal-insulator transition $\left[U=1, V_{f}=0\right]$. The energy scale is $V_{i} \equiv V=1$. The curves become independent of $\tau_{d}$ in the quench regime $\tau_{d} \lesssim 1 / V$. The solid black lines are linear fits $t \gtrsim 10$, with a slope $1 / 2,1$, and 2 (from top to bottom), corresponding to the asymptotic behavior (7.5).

\subsubsection{Results}

The time evolution of the energy (5.16) during a ramp (7.2) with linear shape $r(x)=x$ is plotted in Fig. 7.1. For small ramp-durations $\left(\tau_{d}=1\right)$, the energy raises linear with time. In this case, the system is essentially quenched, i.e., its state $|\psi(t)\rangle$ remains unchanged during the ramp, and the energy is thus only determined by the ramp protocol, $E(t) \approx$ $V(t) / V\left\langle\psi(0)\left|H_{k i n}\right| \psi(0)\right\rangle+\left\langle\psi(0)\left|H_{p o t}\right| \psi(0)\right\rangle$. In the opposite limit $\tau_{d} \rightarrow \infty$, the energy adiabatically follows the ground-state energy at hopping $V=V(t)$ (solid line in Fig. 7.1).

We now focus at the excitation $\Delta E\left(\tau_{d}\right)$ after the ramp, which is plotted in Fig. 7.2 for linear ramps $(7.2)$ within the gapless metallic phase $\left[U=1, V_{f}=2\right]$, within the gapped insulating phase $\left[U=3, V_{f}=0.5\right]$, and over the metal-insulator transition $[U=1$, $\left.V_{f}=0\right]$. The critical interaction is $U / V=2$. From Fig. 7.2 one can estimate the crossover timescale $\tau_{\text {quench}}$, which separates the regime in which the state of the system cannot follow the parameter change $\left(\tau_{d}<\tau_{\text {quench }}\right)$ from the "adiabatic regime" in which $\Delta E\left(\tau_{d}\right)$ decreases with increasing ramp-duration $\tau_{d}\left(\tau_{d}>\tau_{\text {quench }}\right)$. Independent of the ramp parameters, $\tau_{\text {quench }}$ turns out to be of the order of few times the inverse bandwidth. The decrease of $\Delta E\left(\tau_{d}\right)$ for $\tau_{d}>\tau_{\text {quench }}$ can be fitted with a power law (7.1) for $\tau_{d} \gtrsim 10$. The exponent appears to be a rational number, which depends only on the phase in which the system is before the ramp (metallic phase for $U<2$, insulating phase for $U>2$ ) and 
after the ramp (metallic phase for $U<2 V_{f}$, insulating phase for $U>2 V_{f}$ ). In summary, one has

$$
\Delta E\left(\tau_{d}\right) \stackrel{\tau_{d} \rightarrow \infty}{\sim} \begin{cases}\tau_{d}^{-\frac{1}{2}} & \text { linear ramp over met.-ins. transition } \\ \tau_{d}^{-1} & \text { linear ramp in metallic phase } \\ \tau_{d}^{-2} & \text { linear ramp in insulating phase } .\end{cases}
$$

The exponent $\eta=\frac{1}{2}$ for the excitation across the metal-insulator transition is independent of the ramp shape $r(x)$. A simple understanding of this exponent, similar as for ramps in exactly solvable quantum-critical systems [187, 188, 189], cannot be given at present. In particular, it should be clarified how this exponent is related to the critical behavior of equilibrium correlation functions, such as the density of states at the transition [174]. On the other hand we can give a simple explanation of Eq. (7.5) for ramps within the two phases. In particular we will see that the exponent $\eta=1$ is a consequence of the nonFermi-liquid behavior of the metallic phase in the Falicov-Kimball model, and that the excitation energy in the insulating phase depends strongly on the ramp, i.e., the exponent $\eta=2$ is not an intrinsic property of the Falicov-Kimball model.

\subsection{Perturbation theory for small ramps}

\subsubsection{Derivation}

Because the exponent in Eq. (7.5) and the crossover scale $\tau_{\text {quench }}$ do not depend on the ramp parameters $V_{i}$ and $V_{f}$ in (7.2) in detail, one can obtain important insight into the adiabatic behavior for ramps within the metallic and insulating phases of the FalicovKimball model from the limit of small ramp amplitudes $\Delta V \rightarrow 0$. In this section an expression for the $\Delta E\left(\Delta V, \tau_{d}\right)$ will be derived for $\Delta V \rightarrow 0$ from conventional first-order time-dependent perturbation theory.

The argument is not restricted to the Falicov-Kimball model and is thus formulated for the general Hamiltonian

$$
\begin{array}{r}
H(t)=H_{0}+\kappa(t) W \\
\kappa(t)=\Delta \kappa r\left(t / \tau_{d}\right),
\end{array}
$$

where $W$ is some operator, and $\kappa(t)$ is the ramp function that is characterized by the ramp amplitude $\Delta \kappa$, the ramp-duration $\tau_{d}$, and the ramp shape $r(x)$ as in Eq. (7.2). The excitation energy $\Delta E\left(\Delta \kappa, \tau_{d}\right)$ is now expanded in an power series in $\Delta \kappa$ for fixed $\tau_{d}$. For this purpose we expand the state $|\psi(t)\rangle$ in the instantaneous eigenbasis $\left|\phi_{n}(t)\right\rangle$ of the Hamiltonian (7.6) $\left[\epsilon_{n}(t)\left|\phi_{n}(t)\right\rangle=H(t)\left|\phi_{n}(t)\right\rangle\right]$,

$$
|\psi(t)\rangle=\sum_{n} a_{n}(t) e^{i \int d t^{\prime} \epsilon_{n}\left(t^{\prime}\right)}\left|\phi_{n}(t)\right\rangle
$$

where the phase factor is introduced for convenience. The Schrödinger equation reads

$$
i \frac{d}{d t} a_{n}(t)=\sum_{m} e^{i \int_{0}^{t} d t^{\prime} \epsilon_{n m}\left(t^{\prime}\right)}\left\langle\phi_{n}(t)\left|\frac{d}{d t}\right| \phi_{m}(t)\right\rangle,
$$


using the notation $\epsilon_{n m}(t)=\epsilon_{n}(t)-\epsilon_{m}(t)$. Eq. (7.9) must be solved with the initial condition $a_{m}(0)=\delta_{m 0}$, because the system is initially in the ground state $\left|\phi_{0}(0)\right\rangle$. For non-degenerate states $\left|\phi_{n}\right\rangle$ and $\left|\phi_{m}\right\rangle$ if follows from their definition that

$$
\begin{aligned}
\epsilon_{n m}(t)\left\langle\phi_{n}(t)\left|\frac{d}{d t}\right| \phi_{m}(t)\right\rangle & =\left\langle\phi_{n}(t)\left|H(t) \frac{d}{d t}-\frac{d}{d t} H(t)\right| \phi_{m}(t)\right\rangle \\
& =\left\langle\phi_{n}(t)\left|\frac{d H}{d t}\right| \phi_{m}(t)\right\rangle \\
& =\Delta \kappa \frac{r^{\prime}\left(t / \tau_{d}\right)}{\tau_{d}}\left\langle\phi_{n}(t)|W| \phi_{m}(t)\right\rangle .
\end{aligned}
$$

Hence Eq. (7.9) and the initial condition imply $a_{n}(t)=\delta_{n 0}+\mathcal{O}(\Delta \kappa)$, and thus, for $n \neq 0$,

$$
a_{n}(t)=\Delta \kappa \int_{0}^{t} \frac{d t^{\prime}}{\tau_{d}} r^{\prime}\left(\frac{t}{\tau_{d}}\right) \frac{\left\langle\phi_{n}\left(t^{\prime}\right)|W| \phi_{0}\left(t^{\prime}\right)\right\rangle}{\epsilon_{n 0}\left(t^{\prime}\right)} e^{i \int_{0}^{t^{\prime}} d t^{\prime \prime} \epsilon_{n 0}\left(t^{\prime \prime}\right)}+\mathcal{O}\left(\Delta \kappa^{2}\right) .
$$

This expression was already used in Ref. [187] as a starting point to discuss the excitation energy for ramps across quantum critical points. Here we proceed differently and study ramps which do not cross any critical point. The instantaneous eigenenergies $\epsilon_{n 0}(t)$ and eigenfunctions $\left|\phi_{n}(t)\right\rangle$ depend on $t$ only through the parameter $\kappa$, and we assume that an expansion around $\kappa=0$ is valid. This assumption breaks down at a critical point, but we consider only ramps within a given phase. Since $a_{n}(t)$ is already of order $\mathcal{O}(\Delta \kappa)$, we can thus replace $\epsilon_{n 0}(t)$ and $\left|\phi_{n}(t)\right\rangle$ by $\epsilon_{n 0} \equiv \epsilon_{n 0}(0)$ and $\left|\phi_{n}\right\rangle \equiv\left|\phi_{n}(0)\right\rangle$, respectively. Eq. 7.13 is then inserted into the excitation energy $\Delta E\left(\Delta \kappa, \tau_{d}\right)=\sum_{n \neq 0} \epsilon_{n 0}(t)\left|a_{n}(t)\right|^{2}$, which yields the final result

$$
\begin{gathered}
\Delta E\left(\tau_{d}, \Delta \kappa\right)=\Delta \kappa^{2} \int_{0}^{\infty} \frac{d \omega}{\omega} R(\omega) F\left(\omega \tau_{d}\right)+\mathcal{O}\left(\Delta \kappa^{3}\right) \\
R(\omega)=\sum_{n \neq 0}\left|\left\langle\phi_{n}|W| \phi_{0}\right\rangle\right|^{2} \delta\left(\omega-\epsilon_{n}\right) \\
F\left(\omega \tau_{d}\right)=\left|\int_{0}^{1} d s r^{\prime}(s) e^{i \omega \tau_{d} s}\right|^{2} .
\end{gathered}
$$

Here the correlation function $R(\omega)$ is independent of the ramp and can be interpreted as the density of possible excitations. Conversely, the "ramp spectrum" $F(x)$ does not depend on the Hamiltonian. Provided that the ramp is continuous, we have $F(x) \rightarrow 0$ for $|x| \rightarrow \pm \infty$, such that $F\left(\omega \tau_{d}\right)$ becomes increasingly peaked around $\omega=0$ for $\tau_{d} \rightarrow 0$. In fact, replacing $F\left(\omega \tau_{d}\right) \propto \tau_{d} \delta(\omega)$ is equivalent to Fermi's golden rule for $\left|a_{n}(t)\right|^{2}$. In turn, the non-adiabatic excitation (7.14) is due to deviations of $F\left(\omega \tau_{d}\right)$ from $\delta(\omega)$.

To quantify the main contributions to the integral (7.14), we split the integral at $\omega=C_{1} / \tau_{d}$, where $C_{1}$ is some constant. The contributions to (7.14) for $\omega<C_{1} / \tau_{d}$ and $\omega>C_{1} / \tau_{d}$ will be referred to as $\Delta E_{\text {center }}\left(\tau_{d}\right)$ and $\Delta E_{\text {tail }}\left(\tau_{d}\right)$, respectively. Furthermore, we make some rather general assumptions on the spectrum $R(\omega)$ : (i) Either $R(\omega)$ has a gap at $\omega=0$, or it can be expanded as a power series $R(\omega)=R_{1} \omega^{\nu}+\mathcal{O}\left(\omega^{\nu+1}\right)$ such that it vanishes at least linearly for $\omega \rightarrow 0$. (ii) $R(\omega) / \omega$ has a finite upper band edge $\Omega$ and 
is bounded from above, $R(\omega) \leq R_{2}$. The center contribution $\Delta E_{\text {center }}\left(\tau_{d}\right)$ can then be calculated by a simple change of variables in the integral,

$$
\begin{aligned}
& \Delta E_{\text {center }}\left(\tau_{d}\right) \equiv \Delta \kappa^{2} \int_{0}^{C_{1} / \tau_{d}} \frac{d \omega}{\omega} R(\omega) F\left(\omega \tau_{d}\right) \\
& \quad= \begin{cases}0 & R(\omega) \text { gapped at } \omega=0 \\
\Delta \kappa^{2} C_{2} \tau_{d}^{-\nu}+\mathcal{O}\left(1 / \tau_{d}^{\nu+1}\right) & R(\omega)=R_{1} \omega^{\nu}+\mathcal{O}\left(\omega^{\nu+1}\right)\end{cases}
\end{aligned}
$$

where the constant $C_{2}$ is given by

$$
C_{2}=R_{1} \int_{0}^{C_{1}} d x x^{\nu-1} F(x) .
$$

To estimate the tail contribution $\Delta E_{\text {tail }}\left(\tau_{d}\right)$ we use the upper bound $R_{2}$ for $R(\omega)$,

$$
\Delta E_{\text {tail }}\left(\tau_{d}\right) \leq \Delta \kappa^{2} R_{2} \int_{C_{1} / \tau_{d}}^{\Omega} d \omega F\left(\omega \tau_{d}\right)=\Delta \kappa^{2} \frac{R_{2}}{\tau_{d}} \int_{C_{1}}^{\Omega \tau_{d}} d x F(x)
$$

For $\tau_{d} \rightarrow \infty$, the integral in Eq. (7.20) is determined only by the asymptotic behavior of $F(x)$ at large frequencies $x$. When $F(x)$ vanishes exponentially for $x \rightarrow \infty$, then the excitation energy is also exponentially small. From the definition $(7.16)$ it follows that this is only the case if all derivatives of $r(x)$ vanish at $x=0$ and $x=1$. On the other hand, when $F(x)$ has an asymptotic expansion

$$
F(x)=\frac{f_{1}(x)}{x^{\nu}}+\mathcal{O}\left(1 / x^{\nu+1}\right)
$$

with some bounded function $f_{1}(x)>0$, then $\int_{C_{1}}^{\Omega \tau_{d}} d x F(x) \leq C_{3} \tau_{d}^{1-\nu}$ and $\Delta E_{\text {tail }}\left(\tau_{d}\right) \leq$ $\Delta \kappa^{2} C_{4} \tau_{d}^{-\nu}$ with constants $C_{3}$ and $C_{4}$. A similar bound from below is obtained, because $R(\omega)$ can always be estimated from below in some finite interval unless the bandwidth of $R(\omega)$ vanishes. Hence we have the general result

$$
\Delta E_{\text {tail }}\left(\tau_{d}\right) \propto \Delta \kappa^{2} \tau_{d}^{-\nu}+\mathcal{O}\left(1 / \tau_{d}^{\nu+1}\right)
$$

for a ramp spectrum which satisfies Eq. (7.21).

Eqs. (7.22) and (7.17) provide a direct expression for the large- $\tau_{d}$ asymptotic of the integral (7.14), $\Delta E\left(\tau_{d}\right)=\Delta E_{\text {center }}\left(\tau_{d}\right)+\Delta E_{\text {tail }}\left(\tau_{d}\right)$. Interestingly, the asymptotic behavior of the tail contribution 7.22 depends only on the ramp shape, while the asymptotic behavior of the center contribution (7.18) is an intrinsic property of the system. Note that the exponent $\nu$ in Eq. (7.21) is always larger or equal than two, provided that the ramp is smooth enough such that the derivative $r^{\prime}(x)$ in Eq. (7.16) does not diverge. Thus the asumptotic form of the excitation energy (7.14) for $\tau_{d} \rightarrow \infty$ is independent of the the ramp shape whenever the exponent $\nu$ in 7.18 is smaller than two. This already suggests that the relatively strong excitation for ramps in the metallic phase of the Falicov-Kimball model $[\eta=1$ in Eq. (7.5)] does not depend on the ramp shape but is due the contribution intrinsic $\Delta E_{\text {center }}\left(\tau_{d}\right)$. In the following two subsections this will be further substaintiated. 

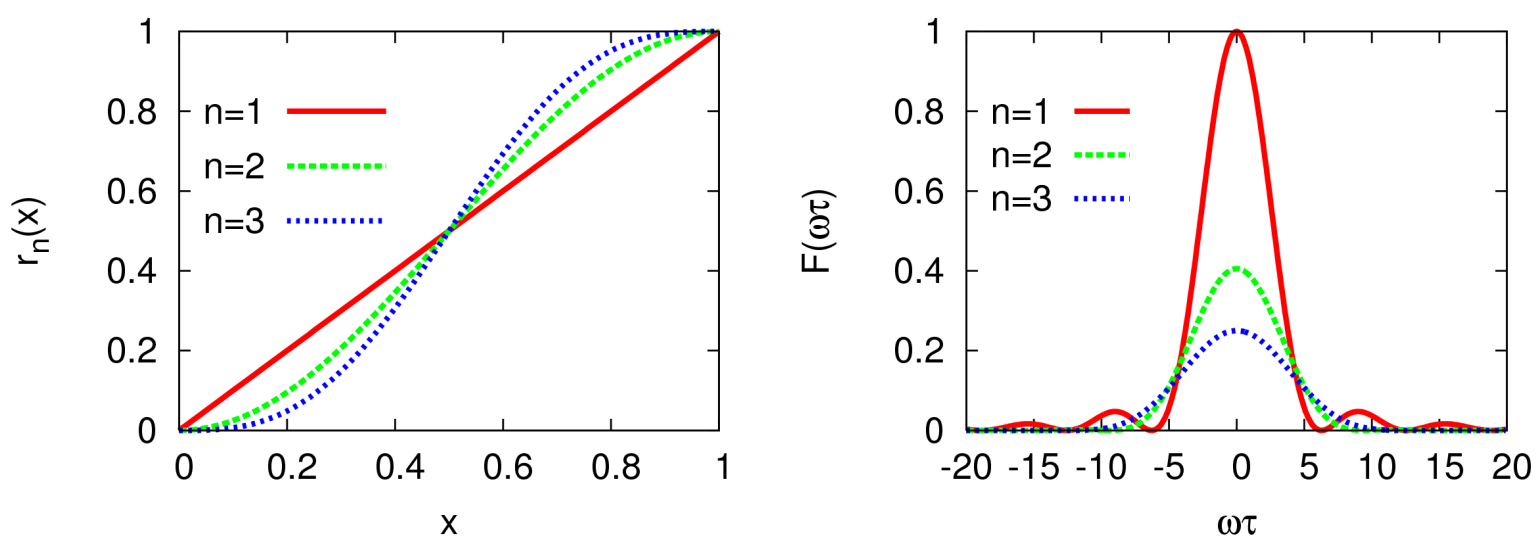

Figure 7.3: Right: The ramps shapes (7.23), and the corresponding ramp spectra (7.24). The Fresnel oscillations in $F_{1}(\omega)$ are due to the discontinuity in the first derivative of $r_{1}(x)$ at $x=0$ and $x=1$.

Furthermore, Eq. (7.14) is not restricted to the asymptotic behavior. It could be used to get a lowest-order approximation for an optimal ramp with least excitation energy, in terms of the spectrum $R(\omega)$. In addition, one can directly read off the crossover scale

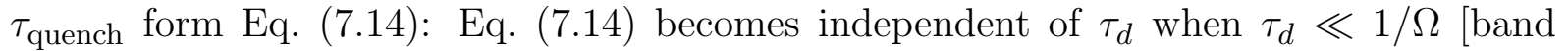
width of $R(\omega)$ ], such that $F\left(\omega \tau_{d}\right)=F(0)+\mathcal{O}\left(\omega^{2} \tau_{d}^{2}\right)$ is approximately constant for $\omega<\Omega$.

\subsubsection{Insulating phase}

In the gapped phase, the center component (7.18) to the excitation energy vanishes, and the excitation energy is completely determined by the asymptotic behavior of $F(x)$ in Eq. 7.20). This suggests that the excitation energy strongly depends of the ramp shape for ramps in the insulating phase of the Falicov-Kimball model. To show this we perform DMFT calculations for ramps in the insulating phase with various ramp shapes,

$$
\begin{aligned}
& r_{1}(x)=x \\
& r_{2}(x)=\frac{1-\cos (\pi x)}{2} \\
& r_{3}(x)=\frac{\pi x-\cos (\pi x) \sin (\pi x)}{\pi},
\end{aligned}
$$

which are defined by $r_{n}^{\prime}(x) \propto \sin ^{n}(\pi x)$, such that the $n$ first derivatives of $r_{n}(x)$ vanish at $x=0$ and $x=1$ (Fig. 7.3). The latter ensures $F_{n} \sim x^{-2 n}$ for $x \rightarrow \infty$,

$$
\begin{aligned}
& F_{1}(\omega)=2 \frac{1-\cos (\omega)}{\omega^{2}} \\
& F_{2}(x)=\frac{\pi^{4}}{2} \frac{1+\cos (\omega)^{2}}{\left(\pi^{2}-\omega^{2}\right)^{2}} \\
& F_{3}(x)=32 \pi^{4} \frac{1-\cos (\omega)}{\omega^{2}\left(4 \pi^{2}-\omega^{2}\right)^{2}} .
\end{aligned}
$$




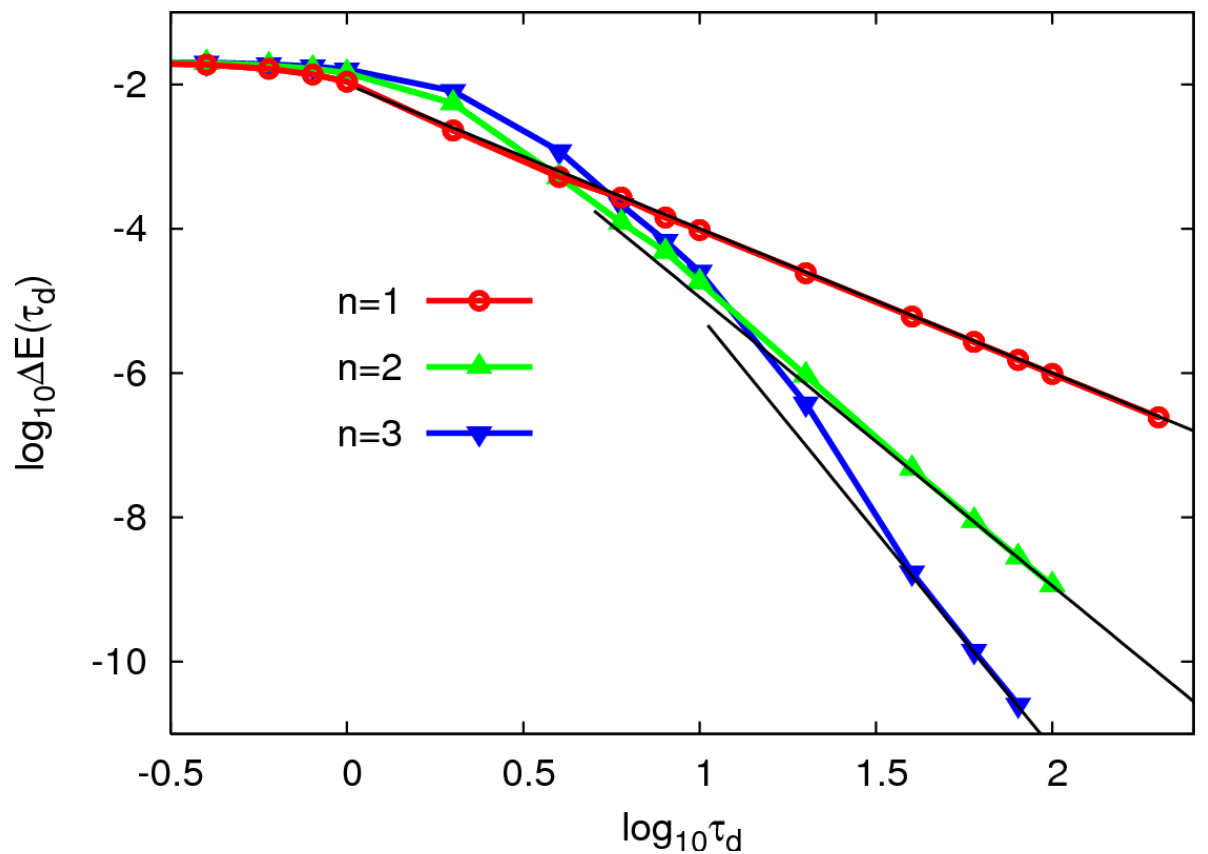

Figure 7.4: Same as Fig. 7.2, for ramps within the insulating phase. $r(x)=r_{n}(x)$ $[\mathrm{Eq} 7.23], U=3, V_{f}=0.5$. The black linear lines correspond to power law behavior (7.1) with $\eta=2(n=1)$ and $\eta=4(n=2)$, and $\eta=6(n=3)$.

Because the intrinsic contribution (7.17) to the excitation vanishes for gapped phases, one obtains from Eqs. (7.22) and (7.24) the excitation after ramps $r_{n}(x)$ is given by $\Delta E\left(\tau_{d}\right)$ $\sim \Delta V^{2} \tau_{d}^{-2 n}$ to lowest order in the ramp amplitude. The excitation energy after ramps (7.23) in the insulating phase of the Falicov-Kimball model is plotted in Fig. 7.4. In fact, the curves can be fitted with power laws 7.4 for large $\tau_{d}$, and the exponents are given by $\eta=2$ for the linear ramp (7.23a) and $\eta=4$ for the ramp $7.23 \mathrm{~b}$ ), respectively. For the ramp (7.23c), the results are consistent with $\eta=6$, but the excitation energy is too small for a power law fit in the accessible range.

Because no explicit form for $R(\omega)$ is known for the Falicov-Kimball model in the insulating phase, we cannot directly compare Eq. (7.14) with DMFT results for $\tau_{d}<\infty$. However, in the atomic limit $(V=0)$ at half-filling, $R(\omega)$ consists of a single delta-function peak at $\omega=U]^{3}$ When $R(\omega) \propto \delta(\omega-U)$ is inserted into Eq. (7.14), one obtains

$$
\Delta E\left(\tau_{d}\right) \propto F\left(U \tau_{d}\right) .
$$

In fact, from Fig. 7.5 one can see that the Fresnel oscillations of $F_{1}(x)$ [Fig. 7.3] are indeed apparent in the excitation energy for ramps between states with $V \ll U$. In the limit $\tau_{d}$ $\rightarrow \infty$, oscillations become washed out because $R(\omega)$ has a finite bandwidth $\Omega$ for $V>0$, such that the integral (7.14) averages over many oscillations for $\tau_{d} \gg 1 / \Omega$.

\footnotetext{
${ }^{3}$ In the atomic limit one should rather consider a ramp of the interaction strength $U$, such that the operator $W$ in Eq. 7.6 is given by the double occupancy, and $R$ can be evaluated directly from the definition (7.15). For finite $V$, an interaction ramp and a ramp of the hopping amplitude can be mapped onto each other, as explained in footnote 2 on page 102 .
} 


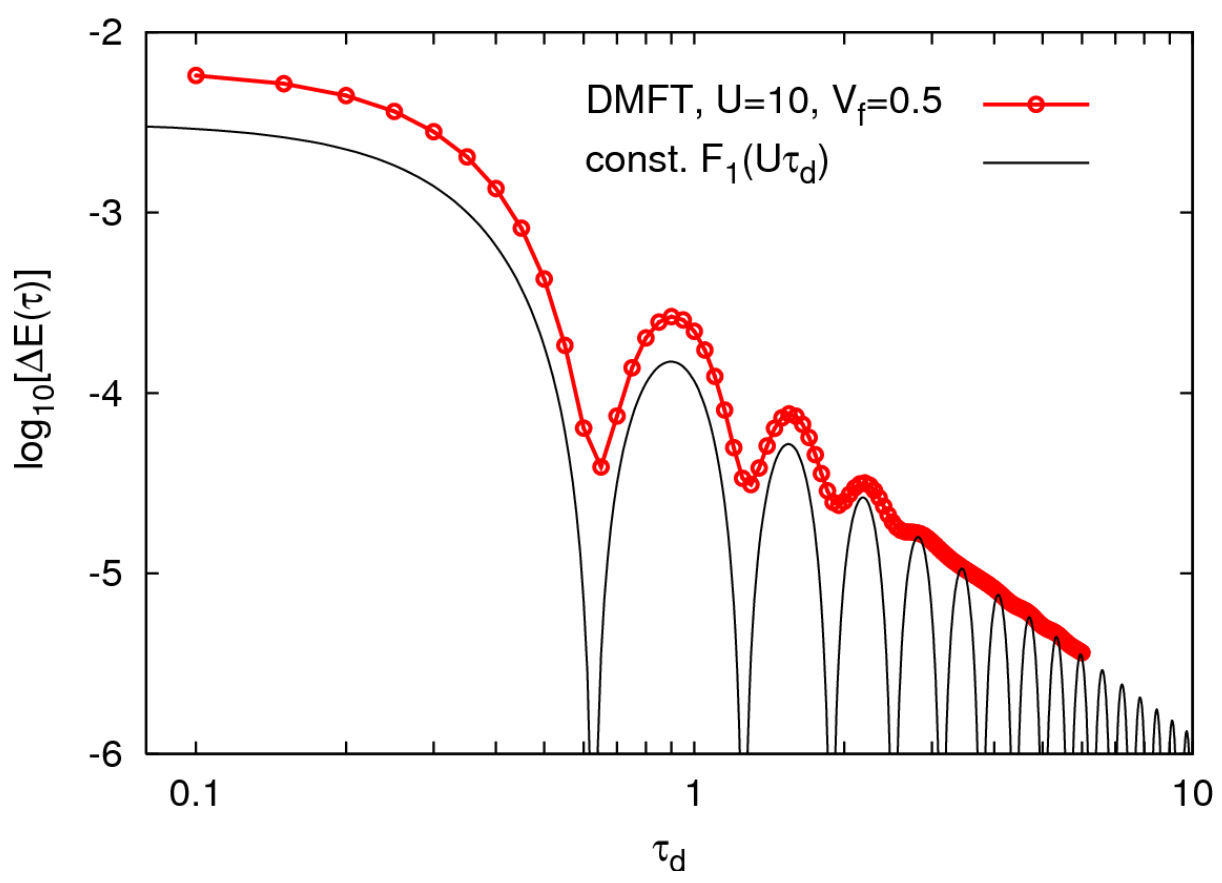

Figure 7.5: Excitation energy 7.4 after the linear ramp $7.23 \mathrm{a})$ of the hopping within the insulating phase $\left[U=10, V_{f}=0.5\right]$, compared to Eq. 7.25$)$ for small ramp amplitudes $\left[F_{1}\right.$ given by Eq. $\left.\left.7.24 \mathrm{a}\right)\right]$.

In conclusion, the perturbative expression (7.14) can explain the general features of the DMFT results for ramps within the insulating phase of the Falicov-Kimball model. Although there is a gap in the spectrum, the excitation energy is not exponentially small, but instead both the asymptotic behavior for $\tau_{d} \rightarrow \infty$ and also some intermediate time features are not universal for the Falicov-Kimball model, but depend on the ramp shape $r(x)$ in detail.

\subsubsection{Metallic phase}

For a ramp of the interaction strength in the Hubbard model and Falicov-Kimball model, the operator in Eq. (7.6) is given by the double occupancy ${ }^{4}$

$$
W=\sum_{i} n_{i \downarrow} n_{i \uparrow}=\sum_{\boldsymbol{k}, \boldsymbol{k}^{\prime}, \boldsymbol{q}} c_{\boldsymbol{k}+\boldsymbol{q} \downarrow}^{\dagger} c_{\boldsymbol{k} \downarrow} c_{\boldsymbol{k}^{\prime}-\boldsymbol{q} \uparrow}^{\dagger} c_{\boldsymbol{k}^{\prime} \uparrow} .
$$

The excitation density $R(\omega)$ can be evaluated at $U=0$. This can be used to compute the excitation $\Delta E\left(\tau_{d}\right)$ for ramps of the interaction parameter $U$ from $U=0$ to $U=\Delta U$ up to order $\mathcal{O}\left(\Delta U^{2}\right)$ from Eq. (7.14), and compare to the DMFT results.

For this purpose we introduce the the imaginary-time ordered correlation function

$$
\left.\tilde{R}\left(\tau-\tau^{\prime}\right)=\left\langle T_{\tau} W(\tau) W\left(\tau^{\prime}\right)\right]\right\rangle_{0},
$$

\footnotetext{
${ }^{4}$ In this section we use a unified notion for the Hubbard model and the Falicov Kimball model: For the Falicov-Kimball model, spin $\uparrow$ is immobile.
} 

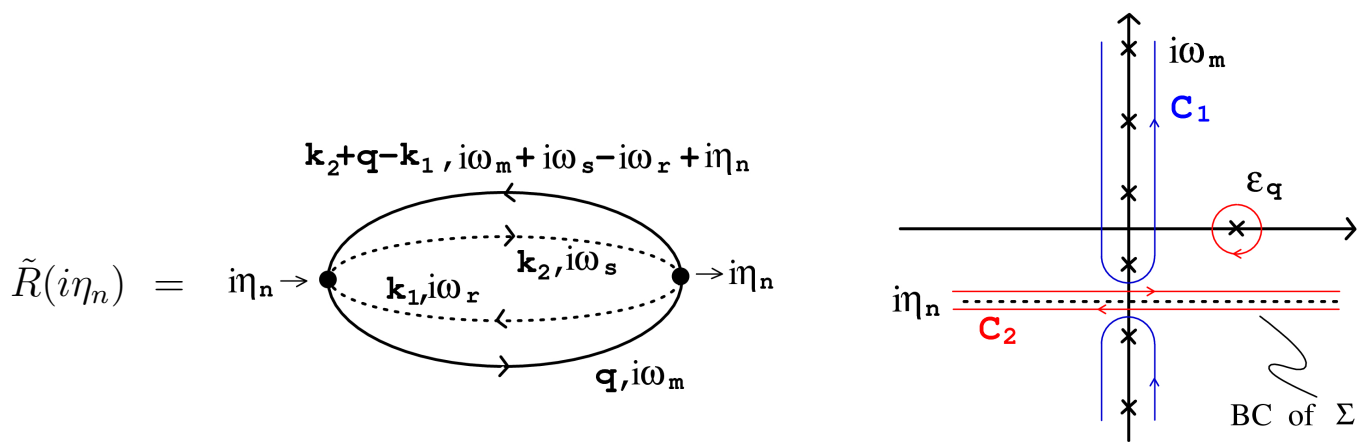

Figure 7.6: (a) Diagrammatic representation of Eq. (7.29). Lines represent the noninteracting momentum-resolved Green function $g_{\boldsymbol{q} \sigma}^{0}\left(i \omega_{m}\right)=1 /\left(i \omega_{m}-\epsilon_{\boldsymbol{q} \sigma}\right)$ for $\sigma=\uparrow$ (solid lines) and $\sigma=\downarrow$ (dashed lines). Momentum is conserved at the vertices, frequency $i \eta_{n}$ enters at the left vertex. (b) Transformation of the Matsubara sum (7.31) to the real frequency intergal 7.32 , using the usual expression $\sum_{i \omega_{m}} w\left(i \omega_{m}\right)=(i \beta / 2 \pi) \oint_{C_{1}} d z f(z) w(z)$, where $f(z)$ is the Fermi function and $w(z)$ is some analytic integrand. The integrand in Eq. (7.31) has a branch cut at $z=-i \eta_{n}$ due to the branch cut of $\Sigma(z)$ along the real axis, and a pole at $\epsilon_{\boldsymbol{q} \uparrow}$. Then the contour $C_{1}$ is transformed into $C_{2}$, which yields (7.31), using that the Fermi function is periodic under shift with bosonic Matsubara frequencies, $f\left(\omega-i \eta_{n}\right)=f(\omega)$.

where the expectation value $\langle\cdot\rangle_{0}=\operatorname{Tr}\left[e^{-\beta H_{0}} \cdot\right] / \operatorname{Tr}\left[e^{-\beta H_{0}}\right]$ is taken in the noninteracting state at temperature $T=1 / \beta$ (the limit $T \rightarrow 0$ is taken at the end), and $W$ is given by (7.26). ( $T_{\tau}$ is the imaginary time ordering operator). The interaction operator contains an even number of Fermi operators, and hence $\tilde{R}(\tau)$ satisfies periodic boundary conditions on the imaginary time contour $\tau \in[0, \beta]$ and can be expanded in bosonic Matsubara frequencies $\eta_{n}=2 \pi n / T, \tilde{R}\left(i \eta_{n}\right)=\int_{0}^{\beta} d \tau \tilde{R}(\tau) e^{-i \eta_{n} \tau}$. Using the Lehmann representation one can show that $\tilde{R}\left(i \eta_{n}\right)$ is related to $R(\omega)$,

$$
R(\omega)=-\frac{1}{\pi} \operatorname{Im} \tilde{R}(\omega+i 0)
$$

where $\tilde{R}(\omega+i 0)$ is the unique analytical continuation of $\tilde{R}\left(i \eta_{n}\right)$ from $\eta_{n}>0$ to the upper half of the complex frequency plane.

To calculate $\tilde{R}$, the expectation value $(7.27)$ is factorized using Wicks theorem [Eq. 2.34], and transformed to Matsubara frequencies. It turns out that the only nonvanishing contractions for $\eta_{n} \neq 0$ is given by

$$
\tilde{R}\left(i \eta_{n}\right) \stackrel{n \neq 0}{=} \sum_{\boldsymbol{k}_{1}, \boldsymbol{k}_{2}, \boldsymbol{q}} \sum_{r, s, m} g_{\boldsymbol{k}_{2} \uparrow}^{0}\left(i \omega_{s}\right) g_{\boldsymbol{k}_{1} \uparrow}^{0}\left(i \omega_{r}\right) g_{\boldsymbol{q}_{\downarrow}}^{0}\left(i \omega_{m}\right) g_{\boldsymbol{k}_{2}-\boldsymbol{q}-\boldsymbol{k}_{1}, \downarrow}^{0}\left(i \omega_{m}+i \omega_{s}-i \omega_{r}+i \eta_{n}\right),
$$

where $g_{\boldsymbol{q} \sigma}^{0}\left(i \omega_{m}\right)=1 /\left(i \omega_{m}-\epsilon_{\boldsymbol{q} \sigma}\right)$ is the noninteracting Green function at momentum $\boldsymbol{q}$, and $i \omega_{m}$ are fermionic Matsubara frequencies. The integral has a simple diagrammatic representation (Fig. 7.6 a). The diagram is split into one Green function line $g_{\boldsymbol{q} \downarrow}^{0}\left(i \omega_{m}\right)$ and 
the remainder, which we identify as the second-order self-energy contribution $\Sigma_{\boldsymbol{q} \downarrow}^{(2)}$,

$$
\tilde{R}\left(i \eta_{n}\right)=\sum_{\boldsymbol{q}} \sum_{i \omega_{m}} g_{\boldsymbol{q} \downarrow}^{0}\left(i \omega_{m}\right) \Sigma_{\boldsymbol{q} \downarrow}^{(2)}\left(i \eta_{n}+i \omega_{m}\right) .
$$

One can now transform the Matsubara summation into a frequency integral, where it must be taken into account that the self-energy $\Sigma(z)$ has a branch cut along the real axis with $\Sigma(\omega+i 0)=\Sigma(\omega-i 0)^{*}$ (Fig. 7.6b). The result is

$$
\tilde{R}\left(i \eta_{n}\right)=\sum_{\boldsymbol{q}}\left\{f\left(\epsilon_{\boldsymbol{q} \uparrow}\right) \Sigma_{\boldsymbol{q} \uparrow}^{(2)}\left(i \eta_{n}+\epsilon_{\boldsymbol{q} \uparrow}\right)-\frac{1}{\pi} \int d \omega f(\omega) \frac{\operatorname{Im} \Sigma_{\boldsymbol{q} \uparrow}^{(2)}(\omega+i 0)}{\omega-i \eta_{n}-\epsilon_{\boldsymbol{q} \uparrow}}\right\},
$$

where $f(\epsilon)$ is the Fermi function. Eq. (7.31) is continued to the real frequencies by replacing $i \eta_{n} \rightarrow \omega+i 0$, and the spectrum (7.28) is obtained as

$$
R(\omega)=\frac{1}{\pi} \sum_{\boldsymbol{q}}\left[f\left(\omega+\epsilon_{\boldsymbol{q} \downarrow}\right)-f\left(\epsilon_{\boldsymbol{q} \downarrow}\right)\right] \operatorname{Im} \Sigma_{\boldsymbol{q} \downarrow}^{(2)}\left(\omega+\epsilon_{\boldsymbol{q} \downarrow}\right) .
$$

To compare the results with DMFT we go to the limit of infinite dimensions where the self-energy is independent of $\boldsymbol{q}$. (The following considerations can be generalized to a $\boldsymbol{q}$-dependent $\Sigma$, and the final conclusions are the same.) The $\boldsymbol{q}$-summation is replaced by an integral over the density of states. Furthermore we can take the limit of $T \rightarrow 0$, which yields the final result

$$
R(\omega)=\frac{1}{\pi} \int_{0}^{\omega} d \epsilon \rho_{\downarrow}(\epsilon) \operatorname{Im} \Sigma_{\downarrow}^{(2)}(\epsilon+\omega)
$$

for the spectrum 7.15 in the Hubbard or Falicov-Kimball model in the limit of infinite dimensions at $U=0$.

The 2nd order self-energy for the Falicov-Kimball model can be obtained from Eq. (5.25) and $(5.26)$,

$$
\frac{1}{\pi} \operatorname{Im} \Sigma_{\mathrm{FKM}}^{(2)}(\omega)=\left(1-n_{f}\right) n_{f} \rho(\omega) \stackrel{\omega \rightarrow 0}{\sim}\left(n_{f}-1\right) n_{f} \rho(0) .
$$

For the Hubbard model, it is given by [56]

$$
\frac{1}{\pi} \operatorname{Im} \Sigma_{\text {Hubbard }}^{(2)}(\omega)=\int_{0}^{\omega} d \mu \rho(\mu-\omega) \int_{0}^{\mu} d \nu \rho(\nu) \rho(\mu-\nu) \stackrel{\omega \rightarrow 0}{\sim} \frac{\rho(0)^{3}}{2} \omega^{2},
$$

according to the rules of Sec. 2.4. The different behavior of the self-energy at small $\omega$ is due to the fact that the metallic state of the Hubbard model is a Fermi liquid, but not for the Falicov-Kimball model, where the scattering rate is always finite even for electrons at the Fermi energy. Eqs. (7.35) and (7.34) can then be inserted in Eq. (7.33), which in turn can be used to calculate the asymptotic behavior $(7.18)$ of the intrinsic contribution $\Delta E_{\text {center }}\left(\tau_{d}\right)$ to Eq. (7.14),

$$
\begin{aligned}
\text { Hubbard: } & R(\omega) \stackrel{\omega \rightarrow 0}{\sim} \frac{\rho(0)^{4}}{6} \omega^{3} & \Rightarrow \Delta E_{\text {center }}\left(\tau_{d}\right) \propto \tau_{d}^{-3} \\
\text { Falicov-Kimball: } & R(\omega) \stackrel{\omega \rightarrow 0}{\sim}\left(1-n_{f}\right) n_{f} \rho(0)^{2} \omega & \Rightarrow \Delta E_{\text {center }}\left(\tau_{d}\right) \propto \tau_{d}^{-1} .
\end{aligned}
$$




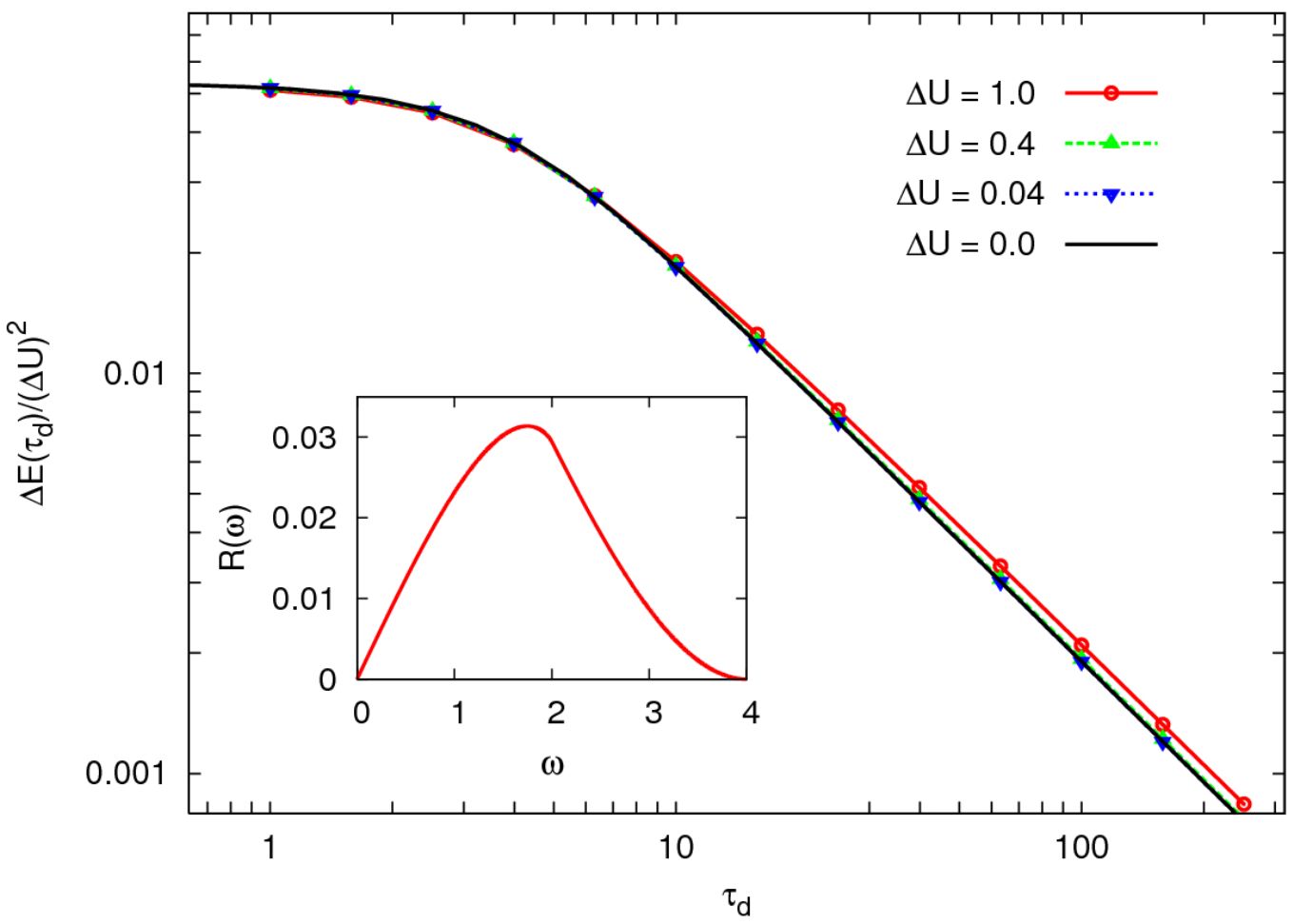

Figure 7.7: Excitation $\Delta E\left(\tau_{d}\right)$ in the Falicov-Kimball model, after gaussian ramps [Eq. (7.38), $\left.c_{2}=36\right]$ from interaction $U=0$ to $\Delta U$. The curve $\Delta U=0$ is obtained from Eq. (7.14), where $R(\omega)$ is obtained from Eqs. (7.33) and (7.34), and $F(\omega)$ is given by Eq. (7.38). The excitation spectrum $R(\omega)$ is plotted in the inset.

On the other hand, the tail contribution $\Delta E_{\text {tail }}\left(\tau_{d}\right)$ is at most $\Delta E_{\text {tail }}\left(\tau_{d}\right) \sim \tau_{d}^{-2}$ for $\tau_{d} \rightarrow \infty$ (see above). Consequently, the total excitation energy (7.14) is governed by the intrinsic contribution for the Falicov-Kimball model, whereas for the Hubbard model tail contribution is larger than the intrinsic contribution for linear ramps. Eq. (7.37) is in agreement with the numerical finding (7.5) for ramps in the metallic phase, which we can therefore understand as a consequence of the fact that the metallic state is not a Fermi liquid.

Since $R(\omega)$ is exactly known for the Falicov-Kimball model at $U=0$ from Eqs. (7.33) and (7.34), one can evaluate $\Delta E_{1}\left(\tau_{d}\right)$ [Eq. (7.14)] for arbitrary interaction ramps $U(t)=$ $\Delta U r(x)$, and compare to the corresponding DMFT result $\Delta E\left(\tau_{d}\right)$. In Fig. 7.7 this is done for a "Gaussian ramp", defined by

$$
r^{\prime}(x)=c_{1} \exp \left[-c_{2}(x-1 / 2)^{2}\right], \quad F(x)=\frac{\pi c_{1}^{2}}{c_{2}} \exp \left(-\frac{x^{2}}{4 c_{2}}\right)
$$

where $c_{1}$ is a normalization constant to satisfy $\int_{0}^{1} d x r^{\prime}(x)=r(1)-r(0)=1$, and $c_{2}$ is chosen such that $r^{\prime}(x)$ is sufficiently small for $x \notin[0,1]$, i.e., the expression for $F$ holds up to terms which are exponentially small in $c_{2}$. The agreement is very good for $\Delta U \leq 1$ and $\tau_{d}$ ranging from the quench regime $\left(\tau_{d} \rightarrow 0\right)$ to the asymptotic regime $\left(\tau_{d} \rightarrow \infty\right)$, 
where $\Delta E\left(\tau_{d}\right) \sim 1 / \tau_{d}$. Similar as for the insulating phase, this corroborates the simple perturbative expression which (7.14) can explain the non-adiabatic excitation. 


\section{Part III}

\section{Time-resolved spectroscopy on solid-state systems}




\section{Chapter 8}

\section{Time-resolved spectroscopy on correlated systems}

\subsection{Pump-probe experiments}

In pump-probe experiments a sample is excited by a short and intense laser pulse (the pump-pulse), and a second pulse (the probe-pulse) is used to characterize the excited state after a controlled time delay. The pump-probe setup has been used for more than two decades to investigate the dynamics of chemical reactions [196] and of excited states in semiconductors [197] and metals [198. The accessible time resolution is of the order of few femtoseconds, and both optical and photoemission spectroscopy can be used as a probe technique. In 1999, the Nobel prize for chemistry was awarded to Ahmed H. Zewail for "for his studies of the transition states of chemical reactions using femtosecond spectroscopy ". More recently, such time-resolved experiments have been used to record various nonequilibrium processes in strongly correlated materials, including those induced by the Coulomb interaction between electrons, or the scattering of electrons on defects and phonons [199, 200, 201, 202, 203, 204, 205, 206, 207, 208. Those results are particulary interesting for systems which are close to a phase transition, where many degrees of freedom contribute to the dynamics on very different time scales.

Since the time delay between pump and probe is usually under good control, the bottleneck for a good time-resolution consists in the generation of short and intense laser pulses. Recent advances in femtosecond laser techniques [209, 210] have enabled the generation of sub 10 spulses in the visible and infrared, which are sufficiently short to resolve the real-time dynamics of correlated electrons in solids. Some solid-state systems have been investigated even on the attosecond time scale [211], although these experiments have not yet been performed in the pump-probe setup as described above. In addition, free-electron lasers such as the FLASH facility in Hamburg [212] can generate pulses in the extreme ultraviolet to soft x-ray regime with a duration of only few femtoseconds, and thus enable the investigation of the combined electron-lattice dynamics with unprecedented resolution.

Quite often it is the goal of those nonequilibrium solid-state experiments to distinguish the electronic dynamics from that of other degrees of freedom. Several pump-probe 
experiments on correlated systems directly address the interplay between electronic and lattice degrees of freedom, e.g., at the metal-insulator transition in $\mathrm{VO}_{2}$ [213, 214], during the nonthermal melting of crystals after a pump excitation of the electron gas [215], or through pump excitation of coherent phonon modes [202, 216]. When the coupling between electrons ans phonons is weak, the relaxation of the electron-lattice system can be described within the two-temperature model [217]. In this picture the electron gas reaches thermal equilibrium after a short relaxation time, but electronic and lattice temperature are then still different from each other. The temperature deviations level out through a slow heat exchange between the two subsystems. In correlated systems, a state in which the electronic temperature $T_{e}$ is higher than the lattice temperature $T_{l}$ can be quite distinct from the equilibrium state of the system at temperature $T_{e}$, i.e., regions af the electronic phase diagram become accessible which can never be reached in equilibrium experiments [204, 205, 218.

Nevertheless, the two-temperature model is still a quasiequilibrium description. The true dynamics of the electronic system has so far been observed mainly for simple metals and semiconductors, because thermalization is apparently much faster in strongly correlated systems. For example, the thermalization of pump-excited electron distributions due to electron-electron scattering has been investigated with time-resolved photoemission [219], and the buildup of screening in the electron-hole plasma in GaAs was studies with terahertz time-resolved optical spectroscopy [220, 221]. On the other hand, resolution of the electronic dynamics would be particulary interesting in correlated systems, such as materials close to the Mott metal-insulator transition which is driven by the Coulomb interaction between electrons moving in a crystal lattice. A new perspective on this phenomenon would be obtained if one could, e.g., monitor the formation of welldefined quasiparticles in a metallic state after a pump excitation of the insulator, and thus observe how the Mott transition happens in time. Up to now several Mott and charge-transfer insulators have been driven to an intermediate metallic state by a laser pump pulse [199, 200, 203, 204, 205]. These experiments have focused on the reformation of the insulating state, which presumably happens due to a coupling of the valence band electrons to slower degrees of freedom. However, also the much faster buildup of the metallic state, which is most likely a purely electronic process, has been resolved recently through the reflectivity change during the pump-induced transition from the insulator to a metal in an organic charge-transfer component. It happens on the timescale of the inverse hopping amplitude, on which the response of the lattice can be neglected.1]

\section{Theoretical description within DMFT}

For the theoretical description of these pump-probe experiments we must understand both the effect of the pump pulse on the sample, and the probe of the nonequilibrium state. Nonequilibrium DMFT can serve this purpose [72, 73]. The main purpose of this Chapter is a detailed description of the probe process for time-resolved optical and photoemission spectroscopy in terms of the linear response of the pump-induced nonequilibrium state to

\footnotetext{
${ }^{1}$ Unpublished results were presented by A. Cavalleri during the Korrelationstage workshop in Dresden, March 2009.
} 
a weak time-dependent electromagnetic field. Before doing this, we will briefly comment on the theoretical description of the pump process.

Throughout the chapter we restrict ourselves to situations for which the nuclear motion can be neglected to first approximation, which holds on sufficiently short timescales because electrons usually response much faster than the lattice. In future, it may be possible to incorporate also the lattice dynamics into a nonequilibrium DMFT setup in principle. In fact, DMFT for equilibrium it has already been demonstrated that electronic correlations can have a profound influence on the crystal structure [222]. The combined analysis of electron and lattice dynamics would be very interesting in view of the experimental advances mentioned above, but this topic is beyond the scope of this thesis.

\section{Modelling of the pump pulse}

When the overlap of pump and probe pulse in the sample is not the main focus of the experiment, one may describe the excited state right after the pump pulse by a density matrix which is taken to be the initial state for the subsequent relaxation. A simple way to construct such a density matrix is to take the equilibrium state of a different Hamiltonian. Note that the latter is thereby only a way to parameterize the density matrix. In effect, this approach replaces the complicated pump-induced dynamics by a quantum quench, and we will use this concept in Sec. 8.4 to illustrate the general results for the probe process.

In many cases, such a description of the pump pulse can give a first understanding of the excited state which is more intuitive than an explicit calculation of the pumpexcitation. This idea can be illustrated by two of the experiments mentioned above: (i) In the photoinduced metal-insulator transition, the Mott insulating state is excited via "photodoping electrons from the lower to the upper Hubbard band". This process is nothing but the injection of pairs of doubly occupied and empty sites in the stronglyrepulsive insulator. The simplest way to model this state is through a metallic state (the equilibrium state of the Hubbard model at small $U$ ), while the relaxation dynamics is governed by a Hubbard model in the strongly repulsive regime. (ii) In the experiment on GaAs [220, 221], the pump pulse excites electrons from the valence band into the conduction band, and the subsequent relaxation of the electron-hole plasma due to the Coulomb interaction is observed by reflection measurements. The recombination of electrons and holes takes much longer and is not studied in the experiment. Directly after the pump pulse, the particles can be considered to be uncorrelated because the pump is too short to allow the build-up of Coulomb correlations. Consequently, the results of this experiment have been compared to the time evolution the Coulomb gas after a quench from the noninteracting state to weak interactions [223, 224].

Clearly the reduction of the of the pump-process to a idealized initial state can only lead to qualitative results. Although many aspects of the dynamics after a quench, such as the formation of long-lived quasistaionary states or the occurance of dynamical transitions (Ch. 6) are probably be rather independent of the specific form of the initial state, a microscopic modelling of the pump pulse will be desirable at some point. In principle such 
a description of the excitation process is accessible within DMFT through the introduction of Peierls factors [Eq. (1.2)] in the hopping amplitudes [43, 68, 70].

\subsection{Optical spectroscopy}

\subsubsection{Time-resolved optical spectroscopy}

To describe the experimental signals of time-resolved optical spectroscopy one has to know how electromagnetic pulses of finite length propagate through a material which is driven out of equilibrium by means of the pump pulse [225, 226, 227]. When the probe field is weak, its effect can be calculated as a linear response $\delta \boldsymbol{j}$ of the current $\boldsymbol{j}$ to the external electrical field $\delta E_{\beta}(\boldsymbol{r}, t)$,

$$
\delta j_{\alpha}(\boldsymbol{r}, t)=\int_{-\infty}^{t} d t^{\prime} \sigma_{\alpha \beta}\left(t, t^{\prime}\right) \delta E_{\beta}\left(\boldsymbol{r}, t^{\prime}\right) .
$$

Here and in the following $\alpha$ and $\beta$ denote cartesian components of the vectors, and repeated indices are summed over. Eq. (8.1) defines the optical conductivity $\sigma_{\alpha \beta}\left(t, t^{\prime}\right)$ for samples that are not in equilibrium. Of course $\sigma\left(t, t^{\prime}\right)$ must be causal, i.e., it vanishes for $t<t^{\prime}$.

It is important to note that only the response $(8.1)$ is linear in the probe field $\delta E_{\beta}\left(\boldsymbol{r}, t^{\prime}\right)$, whereas the electric pump fields that act on the sample can be arbitrarily strong. Furthermore, we have assumed that the linear response relation (8.1) is essentially local in space. This assumtion is valid when the wavelength is much larger than the lattice spacing of the sample, which is fulfilled for optical spectroscopy. On the other hand, Eq. 8.1) is not local in time. Unless there is a clear separation between the time scales that govern the electromagnetic response and the nonequilibrium dynamics of the electronic system, $\sigma\left(t, t^{\prime}\right)$ is no longer time-translationally invariant as it is when the sample is in equilibrium.

Provided that $\sigma\left(t, t^{\prime}\right)$ is known, one can calculate the reflected and transmitted pulses from Maxwell's equations, taking the induced current inside the sample from Eq. (8.1) [225, 226, 227]. However, the solution of those equations, and the coprresponding derivation of generalized reflection and transmission coefficients is more complicated than for samples that are in equilibrium. In fact, even to define those coefficients in a nonequilibrium sitation is more involved than in the equilibrium case. For this purpose consider a typical time-resolved reflection experiment, performed at normal incidence, on a sample that is infinite in the $y-z$ plane (cf. Fig. 8.1). Outside the sample light propagates with constant velocity $c$, and incident and reflected pulses at $x \rightarrow-\infty$ are given by $\boldsymbol{E}_{0}(t, x)$ $=\hat{\boldsymbol{z}} E_{0}\left(t-t_{d}-x / c\right)$ and $\boldsymbol{E}_{\mathrm{refl}}(t, x)=\hat{\boldsymbol{z}} E_{\mathrm{refl}}\left(t-t_{d}+x / c\right)$, respectively. Here we have introduced the wave packets $E_{0}(\tau)$ and $E_{\text {refl }}(\tau)$, which are centered around $\tau=0$, and $t_{d}$ is the probe delay. For simplicity, it was assumed that the polarization direction $\hat{\boldsymbol{z}}$ for both pulses is the same, which holds, e.g., when the sample has cubic symmetry and pump and 


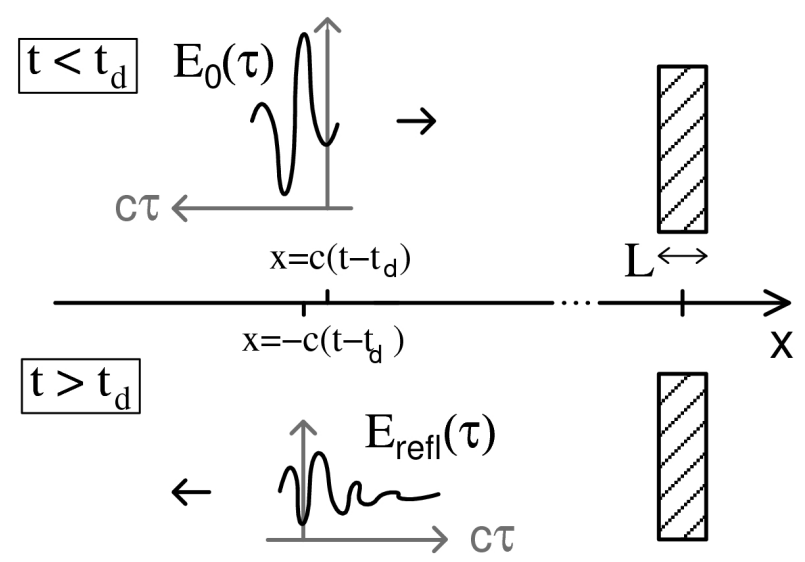

Figure 8.1: (Reprinted from Ref. [72]) Time-resolved reflection experiment. For $t \rightarrow$ $-\infty$ a probe pulse $\boldsymbol{E}_{0}(t, x)=\hat{\boldsymbol{z}} E_{0}\left(t-t_{d}-x / c\right)$ propagates in $+x$ direction without dispersion (upper panel). The sample is hit at times near $t=t_{d}$, and emits the reflected pulse $\boldsymbol{E}_{\text {refl }}(t, x)=\hat{z} E_{\text {refl }}\left(t-t_{d}+x / c\right)$, which propagates in $-x$ direction after leaving the sample (lower panel).

probe are collinear. We can then define a generalized reflection coefficient $r\left(t, t^{\prime}\right)$ [225],

$$
E_{\mathrm{refl}}(\tau)=\int_{0}^{\infty} d s r\left(t_{d}+\tau, t_{d}+\tau-s\right) E_{0}(\tau-s),
$$

which gives a linear relation between the two pulses. Provided that the full time dependent electrical field can be measured (as in time-resolved terahertz spectroscopy [220, 221]), the two-time reflection coefficient $r\left(t, t^{\prime}\right)$ can be deduced from experiment by suitably choosing the probe pulse and measuring at all possible pump-probe delays $t_{d}$. The mathematical structure of Eq. (8.2) has several important consequences. Most importantly, Eq. (8.2) clearly shows that a sample which is not in equilibrium can modulate the pulse frequency, because for a nonequilibrium state $\sigma\left(t, t^{\prime}\right)$ and $r\left(t, t^{\prime}\right)$ depends on $t$ and $t^{\prime}$ separately. Furthermore, light which is reflected or transmitted from a sample which is not in equilibrium can in principle be stronger than the incident light, corresponding to a gain out of the nonequilibrium state [70].

If the optical conductivity $\sigma\left(t, t^{\prime}\right)$ depends on $t$ and $t^{\prime}$ separately, the relation between $r\left(t, t^{\prime}\right)$ and $\sigma\left(t, t^{\prime}\right)$ can be quite involved [227]. An approximate form for $r\left(t, t^{\prime}\right)$, which is valid for reflection from a very thin slab with thickness $L \rightarrow 0$, such that the phase lag between the borders is negligible, is given by [72]

$$
r\left(t, t^{\prime}\right)=\frac{L}{c} \sigma\left(t, t^{\prime}\right)
$$

A more realistic description must takes the finite thickness of the sample and its inhomogeneous excited state into account, and it usuyally requires the numerical simulation of the wave propagation in the medium[226, 227]. 


\subsubsection{Optical Conductivity in DMFT}

The current operator for the Hubbard Hamiltonian (1.1) with Peierls phase factors $(1.2)$ is defined [17, 18] by the relation $\boldsymbol{j}(\boldsymbol{r})=-c \delta H / \delta \boldsymbol{A}(\boldsymbol{r}) 2^{2}$ Using Eq. 1.2, we obtain the current in the long wave-length limit as

$$
\begin{aligned}
\langle\boldsymbol{j}(t)\rangle & =\left\langle\frac{1}{V} \int d^{d} \boldsymbol{r} \boldsymbol{j}(\boldsymbol{r}) e^{i \boldsymbol{q} \boldsymbol{r}}\right\rangle_{\boldsymbol{q} \rightarrow 0}, \\
& =\frac{i e}{V} \sum_{\boldsymbol{k} \sigma} \boldsymbol{v}_{\boldsymbol{k} \sigma}(t) G_{\boldsymbol{k} \sigma}^{<}(t, t),
\end{aligned}
$$

where the current vertex is given by

$$
\boldsymbol{v}_{\boldsymbol{k} \sigma}(t)=\hbar^{-1} \partial_{\boldsymbol{k}} \epsilon_{\boldsymbol{k} \sigma}(t)=\hbar^{-1} \partial_{\boldsymbol{k}} \tilde{\epsilon}_{\boldsymbol{k}+\frac{e}{\hbar c}} \boldsymbol{A}(t), \sigma .
$$

and $V$ is the volume of the sample. Since we are only interested in the linear current response to a weak probe field, we define the susceptibility

$$
\chi_{\alpha \beta}\left(t, t^{\prime}\right)=\delta\left\langle j_{\alpha}(t)\right\rangle / A_{\beta}\left(t^{\prime}\right) .
$$

In the chosen gauge with $\boldsymbol{E}(t)=-\partial_{t} \boldsymbol{A}(t) / c$, the susceptibility $\chi_{\alpha \beta}\left(t, t^{\prime}\right)$ is related to the optical conductivity $\sigma_{\alpha \beta}\left(t, t^{\prime}\right)[$ Eq. (8.1)] by

$$
\sigma_{\alpha \beta}\left(t, t^{\prime}\right)=-c \int_{t^{\prime}}^{\infty} d \bar{t} \chi_{\alpha \beta}(t, \bar{t}) .
$$

The susceptibility (8.5) is related to the current-current correlation function, which can be evaluated in analogy to the equilibrium case [228]. Here we prefer to take the derivative of $8.4 \mathrm{~b}$ directly, where the vector potential enters both in the vertex $\boldsymbol{v}_{\boldsymbol{k} \sigma}(t)$ and in the Green function $G_{\boldsymbol{k} \sigma}^{<}(t, t)$. This yields the diamagnetic and paramagnetic contributions to the susceptibility,

$$
\begin{aligned}
\chi_{\alpha \beta}\left(t, t^{\prime}\right) & =\chi_{\alpha \beta}^{\mathrm{dia}}\left(t, t^{\prime}\right)+\chi_{\alpha \beta}^{\mathrm{pm}}\left(t, t^{\prime}\right), \\
\chi_{\alpha \beta}^{\mathrm{dia}}\left(t, t^{\prime}\right) & =\frac{i e}{V} \sum_{\boldsymbol{k} \sigma} \frac{\delta v_{\boldsymbol{k} \sigma}^{\alpha}(t)}{\delta A_{\beta}\left(t^{\prime}\right)} G_{\boldsymbol{k} \sigma}^{<}(t, t), \\
\chi_{\alpha \beta}^{\mathrm{pm}}\left(t, t^{\prime}\right) & =\frac{i e}{V} \sum_{\boldsymbol{k} \sigma} v_{\boldsymbol{k} \sigma}^{\alpha}(t) \frac{\delta G_{\boldsymbol{k} \sigma}^{<}(t, t)}{\delta A_{\beta}\left(t^{\prime}\right)} .
\end{aligned}
$$

The paramagnetic contribution can be found from a variation of the lattice Dyson equation (3.14),

$$
\delta G_{\boldsymbol{k} \sigma}=-G_{\boldsymbol{k} \sigma} *\left[\delta \mathcal{G}_{\boldsymbol{k} \sigma}^{-1}-\delta \Sigma_{\sigma}\right] * G_{\boldsymbol{k} \sigma} .
$$

\footnotetext{
${ }^{2}$ The current is gauge-invariant and satisfies the continuity equation for the density $\rho(\boldsymbol{r})=\sum_{i \sigma} \delta(\boldsymbol{r}-$ $\left.\boldsymbol{R}_{i}\right) c_{i \sigma}^{\dagger} c_{i \sigma}$, as under a gauge transformation the Hamiltonian transforms as $H\{\boldsymbol{A}+\boldsymbol{\nabla} \Lambda\}=e^{-i g} H\{\boldsymbol{A}\} e^{i g}$, where $g=\frac{e}{\hbar c} \int d^{d} \boldsymbol{r} \Lambda(\boldsymbol{r}) \rho(\boldsymbol{r})$.
} 
Some simplifications occur in the absence of anisotropies. We note that the second term in (8.8), containing the $\boldsymbol{k}$-independent self-energy, does not contribute to the $\boldsymbol{k}$ sum in Eq. (8.7c) if (i) $G_{\boldsymbol{k} \sigma}$ is symmetric under inversion of $\boldsymbol{k}$ and (ii) the vertex $\boldsymbol{v}_{\boldsymbol{k} \sigma}$ is antisymmetric. These conditions are met by an isotropic system without external fields, and are therefore generally valid for systems with inversion symmetry in equilibrium [229]. However, the isotropy may be lost when an initially isotropic system is driven out of equilibrium, e.g., when a current is induced by the electrical pump field. Furthermore, the vertex (8.4c) is no longer antisymmetric when an electrical field is present in addition to the probe field, i.e., when the paramagnetic susceptibility $8.7 \mathrm{c}$ is evaluated at $\boldsymbol{A}$ $\neq 0$. Experimentally these anisotropic effects in otherwise isotropic systems show up as a dependence of the signal on the relative polarization of pump and probe pulses. In order to study such anisotropic effects, vertex corrections contained in $\delta \Sigma_{\sigma}$ must be taken into account (even for cubic lattices), by solving a Bethe-Salpeter equation on the Keldysh contour, with the irreducible vertex function $\delta \Sigma_{\sigma} / \delta G_{\sigma}$ from the auxiliary singlesite problem as input. Recently, these vertex corrections were shown to cause important corrections in a setup where pump and probe are continuous waves, i.e., they overlap [70].

In the following we only consider the completely isotropic relaxation between homogeneous phases, such that the vertex corrections $\delta \Sigma_{\sigma}$ can be disregarded. Eq. (8.7c) is evaluated at zero field, so that only the first term $\delta F_{\boldsymbol{k} \sigma}\left(t_{1}, t_{2}\right)=-\left[G_{\boldsymbol{k} \sigma} * \delta\left(\mathcal{G}_{\boldsymbol{k} \sigma}^{-1}\right) * G_{\boldsymbol{k} \sigma}\right]\left(t_{1}, t_{2}\right)$ contributes to $\delta G_{\boldsymbol{k} \sigma}$ in Eq. (8.8). This corresponds to keeping only the elementary bubble diagram for the current-current correlation function [228]. The two convolutions in $\delta F_{\boldsymbol{k} \sigma}\left(t_{1}, t_{2}\right)$ collapse to a single one because $\left[\delta \mathcal{G}_{\boldsymbol{k} \sigma}^{-1}\right]\left(t, t^{\prime}\right) \propto \delta_{\mathcal{C}}\left(t, t^{\prime}\right)$. In order to obtain $\delta G_{\boldsymbol{k} \sigma}^{<}(t, t)$ we take $t_{1}=t$ and $t_{2}=t$ on the upper and lower branch of the contour, respectively [cf. Table 2.2. The contour integral is then transformed into an integral along the real axis,

$$
\delta F_{\boldsymbol{k} \sigma}\left(t_{+}, t_{-}\right)=\frac{e}{\hbar c} \int_{-\infty}^{\infty} d \bar{t} \boldsymbol{v}_{\boldsymbol{k} \sigma}(\bar{t}) \delta \boldsymbol{A}(\bar{t})\left[G_{\boldsymbol{k} \sigma}^{++}(t, \bar{t}) G_{\boldsymbol{k} \sigma}^{+-}(\bar{t}, t)-G_{\boldsymbol{k} \sigma}^{+-}(t, \bar{t}) G_{\boldsymbol{k} \sigma}^{--}(\bar{t}, t)\right],
$$

from which the optical conductivity $\sigma_{\alpha \beta}\left(t, t^{\prime}\right)$ can be read off. From the relations given in table 2.2 and the general symmetry 2.15 , we finally obtain the paramagnetic susceptibility

$$
\chi_{\alpha \beta}^{\mathrm{pm}}\left(t, t^{\prime}\right)=-2 \chi_{0} \sum_{\boldsymbol{k} \sigma} \tilde{v}_{\boldsymbol{k} \sigma}^{\alpha} \tilde{v}_{\boldsymbol{k} \sigma}^{\beta} \operatorname{Im}\left[G_{\boldsymbol{k} \sigma}^{\mathrm{r}}\left(t, t^{\prime}\right) G_{\boldsymbol{k} \sigma}^{<}\left(t^{\prime}, t\right)\right]
$$

where $\chi_{0}=e^{2} /(V \hbar c)$ and $\tilde{\boldsymbol{v}}_{\boldsymbol{k} \sigma}=\partial_{\boldsymbol{k}} \tilde{\epsilon}_{\boldsymbol{k}, \sigma} / \hbar$. The diamagnetic contribution follows directly from Eqs. 8.4c) and (8.7b):

$$
\chi_{\alpha \beta}^{\operatorname{dia}}\left(t, t^{\prime}\right)=\frac{i \chi_{0}}{\hbar} \delta\left(t-t^{\prime}\right) \sum_{\boldsymbol{k} \sigma}\left(\partial_{\boldsymbol{k}_{\alpha}} \partial_{\boldsymbol{k}_{\beta}} \tilde{\epsilon}_{\boldsymbol{k} \sigma}\right) G_{\boldsymbol{k} \sigma}^{<}(t, t) .
$$

Eqs. 8.6 and 8.10 constitute our final DMFT expressions for the optical conductivity (provided that anisotropic effects are disregarded, as discussed above). 
The optical conductivity (8.6) can be written as

$$
\sigma_{\alpha \beta}\left(t, t^{\prime}\right)=\left[\sigma_{\alpha \beta}^{\mathrm{reg}}\left(t, t^{\prime}\right)+D_{\alpha \beta}(t)\right] \Theta\left(t-t^{\prime}\right)
$$

i.e., it splits into its regular part

$$
\sigma_{\alpha \beta}^{\mathrm{reg}}\left(t, t^{\prime}\right)=c \int_{-\infty}^{t^{\prime}} d \bar{t} \chi_{\alpha \beta}^{\mathrm{pm}}(t, \bar{t})
$$

which vanishes in the limit $t^{\prime} \rightarrow-\infty$, and the Drude contribution

$$
\begin{aligned}
D_{\alpha \beta}(t) & \equiv \lim _{t^{\prime} \rightarrow-\infty} \sigma_{\alpha \beta}\left(t, t^{\prime}\right) \\
& =\sigma_{\alpha \beta}^{\operatorname{dia}}(t)-c \int_{-\infty}^{t} d \bar{t} \chi_{\alpha \beta}^{\mathrm{pm}}(t, \bar{t}),
\end{aligned}
$$

which does not depend on the time difference at all. In the latter expression, $\sigma_{\alpha \beta}^{\text {dia }}(t)=$ $-c \int_{-\infty}^{\infty} d t^{\prime} \chi_{\alpha \beta}^{\mathrm{dia}}\left(t, t^{\prime}\right)$ is the weight of the delta function in Eq. $8.10 \mathrm{~b}$. A finite Drude contribution $D_{\alpha \beta}(t) \neq 0$ indicates perfectly metallic behavior, because it gives rise to a delta function at zero frequency in the partially Fourier-transformed optical conductivity

$$
\begin{aligned}
\tilde{\sigma}_{\alpha \beta}(t, \omega) & =\int_{0}^{\infty} d s e^{i(\omega+i 0) s} \sigma_{\alpha \beta}(t, t-s) \\
& =\tilde{\sigma}_{\alpha \beta}^{\mathrm{reg}}(t, \omega)+\frac{i D_{\alpha \beta}(t)}{\omega+i 0} .
\end{aligned}
$$

Note that Eqs. 8.10) and (8.12) can be checked by inserting equilibrium Green functions [Eq. (2.18c)]

$$
\begin{aligned}
& G_{\boldsymbol{k} \sigma}^{\mathrm{r}}\left(t, t^{\prime}\right)=-i \Theta\left(t-t^{\prime}\right) \int d \omega A_{\boldsymbol{k} \sigma}(\omega) e^{i \omega\left(t^{\prime}-t\right)}, \\
& G_{\boldsymbol{k} \sigma}^{<}\left(t, t^{\prime}\right)=i \int d \omega A_{\boldsymbol{k} \sigma}(\omega) f(\omega) e^{i \omega\left(t^{\prime}-t\right)},
\end{aligned}
$$

into Eq. (8.14), with the spectral function $A_{\boldsymbol{k} \sigma}(\omega)=-\operatorname{Im}\left[G_{\boldsymbol{k} \sigma}^{\mathrm{r}}(\omega+i 0)\right] / \pi$ and the Fermi function $f(\omega)=1 /\left(1+e^{\beta \omega}\right)$. Then the well-known expression for the regular part of the optical conductivity in equilibrium [228],

$$
\operatorname{Re} \sigma_{\alpha \beta}^{\mathrm{reg}}(\omega)=\pi c \chi_{0} \sum_{\boldsymbol{k} \sigma} \tilde{v}_{\boldsymbol{k} \sigma}^{\alpha} \tilde{v}_{\boldsymbol{k} \sigma}^{\beta} \int_{-\infty}^{\infty} d \omega^{\prime} \frac{A_{\boldsymbol{k} \sigma}\left(\omega^{\prime}\right) A_{\boldsymbol{k} \sigma}\left(\omega+\omega^{\prime}\right)\left[f\left(\omega^{\prime}\right)-f\left(\omega+\omega^{\prime}\right)\right]}{\omega},
$$

is recovered. 


\subsubsection{The sum rule for the two-time optical conductivity}

In equilibrium physics, the sum-rule for the optical conductivity is an important concept. The discussion above allows to derive a sum-rule for the two-time optical conductivity. Because $\sigma\left(t, t^{\prime}\right)$ is real, the partial Fourier transform 8.14a satisfies the sum rule

$$
\int_{0}^{\infty} d \omega \operatorname{Re} \tilde{\sigma}_{\alpha \beta}(t, \omega)=\sigma_{\alpha \beta}(t, t) .
$$

The equal-time conductivity $\sigma_{\alpha \beta}(t, t)$ on the right-hand side of this expression can now be obtained directly from Eq. (8.6) in the following way: At $t=t^{\prime}$, only the diamagnetic component $\chi_{\alpha \beta}^{\mathrm{dia}}\left(t, t^{\prime}\right)$ corresponds to the integral 8.6) under two rather general assumptions. These assumptions are (i), that the paramagnetic response is causal, such that $\chi_{\alpha \beta}^{\mathrm{pm}}\left(t, t^{\prime}\right)=0$ for $t<t^{\prime}$, and (ii), that there is no instantaneous singular respone of the Green function to an external vector potential, i.e., $\chi_{\alpha \beta}^{\mathrm{pm}}\left(t, t^{\prime}\right)$ is finite at $t=t^{\prime}$. For the case that vertex corrections do not contribute to $\chi_{\alpha \beta}^{\mathrm{pm}}\left(t, t^{\prime}\right)$, both assumptions follow from Eq. 8.10a because $G_{\boldsymbol{k} \sigma}^{\mathrm{r}}(t, t)$ and $G_{\boldsymbol{k} \sigma}^{<}(t, t)$ are purely imaginary. More generally, assumption (ii) implies that the Fourier transform of $\chi_{\alpha \beta}^{\mathrm{pm}}(t, t-s)$ vanishes in the high frequency limit. Under the assumptions (i) and (ii) we obtain

$$
\lim _{s \downarrow 0} \sigma_{\alpha \beta}(t, t-s)=-c \int_{t-s}^{\infty} d \bar{t} \chi_{\alpha \beta}^{\operatorname{dia}}(t, \bar{t}),
$$

which together with Eqs. (8.10b) and (8.17) yields the general sum rule for the nonequilibrium optical conductivity,

$$
\int_{0}^{\infty} d \omega \operatorname{Re} \tilde{\sigma}_{\alpha \beta}(t, \omega)=\frac{e^{2}}{\hbar^{2} V} \sum_{\boldsymbol{k} \sigma}\left(\partial_{\boldsymbol{k}_{\alpha}} \partial_{\boldsymbol{k}_{\beta}} \epsilon_{\boldsymbol{k} \sigma}\right) n_{\boldsymbol{k} \sigma}(t) .
$$

Here we have also used that $-i G_{\boldsymbol{k} \sigma}^{<}(t, t)=n_{\boldsymbol{k} \sigma}(t)$.

\subsection{Photoemission spectroscopy in the sudden approxi- mation}

\subsubsection{Introduction}

A theoretical decsription of time-resolved photoemission spectroscopy (TRPES) should both take into account that the electrons are not in equilibrium, and that the signal can depend on the pulse shape of the probe pulse, in particular the pulse duration. Recently, Freericks, Krishnamurthy, and Pruschke [230] extened existing theories of conventional photoemission spectroscopy to the case where the sample is not in equilibrium and measurement pulses have a finite time duration. Their approach relates the photoemission intensity as a function of the probe pulse delay time to electronic one-particle real-time 
Green functions of the sample, which fully incorporate the excitation through the pump pulse. In contrast, earlier Green function approaches have treated both pump and probe process on the same perturbative level [231].

The relation to real-time Green functions allows us to make direct contact to nonequilibrium DMFT, and study how details of the electronic thermalization process after the pump excitation become manifest in time-resolved photoemission experiments. In this context it is crucial to take into account that a probe pulse of finite duration $\Delta t$ has a finite width $\Delta \omega$ in frequency space, such that $\Delta t$ and $\Delta \omega$ satisfy the frequency-time uncertainty $\Delta t \Delta \omega \geq 2 \pi$. As we will see below, the relaxation of the electronic system can be so fast that pulses which can resolve the time evolution of the quantum state have a very poor energy resolution, such that not much more than the total weight of the electronic spectrum is recorded. As a consequence, it can be practically impossible to obtain the nonequilibrium electronic Green functions from the photoemission spectrum in some cases [73], although the converse procedure is straightforward within the theoretical description of TRPES given in Ref. [230].

\subsubsection{General derivation}

Photoemission experiments with both temporal and angular resolution probe the sample with a pulse of finite duration. Although a short pulse has necessarily finite extent in space, its spread in wavenumber can nevertheless be neglected due to the steep dispersion of light, and we can thus assume that the pulse has a definite wave number $\boldsymbol{q}$. In fact, even when the frequency width $\Delta \omega$ of the pulse is on the order of a typical bandwidth $(\hbar \delta \omega=1 \mathrm{eV})$, the spread in wavenumber, $\delta q=|\delta \omega / c| \approx 0.0005 / \AA$, is small compared to the typical extent of the Brillouin zone. The detector is sensitive to the kinetic energy $E$ $=\hbar^{2} k_{e}^{2} / 2 m$ of the photoelectrons and to the direction $\hat{\boldsymbol{k}}_{e}$ in which they are emitted, but not to their arrival time $\left(\boldsymbol{k}_{e}=\hat{\boldsymbol{k}}_{e} k_{e}\right.$ is the photoelectron momentum).

As in Ref. [230] we define the time-resolved photoemission signal by the total number of electrons that are emitted per solid angle $d \Omega_{\hat{k}_{e}}$ and energy interval $d E$

$$
I\left(\hat{\boldsymbol{k}}_{e}, E ; \boldsymbol{q}, t_{p}\right)=\frac{d N\left(\hat{\boldsymbol{k}}_{e}, E ; \boldsymbol{q}, t_{p}\right)}{d \Omega_{\hat{\boldsymbol{k}}_{e}} d E}
$$

in response of a probe pulse at time $t_{p}$ [230], and do not include direct photoemission due to the pump pulse. Freericks et al. have derived an expression for the photoemission signal (8.20) under certain approximations [230], which we shall describe in the following. For this purpose one needs to include both the electronic states in the solid and the outgoing electron states in the Hamiltonian, which thus takes the following form

$$
\begin{aligned}
& H=H_{\text {solid }}(t)+H_{\text {free }}+H_{\text {coupling }}(t) \\
& H_{\text {free }}=\sum_{\boldsymbol{k}_{e} \sigma}(E+\Phi) a_{\boldsymbol{k}_{e} \sigma}^{\dagger} a_{\boldsymbol{k}_{e} \sigma} \\
& H_{\text {coup }}(t)=S\left(t-t_{p}\right) \sum_{\boldsymbol{k} \boldsymbol{k}_{e} \sigma} M\left(\boldsymbol{k}, \boldsymbol{q} ; \boldsymbol{k}_{e}\right) e^{i c q t} a_{\boldsymbol{k}_{e} \sigma}^{\dagger} c_{\boldsymbol{k} \sigma}+\text { h.c. }
\end{aligned}
$$


In this expression, the system Hamiltonian $H_{\text {solid }}(t)$ contains only the degrees of free$\operatorname{dom} c_{\boldsymbol{k}_{e}}^{(\dagger)}$ in the solid, e.g., the valence electrons. Below $H_{\text {solid }}(t)$ will be a simple model Hamiltonian, such as the Hubbard model or the Falicov-Kimball model, which may be time-dependent due to external pump fields. The second part $H_{\text {free }}$ describes electrons in the outgoing states, which are scattering states with asymptotic momentum $\boldsymbol{k}_{e}$. The energy of those vacuum states is shifted with respect to the solid by the work function $\Phi$. The third term $H_{\text {coup }}(t)$ describes the coupling between Bloch states with quasimomentum $\boldsymbol{k}$ in the solid and one-electron scattering states with asymptotic momentum $\boldsymbol{k}_{e}$ via absorption of a photon with momentum $\boldsymbol{q}$. The transition is characterized by the matrix element $M\left(\boldsymbol{k}, \boldsymbol{q} ; \boldsymbol{k}_{e}\right)$, which are derived from the usual $\boldsymbol{A} \cdot \boldsymbol{p}$ coupling of electromagnetic fields in the Hamiltonian. The light field has frequency $e^{i c q t}$ and its amplitude is proportional to the (real) pulse envelope function $S(\tau)$. The latter is defined in a way that it is centered at $\tau=0$, such that the coupling in 8.21 occurs around time $t=t_{p}$.

In writing down the Hamiltonian (8.21) i has been assumed that there are no interaction terms between the outgoing electrons and the electrons in the solid, which is equivalent to the so-called sudden approximation [232]. Without this assumption the photocurrent is related to complicated three-current correlation functions [233]. Similar to the derivation of the optical response in Sec 8.2 ome can now assume that the probe field is weak, and compute the total number of electrons emitted during one probe pulse in convential time-dependent perturbattion theory. The photoemission signal 8.20 is then only related to matrix elements $M\left(\boldsymbol{k}, \boldsymbol{q} ; \boldsymbol{k}_{e}\right)$ and the real-time one-particle Green function [230]

$$
G_{\boldsymbol{k}, \boldsymbol{k}^{\prime}}^{<}\left(t, t^{\prime}\right)=i \operatorname{Tr}\left[\rho_{0} \hat{c}_{\boldsymbol{k}^{\prime} \sigma}^{\dagger}\left(t^{\prime}\right) \hat{c}_{\boldsymbol{k} \sigma}(t)\right],
$$

which incorporates the full nonequilibrium dynamics of the sample, including all external fields except for the probe.

The presence of the surface and the dependence of photoemission spectra on matrix elements can substantially complicate the comparison of theoretical and experimental data for specific materials. In order to reveal general aspects of TRPES one often has to resort to further approximations. in the following we assume that photoemission measures the bulk properties of the sample which are contained in the momentum-diagonal Green function $G_{\boldsymbol{k}}^{<}\left(t, t^{\prime}\right) \equiv G_{\boldsymbol{k} \boldsymbol{k}}^{<}\left(t, t^{\prime}\right)$ of the infinite and translationally invariant system, and (ii), we take matrix elements to be constant, apart from the constraint that momentum must be conserved in the plane of the surface, i.e., $M\left(\boldsymbol{q}, \boldsymbol{k} ; \boldsymbol{k}_{e}\right) \equiv M \delta_{\boldsymbol{k}_{\|}+\boldsymbol{q}_{\|}, \boldsymbol{k}_{e \|}}$. The timeresolved photoemission spectrum 8.20 is then given by [230]

$$
\begin{aligned}
I\left(\hat{\boldsymbol{k}}_{e}, E ; \boldsymbol{q}, t_{p}\right) & \propto \sum_{\boldsymbol{k} \sigma} \delta_{\boldsymbol{k}_{\|}+\boldsymbol{q}_{\|}, \boldsymbol{k}_{e \|}} \mathcal{I}_{\boldsymbol{k} \sigma}\left(E-c q-\Phi ; t_{p}\right), \\
\mathcal{I}_{\boldsymbol{k} \sigma}\left(\omega ; t_{p}\right) & =-i \int d t \int d t^{\prime} S(t) S\left(t^{\prime}\right) e^{i \omega\left(t^{\prime}-t\right)} G_{\boldsymbol{k} \sigma}^{<}\left(t+t_{p}, t^{\prime}+t_{p}\right) .
\end{aligned}
$$

In the following we will simply refer to Eq. (8.24) as the photoemission intensity. The rather general aspects of the momentum- and frequency-dependent expression (8.24) that are discussed below presumably persist after summation over some part of the Brillouin zone [Eq. (8.23)]. 
When the system is in equilibrium, Eq. (8.24) can be reduced to a form that is directly related to the well-known expression

$$
\mathcal{I}_{\boldsymbol{k} \sigma}^{e q .}(\omega) \propto A_{\boldsymbol{k} \sigma}(\omega) f(\omega)
$$

for the intrinsic photocurrent in continuous beam experiments [234, 235]. in terms of the equilibrium spectral function $A_{\boldsymbol{k} \sigma}(\omega)$ and the Fermi function $f(\omega)$. In equilibrium situations, Green functions depend on the time difference only, and one can use the Fourier transform [cf. Eq. 2.18c

$$
\int d t e^{i \omega t} G_{\boldsymbol{k} \sigma}^{<}(t, 0)=2 \pi i A_{\boldsymbol{k} \sigma}(\omega) f(\omega)
$$

to simpify Eq. 8.24). The photoemission intensity then reduces to

$$
\mathcal{I}_{\boldsymbol{k} \sigma}(\omega)=\int d \omega^{\prime}\left|\tilde{S}\left(\omega+\omega^{\prime}\right)\right|^{2} A_{\boldsymbol{k} \sigma}\left(\omega^{\prime}\right) f\left(\omega^{\prime}\right)
$$

which is a convolution of the continuous beam expression with the Fourier-transform $\tilde{S}(\omega)$ $=\int d t S(t) e^{i \omega t}$ of the pulse envelope.

\subsubsection{Role of the energy-time uncertainty relation}

The continuous beam photoemission intensity (8.25) has a simple interpretation in terms of the occupied states in the solid. By contrast, an analogous interpretation of the pumpprobe intensity (8.24) is not always possible due to the frequency-time uncertainty of the probe pulse: The energy $\epsilon$ of occupied states in the solid from which photoelectrons are released is determined from the kinetic energy of the photoelectrons, the work function of the solid, and the photon energy $E_{\gamma}=\hbar \omega$. Using measurement pulses of finite duration $\Delta t$, the latter can be determined only up to an uncertainty $\delta E_{\gamma} \gtrsim \hbar / \Delta t$. In a strongly correlated electron system we expect that typical relaxation times are directly related to the energy scales that appear in the spectrum, such that all information on the initial energy $\epsilon$ is lost for pulses which are short enough to resolve the electronic dynamics.

The frequency-time uncertainty is already evident in the signal (8.27) for the measurement of equilibrium states with pulses of finite duration. In Eq. (8.27) the frequencydependent spectrum is broadened with a positive function $|\tilde{S}(\omega)|^{2}$ that has a width of the order of $\Delta \omega>1 / \Delta t$. This broadening of equilibrium spectra has been called the "windowing effect" by the authors of Ref. [230]. It can be important for experiments, which can be discussed within some kind of quasiequilibrium approximation, i.e, electrons can be assumed to be in equilibrium at any time, but their state changes due to parameters such as an electronic temperature (see, e.g., Ref. [204]).

By contrast, in this section we consider systems with a purely Hamiltonian time evolution involving only electronic degrees of freedom, and investigate TRPES with ultrashort pulses that do resolve the thermalization of the electrons after the pump pulse. The electronic state is then characterized by a real-time Green functions $G_{\boldsymbol{k} \sigma}^{<}(t+s, t)$ depending on two time variables, which contains important information both as a function of the 
absolute time $t$ and of the time difference $s$ between addition and removal of an electron (see below). We will demostrate from Eq. (8.24) that in this case the full time dependence on both time variables cannot be recovered from the time-dependent photoemission intensity, no matter how the pulse length of the probe pulse is chosen [73].

To make this explicit it is helpful to express the Green function in Eq. 8.24 through a partial Fourier transform,

$$
\widetilde{G}(\omega, t)=\int d s e^{i \omega s} G(t+s, t)
$$

which yields

$$
\mathcal{I}_{\boldsymbol{k} \sigma}\left(\omega ; t_{p}\right)=-i \int d t \int d \omega^{\prime} S(t) \widetilde{S}\left(\omega^{\prime}\right) e^{-i \omega^{\prime} t} \widetilde{G}_{\boldsymbol{k} \sigma}^{<}\left(\omega+\omega^{\prime}, t+t_{p}\right) .
$$

This expression shows that the photoemission signal corresponds to a simultaneous convolution of the partially Fourier transformed Green function with the pulse shapes $S(t)$ in time and its Fourier transform $\widetilde{S}(\omega)$, such that the accuracies $\Delta \omega$ and $\Delta t$ in frequency and time with which $\widetilde{G}^{<}(\omega, t)$ is measured cannot be independently small due to the uncertainty relation $\Delta \omega \Delta t \geq 2 \pi$.

The energy-time uncertainty can also be accessed by the real-time Green function $G_{\boldsymbol{k} \sigma}^{<}(t, t+s)$ directly. When the probe pulse extends only over a finite duration $\Delta t$, the product $S(t) S\left(t^{\prime}\right)$ in Eq. (8.24) vanishes for all $t-t^{\prime}>\Delta t$, and hence Eq. (8.24) is independent of the values of $G_{\boldsymbol{k} \sigma}^{<}(t, t+s)$ at $s>\Delta t$. In other words, $G_{\boldsymbol{k} \sigma}^{<}(t, t+s)$ at fixed $s$ cannot be measured with a time resolution (in $t$ ) which is better that $s$. This is also true for pulses which do not have a strictly finite duration in time, e.g., Gaussian pulses

$$
S(t)=\exp \left(-\frac{t^{2}}{2 \Delta t^{2}}\right)
$$

When we attempt to invert the convolution of $G_{\boldsymbol{k} \sigma}^{<}\left(t, t^{\prime}\right)$ in Eq. 8.24) via a Fourier transform

$$
\widetilde{\mathcal{I}}_{\boldsymbol{k} \sigma}\left(s ; t_{p}\right)=\int d \omega e^{i \omega s} \mathcal{I}_{\boldsymbol{k} \sigma}\left(\omega ; t_{p}\right)
$$

we obtain

$$
\widetilde{\mathcal{I}}_{\boldsymbol{k} \sigma}\left(s ; t_{p}\right) \propto \exp \left(-\frac{s^{2}}{4 \Delta t^{2}}\right) \int d t G_{\boldsymbol{k} \sigma}^{<}\left(t_{p}+\frac{s}{2}+t, t_{p}-\frac{s}{2}+t\right) \exp \left(-\frac{t^{2}}{\Delta t^{2}}\right) .
$$

While the integral in 8.32 apparently measures $G_{\boldsymbol{k} \sigma}^{<}\left(t_{p}+s / 2, t_{p}-s / 2\right)$ with a time resolution of $\Delta t$, it is practically impossible to choose $\Delta t \ll s$ because then the result vanishes compared to any noise added to $\widetilde{\mathcal{I}}_{\boldsymbol{k} \sigma}\left(s ; t_{p}\right)$, due to the Gaussian prefactor. The form of this prefactor is due to the specific pulse shape 8.30 , but the suppression of the signal for $\Delta t \ll s$ is independent of the pulse shape.

We conclude that at least sophisticated deconvolution techniques are needed to compute the nonequilibrium two-time Green function from the time-resolved photoemission 
result. This will be illustrated in the following section for nonequilibrium situations in the Falicov-Kimball model and the Hubbard model. On the other hand, the converse procedure of calculating the spectrum from the Green function is straightforward. For example, we will show in Sec. 8.4.2 that characteristic signatures of the dynamics of the excited Mott insulator can be revealed from a variation of the pulse length.

\subsection{Evaluation within DMFT}

In the following section we illustrate the general results for time-resolved spectroscopy of the previous two sections within idealized experiments in which the system is suddenly driven out of a metallic or insulating equilibrium state, and subsequently relaxes to a new phase. The Hamiltonian is either given by the Falicov-Kimball model or by the Hubbard model. The nonequilibrium Green functions that enter Eq. (8.10) for the time-resolved optical conductivity, and Eq. (8.24) for TRPES, are obtained with nonequilibrium DMFT as explained in Ch. 5 and Ch. 6 for the Falicov-Kimball model and the Hubbard model, respectively: $]^{3}$

We focus on two different physical situations, namely the buildup of a correlated metal from an uncorrelated state, where quasiparticles form due to the interaction (Sec. 8.4.1), and the relaxation of an excited Mott insulator, where characteristic collapse-and-revival become visible both in the photoemission spectrum and in time-resolved optical spectroscopy (Sec. 8.4.2).

\footnotetext{
$\epsilon$,

$$
\begin{aligned}
\frac{1}{N} \sum_{\boldsymbol{k}} \tilde{v}_{\boldsymbol{k} \sigma}^{\alpha} \tilde{v}_{\boldsymbol{k} \sigma}^{\beta} g\left(\tilde{\epsilon}_{\boldsymbol{k} \sigma}\right) & =\int_{-\infty}^{\infty} d \epsilon D_{\alpha \beta}^{\sigma}(\epsilon) g(\epsilon) \\
\frac{1}{\hbar^{2} N} \sum_{\boldsymbol{k}}\left(\partial_{\boldsymbol{k}_{\alpha}} \partial_{\boldsymbol{k}_{\beta}} \tilde{\epsilon}_{\boldsymbol{k} \sigma}\right) g\left(\tilde{\epsilon}_{\boldsymbol{k} \sigma}\right) & =\int_{-\infty}^{\infty} d \epsilon\left[\partial_{\epsilon} D_{\alpha \beta}^{\sigma}(\epsilon)\right] g(\epsilon),
\end{aligned}
$$
}

${ }^{3}$ In Ch. 5 and 6 we have assumed a semielliptic density of states with quarter bandwidth $V$, which is obtained for nearest-neighbor hopping on the Bethe lattice, but also for a particular choice of longer range hopping amplitudes on the hypercubic lattice in infinite dimensions [180]. In contrast to the results of Ch. 5 and 6 , which depend only on the density of states, the optical conductivity depends explicitely on the dispersion $\epsilon_{\boldsymbol{k} \sigma}$ through the current vertices and their $\boldsymbol{k}$-derivatives in Eq. (8.10). In the following we present results that correspond to hopping on a hypercubic lattice with a semielliptic density of states. In this case one must replace the momentum summations in Eq. 8.10 by integrations over the band-energy

where $N$ is the number of $\boldsymbol{k}$-points, $g(\epsilon)$ is an arbitrary function of the band-energy $\epsilon$, and the dispersionrelated function $D(\epsilon)$ is given by 180 .

$$
D_{\alpha \beta}^{\sigma}(\epsilon)=\delta_{\alpha \beta} \frac{2 V a^{2}}{4 \hbar^{2} \sqrt{1-(\epsilon / 2 V)^{2}}} \exp \left[-2 \operatorname{erf}^{-1}\left(\frac{\epsilon \sqrt{1-(\epsilon / 2 V)^{2}}+W \sin ^{-1}(\epsilon / 2 V)}{\pi V}\right)^{2}\right]
$$

and $a$ is the lattice constant. For the Falicov-Kimball model, one has $D_{\alpha \beta}^{\uparrow}=0$ for the immobile species. 

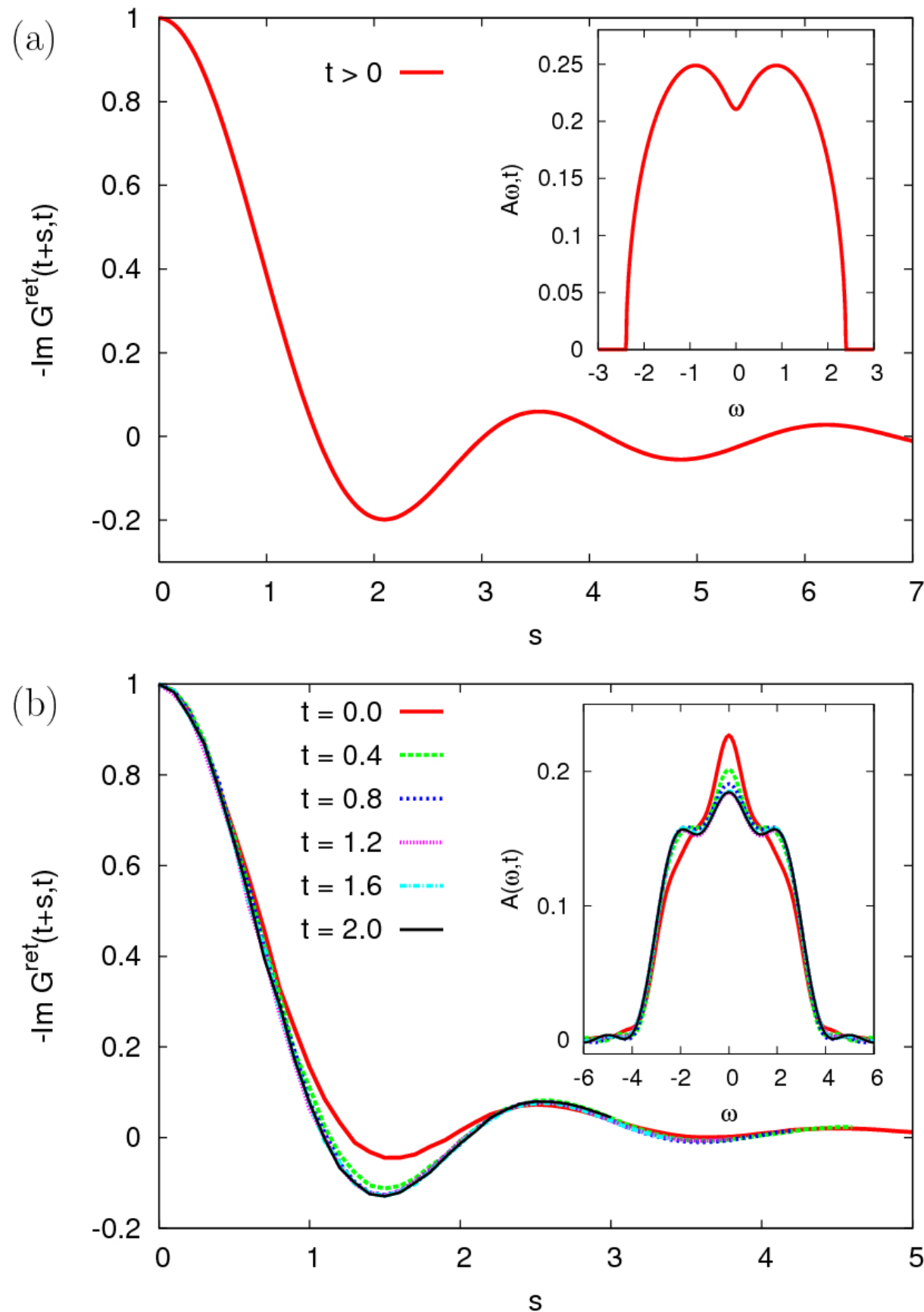

Figure 8.2: Local retarded Green function $G^{\mathrm{r}}(t+s, t)$ and spectrum 8.36) (inset) after a quench from the noninteracting state to the metallic parameter regime in the FalicovKimball model [(a), $U=1.5]$ and the Hubbard model [(b), $U=3]$. 


\subsubsection{Buildup of correlations in the metallic phase}

Following the discussion at the end of Sec. 8.1, the formation of a correlated metallic state from an uncorrelated electron gas can be modelled by an interaction quench from the noninteracting state to finite interaction $U$. Below we compare results for the Hubbard model and the Falicov-Kimball model, that were obtained from nonequilibrium DMFT, using a semielliptic density of states with quarter bandwidth $V$ (Ch. 5 and 6). The energy scale is set by $V=1$, and time is measured in terms of the inverse hopping $\hbar / V$.

The formation of quasiparticles with time can be seen most directly from the momentum resolved real-time Green function $G_{\boldsymbol{k}}(t+s, t)=-i\left\langle\mathrm{~T}_{\mathcal{C}} \hat{c}_{\boldsymbol{k}}(t+s) \hat{c}_{\boldsymbol{k}}^{\dagger}(t)\right\rangle_{0}$, which shows how single-particle excitations that are created in the system at time $t$ decay until later time $t+s$. Important differences between the Falicov-Kimball model and the Hubbard model are already evident from the retarded component of this Green function after integration over momenta [the local Green function $G^{\mathrm{r}}(t+s, t)$ ], and the spectrum

$$
A(\omega, t)=-\frac{1}{\pi} \operatorname{Im} \int_{0}^{\infty} d s e^{i \omega s} G^{\mathrm{r}}(t+s, t),
$$

which is plotted in Fig. 8.2. Note that for this definition the spectrum differs from the noninteracting spectrum of the initial equilibrium state [semielliptic density of states, Eq. (3.18)] at $t=0$ because the Fourier transformation in Eq. (8.36) is only over the interval $s>0$ by definition. For both Hubbard model and Falicov-Kimball model, Eq. (8.36) is given by the interacting equilibrium spectrum for large times after the quench. For the Hubbard model (Fig. $8.2 \mathrm{~b}$ ) there is an indication of the well-known three peak structure with a quasiparticle peak and Hubbard bands [11], while for the Falicov-Kimball model the quasiparticle peak is missing because the metallic state is not a Fermi liquid [171]. The most profound difference between the two cases exists in the dynamics, i.e., the dependence on time $t$ : While in the Hubbard model the final spectrum is reached only after a finite relaxation time $(t \gtrsim 0.6)$ 出 the retarded function of the Falicov-Kimball model is completely independent on time for all times $t>0$. The reason for this is that the decay of $G^{\mathrm{r}}(t+s, t)$ with time-difference $s$ is due to scattering on fixed particles in the Falicov-Kimball model, while in the Hubbard model the scattering particles itself have a nontrivial time-dependence.

Can TRPES distinguish the rapid relaxation of the spectrum 8.36 in the Hubbard model from an instantaneous change such as for the Falicov-Kimball model? To discriminate between those two different physical situations, one has to measure the Green function $G^{\mathrm{r}}(t+s, t)$ with a time-resolution better than the relaxation time. However, from Fig. $8.2 \mathrm{~b}$ one can see that the $t$-dependence of $G^{\mathrm{r}}(t+s, t)$ for the Hubbard model is most pronounced around $s \approx 1.5$, and the final value is almost reached at time $t=$ 0.3. In view of the discussion below Eq. 8.32, however, it is not possible to measure $G^{\mathrm{r}}(t+s, t)$ from TRPES with time resolution considerable better than $\Delta t=s$, due to

\footnotetext{
${ }^{4}$ We have chosen $U=3$ for the Hubbard model, which is in the regime of rapid thermalization discussed in Ch. 6 .
} 


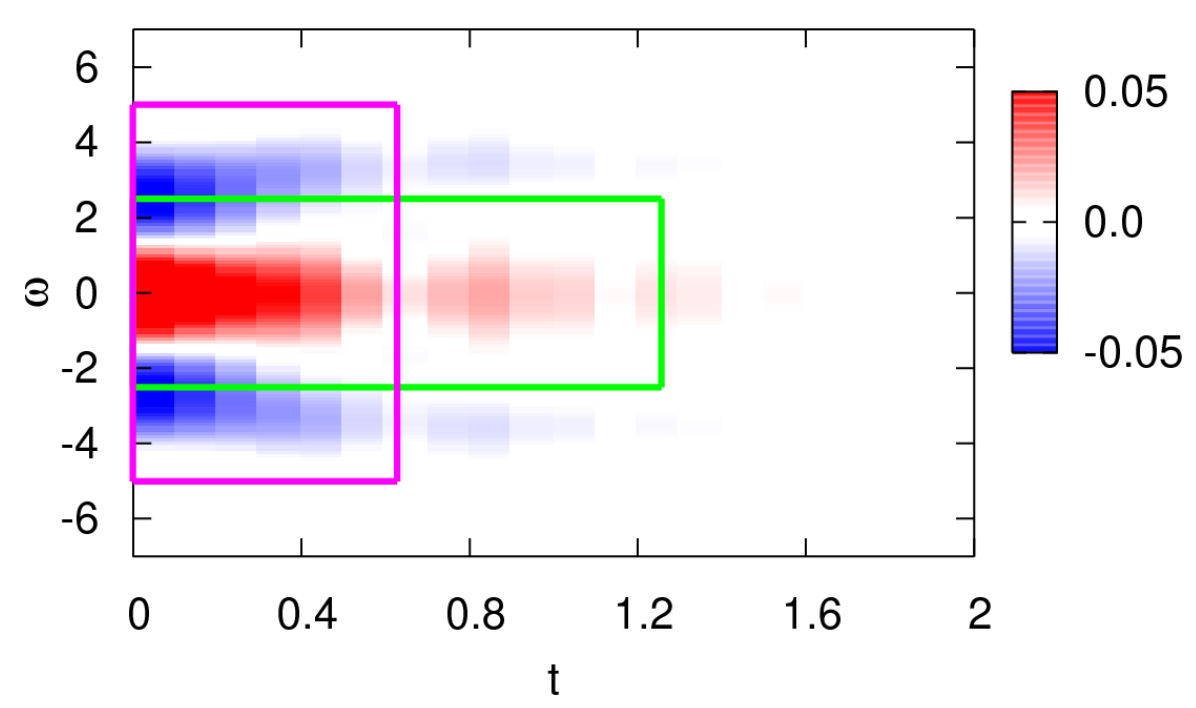

Figure 8.3: Difference $A(\omega, t)-A(\omega, t=2)$ between the spectrum 8.36) at time $t$ and after relaxation, for a quench in the Hubbard model from the noninteracting ground state to interaction $U=3$ (see also Fig. 8.2). The rectangles have an area $\Delta \omega \Delta t=2 \pi$, corresponding to the minimal simultaneous uncertainty of freqency and time whith which the Green function $\tilde{G}^{<}(\omega, t)$ can be obtained from TRPES.

the energy-time uncertainty $\left.\right|^{5}$

The implication of the energy-time uncertainty can be visualized in yet another way. In a plot of the difference of the spectrum 8.36) to its value after relaxation (Fig. 8.3), it becomes clear that the transfer of spectral weight from the central peak to the sidebands occurs in a time and frequency range that is below the uncertainty resolution limit, i.e., one has to know the spectrum $A(\omega, t)$ with a simultaneous accuracy which is better than $\Delta \omega \Delta t=2 \pi$ to resolve this process in detail. We thus conclude that the very rapid relaxation in the Hubbard model can hardly be distinguished from an instantaneous formation of the scattering background as in the Falicov-Kimball model within TRPES, unless sophisticated deconvolution schemes are used. Further discussion of this topic can be found in Ref. [73].

On the other hand, the time-resolved optical conductivity $\sigma\left(t, t^{\prime}\right)$ can be measured without uncertainty limitation. As an illustration, this is discussed for the Hubbard model in Figs. 8.4 and 8.3, for the same physical situation as in Fig. 8.2. Although the relation to the Green functions is not as direct as for TRPES, $\sigma(t, t-s)$ contains a lot

${ }^{5}$ Eq. 8.32 contains the lesser Green function, while the the retarded Green function is plotted in Fig. 8.2 However, the retarded function $G^{\mathrm{r}}$ is a linear combination of $G^{<}$(photoemission) and $G^{>}$(inverse photoemission) (table 2.2. The discussion would be very similar for lesser Green function [73, but the spectrum of the retarded Green function is more familiar from equilibrium many-body theory, and it is not cut off by the Fermi function. 


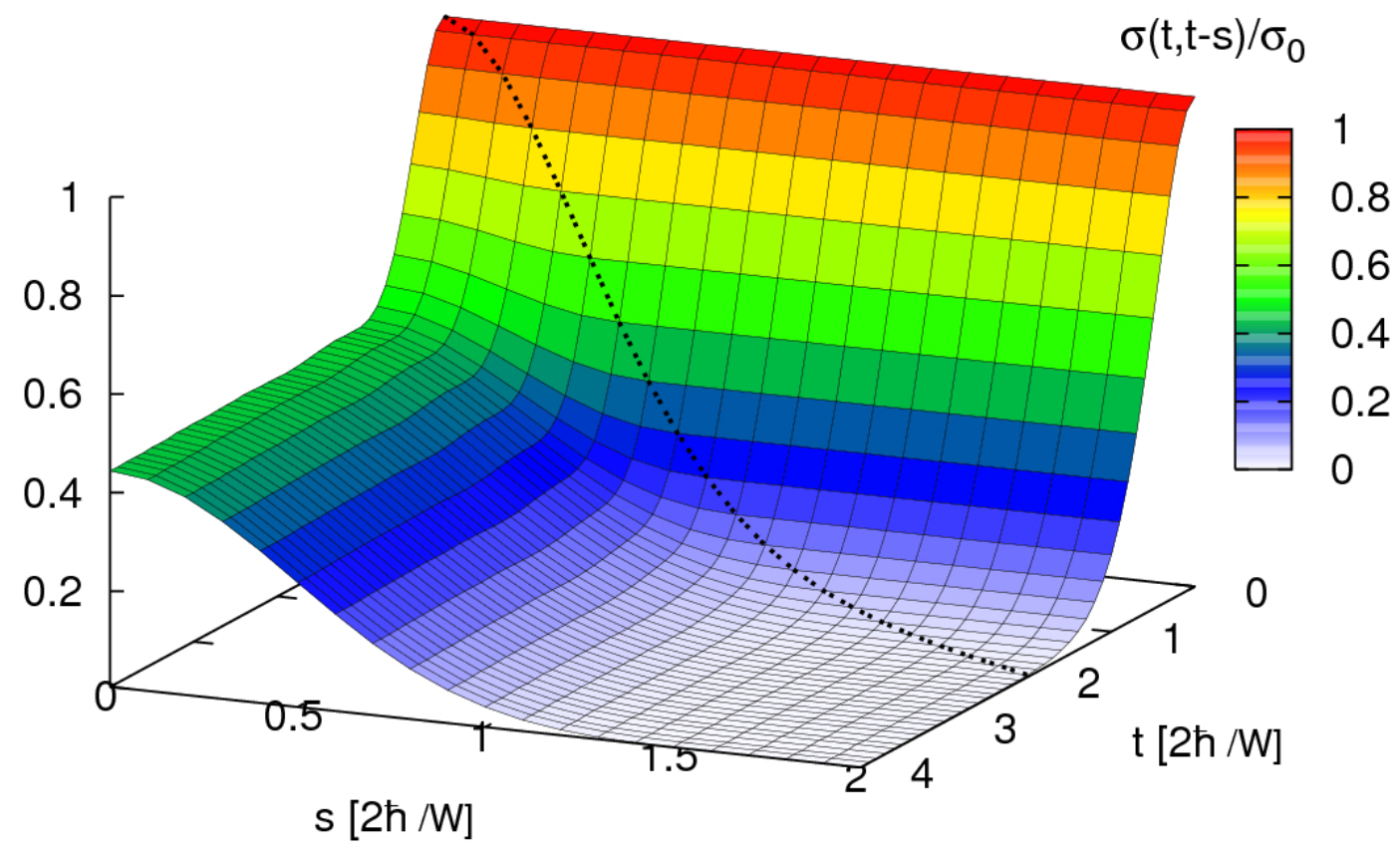

Figure 8.4: Optical conductivity $\sigma(t, t-s)$ for the quench from the noninteraction ground state in the Hubbard model (initial temperature $T=0$ ) to $U=3$. The unit of the conductivity is $\sigma_{0}=2 N a^{2} e^{2} V /\left(2 \hbar^{2} V\right)$, where $a$ is the lattice constant. The dashed line is at $t-s<0$.

of information on the state of the system at time $t$ after the excitation. For $t<0$ and $t \rightarrow \infty, \sigma(t, t-s)$ depends only on the time-difference $s$, indicating that the system is in a stationary state. For $t<0$ this is the initial equilibrium state, and for $t \rightarrow \infty$ it corresponds to the final steady state. In the initial state, $\sigma(t, t-s) \equiv \sigma(s)$ is constant as expected for the perfectly conducting noninteracting metal, while in the final state $\sigma(t, t-s)$ vanishes for $s \rightarrow \infty$. The latter implies that the Drude weight (8.13) vanishes in the final state, which is expected for the Hubbard model at finite interaction and temperature $T>0$. (The system is excited by the quench, and relaxes to a thermal state at finite temperature.) In the region $t-s<0$ (bounded by the dotted line in Fig. 8.4) $\sigma(t, t-s)$ determines the current after the pumping at $t=0$ caused by an electrical field applied to the sample before the pumping. It thus measures a combination of the electromagnetic response of the initial state and the subsequent decay of the induced current for $t>0$. The optical conductivity is constant as a function of $s$ in this regime because the initial state is a perfect conductor. By contrast, for $t-s>0, \sigma(t, t-s)$ describes the response of the nonequilibrium state alone, and hence gives direct insight into the relaxation after the perturbation. This relaxation turns out to be more or less 
monotonous, similar to the thermalization of the double occupancy and the momentum distribution in this parameter range (cf. Figs. 6.2 and 6.4). The relaxation becomes essentially complete already on timescales of a few times of the inverse bandwidth, after which the response is stationary and $\sigma(t, t-s)$ depends on $s$ only. Therefore the relaxation time and the time scales of the electromagnetic response, which is set by the decline of $\sigma(t, t-s)$ for $s \rightarrow \infty$, apparently have the same order of magnitude.

\subsubsection{Coherent electronic oscillations in time-resolved spectra Photoemission spectra}

We now compute spectroscopic signals of an excited Mott insulator, by means of the interaction quench from the metallic state to the strongly interacting regime $(U \gg V)$ in the Hubbard model and the Falicov-Kimball model. The metallic state at $t=0$ may thereby be viewed as an idealized way to describe a photodoped Mott insulator (Sec. 8.1). As in the previous section, Green functions are obtained from nonequilibrium DMFT, using a semielliptic density of states with quarter bandwidth $V$ (Ch. 5 and 6). It was discussed already in Ch. 6 (for the Hubbard model) and Sec. 5.3.4 (for the Falicov-Kimball model) that in the strong-coupling regime the relaxation is dominated by $2 \pi / U$-periodic collapse and revival oscillations. We will now demonstrate that these oscillations show up in a characteristic way in time-resolved photoemission and optical spectroscopy on an excited Mott insulator.

In Fig. 8.5 the angular-resolved photoemission spectrum $\mathcal{I}_{\boldsymbol{k}}\left(\omega ; t_{p}\right)$ is plotted at fixed momentum $\left[\epsilon_{\boldsymbol{k}}=1\right]$ after a quench in the Falicov-Kimball model from $U=1$ to $U=10$, using Gaussian pulses (8.30). Due to the energy uncertainty, all features of the spectrum except for its total weight, which is proportional to $n_{\boldsymbol{k}}(t)$, are washed out for short pulses $(\Delta t=0.2$, Fig. 8.5c). On the other hand, long pulses show only an average of the final and initial stationary state spectrum $(\Delta t=0.66$, Fig. 8.5 $)$. Only for intermediate pulses both the $2 \pi / U$-periodicity and the gap become visible $(\Delta t=0.33$, Fig. 8.5b).

Interestingly, the coherent oscillations are most pronounced in the center of the gap (Fig. 8.5b). This observation can be understood from the atomic limit of the Hamiltonian (1.1), i.e., for $V=0$. For the interaction term alone, $H_{U}=U \sum_{i} n_{i \uparrow} n_{i \downarrow}-\sum_{i \sigma} \mu_{\sigma} n_{i \sigma}$ the time evolution of annihilation operators is given by $e^{i H_{U} t} c_{j \sigma} e^{-i H_{U} t}=e^{i \mu_{\sigma} t}\left[c_{j \sigma}+\right.$ $\left.\left(e^{-i t U}-1\right) c_{j \sigma} n_{i \bar{\sigma}}\right]$. For $t, t^{\prime}>0$, the Green function then follows as

$$
G_{\boldsymbol{k} \sigma}^{<}\left(t, t^{\prime}\right)=i e^{i \mu_{\sigma}\left(t-t^{\prime}\right)}\left[A_{\boldsymbol{k} \sigma}+B_{\boldsymbol{k} \sigma} e^{i t^{\prime} U}+B_{\boldsymbol{k} \sigma}^{*} e^{-i t U}+C_{\boldsymbol{k} \sigma} e^{i U\left(t^{\prime}-t\right)}\right],
$$

with

$$
\begin{aligned}
A_{\boldsymbol{k} \sigma} & =\sum_{i j} e^{i \boldsymbol{k}\left(\boldsymbol{R}_{i}-\boldsymbol{R}_{j}\right)}\left\langle\left(1-n_{j \bar{\sigma}}\right) c_{j \sigma}^{\dagger} c_{i \sigma}\left(1-n_{i \bar{\sigma}}\right)\right\rangle_{0}, \\
B_{\boldsymbol{k} \sigma} & =\sum_{i j} e^{i \boldsymbol{k}\left(\boldsymbol{R}_{i}-\boldsymbol{R}_{j}\right)}\left\langle n_{j \bar{\sigma}} c_{j \sigma}^{\dagger} c_{i \sigma}\right\rangle_{0}, \\
C_{\boldsymbol{k} \sigma} & =\sum_{i j} e^{i \boldsymbol{k}\left(\boldsymbol{R}_{i}-\boldsymbol{R}_{j}\right)}\left\langle n_{j \bar{\sigma}} c_{j \sigma}^{\dagger} c_{i \sigma} n_{i \bar{\sigma}}\right\rangle_{0},
\end{aligned}
$$




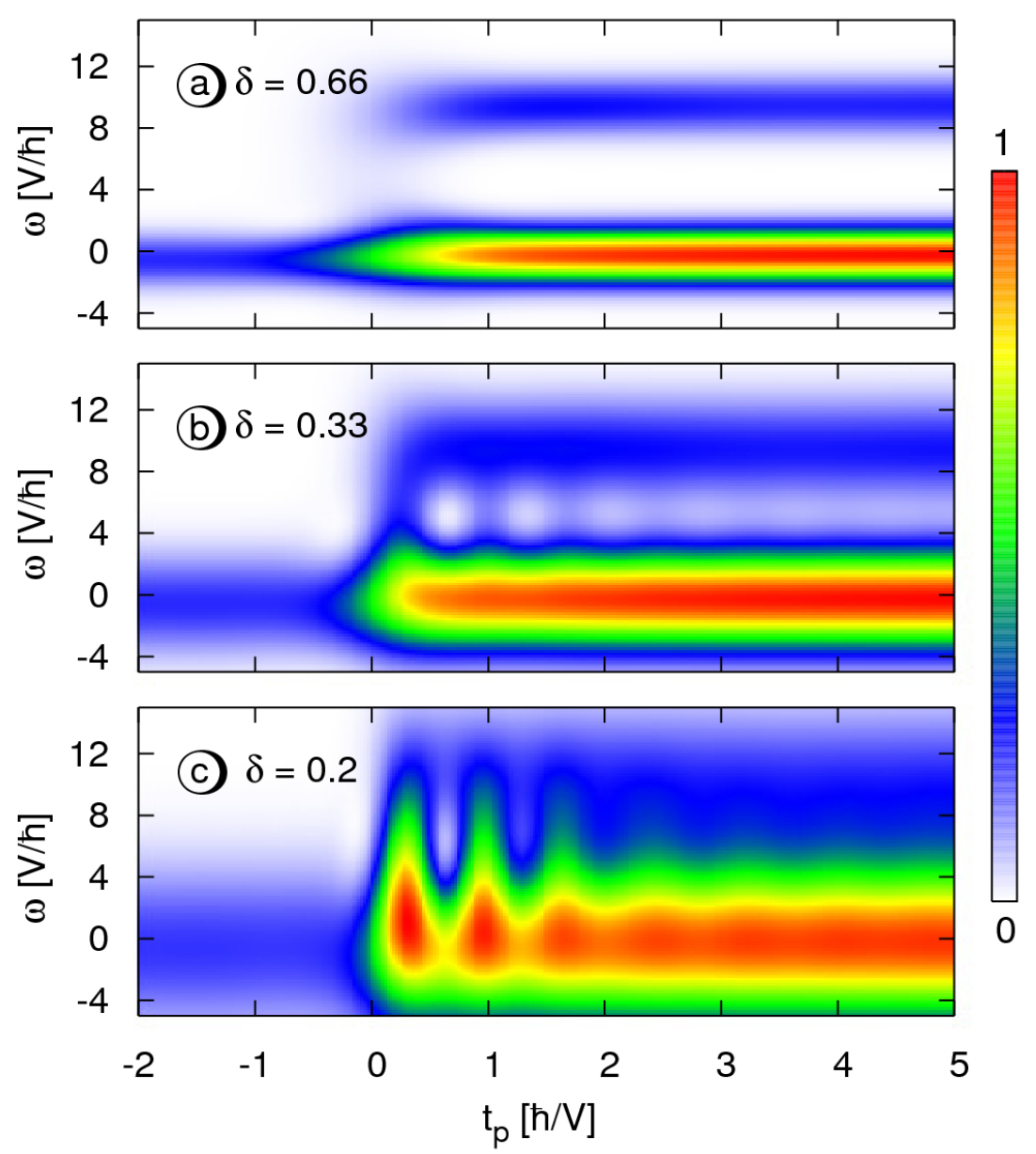

Figure 8.5: (Reprinted from Ref. 73]) Quench from $U=1$ to $U=10$ : Photoemission signal (a.u.) [Eq. (8.24)] for $\epsilon_{\boldsymbol{k}}=1$, and Gaussian probe envelopes 8.30) with $\Delta t=0.66$ (top), $\Delta t=0.33$ (middle), and $\Delta t=0.2$ (bottom). Pulse lengths $\Delta t$ are in units of $\hbar / V$ $=1$. 

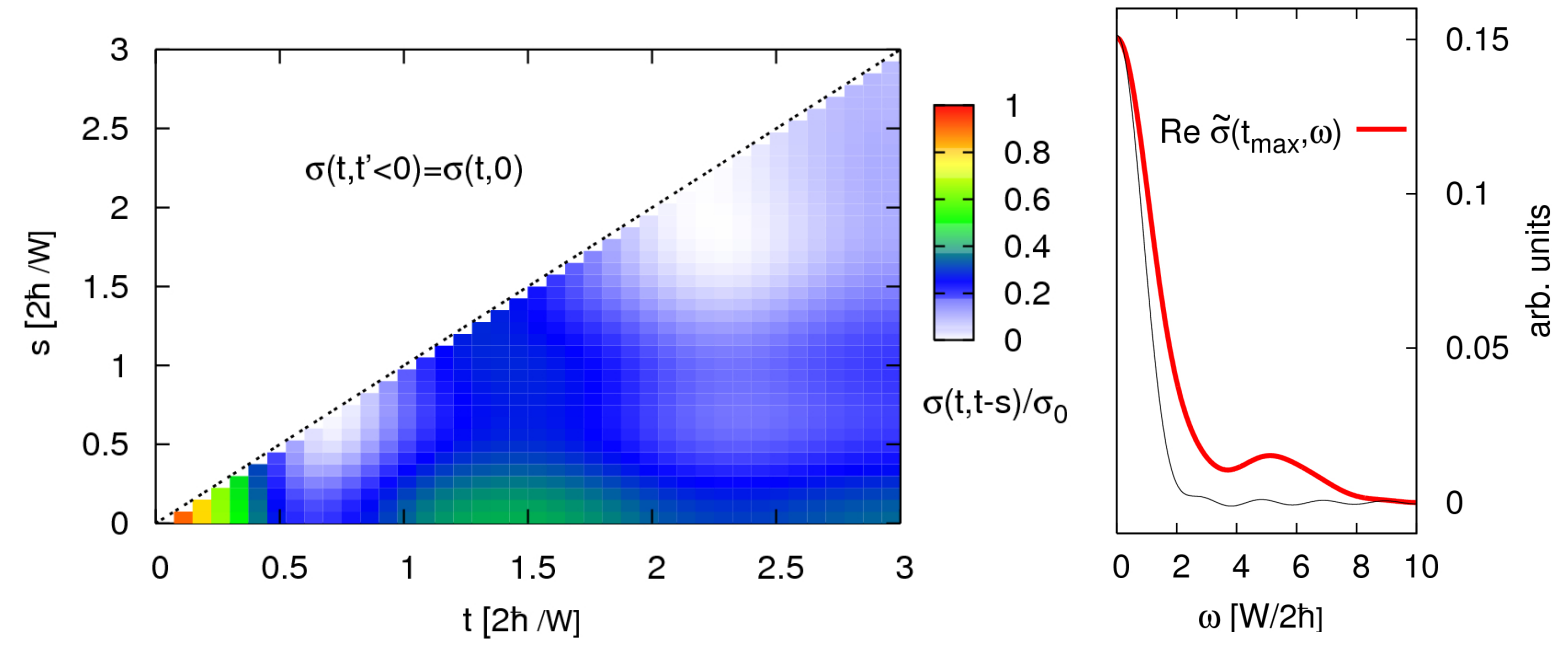

Figure 8.6: (Reprinted from Ref. [72]) Left: Optical conductivity $\sigma(t, t-s)$ for the quench from the noninteraction ground state in the Hubbard model (initial temperature $T=0$ ) to $U=5$. The unit of the conductivity is $\sigma_{0}=2 N a^{2} e^{2} V /\left(2 \hbar^{2} V\right)$, where $a$ is the lattice constant. In the region above the upper dashed line, $t-s<0$. Right: Fourier transform 8.14a of the optical conductivity at the largest time $t=3$. Since numerical state reach only up to $s=3$, we introduce a factor $\exp \left(-0.2 s^{2}\right)$ in the integral $8.14 \mathrm{a}$ ) to weaken the hard cutoff at $s=3$, which corresponds to a convolution $\sigma(t, \omega)$ with the kernel indicated by the this line.

and $\langle\cdot\rangle_{0}$ is the expectation value in the (arbitrary) state at $t=0$ immediately after the pump. Inserting this expression into Eq. (8.24) we find, for $t_{p} \gg \Delta t$,

$$
\begin{aligned}
\mathcal{I}_{\boldsymbol{k} \sigma}\left(\omega ; t_{p}\right) \propto A_{\boldsymbol{k} \sigma}\left|\tilde{S}\left(\omega+\mu_{\sigma}\right)\right|^{2}+C_{\boldsymbol{k} \sigma} \mid \tilde{S}(\omega & \left.+\mu_{\sigma}-U\right)\left.\right|^{2} \\
& +2 \operatorname{Re}\left[\tilde{S}(\omega) \tilde{S}\left(\omega+\mu_{\sigma}-U\right) B_{\boldsymbol{k} \sigma} e^{i t_{p} U}\right] .
\end{aligned}
$$

The first two terms are centered in the upper and lower Hubbard bands at $\omega=-\mu_{\sigma}$ and $\omega=U-\mu_{\sigma}$ and do not change with time. The third term, which oscillates with period $2 \pi / U$, has its maximum where $\tilde{S}\left(\omega+\mu_{\sigma}\right)$ and $\tilde{S}\left(\omega+\mu_{\sigma}-U\right)$ overlap. For Gaussian pulses, this is precisely the center of the gap because $\tilde{S}(\omega) \tilde{S}(\omega-U) \propto e^{-(\omega-U / 2)^{2} / 2 \Delta t^{2}} e^{-U^{2} / \Delta t^{2}}$. However, the central peak is suppressed by an exponentially factor $e^{-U^{2} / \Delta t^{2}}$, such that oscillations become invisible for pulses with duration $\Delta t \gg 1 / U$.

This discussion shows that on short time scales the observed time-dependent spectrum in the Falicov-Kimball model for large interactions resembles that of the atomic limit. The initial state at $t=0$ determines only the weight of the three components, but not the frequency of the oscillations. The oscillating midgap weight is thus not specific for one particular excitation process, but it is a universal property of the Mott insulator. Via this universal feature it may eventually become possible to observe collapse-and-revival oscillations in TRPES experiments on correlated materials. A more detailed analysis, 

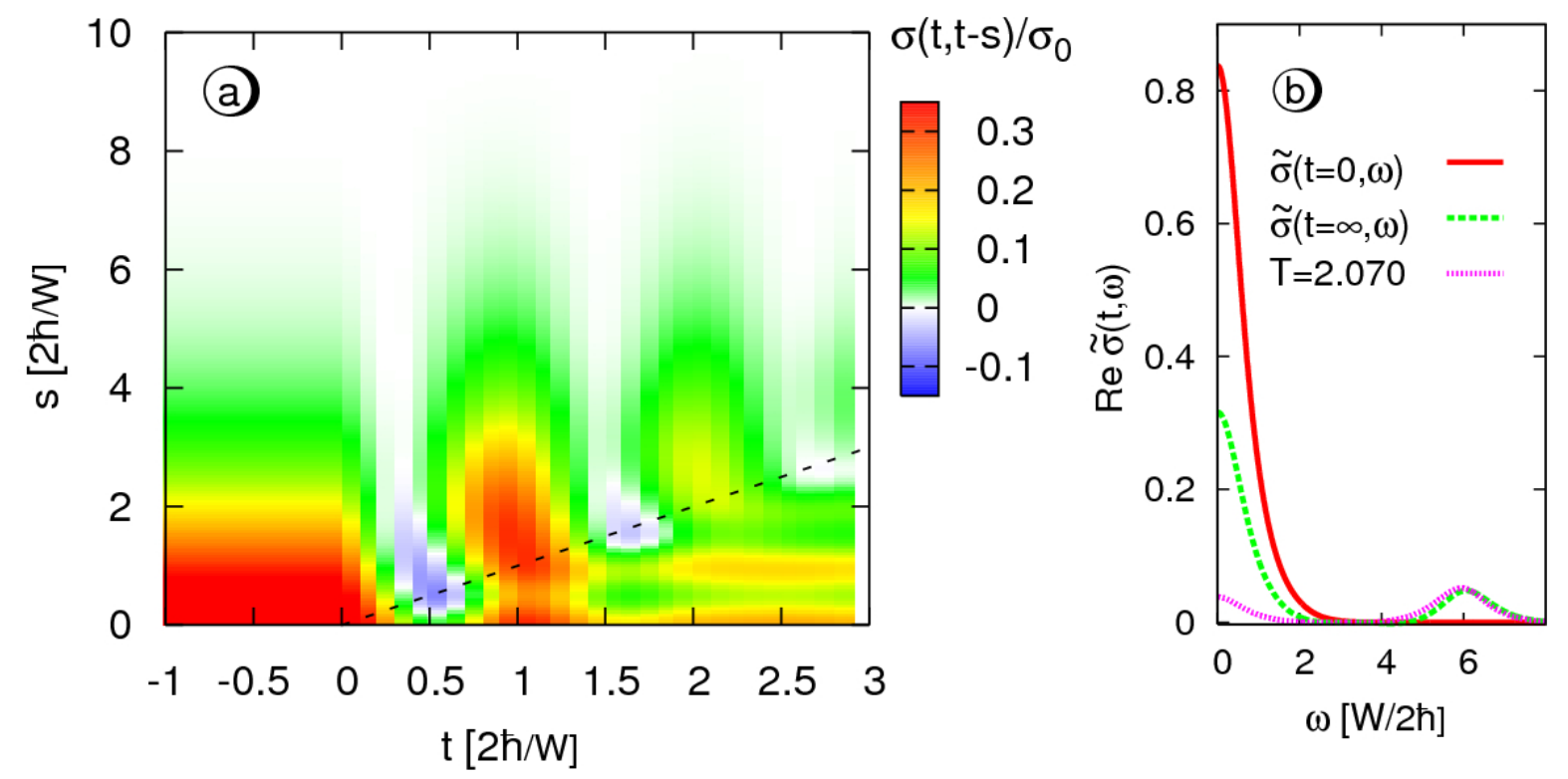

Figure 8.7: (a) Optical conductivity $\sigma(t, t-s)$ for the quench from the ground state at $U=1$ (initial temperature $T=0)$ to $U=6\left(n_{f}=n_{c}=1 / 2\right.$, half-bandwidth $\left.W=2\right)$ in the Falicov-Kimball model. The unit of the conductivity is $\sigma_{0}=N a^{2} e^{2} W /\left(2 \hbar^{2} V\right)$, where $a$ is the lattice constant. In the region above the upper dashed line, $t-s<0$. Below the lower dashed line the relaxation is essentially complete. (b) Fourier transform (8.14a) of the optical conductivity in the initial and final stationary state, and for an equilibrium state at $U=6$, with the same excitation energy relative as the final state $(T=2.070)$.

including also the damping of the oscillations, could be done within the strong-coupling approach of Sec. 4.4. As apparent from the general derivation, similar results as in Fig. 8.5 should hold also for the Hubbard model, but the times that can be accessed with CTQMC times in the strong-coupling regime (Ch. 6) are currently not sufficient to corroborate this proposition.

\section{Optical conductivity}

In the following we present the time-resolved optical conductivity $\sigma(t, t-s)$ as a function of $t$ and $s$ for the Hubbard model (Fig. 8.6), for a quench from the noninteracting state to $U=5$. From Ch. 6 it follows that collapse and revival oscillations in the double occupancy and the momentum occupation are visible for $U \gtrsim 3.3$. For $U=5$, the oscillations are already strongly damped, and the first maximum is slightly shifted from $2 \pi / U \approx 1.257$ to a larger value $t_{1} \approx 1.4$ (Fig. 6.4). The interpretation of the results in Fig. 8.6 very much resembles the discussion of Fig. 8.4 in the previous section: For $t<0$ and $t \rightarrow$ $\infty, \sigma(t, t-s)$ depends only on the time-difference $s$ and describes the electromagnetic 
response of the initial and final steady state, respectively. The Fourier transformation 8.14a of the conductivity at the largest time $t=3$ exhibits a peak at $\omega=0$ and a weak maximum at $\omega \approx U$ (Fig. 8.6 b). Although relaxation processes have not come to an end at the largest time, this indicates that the final state is indeed a correlated metal at very high temperature, with a broadened Drude peak and a charge excitation peak in the optical response. The electromagnetic response for both $t>0$ and $t-s>0$ is clearly dominated by oscillations, which are visible, e.g. along the line $s=0$ in Fig. 8.6 a, where the first maximum occurs at approximately same time $t_{1}$ as for the double occupancy and the momentum distribution.

Corresponding results for the Falicov-Kimball model are qualitatively, because the atomic limit is the same in both models (Fig. 8.7). The differences are that (i) the metallic state of the Falicov-Kimball model is never a perfect conductor because there is finite scattering of the electrons even at the Fermi surface, and (ii) the final state is not a thermal state. Due to (i), the Drude weight vanishes, and thus $\sigma(t, t-s) \rightarrow 0$ for $s \rightarrow \infty$ [cf. Eq. 8.13] ], and (ii) implies that the electromangentic response in the final state [which could be obtained directly by replacing the Fermi function in Eq. (8.16) with the effective Fermi function $F(\omega)$, Eq. 5.45 is between that of the initial metallic state and the thermally excited insulator (Fig. $8.7 \mathrm{~b}$ ) 


\section{Chapter 9}

\section{Conclusion and outlook}

In this thesis, nonequilibrium DMFT is further developed and applied to the time evolution of the Hubbard model and the Falicov-Kimball model after a quantum quench. The interaction quench in the Falicov-Kimball model is solved analytically (Ch. 5), thus providing an important benchmark for the application of DMFT to nonequilibrium situations. For the solution of the interaction quench in the Hubbard model, continuous-time quantum Monte Carlo is used as an impurity solver (Ch. 6). The solution of the two models in nonequilibrium reveals several interesting effects, such as the occurrence of nonthermal steady states after an interaction quench in the Falicov-Kimball model, and the very sensitive dependence of the relaxation behavior in the Hubbard model on the final interaction. The latter indicates the existence of a dynamical transition, which coincides with a regime of rapid thermalization. A general discussion of the dynamics of simple quantum mechanical systems is provided in Ch. 4, including the derivation of some criteria for the validity of gerealized Gibbs ensembles for the description of nonthermal steady states. The understanding of the dynamics in idealized models is a prerequisite for the understanding of more complicated many-particle systems, such as correlated materials after excitation with a laser pump-pulse. The description of such pump-probe experiments on correlated systems with nonequilibrium DMFT is explained in the last part of this thesis (Ch. 8).

What is the current status of nonequilibrium DMFT, and where is it going to develop in the future? Many technical issues have been resolved so far, and preliminary results such as the solution of the mapping problem in Sec. 3.4.4 show that further progress is within reach. In addition, some predictions for experiment can hopefully be tested soon using cold atomic gases (Ch. 6), and the framework is set up to apply DMFT within a realistic description of pump-probe experiments in correlated materials. Nonequilibrium DMFT is therefore most probably still in a rapidly developing state, and exciting advances can be expected in the near future. We thus conclude this thesis with a brief summary of open problems that may provide definite starting points for further investigations.

Two possible developments of nonequilibrium DMFT were already indicated in Ch. 3 . (i) Using the closed form self-consistency equations derived in Sec. 3.2 one can attempt to simplify the self-consistency for general density of states, as indicated at the end of Sec. 3.2. (ii) The solution of the mapping problem in Sec. 3.4 .4 should be brought into 
a form such that time-dependent DMRG becomes available as an impurity solver for nonequilibrium DMFT. This would most probably allow to study the Hubbard model at much larger times than those which are currently accessible within QMC, at least in the single-band case. However, also within QMC there is room for further improvements. Different implementations of the algorithm used in this thesis will allow to study interacting initial states instead of the noninteracting initial state that is assumed in Ch. 6, and different QMC algorithms may yield larger times. For example, a generalization of the hybridization expansion solver to the Keldysh contour is likely to be more efficient at strong-coupling that the weak-coupling method used at present.

The fundamental questions raised in Ch. 4 are rather difficult to answer in general, in particular the transition from ergodic to nonergodic behavior (Sec. 4.5). However, a good starting point is given by the investigation of prethermalization in almost integrable Hamiltonians, e.g., hard-core bosons with small hopping between next-nearest neighbors. In this context it would also be intersting to study the effect of a small but finite hopping amplitude of the $f$-particles in the Falicov-Kimball model, using unitary perturbation theory. Furthermore, it is still to be clarified whether the intermediate state in prethermalization is related the generalized Gibbs ensembles of the effective Hamiltonian.

The relaxation behavior in the Hubbard model leaves many questions to be answered. As already mentioned at the end of Ch. 6, the current analysis should be extended to more general initial states, to check whether a "dynamical critical point" is a general feature of the relaxation, and whether it is continuously related to the equilibrium phase transition. Using DMRG as an impurity solver, one might also be able to see whether a change in the long-time relaxation behavior occurs at the same critical interaction.

The excitation during slow ramps (Ch. 7) is closely related to the question of the optimal ramp between two parameters of the Hamiltonian, in which a system is least excited. Using CTQMC as impurity solver, this question can now be studied for the Hubbard model without major extensions of the method. For intermediate interactions, one can simulate the system up to two or three times the thermalization timescale in the quench, e.g., during a ramp-on of the hopping. The results can be compared to the perturbative analysis presented in Ch. 7, and the combination of both approaches may then give a robust description how to improve the experimental ramp procedures which are useed at present. This question is already accessible within the currently available methods and of immediate experimental interest. It should therefore be investigated in the near future.

Finally, the application of time-resolved spectroscopy in the field of strongly correlated systems leaves room for many investigations. The biggest challenges for theory are (i), the more realistic description of the pump-excitation system, and (ii), the interplay of the electronic system with slower degrees of freedom, such as localized spins or lattice vibrations. One system that is of experimental interest and could be investigated already with the currently available techniques is a charge transfer system, consisting of one correlated (d)-band and one uncorrelated (p)-band. If the pump-pulse excites electrons from the $\mathrm{p}$ into the into the $\mathrm{d}$-band, the subsequent relaxation will occur in two steps: On a very short timescale the electrons in the d-band will thermalize and form a doped 
Mott insulator or correlated metallic state. This process is followed by a much slower recombination, which has already been resolved in experiment in some systems. Shorter pulses might also be capable to resolve the formation of the doped Mott insulator in the near future.

The interplay of degrees of freedom on very different timescales could be studied in a setup where the slow degrees of freedom are treated classically, leading to classical equations of motion which are coupled to the electronic nonequilibrium correlation functions. When the slow degrees of freedom are given by coherent phonon modes, two questions are of possible interest: (i) How does the pump-excited electron system excite the lattice vibrations during its short relaxation? For this analysis one could possibly restrict the true electronic nonequilibrium calculation to very short times after the pump pulse, and use of the Born Oppenheimer approximation after this initial relaxation. (ii) In some cases, however the timescales of "slow" and "fast" degrees of freedom might mix up such that it is not possible to the Born-Oppenheimer approximation, e.g., when the electronic relaxation is delayed due to prethermalization. Then the problem becomes more difficult, because the electronic nonequilibrium motion must then be resolved on the timescale of the slow degrees of freedom. As a starting point, this could be investigated within the Falicov-Kimball model, where times of the order of 1000 in units of the inverse electronic bandwidth are numerically accessible (Ch. 7), or when the electronic problem is treated perturbatively (Sec. 3.4.3). However, when electronic interactions have to be treated in a nonperturbative way, new concepts are needed, such as a systematic expansion around a quantum kinetic equation. 


\section{Appendix A}

\section{Combination of CTQMC and DMFT}

\section{DMFT iteration}

In this Appendix we explain the computational scheme for the solution of the nonequilibrium DMFT equations for an interaction quench in the Hubbard model. The system is initially in the noninteracting state at temperature $T$ and half-filling, and the interaction is suddenly increased to a finite value $U$ at time $t=0$ [cf. Eq. (6.1)]. We assume a semielliptic density of states of the hopping matrix elements [Eq. (3.18)], such that the self-consistency equation is given by Eq. (3.19).

The DMFT iteration scheme is represented in Fig. A.1. Green functions $G_{\sigma}, G_{0, \sigma}$, and $\Lambda_{\sigma}$ satisfy the symmetry 2.15), such that they can be represented by their Matsubara, retarded, mixed " $\vdash$ ", and lesser component. The Matsubara Green functions are given by the equilibrium Green functions and represented by their Fourier transform

$$
g_{0, \sigma}^{\mathrm{M}}\left(i \omega_{n}\right)=\int d \epsilon \frac{\rho(\epsilon)}{i \omega_{n}+\mu-\epsilon}=g_{\sigma}^{\mathrm{M}}\left(i \omega_{n}\right)=V^{-2} \lambda_{\sigma}^{\mathrm{M}}\left(i \omega_{n}\right),
$$

where $\rho(\epsilon)$ is given by Eq. (3.18). In this expression, the first equality holds because the initial state is noninteracting, and the last equality holds due to the self-consistency (3.19. At half-filling, one has $\mu=0$. The mixed components $G_{\sigma}^{\ulcorner}, G_{0, \sigma}^{\ulcorner}$, and $\Lambda_{\sigma}^{-}$are represented by their value along the branch cut after the partial Fourier transform 2.20) and analytical continuation to complex frequencies, i.e., for each Green function $Y$ we keep two functions $Y^{\ulcorner}\left(\omega^{ \pm}, t\right)$ on a fixed frequency mesh. The latter is adapted to van Hove singularities at the band-edges.

The DMFT iteration is started from an initial guess for the local Green function $G_{\sigma}\left(t, t^{\prime}\right)$, for which we usually take the noninteracting equilibrium Green function,

$$
G_{\sigma}^{e q}\left(t, t^{\prime}\right)=i \int d \epsilon \rho(\epsilon) e^{i \epsilon\left(t^{\prime}-t\right)}\left[f(\epsilon)-\Theta_{C}\left(t, t^{\prime}\right)\right],
$$

where $\Theta_{C}\left(t, t^{\prime}\right)=1$ if $t$ is the contour theta function (Table 2.1). In step (i) [cf. Fig. A.1, the Weiss field $\Lambda\left(t, t^{\prime}\right)$ is computed from the closed self-consistency equation (3.19). This is then used to determine the noninteracting bath Green function $G_{0, \sigma}$ from its inverse (3.7b), as explained for Eq. 2.36 [step (ii) in Fig. A.1]. The function $G_{0, \sigma}$ is 


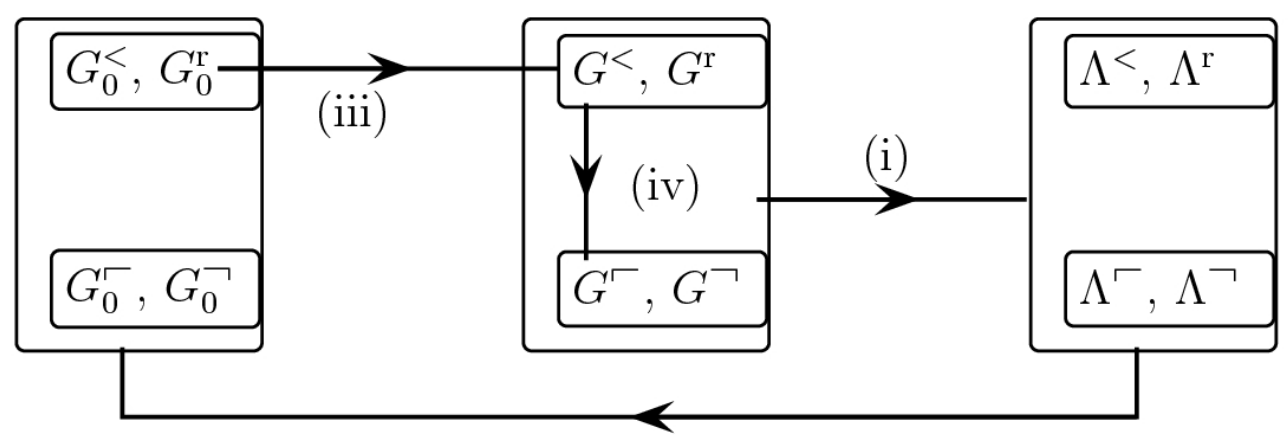

(ii)

Figure A.1: Computational scheme for the DMFT self-consistency iteration for the semielliptic density of states, using CTQMC (see text).

the input for the calculation of the interacting bath Green function (3.6) using CTQMC [step (iii) in Fig. A.1. Because the initial state is noninteracting, the Monte Carlo sampling can be restricted to the real-time branch of the contour. Then only the realtime components $G_{\sigma}^{\mathrm{r}}$ and $G_{\sigma}^{<}$are obtained, but the mixed component $G_{\sigma}^{\ulcorner}\left(\omega^{ \pm}, t\right)$ can be reconstructed from these functions and the previous Weiss field $\Lambda$ [step (iv)]: For this purpose consider the Dyson equation (3.7), which has the form of Eq. (2.36) after the replacement $\Lambda=\Lambda_{\sigma}+\Sigma_{\sigma}, G=G_{\sigma}$, and $h(t)=\mu$. Hence $G_{\sigma}^{\ulcorner}(z, t)$ can be obtained from the integral (2.48), making the same replacements. The self-energy $\Sigma_{\sigma}^{-}$, which enters this expression, is yet undetermined. However, $\Sigma_{\sigma}\left(t, t^{\prime}\right)$ is proportional to the interaction strenghts $U(t)$ and $U\left(t^{\prime}\right)$. Thus we have $\Sigma_{\sigma}^{r}(\tau, t)=0$ for a noninteracting initial state with $U(-i \tau)=U(0)=0$, and $G\left\ulcorner\left(\omega^{ \pm}, t\right)\right.$ is given by

$$
G_{\sigma}^{\ulcorner}\left(\omega^{ \pm}, t\right)=g_{\sigma}^{\mathrm{M}}\left(\omega^{ \pm}\right)\left[G_{\sigma}^{\mathrm{a}}(0, t)+\int_{0}^{t} d \bar{t} \Lambda_{\sigma}^{\ulcorner}\left(\omega^{ \pm}, \bar{t}\right) G_{\sigma}^{\mathrm{a}}(\bar{t}, t)\right]
$$

where $g_{\sigma}^{\mathrm{M}}\left(\omega^{ \pm}\right)=\mp \pi i \rho(\omega)$ [Eq. (A.1)]. Steps (i) through (iv) are repeated until convergence, which is reached after not more that 15 iterations for the results presented in this thesis.

\section{Determination of the self-energy}

After convergence of the DMFT equations, the self-energy $\Sigma_{\sigma}$ has to be calculated in order to obtain expectation values of various observables (Sec. 3.3). A straingtforward way to do this would be to compute the difference $\Sigma_{\sigma}\left(t, t^{\prime}\right)=G_{\sigma}^{-1}\left(t, t^{\prime}\right)-G_{0, \sigma}^{-1}\left(t, t^{\prime}\right)$ after time-discretization. However, this approach requires a subtle cancellation of (singular) derivative terms in $G_{\sigma}^{-1}\left(t, t^{\prime}\right)$ and $G_{0, \sigma}\left(t, t^{\prime}\right)$ at $t=t^{\prime}$ [cf. Eq. (3.7b] ], and is thus not suitable in praxis.

A second possibility to obtain $\Sigma_{\sigma}$ is the solution of the linear equation

$$
\Gamma_{\sigma}=\Sigma_{\sigma} * G_{\sigma},
$$


where $\Gamma_{\sigma}$ is the two-particle correlation function (3.9). The latter can in principle be measured using QMC. Eq. (A.4) has the form of Eq. (2.54), with $m=0$ and the replacement $Q=\Gamma_{\sigma}, G=\Sigma_{\sigma}$, and $\Lambda=G_{\sigma}$. Its solution therefore requires the solution of Volterra equations of the first kind, which can become numerically unstable in some cases (Sec. 2.5.2).

We therefore propose a third way to compute the self-energy. It is based on the a correlation function $X_{\sigma}$, which is defined by the Dyson-like equation $G_{\sigma}=G_{0, \sigma}+$ $G_{0, \sigma} * X_{\sigma} * G_{0, \sigma}$, and can be directly measured in the CTQMC algorithm. 17 By comparison of the the Dyson equation (3.7) in integral form, $G_{\sigma}=G_{0, \sigma}+G_{0, \sigma} * \Sigma_{\sigma} * G_{\sigma}$, with the definition of $X_{\sigma}, G_{\sigma}=G_{0, \sigma}+G_{0, \sigma} * X_{\sigma} * G_{0, \sigma}$, we find

$$
\Sigma_{\sigma} *\left(1+G_{0, \sigma} * X_{\sigma}\right)=X_{\sigma} .
$$

This equation now has the form of Eq. 2.54), with $m=1$, and it thus corresponds Volterra equations of the second kind, whose solution is generally better conditioned than the solution of Volerra equations of the first kind. The solution has been explained in Sec. 2.5.2,

\footnotetext{
${ }^{1}$ In fact, $X_{\sigma}$, and not $G_{\sigma}$, is the basic quantity that is sampled in the CTQMC algorithm. This modification of the original algortithm of Ref. [104] was introduced by $\mathrm{Ph}$. Werner to reduce the signproblem at half-filling, where only even orders of the expansion contribute to $X_{\sigma}$ (to be published).
} 


\section{Bibliography}

[1] J. Hubbard, Electron Correlations in Narrow Energy Bands, Proc. R. Soc. London Ser. A 276, 238 (1963).

[2] M. C. Gutzwiller, Effect of Correlation on the Ferromagnetism of Transition Metals, Phys. Rev. Lett. 10, 159 (1963).

[3] J. Kanamori, Electron Correlation and Ferromagnetism of Transition Metals, Prog. theo. Phys. 30, 275 (1963).

[4] E. H. Lieb and F. Y. Wu, Absence of Mott Transition in an Exact Solution of the Short-Range, One-Band Model in One Dimension, Phys. Rev. Lett. 20, 1445 (1968).

[5] P. W. Anderson, Theory of magnetic exchange interactions - exchange in insulators and semiconductors, Solid State Physics 14, 99 (1963).

[6] Y. Nagaoka, Ferromagnetism in a narrow almost half-filled s band, Phys. Rev. 147, 392 (1966).

[7] N. Mott, Metal-insulator transitions, Taylor and Francis, London, 1990.

[8] M. Imada, A. Fujimori, and Y. Tokura, Metal-insulator transitions, Rev. Mod. Phys. 70, 1039 (1998).

[9] J. Hubbard, Electron correlations in narrow energy bands. II. degenerate band case, Proc. Roy. Soc. A 277, 237 (1964).

[10] W. F. Brinkman and T. M. Rice, Application of Gutzwiller's Variational Method to the Metal-Insulator Transition, Phys. Rev. B 2, 4302 (1970).

[11] A. Georges, G. Kotliar, W. Krauth, and M. J. Rozenberg, Dynamical mean-field theory of strongly correlated fermion systems and the limit of infinite dimensions, Rev. Mod. Phys. 68, 13 (1996).

[12] I. Bloch, J. Dalibard, and W. Zwerger, Many-body physics with ultracold gases, Rev. Mod. Phys. 80, 885 (2008).

[13] M. Greiner, O. Mandel, T. Esslinger, T. W. Hänsch, and I. Bloch, Nature 415, 39 (2002).

[14] R. Jördens, N. Strohmaier, K. Günter, H. Moritz, and T. Esslinger, A Mott insulator of fermionic atoms in an optical lattice, Nature 455, 204 (2008).

[15] U. Schneider, L. Hackermüller, S. Will, T. Best, I. Bloch, T. A. Costi, R. W. Helmes, D. Rasch, and A. Rosch, Metallic and Insulating Phases of Repulsively Interacting Fermions in a 3D Optical Lattice, Science 322, 1520 (2008).

[16] M. Greiner, O. Mandel, T. W. Hänsch, and I. Bloch, Nature 419, 51 (2002). 
[17] B. S. Shastry and B. Sutherland, Twisted boundary conditions and effective mass in Heisenberg-Ising and Hubbard rings, Phys. Rev. Lett. 65, 243 (1990).

[18] D. J. Scalapino, S. R. White, and S. C. Zhang, Superfluid density and the Drude weight of the Hubbard model, Phys. Rev. Lett. 68, 2830 (1992).

[19] R. Peierls, Zur Theorie des Diamagnetismus von Leitungselektronen, Z. Physik 80, 763 (1933).

[20] J. M. Luttinger, The Effect of a Magnetic Field on Electrons in a Periodic Potential, Phys. Rev. 84, 814 (1951).

[21] W. Kohn, Theory of Bloch Electrons in a Magnetic Field: The Effective Hamiltonian, Phys. Rev. 115, 1460 (1959).

[22] B. A. Foreman, Consequences of local gauge symmetry in empirical tight-binding theory, Phys. Rev. B 66, 165212 (2002).

[23] H. Haug and A.-P. Jauho, Quantum Kinetics in Transport and Optics of Semiconductors, Springer, Berlin, 2nd edition, 2008.

[24] R. van Leeuwen, N. E. Dahlen, G. Stefanucci, C.-O. Almbladh, and U. von Barth, Introduction to the Keldysh formalism and applications to time-dependent densityfunctional theory, arXiv:cond-mat/0506130.

[25] L. P. Kadanoff and G. Baym, Quantum Statistical Mechanics, Benjamin, New York, 1962.

[26] G. D. Mahan, Electron transport in solids, Phys. Rep. 110, 321 (1984).

[27] J. Rammer and H. Smith, Quantum field-theoretical methods in transport theory of metals, Rev. Mod. Phys. 58, 323 (1986).

[28] L. V. Keldysh, Diagram Technique for Nonequilibrium Processes, J. Exptl. Theoret. Phys. 47, 1515. [Sov. Phys. JETP, 20,1018 (1965)] (1964).

[29] J. Schwinger, Brownian Motion of a Quantum Oscillator, J. Math. Phys. 2, 407-432 (1961).

[30] S. Fujita, Thermodynamic Evolution Equation for a Quantum Statistical Gas, J. of Math. Phys. 6, 1877 (1965).

[31] R. A. Craig, Perturbation Expansion for Real-Time Green's Functions, J. of Math. Phys. 9, 605 (1968).

[32] D. Kremp, M. Schlanges, and T. Bornath, Nonequilibrium Real-time Greenfunctions and the Condition of Weakening of Initial Correlation, J. of Stat. Phys. 41, 661 (1985).

[33] P. Danielewicz, Quantum theory of nonequilibrium processes, I, Ann. Physics 152, 239 (1984).

[34] M. Wagner, Expansions of nonequilibrium Green's functions, Phys. Rev. B 44, 6104 (1991).

[35] D. Semkat, D. Kremp, and M. Bonitz, Kadanoff-Baym equations with initial correlations, Phys. Rev. E 59, 1557 (1999).

[36] M. Bonitz and D. Semkat (editors), Progress in Nonequilibrium Green's Functions II, World Scientific, Singapore, 2003.

[37] J. W. Negele and H. Orland, Quantum Many-Particle Systems, Addison-Wesley, Redwood City, 1988. 
[38] J. Berges, Introduction to Nonequilibrium Quantum Field Theory, arXiv:hep-ph/0409233.

[39] G. Baym and L. P. Kadanoff, Conservation Laws and Correlation Functions, Phys. Rev. 124, 287 (1961).

[40] G. Baym, Self-Consistent Approximations in Many-Body Systems, Phys. Rev. 127, 1391 (1962).

[41] J. M. Luttinger and J. C. Ward, Ground-State Energy of a Many-Fermion System. II, Phys. Rev. 118, 1417 (1960).

[42] M. Potthoff, Non-perturbative construction of the Luttinger-Ward functional, Condens. Matt. Phys. 9, 557 (2006).

[43] J. K. Freericks, V. M. Turkowski, and V. Zlatić, Nonequilibrium Dynamical MeanField Theory, Phys. Rev. Lett. 97, 266408 (2006).

[44] J. K. Freericks, Quenching Bloch oscillations in a strongly correlated material: Nonequilibrium dynamical mean-field theory, Phys. Rev. B 77, 075109 (2008).

[45] N. E. Dahlen and R. van Leeuwen, Solving the Kadanoff-Baym Equations for Inhomogeneous Systems: Application to Atoms and Molecules, Phys. Rev. Lett. 98, 153004 (2007).

[46] H. Brunner and P. J. van der Houwen, The numerical solution of Volterra equations, North-Holland, Amsterdam, 1986.

[47] U. Brandt and C. Mielsch, Thermodynamics and correlation functions of the Falicov-Kimball model in large dimensions, Z. Phys. B: Condens. Matter 75, 365 (1989).

[48] A. Georges and G. Kotliar, Hubbard model in infinite dimensions, Phys. Rev. B 45, 6479 (1992).

[49] W. Metzner and D. Vollhardt, Correlated Lattice Fermions in $\mathrm{d}=\infty$ Dimensions, Phys. Rev. Lett. 62, 324 (1989).

[50] T. Maier, M. Jarrell, T. Pruschke, and M. H. Hettler, Quantum cluster theories, Rev. Mod. Phys. 77, 1027 (2005).

[51] M. Potthoff, M. Aichhorn, and C. Dahnken, Variational Cluster Approach to Correlated Electron Systems in Low Dimensions, Phys. Rev. Lett. 91, 206402 (2003).

[52] G. Kotliar and D. Vollhardt, Strongly Correlated Materials: Insights from Dynamical Mean-Field Theory, Phys. Today 57 (3), 53 (2004).

[53] K. Held, I. A. Nekrasov, G. Keller, V. Eyert, N. Blümer, A. K. McMahan, R. T. Scalettar, T. Pruschke, V. I. Anisimov, and D. Vollhardt, Realistic investigations of correlated electron systems with LDA + DMFT, phys. stat. sol. (b) 243, 2599 (2006).

[54] M. Potthoff and W. Nolting, Surface metal-insulator transition in the Hubbard model, Phys. Rev. B 59, 2549 (1999).

[55] K. Byczuk and D. Vollhardt, Correlated bosons on a lattice: Dynamical mean-field theory for Bose-Einstein condensed and normal phases, Phys. Rev. B 77, 235106 (2008).

[56] E. Müller-Hartmann, The Hubbard-model at high dimensions - some exact results and weak coupling theory, Z. Phys. B 76, 211 (1989). 
[57] E. Müller-Hartmann, Correlated fermions on lattice in high dimensions, Z. Phys. B 74, 507 (1989).

[58] B. Menge and E. Müller-Hartmann, The Hubbard-model at high dimensions - selfconsistent weak-coupling theory, Z. Phys. B 82, 237 (1991).

[59] H. Schweitzer and G. Czycholl, Weak-coupling treatment of the Hubbard-model in one-dimension, 2-dimension and 3-dimension, Z. Phys. B 83, 93 (1991).

[60] R. Vlaming and D. Vollhardt, Controlled mean-field theory for disordered electronic systems: Single-particle properties, Phys. Rev. B 45, 4637 (1992).

[61] D. Vollhardt, Investigation of correlated electron systems using the limit of high dimension, in "Correlated Electron Systems", ed. V.J. Emery, p. 57, World Scientific, Singapore, 1993.

[62] P. Schmidt and H. Monien, Nonequilibrium dynamical mean-field theory of a strongly correlated system, arXiv:cond-mat/0202046.

[63] V. Turkowski and J. K. Freericks, Nonlinear response of Bloch electrons in infinite dimensions, Phys. Rev. B 71, 085104 (2005).

[64] V. Turkowski and J. K. Freericks, Nonequilibrium perturbation theory of the spinless Falicov-Kimball model: Second-order truncated expansion in U, Phys. Rev. B 75, 125110 (2007).

[65] J. K. Freericks and V. M. Turkowski, Steady-state nonequilibrium dynamical meanfield theory and the quantum Boltzmann equation, J. Phys.: Confer. Ser. 35, 39 (2006).

[66] V. Turkowski and J. K. Freericks, Nonequilibrium sum rules for the retarded selfenergy of strongly correlated electrons, Phys. Rev. B 77, 205102 (2008).

[67] J. K. Freericks, V. M. Turkowski, and V. Zlatić, f-electron spectral function of the Falicov-Kimball model in infinite dimensions: The half-filled case, Phys. Rev. B 71, 115111 (2005).

[68] N. Tsuji, T. Oka, and H. Aoki, Correlated electron systems periodically driven out of equilibrium: Floquet + DMFT formalism, Phys. Rev. B 78, 235124 (2008).

[69] A. V. Joura, J. K. Freericks, and T. Pruschke, Steady-State Nonequilibrium Density of States of Driven Strongly Correlated Lattice Models in Infinite Dimensions, Phys. Rev. Lett. 101, 196401 (2008).

[70] N. Tsuji, T. Oka, and H. Aoki, Nonequilibrium steady state in photoexcited correlated electrons with dissipation, arXiv:0903.2332.

[71] M. Eckstein and M. Kollar, Nonthermal steady states after an interaction quench in the Falicov-Kimball model, Phys. Rev. Lett. 100, 120404 (2008).

[72] M. Eckstein and M. Kollar, Theory of time-resolved optical spectroscopy on correlated electron systems, Phys. Rev. B 78, 205119 (2008).

[73] M. Eckstein and M. Kollar, Measuring correlated electron dynamics with timeresolved photoemission spectroscopy, Phys. Rev. B 78, 245113 (2008).

[74] M. Eckstein, M. Kollar, and P. Werner, Thermalization after an Interaction Quench in the Hubbard Model, Phys. Rev. Lett. 103, 056403 (2009).

[75] E. N. Economou, Green's Functions in Quantum Physics, Springer-Verlag, Berlin, 1979. 
[76] G. D. Mahan, Energy bands of the Bethe lattice, Phys. Rev. B 63, 155110 (2001).

[77] M. Eckstein, M. Kollar, K. Byczuk, and D. Vollhardt, Hopping on the Bethe lattice: Exact results for densities of states and dynamical mean-field theory, Phys. Rev. B 71, 235119 (2005).

[78] M. Kollar, M. Eckstein, K. Byczuk, N. Blm̈er, P. van Dongen, M. H. R. de Cuba, W. Metzner, D. Tanasković, V. Dobrosavljević, G. Kotliar, and D. Vollhardt, Green functions for nearest- and next-nearest-neighbor hopping on the Bethe lattice, Ann. Phys. 14 (2005).

[79] N. Blümer and P. G. J. van Dongen, Transport properties of correlated electrons in high dimensions, in Concepts in Electron Correlation, edited by A. C. Hewson and V. Zlatić, NATO Science Series, Kluwer, 2003.

[80] A. Weisse, G. Wellein, A. Alvermann, and H. Fehske, The kernel polynomial method, Rev. Mod. Phys. 78, 275 (2006).

[81] M. Caffarel and W. Krauth, Exact diagonalization approach to correlated fermions in infinite dimensions: Mott transition and superconductivity, Phys. Rev. Lett. 72, 1545 (1994).

[82] R. Bulla, Zero Temperature Metal-Insulator Transition in the Infinite-Dimensional Hubbard Model, Phys. Rev. Lett. 83, 136 (1999).

[83] R. Bulla, T. A. Costi, and D. Vollhardt, Finite-temperature numerical renormalization group study of the Mott transition, Phys. Rev. B 64, 045103 (2001).

[84] R. Bulla, T. A. Costi, and T. Pruschke, Numerical renormalization group method for quantum impurity systems, Rev. of Mod. Phys. 80, 395 (2008).

[85] D. J. García, K. Hallberg, and M. J. Rozenberg, Dynamical Mean Field Theory with the Density Matrix Renormalization Group, Phys. Rev. Lett. 93, 246403 (2004).

[86] U. Schollwöck, The density-matrix renormalization group, Rev. Mod. Phys. 77, 259 (2005).

[87] F. B. Anders and A. Schiller, Real-Time Dynamics in Quantum-Impurity Systems: A Time-Dependent Numerical Renormalization-Group Approach, Phys. Rev. Lett. 95, 196801 (2005).

[88] A. J. Daley, C. Kollath, U. Schollwöck, and G. Vidal, Time-dependent densitymatrix renormalization-group using adaptive effective Hilbert spaces, J. Stat. Mech.: Theor. Exp., P04005 (2004).

[89] S. R. White and A. E. Feiguin, Real-Time Evolution Using the Density Matrix Renormalization Group, Phys. Rev. Lett. 93, 076401 (2004).

[90] X. Y. Zhang, M. J. Rozenberg, and G. Kotliar, Mott transition in the $d=\infty$ Hubbard model at zero temperature, Phys. Rev. Lett. 70, 1666 (1993).

[91] M. Jarrell, Hubbard model in infinite dimensions: A quantum Monte Carlo study, Phys. Rev. Lett. 69, 168 (1992).

[92] M. J. Rozenberg, X. Y. Zhang, and G. Kotliar, Mott-Hubbard transition in infinite dimensions, Phys. Rev. Lett. 69, 1236 (1992).

[93] A. Georges and W. Krauth, Numerical solution of the $d=\infty$ Hubbard model: Evidence for a Mott transition, Phys. Rev. Lett. 69, 1240 (1992). 
[94] M. Jarrell and J. E. Gubernatis, Bayesian inference and the analytic continuation of imaginary-time quantum Monte Carlo data, Phys. Rep. 269, 133 (1996).

[95] J. E. Hirsch and R. M. Fye, Monte Carlo Method for Magnetic Impurities in Metals, Phys. Rev. Lett. 56, 2521 (1986).

[96] A. N. Rubtsov, V. V. Savkin, and A. I. Lichtenstein, Continuous-time quantum Monte Carlo method for fermions, Phys. Rev. B 72, 035122 (2005).

[97] E. Gull, P. Werner, O. Parcollet, and M. Troyer, Continuous-time auxiliary-field Monte Carlo for quantum impurity models, Europhy. Lett. 82, 57003 (2008).

[98] P. Werner, A. Comanac, L. de' Medici, M. Troyer, and A. J. Millis, ContinuousTime Solver for Quantum Impurity Models, Phys. Rev. Lett. 97, 076405 (2006).

[99] P. Werner and A. J. Millis, Hybridization expansion impurity solver: General formulation and application to Kondo lattice and two-orbital models, Phys. Rev. B 74, 155107 (2006).

[100] P. Werner and A. J. Millis, Doping-driven Mott transition in the one-band Hubbard model, Phys. Rev. B 75, 085108 (2007).

[101] E. Gull, P. Werner, A. Millis, and M. Troyer, Performance analysis of continuoustime solvers for quantum impurity models, Phys. Rev. B 76, 235123 (2007).

[102] P. Werner, E. Gull, and A. J. Millis, Metal-insulator phase diagram and orbital selectivity in three-orbital models with rotationally invariant Hund coupling, Phys. Rev. B 79, 115119 (2009).

[103] L. Mühlbacher and E. Rabani, Real-Time Path Integral Approach to Nonequilibrium Many-Body Quantum Systems, Phys. Rev. Lett. 100, 176403 (2008).

[104] P. Werner, T. Oka, and A. J. Millis, Diagrammatic Monte Carlo simulation of nonequilibrium systems, Phys. Rev. B 79, 035320 (2009).

[105] M. Schiro and M. Fabrizio, Real-Time Diagrammatic Monte Carlo for Nonequilibrium Quantum Transport, arXiv:0808.0589.

[106] M. Toda, R. Kubo, and N. Saitô, Statistical physics I, Springer, Berlin, 1992.

[107] J. R. Dorfman, An Introduction to Chaos in Nonequilibrium Statistical Mechanics, Cambridge University Press, Cambridge, 1999.

[108] M. C. Gutzwiller, Chaos in Classical and Quantum Mechanics, Springer Verlag, New-York, 1990.

[109] E. T. Jaynes, Information Theory and Statistical Mechanics, Phys. Rev. 106, 620 (1957).

[110] N. Krylov, Works on Foundations of Statistical Physics, Princeton Univ. Press, Princeton, 1979.

[111] D. Snász, Ergodicity of classical billard balls, Physica A 194, 86 (1993).

[112] E. Fermi, J. Pasta, S. Ulam, and M. Tsingou, Studies of nonlinear problems I, Los Alamos preprint LA-1940 (1955).

[113] J. Ford, The Fermi-Pasta-Ulam problem: Paradox turns discovery, Phys. Rep. 213 (1992).

[114] Focus Issue: The Fermi-Pasta-Ulam problem - The first 50 years, Chaos 15, 015101 (2005). 
[115] N. J. Zabusky and M. D. Kruskal, Interaction of solitons in a collisionless plasma and the recurrence of initial states, Phys. Rev. Lett 15, 240 (1965).

[116] B. Chirikov, Resonance processes in magnetic traps, J. Nuclear Energy C 1, 253 (1960).

[117] M. Pettini, L. Casetti, M. Cerruti-Sola, R. Franzosi, and C. E. G. D., Weak and strong Chaos in Fermi-Pasta-Ulam models and beyond, Chaos 15, 015106 (2005).

[118] M. Cramer, C. M. Dawson, J. Eisert, and T. J. Osborne, Exact Relaxation in a Class of Nonequilibrium Quantum Lattice Systems, Phys. Rev. Lett. 100, 030602 (2008).

[119] S. P. Heims, Approach to equilibrium, Am. J. Phys. 33, 722 (1965).

[120] T. Kinoshita, T. Wenger, and D. S. Weiss, A quantum Newton's cradle, Nature 440, 900 (2006).

[121] S. Hofferberth, I. Lesanovsky, B. Fischer, T. Schumm, and J. Schmiedmayer, Nonequilibrium coherence dynamics in one-dimensional Bose gases, Nature 449, 324 (2007).

[122] M. Rigol, A. Muramatsu, and M. Olshanii, Hard-core bosons on optical superlattices: Dynamics and relaxation in the superfluid and insulating regimes, Phys. Rev. A 74, 053616 (2006).

[123] M. Rigol, V. Dunjko, V. Yurovsky, and M. Olshanii, Relaxation in a completely integrable many-body quantum system: An ab initio study of the dynamics of the highly excited states of 1D lattice hard-core bosons, Phys. Rev. Lett. 98, 050405 (2007).

[124] C. Kollath, A. M. Läuchli, and E. Altman, Quench Dynamics and Nonequilibrium Phase Diagram of the Bose-Hubbard Model, Phys. Rev. Lett. 98, 180601 (2007).

[125] G. Roux, Quenches in quantum many-body systems: One-dimensional BoseHubbard model reexamined, Phys. Rev. A 79, 021608 (2009).

[126] M. Kollar and M. Eckstein, Relaxation of a one-dimensional Mott insulator after an interaction quench, Phys. Rev. A 78, 013626 (2008).

[127] M. Möckel and S. Kehrein, Interaction Quench in the Hubbard Model, Phys. Rev. Lett. 100, 175702 (2008).

[128] M. Möckel and S. Kehrein, Real-time evolution for weak interaction quenches in quantum systems, arXiv:0903.1561.

[129] M. Rigol, V. Dunjko, and M. Olshanii, Thermalization and its mechanism for generic isolated quantum systems, Nature 452, 854 (2008).

[130] M. Rigol, Breakdown of thermalization in finite one-dimensional systems, arXiv:0904.3746.

[131] M. A. Cazalilla, Effect of Suddenly Turning on Interactions in the Luttinger Model, Phys. Rev. Lett. 97, 156403 (2006).

[132] A. Iucci and M. A. Cazalilla, Quantum quench dynamics of some exactly solvable models in one dimension, arXiv:0903.1205.

[133] S. R. Manmana, S. Wessel, R. M. Noack, and A. Muramatsu, Strongly Correlated Fermions after a Quantum Quench, Phys. Rev. Lett. 98, 210405 (2007). 
[134] S. R. Manmana, S. Wessel, R. M. Noack, and A. Muramatsu, Time evolution of correlations in strongly interacting fermions after a quantum quench, Phys. Rev. B 79, 155104 (2009).

[135] P. Barmettler, M. Punk, V. Gritsev, E. Demler, and E. Altman, Relaxation of Antiferromagnetic Order in Spin-1/2 Chains Following a Quantum Quench, Phys. Rev. Lett. 102, 130603 (2009).

[136] J. von Neumann, Beweis des Ergodensatzes und des H-Theorems in der neuen Mechanik, Z. Phys. A 57, 30 (1929).

[137] J. M. Deutsch, Quantum statistical-mechanics in a closed system, Phys. Rev. A 43, 2046 (1991).

[138] M. Srednicki, Chaos and quantum thermalization, Phys. Rev. E 50, 888 (1994).

[139] P. Reimann, Typicality for Generalized Microcanonical Ensemble, Phys. Rev. Lett. 99, 160404 (2007).

[140] P. Bocchieri and A. Loinger, Ergodic Foundation of Quantum Statistical Mechanics, Phys. Rev. 114, 948 (1959).

[141] S. Goldstein, J. L. Lebowitz, R. Tumulka, and N. Zanghí, Canonical typicality, Phys. Rev. Lett. 96, 050403 (2006).

[142] M. V. Berry, Regular and irregular semiclassical wavefunctions, J. Phys. A 10, 2083 (1977).

[143] M. D. Girardeau, Decay of magnetization in the one-dimensional XY model, Phys. Lett. A 30, 442 (1969).

[144] E. Barouch and M. Dresden, Exact Time-Dependent Analysis for the OneDimensional XY Model, Phys. Rev. Lett. 23, 114 (1969).

[145] E. Barouch, B. M. McCoy, and M. Dresden, Statistical Mechanics of the XY Model. I, Phys. Rev. A 2, 1075 (1970).

[146] M. D. Girardeau, Relaxation of localized magnetization in the one-dimensional XY model, Phys. Lett. A 32, 67 (1970).

[147] E. Barouch and B. M. McCoy, Statistical Mechanics of the XY Model. II. SpinCorrelation Functions, Phys. Rev. A 3, 786 (1971).

[148] F. Gebhard and A. E. Ruckenstein, Exact results for a Hubbard chain with longrange hopping, Phys. Rev. Lett. 68, 244 (1992).

[149] T. Barthel and U. Schollwöck, Dephasing and the Steady State in Quantum ManyParticle Systems, Phys. Rev. Lett. 100, 100601 (2008).

[150] B. Sutherland, Beautiful models, World Scientific, Singapore, 2004.

[151] D. C. Brody, D. W. Hook, and L. P. Hughston, Unitarity, ergodicity and quantum thermodynamics, J. Phys. A: Math. Theor. 40, F503 (2007).

[152] D. M. Gangardt and M. Pustilnik, Correlations in an expanding gas of hard-core bosons, Phys. Rev. A 77, 041604(R) (2008).

[153] J. Berges, S. Borsányi, and C. Wetterich, Prethermalization, Phys. Rev. Lett. 93, 142002 (2004).

[154] G. Aarts, G. F. Bonini, and C. Wetterich, Exact and truncated dynamics in nonequilibrium field theory, Phys. Rev. D 63, 025012 (2000). 
[155] A. B. Harris and R. V. Lange, Single-Particle Excitations in Narrow Energy Bands, Phys. Rev. 157, 295 (1967).

[156] A. H. MacDonald, S. M. Girvin, and D. Yoshioka, $t / U$ expansion for the Hubbard model, Phys. Rev. B 37, 9753 (1988).

[157] A. Rosch, D. Rasch, B. Binz, and M. Vojta, Metastable Superfluidity of Repulsive Fermionic Atoms in Optical Lattices, Phys. Rev. Lett. 101, 265301 (2008).

[158] M. Henon and C. Heiles, Applicability of the Third Integral Of Motion - Some Numerical Experiments, Astronomical Journal 69, 73 (1964).

[159] W. Götze and L. Sjögren, Relaxation processes in supercooled liquids, Rep. Prog. Phys. 55, 241 (1992).

[160] A. C. Cassidy, D. Mason, V. Dunjko, and M. Olshanii, Threshold for Chaos and Thermalization in the One-Dimensional Mean-Field Bose-Hubbard Model, Phys. Rev. Lett. 102, 025302 (2009).

[161] L. M. Falicov and J. C. Kimball, Simple Model for Semiconductor-Metal Transitions: $\mathrm{SmB}_{6}$ and Transition-Metal Oxides, Phys. Rev. Lett. 22, 997 (1969).

[162] R. Ramirez, L. M. Falicov, and J. C. Kimball, Metal-insulator transitions: A simple theoretical model, Phys. Rev. B 2, 3383 (1970).

[163] R. Ramirez and L. M. Falicov, Theory of the $\alpha-\gamma$ Phase Transition in Metallic Cerium, Phys. Rev. B 3, 2425 (1970).

[164] U. Brandt and R. Schmidt, Exact results for the distribution of thef-level ground state occupation in the spinless Falicov-Kimball model, Z. Phys. B: Condens. Matter 63, 45 (1986).

[165] U. Brandt and R. Schmidt, Ground state properties of a spinless Falicov-Kimball model; additional features, Z. Phys. B: Condens. Matter 67, 43 (1987).

[166] T. Kennedy and E. Lieb, An itinerant electron model with crystalline or magnetic long range order, Physica A 138, 320 (1986).

[167] C. Gruber and N. Macris, The Falicov-Kimball model: A review of exact results and extensions, Helv. Phys. Acta 69 (1996).

[168] U. Gavish and Y. Castin, Matter-Wave Localization in Disordered Cold Atom Lattices, Phys. Rev. Lett. 95, 020401 (2005).

[169] K. V. Krutitsky, M. Thorwart, R. Egger, and R. Graham, Ultracold bosons in lattices with binary disorder, Phys. Rev. A 77, 053609 (2008).

[170] P. G. J. van Dongen and D. Vollhardt, Exact mean-field Hamiltonian for fermionic lattice models in high dimensions, Phys. Rev. Lett. 65, 1663 (1990).

[171] J. K. Freericks and V. Zlatić, Exact dynamical mean-field theory of the FalicovKimball model, Rev. Mod. Phys. 75, 1333 (2003).

[172] U. Brandt and C. Mielsch, Thermodynamics of the Falicov-Kimball model in large dimensions II, Z. Phys. B: Condens. Matter 79, 295 (1990).

[173] U. Brandt and C. Mielsch, Free energy of the Falicov-Kimball model in large dimensions, Z. Phys. B: Condens. Matter 82, 37 (1991).

[174] P. G. J. van Dongen, Exact mean-field theory of the extended simplified Hubbard model, Phys. Rev. B 45, 2267 (1992). 
[175] P. G. J. van Dongen and C. Leinung, Mott-Hubbard transition in a magnetic field, Annalen der Physik 509, 45 (1997).

[176] R. J. Elliott, J. A. Krumhansl, and P. L. Leath, The theory and properties of randomly disordered crystals and related physical systems, Rev. Mod. Phys. 46, 465 (1974).

[177] J. K. Freericks, Dynamical mean-field theory for strongly correlated inhomogeneous multilayered nanostructures, Phys. Rev. B 70, 195342 (2004).

[178] M.-T. Tran, Initial correlations in a nonequilibrium Falicov-Kimball model, Phys. Rev. B 78, 125103 (2008).

[179] M. Ulmke, V. Janiš, and D. Vollhardt, Anderson-Hubbard model in infinite dimensions, Phys. Rev. B 51, 10411 (1995).

[180] N. Blümer, Mott-Hubbard Metal-Insulator Transition and Optical Conductivity in High Dimensions (PhD thesis), Shaker Verlag, Aachen, 2003.

[181] M. Köhl, H. Moritz, T. Stöferle, K. Günter, and T. Esslinger, Fermionic Atoms in a Three Dimensional Optical Lattice: Observing Fermi Surfaces, Dynamics, and Interactions, Phys. Rev. Lett. 94, 080403 (2005).

[182] L. D. Landau, Zur Theorie der Energieubertragung. II, Phys. Z. Sowjetunion 2, 46 (1932).

[183] C. Zener, Non-Adiabatic Crossing of Energy Levels, Proc. R. Soc. Lond. A 137, 696 (1932).

[184] M. Wubs, K. Saito, S. Kohler, P. Hänggi, and Y. Kayanuma, Gauging a Quantum Heat Bath with Dissipative Landau-Zener Transitions, Phys. Rev. Lett. 97, 200404 (2006).

[185] A. Polkovnikov and V. Gritsev, Breakdown of the adiabatic limit in low-dimensional gapless systems, Nat. Phys. 4, 477 (2008).

[186] B. Damski and W. H. Zurek, Adiabatic-impulse approximation for avoided level crossings: From phase-transition dynamics to Landau-Zener evolutions and back again, Phys. Rev. A 73, 063405 (2006).

[187] A. Polkovnikov, Universal adiabatic dynamics in the vicinity of a quantum critical point, Phys. Rev. B 72, 161201 (2005).

[188] W. H. Zurek, U. Dorner, and P. Zoller, Dynamics of a Quantum Phase Transition, Phys. Rev. Lett. 95, 105701 (2005).

[189] J. Dziarmaga, Dynamics of a Quantum Phase Transition: Exact Solution of the Quantum Ising Model, Phys. Rev. Lett. 95, 245701 (2005).

[190] F. M. Cucchietti, B. Damski, J. Dziarmaga, and W. H. Zurek, Dynamics of the Bose-Hubbard model: Transition from a Mott insulator to a superfluid, Phys. Rev. A 75, 023603 (2007).

[191] J. Dziarmaga, Dynamics of a quantum phase transition in the random Ising model: Logarithmic dependence of the defect density on the transition rate, Phys. Rev. B 74, 064416 (2006).

[192] A. Altland and V. Gurarie, Many Body Generalization of the Landau-Zener Problem, Phys. Rev. Lett. 100, 063602 (2008). 
[193] R. Barankov and L. S. Levitov, Dynamical selection in developing fermionic pairing, Phys. Rev. A 73, 033614 (2006).

[194] R. Barankov and A. Polkovnikov, Optimal Nonlinear Passage Through a Quantum Critical Point, Phys. Rev. Lett. 101, 076801 (2008).

[195] S. Mondal, K. Sengupta, and D. Sen, Theory of defect production in nonlinear quench across a quantum critical point, Phys. Rev. B 79, 045128 (2009).

[196] A. H. Zewail, Femtochemistry: Atomic-Scale Dynamics of the Chemical Bond, J. Phys. Chem. A 104, 5660 (2000).

[197] V. M. Axt and T. Kuhn, Femtosecond spectroscopy in semiconductors: a key to coherences, correlations and quantum kinetics, Rep. Prog. Phys. 67, 433 (2004).

[198] H. Petek and S. Ogawa, Femtosecond time-resolved two-photon photoemission studies of electron dynamics in metals, Prog. Surf. Sci. 56, 239 (1997).

[199] T. Ogasawara, M. Ashida, N. Motoyama, H. Eisaki, S. Uchida, Y. Tokura, H. Ghosh, A. Shukla, S. Mazumdar, and M. Kuwata-Gonokami, Ultrafast Optical Nonlinearity in the Quasi-One-Dimensional Mott Insulator $\mathrm{Sr}_{2} \mathrm{CuO}_{3}$, Phys. Rev. Lett. 85, 2204 (2000).

[200] S. Iwai, M. Ono, A. Maeda, H. Matsuzaki, H. Kishida, H. Okamoto, and Y. Tokura, Ultrafast Optical Switching to a Metallic State by Photoinduced Mott Transition in a Halogen-Bridged Nickel-Chain Compound, Phys. Rev. Lett. 91, 057401 (2003).

[201] M. Chollet, L. Guerin, N. Uchida, S. Fukaya, H. Shimoda, T. Ishikawa, K. Matsuda, T. Hasegawa, A. Ota, H. Yamochi, G. Saito, R. Tazaki, S. Adachi, and S. Koshihara, Gigantic Photoresponse in 1/4-Filled-Band Organic Salt (EDO-TTF) ${ }_{2} \mathrm{PF}_{6}$, Science 307, 86 (2005).

[202] H. Okamoto, K. Ikegami, T. Wakabayashi, Y. Ishige, J. Togo, H. Kishida, and H. Matsuzaki, Ultrafast Photoinduced Melting of a Spin-Peierls Phase in an Organic Charge-Transfer Compound, K-Tetracyanoquinodimethane, Phys. Rev. Lett. 96, 037405 (2006).

[203] H. Okamoto, H. Matsuzaki, T. Wakabayashi, Y. Takahashi, and T. Hasegawa, Photoinduced Metallic State Mediated by Spin-Charge Separation in a One-Dimensional Organic Mott Insulator, Phys. Rev. Lett. 98, 037401 (2007).

[204] L. Perfetti, P. A. Loukakos, M. Lisowski, U. Bovensiepen, H. Berger, S. Biermann, P. S. Cornaglia, A. Georges, and M. Wolf, Time Evolution of the Electronic Structure of 1T-TaS 2 through the Insulator-Metal Transition, Phys. Rev. Lett. 97, 067402 (2006).

[205] L. Perfetti, P. A. Loukakos, M. Lisowski, U. Bovensiepen, M. Wolf, H. Berger, S. Biermann, and A. Georges, Femtosecond dynamics of electronic states in the Mott insulator 1T-TaS2 by time resolved photoelectron spectroscopy, New J. of Phys. 10, 053019 (2008).

[206] C. Kübler, H. Ehrke, R. Huber, R. Lopez, A. Halabica, R. F. Haglund, and A. Leitenstorfer, Coherent Structural Dynamics and Electronic Correlations during an Ultrafast Insulator-to-Metal Phase Transition in $\mathrm{VO}_{2}$, Phys. Rev. Lett. 99, 116401 (2007). 
[207] D. Polli, M. Rini, S. Wall, R. W. Schönlein, Y. Tomioka, Y. Tokura, G. Cerullo, and A. Cavalleri, Coherent orbital waves in the photo-induced insulator-metal dynamics of a magnetoresistive manganite, Nature Materials 6, 643 (2007).

[208] D. A. Mazurenko, A. A. Nugroho, T. T. M. Palstra, and P. H. M. van Loosdrecht, Dynamics of Spin and Orbital Phase Transitions in $\mathrm{YVO}_{3}$, Phys. Rev. Lett. 101, 245702 (2008).

[209] G. Steinmeyer, D. Sutter, L. Gallmann, N. Matuschek, and U. Keller, Frontiers in Ultrashort Pulse Generation: Pushing the Limits in Linear and Nonlinear Optics, Science 286, 1507 (1999).

[210] M. Hentschel, R. Kienberger, C. Spielmann, G. A. Reider, N. Milosevic, T. Brabec, P. Corkum, U. Heinzmann, M. Drescher, and F. Krausz, Attosecond metrology, Nature 414, 509 (2001).

[211] A. L. Cavalieri, N. Mueller, T. Uphues, V. S. Yakovlev, A. Baltuska, B. Horvath, B. Schmidt, L. Bluemel, R. Holzwarth, S. Hendel, M. Drescher, U. Kleineberg, P. M. Echenique, R. Kienberger, F. Krausz, and U. Heinzmann, Attosecond spectroscopy in condensed matter, Nature 449, 1029 (2007).

[212] FLASH (The Free-Electron Laser in Hamburg), http://hasyweb.desy.de/flash_booklet/flash_brochure.pdf.

[213] A. Cavalleri, C. Tóth, C. W. Siders, J. A. Squier, F. Ráksi, P. Forget, and J. C. Kieffer, Femtosecond Structural Dynamics in $\mathrm{VO}_{2}$ during an Ultrafast Solid-Solid Phase Transition, Phys. Rev. Lett. 87, 237401 (2001).

[214] A. Cavalleri, M. Rini, H. H. W. Chong, S. Fourmaux, T. E. Glover, P. A. Heimann, J. C. Kieffer, and R. W. Schönlein, Band-Selective Measurements of Electron Dynamics in $\mathrm{VO} 2$ Using Femtosecond Near-Edge X-Ray Absorption, Phys. Rev. Lett. 95, 067405 (2005).

[215] A. Rousse, C. Rischel, S. Fourmaux, I. Uschmann, S. Sebban, G. Grillon, P. Balcou, E. Förster, J. P. Geindre, P. Audebert, J. C. Gauthier, and D. Hulin, Non-thermal melting in semiconductors measured at femtosecond resolution, Nature 410, 65 (2001).

[216] M. Rini, R. Tobey, N. Dean, J. Itatani, Y. Tomioka, Y. Tokura, R. W. Schönlein, and A. Cavalleri, Control of the electronic phase of a manganite by mode-selective vibrational excitation, Nature 449, 72 (2007).

[217] P. B. Allen, Theory of thermal relaxation of electrons in metals, Phys. Rev. Lett. 59, 1460 (1987).

[218] J. K. Freericks, H. R. Krishnamurthy, Y. Ge, A. Y. Liu, and T. Pruschke, Theoretical description of time-resolved pump/probe photoemission in TaS_2: a single-band DFT+DMFT(NRG) study within the quasiequilibrium approximation, arXiv:0809.2347.

[219] W. S. Fann, R. Storz, H. W. K. Tom, and J. Bokor, Direct measurement of nonequilibrium electron-energy distributions in subpicosecond laser-heated gold films, Phys. Rev. Lett. 68, 2834 (1992). 
[220] R. Huber, F. Tauser, A. Brodschelm, M. Bichler, G. Abstreiter, and A. Leitenstorfer, How many-particle interactions develop after ultrafast excitation of an electron-hole plasma, Nature 414, 286 (2001).

[221] R. Huber, F. Tauser, A. Brodschelm, M. Bichler, G. Abstreiter, and A. Leitenstorfer, Femtosecond buildup of Coulomb screening in photoexcited GaAs probed via ultrabroadband THz spectroscopy, J. Luminesc. 94, 555 (2001).

[222] I. Leonov, N. Binggeli, D. Korotin, V. I. Anisimov, N. Stojic, and D. Vollhardt, Structural Relaxation due to Electronic Correlations in the Paramagnetic Insulator $\mathrm{KCuF}_{3}$, Phys. Rev. Lett. 101, 096405 (2008).

[223] L. Bányai, Q. T. Vu, B. Mieck, and H. Haug, Ultrafast Quantum Kinetics of Time-Dependent RPA-Screened Coulomb Scattering, Phys. Rev. Lett. 81, 882-885 (1998).

[224] N.-H. Kwong and M. Bonitz, Real-Time Kadanoff-Baym Approach to Plasma Oscillations in a Correlated Electron Gas, Phys. Rev. Lett. 84, 1768 (2000).

[225] J. T. Kindt and C. A. Schmuttenmaer, Theory for determination of the lowfrequency time-dependent response function in liquids using time-resolved terahertz pulse spectroscopy, J. Chem. Phys. 110, 8589 (1999).

[226] M. C. Beard and C. A. Schmuttenmaer, Using the finite-difference time-domain pulse propagation method to simulate time-resolved $\mathrm{THz}$ experiments, J. Chem. Phys. 114, 2903 (2001).

[227] J. M. Schins, E. Hendry, M. Bonn, and H. G. Müller, Retrieving the susceptibility from time-resolved terahertz experiments, J. Chem. Phys. 127, 094308 (2007).

[228] T. Pruschke, D. L. Cox, and M. Jarrell, Hubbard model at infinite dimensions: Thermodynamic and transport properties, Phys. Rev. B 47, 3553 (1993).

[229] A. Khurana, Electrical conductivity in the infinite-dimensional Hubbard model, Phys. Rev. Lett. 64, 1990 (1990).

[230] J. K. Freericks, H. R. Krishnamurthy, and T. Pruschke, Theoretical Description of Time-Resolved Photoemission Spectroscopy: Application to Pump-Probe Experiments, Phys. Rev. Lett. 102, 136401 (2009).

[231] M. Sakaue, T. Munakata, H. Kasai, and A. Okiji, Nonequilibrium Green functions depending on the observation time for ultrafast dynamics, Phys. Rev. B 66, 094302 (2002).

[232] L. Hedin and J. Lee, Sudden approximation in photoemission and beyond, J. of Elec. Spec. and Rel. Phen. 124, 289 (2002).

[233] C. Caroli, D. Lederer-Rozenblatt, B. Roulet, and D. Saint-James, Inelastic Effects in Photoemission: Microscopic Formulation and Qualitative Discussion, Phys. Rev. B 8, 4552 (1973).

[234] W. Schattke and M. A. van Hove, Solid-State Photoemission and Related Methods, Wiley-VCH, Weinheim, 2003.

[235] F. Reinert and S. Hüfner, Photoemission spectroscopy - from early days to recent applications, New J. Phys. 7, 97 (2005). 


\section{Danksagung}

Mein Dank gilt ...

... Prof. Dr. Dieter Vollhardt für ein äusserst interessantes Dissertationsthema in einem sehr aktiven Forschungsgebiet, für zahlreiche Diskussionen und wertvolle Hinweise zur Arbeit. Prof. Vollhardt hat mir zudem die Teilnahme an diversen Konferenzen sowie zwei Sommerschulen ermöglicht (in Greifswald und Trieste), und er hat auch sonst alles getan damit ich mich möglichst unbehelligt von anderweitigen Pflichten meiner Dissertation widmen konnte.

... Dr. Marcus Kollar für die unzähligen Stunden, die er in den letzten Jahren für Diskussionen aufgebracht hat, sowie für genaues Korrekturlesen des vorliegenden Manuscripts.

... Prof. Dr. Philipp Werner von der ETH in Zürich für spannende Zusammenarbeit im letzten Jahr meiner Dissertation, sowie für die zweimalige Einladung nach Zürich.

... allen anderen Mitgliedern und ehemaligen Mitgliedern des Lehrstuhls für zahlreiche anregende Gespräche, nicht nur über Physik.

... unseren Sekretärinnen Frau Besslich und Frau Seidl für kompetente Hilfe bei allen verwaltungstechnischen Fragen.

... Prof. Dr. Thomas Pruschke und Prof. Dr. Stephan Kehrein für Einladungen nach Göttingen sowie an die LMU in München, für die freundliche Betreuung vor Ort und für interessante Gespräche.

... zahlreichen noch nicht genannten Wissenschaftlern, die als Gast eine Zeit an unserem Lehrstuhl verbracht haben, für wertvolle Diskussionen zum Thema der Dissertation: Prof. Dr. Hans Kroha, Dr. Marcos Rigol, Dr. Corinna Kollath, Prof. Dr. Jim Freericks, Robert Peters, Michael Möckel, Matthias Punk, Dr. Salvatore Manmana, Dr. Takashi Oka, und Naoto Tsuji.

... der Studienstiftung des Deutschen Volkes für finanzielle Unterstützung sowie für die Teilnahme am Doktorandenforum in Borken-Gemen, das mir als motivierendes Erlebnis in Erinnerung bleiben wird, und meinem meinen Vetrauensdozenten Prof. Dr. Kiffman für die gute Betreuung der Augsburger Stipendiatengruppe.

... meinen Eltern für stete Unterstützung während des Studiums.

... Melanie für sehr viel Geduld und moralische Unterstützung. 


\section{List of publications}

Parts of this thesis have been published in the following references:

1) M. Eckstein and M. Kollar, Nonthermal steady states after an interaction quench in the Falicov-Kimball model, Phys. Rev. Lett. 100, 120404 (2008).

2) M. Kollar and M. Eckstein, Relaxation of a one-dimensional Mott insulator after an interaction quench, Phys. Rev. A 78, 013626 (2008).

3) M. Eckstein and M. Kollar, Theory of time-resolved optical spectroscopy on correlated electron systems, Phys. Rev. B 78, 205119 (2008).

4) M. Eckstein and M. Kollar, Measuring correlated electron dynamics with timeresolved photoemission spectroscopy, Phys. Rev. B 78, 245113 (2008).

5) M. Eckstein, M. Kollar, and P. Werner, Thermalization after an Interaction Quench in the Hubbard Model, Phys. Rev. Lett. 103, 056403 (2009).

Some parts of this thesis, which contain rather long and detailed calculations, are taken from those papers without major modifications. These parts are listed below:

- The derivation of criteria for the validity of generalized Gibbs ensembles (Sec. 4.3 , pages 58, 60 can be found in reference 2) above.

- The discussion of the role of degeneracies in the description of the long-time limit (Sec. 4.3, page 61) can be found in reference 2) above.

- Details of the analytical solution of the interaction quench in the Falicov-Kimball model (Sec. 5.2, pages 75, 80 can be found in reference 4) above.

- The derivation of the nonequilibrium optical conductivity in DMFT (Sec. 8.2.2, pages 121, 123) can be found in reference 3) above.

- The calculation of the time-resolved photoemission spectrum in the atomic limit of the Hubbard model (Sec. 8.4.2, pages 134 136) can be found in reference 4) above. 


\section{Lebenslauf}
Name:
Martin Eckstein
Geburtsdatum: 4. Dezember 1979
Staatsangehörigkeit: deutsch

\section{Tabellarischer Werdegang:}

1986 bis 1988: Grundschule in Augsburg Hochzoll

1988 bis 1990: Gundschule in Friedberg/Bay.

1990 bis 1999: Wernher-von-Braun Gymnasium in Friedberg

1999:

Abitur

1999 bis 2000: Zivildienst am Krankenhaus Friedberg

Oktober 2000

- März 2006: Physikstudium an der Universität Augsburg

2005 bis 2006: Diplomarbeit zum Thema

The frustrated Hubbard model on the Bethe lattice -

an investigation using the self-energy functional approach

bei Prof. Dr. Dieter Vollhardt,

Lehrstuhl für Theoretische Physik III

Institut für Physik der Universität Augsburg

Mai 2006: Beginn der Dissertation am selben Lehrstuhl 